


\title{
Orientation and organization of the presynaptic active zone protein Bassoon: from the Golgi to the synapse
}

\author{
Dissertation
}

for the award of the degree

"Doctor rerum naturalium"

of the Georg-August-Universität Göttingen

within the doctoral program CNMPB-GGNB

of the Georg-August University School of Science (GAUSS)

submitted by

Tina Ghelani

Born in Kolkata, 02.01.1986

March $22^{\text {nd }} 2016$, Göttingen 


\section{Thesis Committee}

Dr. Nina Wittenmayer

(Deptartment of Anatomy and Embrology, University Medical Center Göttingen)

Prof. Dr. Blanche Schwappach.

(Department of Molecular Biology, University Medical Center Göttingen)

Prof. Dr. Fred S. Wouters

(Laboratory for Molecular and Cellular Systems, Institute for Neuropathology, University Medical Center Göttingen)

\section{Members of the Examination Board}

$\underline{1^{\text {st }}}$ Referee: Prof. Dr. Thomas Dresbach

(Deptartment of Anatomy and Embrology, University Medical Center Göttingen)

$\underline{2^{\text {nd }}}$ Referee: Prof. Dr. Blanche Schwappach

(Department of Molecular Biology, University Medical Center Göttingen)

\section{Further members of the Examination Board}

Prof. Dr. Fred Wouters

(Laboratory for Molecular and Cellular Systems, Institute for Neuropathology, University Medical Center Göttingen)

Dr. Nina Wittenmayer

(Deptartment of Anatomy and Embrology, University Medical Center Göttingen)

Prof. Dr. Micheal Hörner

(Department of Cellular Neurobiology, Johann-Friedrich-Blumenbach-Institute for Zoology and Anthropology, University of Göttingen)

Camin Dean, Ph.D.

(Laboratory of Trans-synaptic Signaling, European Neuroscience Institute Göttingen)

Date of oral examination: $12^{\text {th }}$ of May, 2016 


\section{Declaration}

I hereby declare that this thesis entitled "Orientation and organization of the presynaptic active zone protein Bassoon: from the Golgi to the synapse" has been written independently and with no other sources and aids than quoted.

Tina Ghelani,

Göttingen, $8^{\text {th }}$ of August, 2016 


\section{Summary}

Neurotransmitter release sites at the presynaptic terminus membrane, known as the active zones (AZs), are surrounded by synaptic vesicle pools and a dense network of five cytomatrix of the active zone (CAZ) proteins. At CNS synapses, Munc13s, RIMs, CAST/ELKS, Bassoon, and Piccolo predominantly form the CAZ scaffold, and together have been shown to promote short- and long-term plasticity by binding to $\mathrm{Ca}^{2+}$ channels and enabling priming and docking of synaptic vesicles to the presynaptic membrane. Even though the components of the CAZ are known, how they are exactly assembled opposite postsynaptic specializations is not yet understood.

It has been shown that AZ proteins (AZPs) are transported on 80nm densecore vesicles called Piccolo/Bassoon transport vesicles (PTVs), to synapses in aggregates together with synaptic vesicles. In addition, Golgi-derived AZ precursor vesicles that transport these proteins have been reported to take different paths out of the Golgi and carry only a small subset of AZPs, although how all AZPs reach and generate a complete and functional AZ at the presynaptic terminus is still under investigation. These observations suggest traffic of a range of different transport vesicles, carrying subsets of AZPs to synaptic sites, and indicates that the mechanisms influencing the final assembly of AZPs at the presynaptic terminus may be predetermined as early as their sorting and loading onto transport vesicles at the Golgi.

To address this hypothesis, this study, examines the localization of endogenous AZPs, using super-resolution microscopy, for the first time at Golgi substructures, the soma, and in the developing axons of hippocampal neurons. AZPs are specifically localized at and around their respective Golgi lamella, in a range of signal sizes that correspond to different loaded transport-carrier types, and present low co-localizations, with one another, in developing axons. This distribution signifies the importance of early sorting and loading of preassembled AZP subsets in the soma. In order to understand the underlying mechanisms that dictate the specific localization of CAZ proteins, a detailed study of the nanostructural orientation and organization of AZPs, at different cellular locations, is required, but hampered by the limitations imposed by the use of primary and secondary antibodies. To overcome this technical limitation, I introduce, characterize, and use new fulllength second-generation Bassoon constructs that are optimized, with respect to their targeting behavior in neuronal cells, and are endowed with tags that can be detected with very small camelid antibodies, so called nanobodies, for super-resolution imaging.

Bassoon is one of the largest CAZ proteins and among the first AZPs to be incorporated at young synaptic sites. It is known to bind to other $A Z$ proteins 
in the CAZ scaffold, and provides structural stability to the CAZ scaffold by downregulating local ubiquitination. This suggests a central role of Bassoon in CAZ scaffold generation. In addition, Bassoon is also the mammalian AZP with the largest cohort of mutant and full-length constructs available, making it an ideal candidate for this study.

STED and FLIM imaging show that full-length Bassoon molecules possess an open and extended conformation at the TGN and are organized 6-20nm from the TGN with neighboring $\mathrm{N}$-termini of molecules in close proximity to each other. Further, these studies show that the first 94 amino acids of Bassoon's N-terminus, but not its myristoylation motif, determines its correct subcellular localization to the TGN, while Bassoon's CC2 domain is sufficient for recruiting the protein to the Golgi. A novel conformation change is observed as the Bassoon molecule travels from the Golgi to synaptic sites, where the molecule appears to lose its extended conformation during trafficking on ChromograninA-positive PTVs, and returns to its extended orientation at synaptic sites. Within these sites, in CAZ scaffold, Bassoon molecules have been previously shown to be oriented with their $\mathrm{N}$-termini extending $80 \mathrm{~nm}$ into the presynaptic bouton and their C-termini positioned around $50 \mathrm{~nm}$ from the presynaptic plasma membrane. In this study I show that the $\mathrm{N}$-termini of neighboring Bassoon molecules are organized in close proximities of $\geq 5 \mathrm{~nm}$ from each other. This result suggests that the organization of Bassoon molecules within the CAZ scaffold closely resembles triangular dense projections regularly observed in EM images of CNS presynaptic sites. Therefore the orientation and the organization of Bassoon molecules promotes structural stability by inhibiting localized ubiquitination and forms the backbone that other AZPs bind to within the CAZ scaffold.

In conclusion, the data reported here suggest that the orientation and organization of Bassoon molecules plays an important role in promoting local subcellular mechanisms from influencing its localization and sorting at the TGN to providing structural stability to the CAZ scaffold, while its change in conformation on its journey, to the CAZ, highlights the first step in understanding the sequence of mechanisms involved in mammalian $A Z$ assembly and synapse maturation.

Keywords: Bassoon; orientation; super-resolution imaging; AZ assembly; cytomatrix of the active zone; STED; FLIM 


\section{Table of contents}

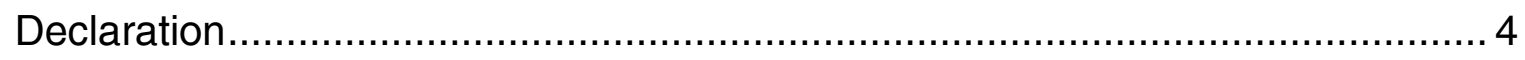

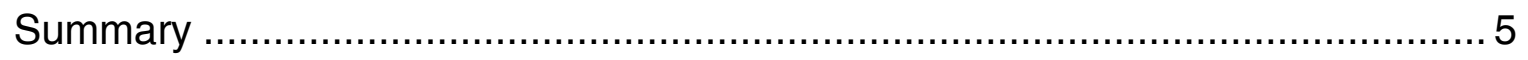

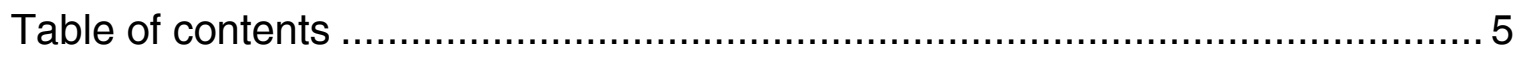

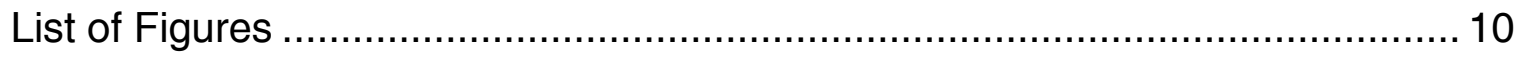

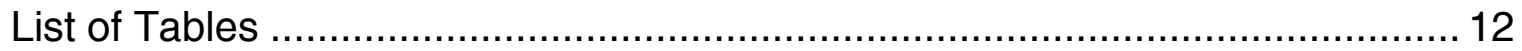

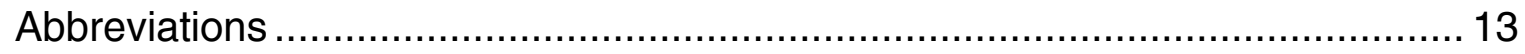

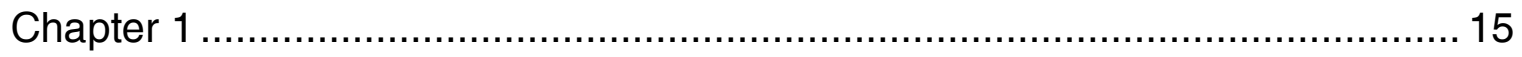

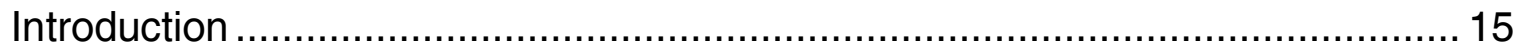

1.1. The active zone (AZ) and its CAZ scaffold...................................... 17

1.1.1. Role of CAZ scaffold protein Bassoon ............................................ 19

1.2. Members of the CAZ scaffold in central synapses ............................. 22

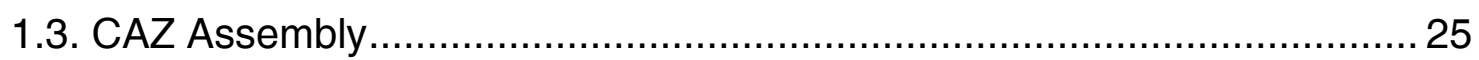

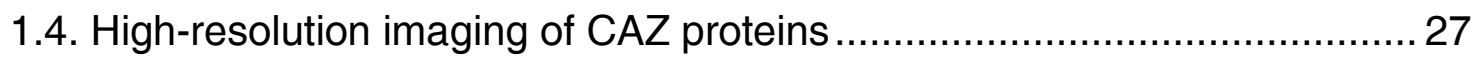

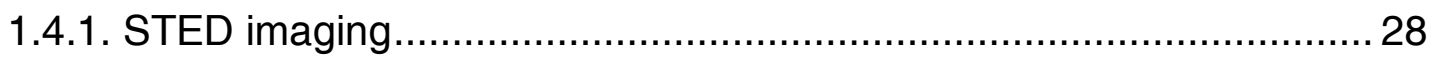

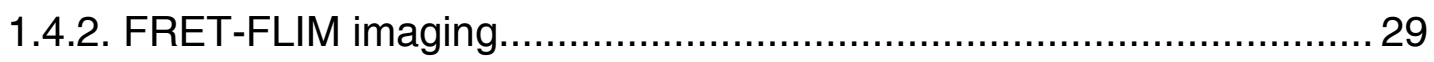

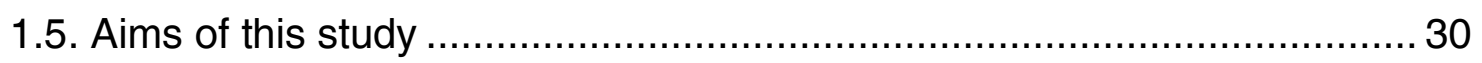

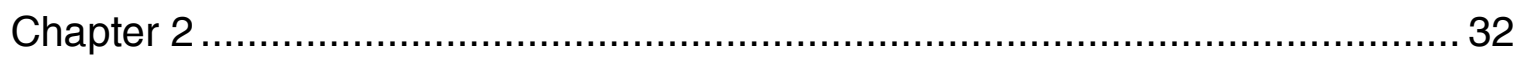

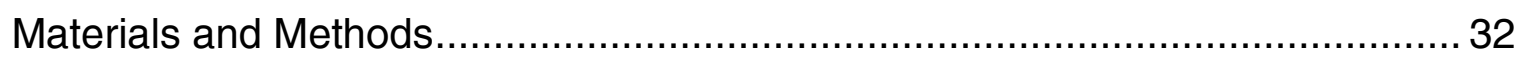

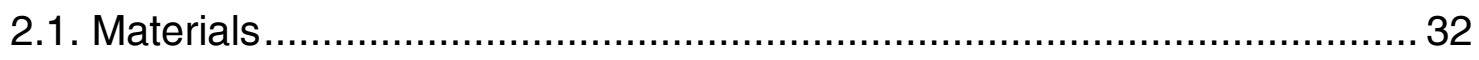

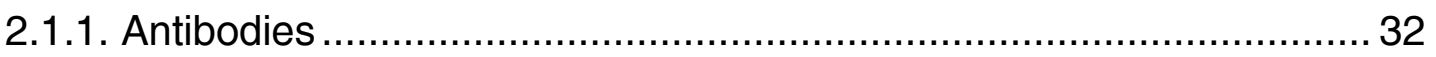

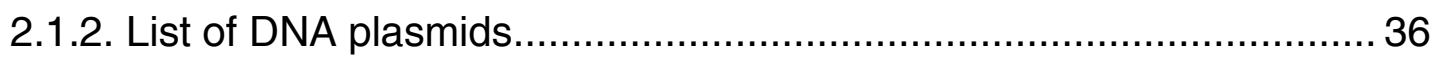

2.1.3. Bsn-/- knockout mouse ………....................................................... 37

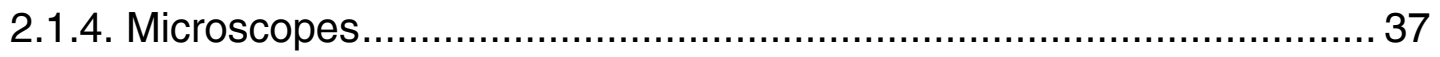

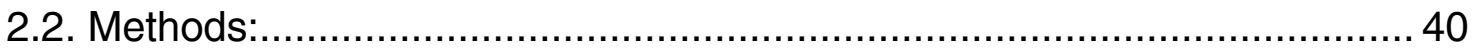

2.2.1. Primary culture of rat and mouse hippocampi.................................. 40 
2.2.3. Transfection methods ................................................................ 43

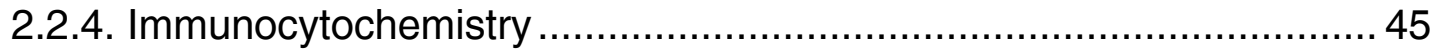

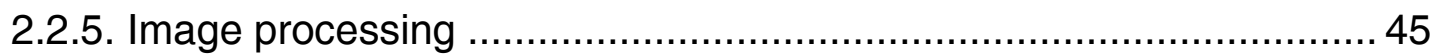

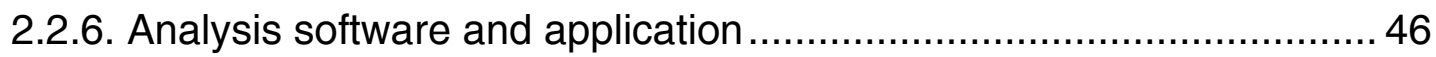

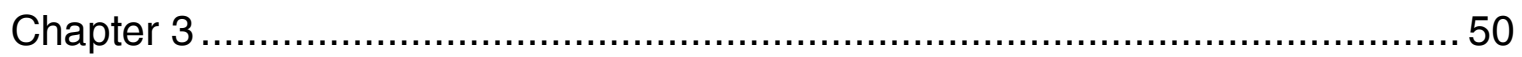

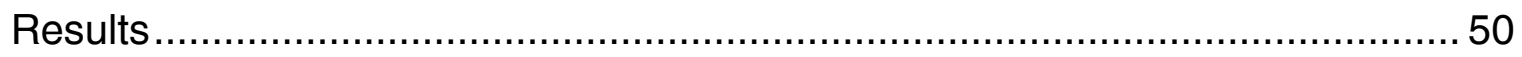

3.1.1. Localization of AZPs to Golgi sub-compartments with and without a $19^{\circ} \mathrm{C}$ block (confocal) 51

3.2. Transport and localization of endogenous and recombinant Bassoon

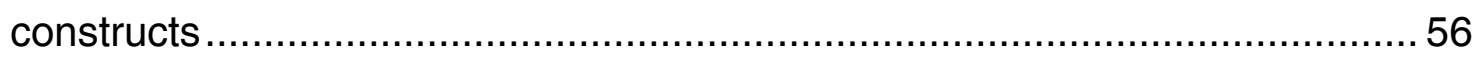

3.2.1. Transport entities generated at the soma …….............................. 57

3.2.2. Distribution of endogenous Bassoon to other AZPs in axons of

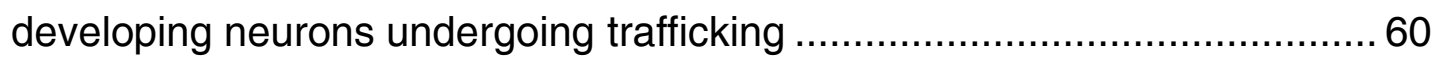

3.3. Characterization of new full-length Bassoon constructs..................... 64

3.3.1. Characterization of full-length Bassoon constructs at the Golgi ......... 66

3.3.2. Characterization of full-length Bassoon constructs at synaptic sites.. 68

3.3.3. Characterization of full-length Bassoon myristoyl mutant at the Golgi

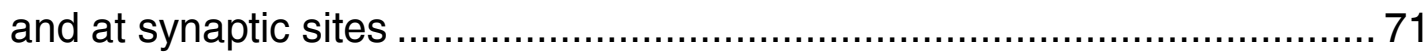

3.4. Super resolution localization of AZPs with nanobodies ....................... 73

3.4.1. Nanobody approach of the localization of tagged proteins (STED) ...73

3.4.2. Localization of tagged AZPs at their respective sub-compartments (STED) 75

3.4.3. Localization of full-length Bassoon construct at neighboring Golgi

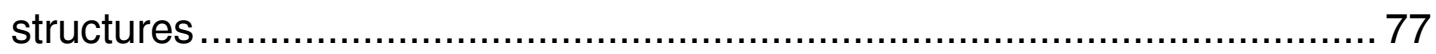

Orientation and organization of the Bassoon molecules ………................... 79

3.5. Localization of full-length Bassoon constructs to the trans-Golgi network 80 3.5.1. Orientation of full-length double-tagged construct at TGN38 ............ 80 3.5.2. Orientation of full-length single-tagged constructs at TGN38............ 83 
3.5.3. Orientation of full-length single and double-tagged constructs at Syn6 84

3.5.4. Detailed orientation of Bassoon molecule at the trans-Golgi network 87

3.5.5. Localization of Bassoon mutants at the trans-Golgi network .............91

3.6. Orientation of Bassoon during PTV transport ........................................ 98

3.6.1. Orientation of the $\mathrm{N}$ - and $\mathrm{C}$ - termini of Bassoon around chromogranin A positive transport packets 99

3.7. Organization of Bassoon molecules at the Golgi and synaptic

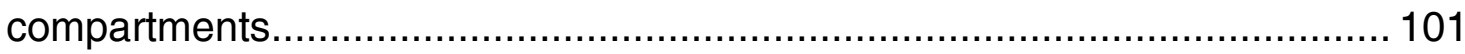

3.7.1. Organization of Bassoon molecules in the soma ........................... 102

3.7.2. Organization of Bassoon molecules at the synapse ....................... 104

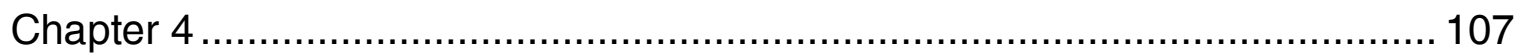

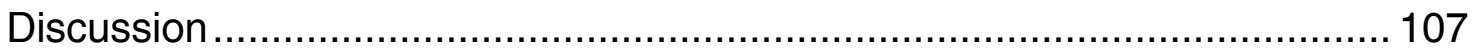

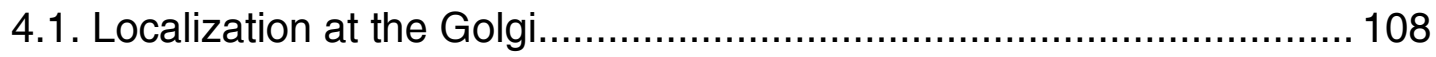

4.2. Recruitment and sorting of AZPs at the TGN.................................. 111

4.3. Transport of AZPs from the soma to nascent presynaptic sites .......... 114

4.4. Orientation of the Bassoon molecule ............................................... 116

4.5. Organization of Bassoon molecules at the TGN and presynaptic sites 121

4.6. Perspectives and open questions ................................................. 124

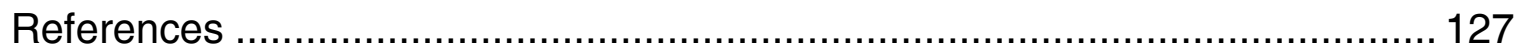

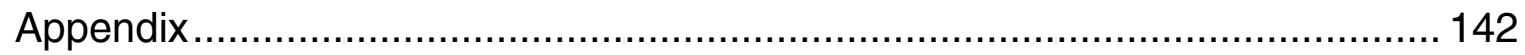

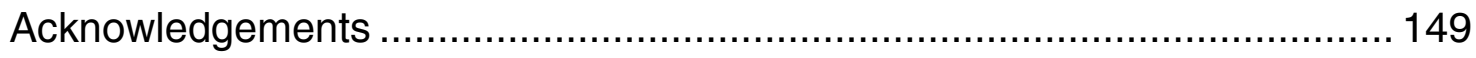

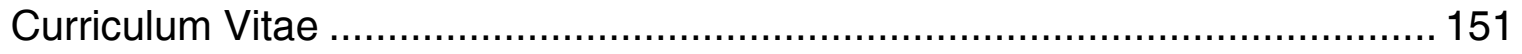

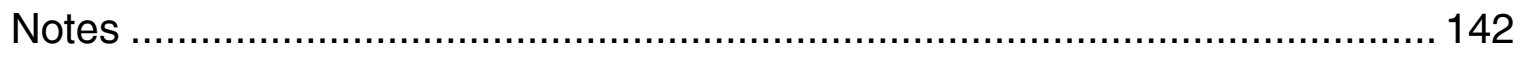




\section{List of Figures}

Figure 1: Active zone ultrastructures of vertebrate and invertebrate neurons..... 18

Figure 2: Ultrastructural and schematic organization of AZPs and SV pools of central synapses.................................................................... 25

Figure 3: Bassoon, Piccolo and Munc13-1 associate with specific Golgi subcompartments.

Figure 4: Distribution and localization of AZPs to their respective Golgi subcompartments with and without a $19^{\circ} \mathrm{C}$ block using STED microscopy. ....54

Figure 5: Size populations of endogenous Bassoon, Piccolo, and Munc13-1 signals at their Golgi sub-compartments.

Figure 6: Early localization of recombinant Bassoon and Munc13-1 to dense-core vesicle marker: Synaptotagmin4 (Syt4).

Figure 7: Distribution of Bassoon, Piccolo and Munc13-1 in developing neurons with epifluorescence microscopy.

Figure 8: Distribution of Bassoon, Piccolo and Munc13-1 during high neuronal trafficking using STED microscopy

Figure 9. A schematic of the second-generation Bassoon constructs used in this study. 65

Figure 10: Recombinant Bassoon localizes closely with the TGN38 in young neurons.

Figure 11: Double-tagged recombinant Bassoon colocalizes with synaptic markers in adult neurons.

Figure 12: Single-tagged recombinant Bassoon also colocalizes with synaptic markers in adult neurons.. 70

Figure 13: Mutation of the myristoyl group of Bassoon does not impede the normal Golgi and synaptic localization of the protein in young and adult neurons.

Figure 14: Using GFP and RFP specific nanobodies to visualize of single molecules of tagged Bassoon protein under $30 \mathrm{~nm}$ resolution. 74

Figure 15: Super resolution localization of GFP tagged Bassoon (95-3938) and full-length Munc13-1 at their respective Golgi sub-compartments. 76 
Figure 16: Full-length Bassoon localizes specifically to the trans-Golgi network compartment instead of the trans-Golgi sub-compartment. .78

Figure 17: Orientation of mRFP-Bsn-mEGFP at the trans-Golgi network. 82

Figure 18: Orientation of mRFP tagged full-length Bassoon constructs at the trans-Golgi network (TGN). 84

Figure 19: Orientation of single and double-tagged full-length Bassoon constructs at the trans-Golgi network marker: Syntaxin 6 (Syn6). 86

Figure 20: FLIM imaging of $\mathrm{N}$-terminus of Bassoon to TGN38 to visualize interaction within $5 \mathrm{~nm}$. 88

Figure 21: A summary of the orientation of full-length Bassoon molecules at the trans-Golgi network 90

Figure 22: Orientation of first-generation mutant Bassoon constructs at the transGolgi network. 92

Figure 23: Comparing the orientation of the double-tagged myristoyl mutant Bassoon construct to the double-tagged full-length the trans-Golgi network. 95

Figure 24: Orientation of G2A-mRFP-Bsn-mEGFP myristoyl mutant construct in endogenous Bassoon-free $\mathrm{Bsn}^{-/}$knockout mice and their $\mathrm{Bsn}^{+/+}$wildtype littermates.

Figure 25: Orientation of full-length single-tagged Bassoon constructs to chromogranin A (CGA) positive PTVs in the soma and down the axon....... 99

Figure 26: Organization of neighboring Bassoon molecules at the soma......... 103

Figure 27: Organization of Bassoon molecules at synaptic sites. 105

Figure 28: A diagrammatic representation of AZPs localizations to different Golgi subcompartments. 110

Figure 29: Structure and orientation of Bassoon and Piccolo proteins at the presynaptic terminus.

Figure 30: A diagrammatic representation of the orientation and organization of tagged Bassoon molecules. 


\section{List of Tables}

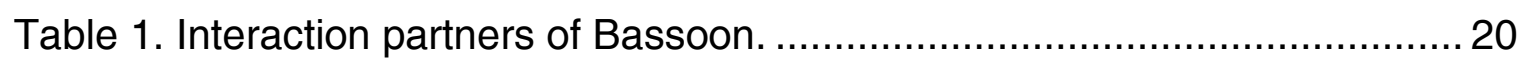

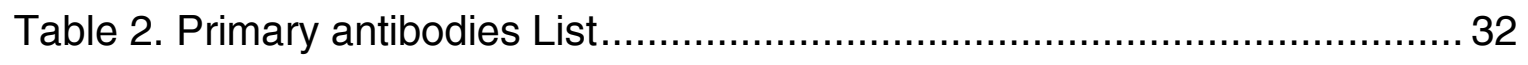

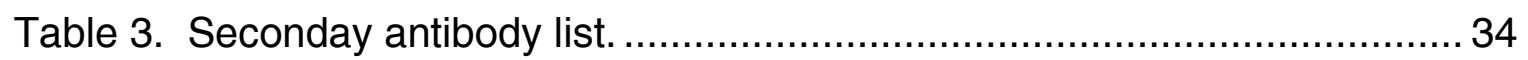

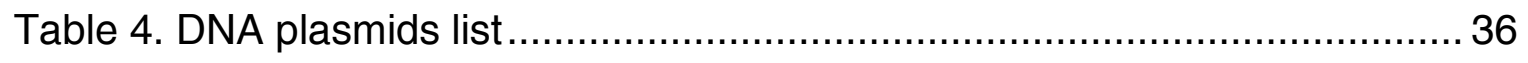

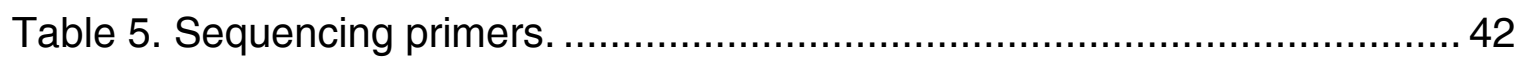




\section{Abbreviations}

AZ: Active Zone

AZP: Active Zone Proteins

CAZ: Cytomatirx of the Active Zone

PSF : Point Spread Function

FWHM : Full Width at Half Maximum

STED : Stimulated Emisson Detection Microscopy

STORM: Stochastic Optical Reconstruction Microscopy

FLIM : Fluorescence-lifetime imaging microscopy

FRET : Förster resonance energy transfer

CGA: ChromograninA

Syt4: Synaptotamin4

a.a. : Amino acid

CC2: coiled-coil 2

BsnGBR : Bassoon Golgi Binding Region

$\mathrm{KO}$ : Knock-out

WT Wildtype

LUT: Look up table

GFP : Green fluorescence proteins

RFP : Red fluorescence proteins

SV: Synaptic vesicle

DCV: dense-core vesicles

PM: Plasma membrane

DP: Dense projection

EM : electron microscope

SVs : synaptic vesicles

DCVs: dense-core vesicles

PTVs: Piccolo-Bassoon transport vesicles

neuromuscular junctions (NMJ)

RIM: Rab-interacting molecules

VGCCs: voltage gated calcium channel 



\section{Chapter 1}

\section{Introduction}

Synapses are specialized sites of contact between two neurons, designed for rapid communication via the chemical release of neurotransmitters from the presynaptic neuronal partner to the postsynaptic neuron. The term synapse was first coined in 1897 by Sir Michael Foster and Sir Charles Scott Sherrington, and based in part on both popular and contradictory theories of nervous system organization ${ }^{1}$, of the time, i.e. the Reticular model (supported by Camillo Golgi's work) and the Neuron Doctrine (based on Santiago Ramón y Cajal's findings). Both forefathers of modern neuroscience performed histological studies of subsets of neurons and their observations were formulated into the two models ${ }^{2}$.

The reticular model postulated by Joseph von Gerlach in 1872, describes the nervous system as a continuous syncytial reticulum consisting of nerve fibers, dendrites, and neurons, nourished through their cell somas, and directly connected to each other over cytoplasmic bridges ${ }^{3}$. While the Neuron Doctrine, formulated by Waldeyer-Hartz in 1891, states that the nervous system is not a continuous reticulum of tissue, but rather consists of separated discontinuous units or cells that became known as neurons. These cells were described as consisting of three main subcellular areas: the soma, fine tree-like processes known as dendrites, and a single long axon ${ }^{4}$. This model was further added to by the law of dynamic polarization, that states that neuronal signals only travel in one direction in neurons i.e., from dendrites, through cell bodies, down axons to the synapse ${ }^{5}$. 
This was an exciting time in neuroscience history where based on purely structural histological data, the basic understanding of the nervous system was deciphered. The next decade saw a range of physiological and biochemical experiments that described the chemical nature of synaptic signal transmission, but it was not until after the development of the electron microscope (EM) in 1933, that the fine structure of synaptic organization was revealed. The first EM images of the synapses were taken in $1953^{6}$, followed very shortly thereafter by a range of EM studies showing electron-dense regions at the pre- and postsynapse, and a synaptic cleft separating these structures. This confirmed the Neuron Doctrine, and the presence of secretory vesicles, now known as synaptic vesicles (SVs), in the presynaptic terminus (Figures 1D and 2A). These vesicles were postulated to contain neurotransmitters and explained the previously described quantal release of neurotransmitters at the synapse observed by Sir Bernard Katz and colleagues at about the same time in the early $1950 \mathrm{~s}^{7}$.

Although the growing number of EM studies provided a wealth of new information about the ultrastructure of the synapse, these alone were unable to identify the molecular components and proteins that constituted the electron-dense regions on both sides of the synapse.

The last 50 years have seen the application of genetic, biochemical, molecular biological and genomic methods to uncover a large number of proteins, in different model organisms, that constitute the composition of the synaptic apparatus. The synaptic proteins discovered included a range of secretory vesicles transporting synaptic vesicle proteins, peptides involved in vesicle docking and fusion, receptors of neurotransmitters, ion channels, enzymes that regulate processing, and of course the neurotransmitters themselves to the plasma membrane. In addition a range of extracellular matrix proteins, cellular signaling proteins, cell adhesion molecules that promote neuronal contacts, cytoskeletal proteins that form the backbone structure of synaptic apparatus and a number of scaffolding proteins that help mediate the structural organization of the different classes of proteins on both sides of the synapse were identified ${ }^{2}$.

This combination of proteins equips the presynaptic terminal for regulated depolarization and calcium-dependent exocytosis of neurotransmitter from synaptic vesicles, and the postsynaptic terminus for neurotransmitter detection by clustering of neurotransmitter receptors. Synapses can be found along the axon of a neuron at presynaptic sites known as en passant boutons or at the axonal distal end known as boutons terminaux ${ }^{8}$. 
These boutons contain resting and recycling pools of synaptic vesicles, as well as a network of cytoplasmic scaffolding proteins known as cytomatrix of the active zone (CAZ) that tether and organize proteins and vesicles required for neurotransmitter release, around roughly 500nm-long specialized regions on the plasma membrane known as the active zone (AZ). The presynaptic membrane of $A Z s$ possesses synaptic vesicle sorting and fusion machinery proteins, and is the site of neurotransmitter release.

\subsection{The active zone (AZ) and its CAZ scaffold}

The CAZ scaffold of presynaptic proteins is observed as the electron-dense structures in EM images, and this structure is organized exactly opposite the postsynaptic scaffold. The most important function of the CAZ is to restrict neurotransmitter release to $A Z s$ and regulate its properties. The CAZ scaffold organizes and separates neighboring AZs and vesicle docking sites while providing a protein network for proteins lacking transmembrane regions, which cannot integrate into the presynaptic plasma membrane, to localize in close proximity to their binding partners and the plasma membrane ${ }^{9,10}$. The CAZ functions to support the structure and function of the $A Z$ site by modulating synaptic vesicle pools by influencing their recruitment, priming, and docking ${ }^{11}$. In addition, its components have also been shown to regulate the organization of $\mathrm{Ca}^{2+}$-channels in the plasma membrane, which indirectly influences and connects the $\mathrm{Ca}^{2+}$ influx within the terminus and to the balance of exo- and endocytic events of the readily-releasable pool (RRP) of synaptic vesicles ${ }^{12}$.

Different $A Z$ sizes, organizations and CAZ protein compositions exist and have been studied in detail in invertebrate Caenorhabditis elegans (C. elegans), Drosphila melanogaster, and vertebrate mice and rat animal models. CAZ composition is species-specific, although $A Z$ size and the CAZ organization in these $A Z$ is influenced by the synapse size, its morphology and the propensity of its function. For example, neuromuscular junctions (NMJ) are large synapses that possess thousands of SVs at elaborately organized AZs and PSDs and are programmed to ensure precise and reliable signal inputs for muscle contractions to occur with high fidelity. On the other hand, central nervous system synapses, such as glutamatergic hippocampal neurons have smaller AZs and less robust neurotransmitter signaling, which allows these neurons to modulate their functional propensity and allows these neuronal networks to have synaptic plasticity $^{10,13,14}$.

The central synapses have simple AZ structures characterized by electron-dense projections (DPs) that define their CAZs, which connect docked SVs and the readily releasable pool (RRP) of SVs via fine filamentous projections and tether them close to release sites on the plasma membrane. Vertebrate NMJ AZs share are similar to drosophila NMJs and are large structures with a linear organization of SV layers that are orchestrated through different filamentous structures, which 
tether the vesicles to the PM. Drosophila NMJ AZs are also called T-bars, describing the meshwork of filament tethers that stretch out to form a consolidated dense site on the plasma membrane into a horizontal platform that organizes the SV layers. C. elegans NMJ AZs although large in size have an AZ structure similar to classical vertebrate central synapses. The most complex vertebrate $A Z$ structures are seen at photoreceptor cell ribbon synapses. These synapses have an elongated AZ structure at the PM, known as an "archiform density", which is attached to a long horizontal filament known as the synaptic ribbon. The ribbon tethers SVs all around it and brings them in close vicinity to the $A Z$ site $^{13}$ (Figure 1).
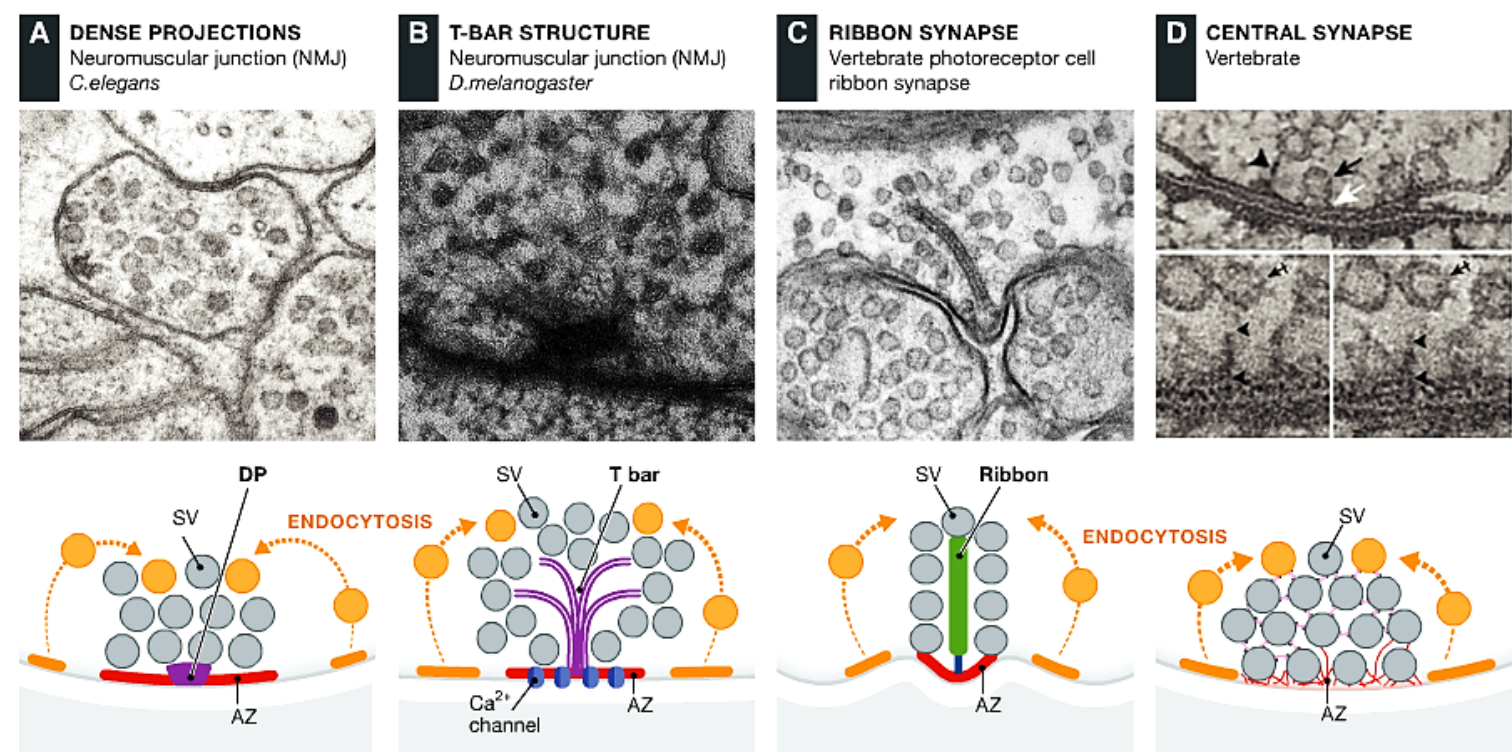

Figure 1: Active zone ultrastructures of vertebrate and invertebrate neurons. Taken from Ackermann et al, 2015 shows the different AZ structures of NMJ, photoreceptor, and central nervous system synapses of vertebrates and invertebrates. The $A Z$ structures show the arrangement of dense-electron projections of the CAZ proteins and the SVs at presynaptic termini of these different synapses.

Vertebrate AZs and invertebrate AZs have many features and variants of core CAZ components in common. Although a greater molecular diversity of vertebrate $C A Z$ proteins, in the form of splice variants and gene duplications, is present to support the functional diversity of different types of AZs and the modulation of synaptic plasticity in a network of central nervous system (CNS) neurons.

Five prominent core CAZ proteins have been identified in the vertebrate synapses namely Bassoon and Piccolo, Rab-interacting molecules (RIMs), Munc$13 \mathrm{~s}$ and $\mathrm{ELKSs} / \mathrm{CAST}^{11}$. The invertebrate Drosophila genome has similar orthologs to vertebrate CAZ proteins. For instance drosophila CAZs have Fife (a Piccolo homolog), Bruchpilot (an ortholog of CAST), DUNC-13 (replaces Munc13), and DRIM (Drosophila RIM) ${ }^{15-18}$. A Bassoon homolog has not yet been identified in Drosophila. Similarly the CAZ composition of C. elegans, comprises of SYD-2, Liprin-a, ELKS-1, UNC-10/RIM, and UNC-13 (Munc-13 variant) ${ }^{19-21}$. 


\subsubsection{Role of CAZ scaffold protein Bassoon}

Bassoon is the second largest CAZ protein, weighs $\sim 420 \mathrm{kDa}$ and forms a, multidomain protein. It was first identified in a rat cDNA screen of synaptic junctions and found to localize in the synaptosomal and synaptic junctional fractions but not in the soluble and myelin-sheath protein fractions. The Bassoon gene is localized on chromosome $9 \mathrm{~F}$, possess a $13 \mathrm{~kb}$ coding sequencing which consists of 10 exons, with more than half of it sequence encoded from exon ${ }^{22}$. Bassoon is one of three vertebrate specific presynaptic proteins, and coincidently shares a large amount of homology, in the form of homology domains, with one of the other two remaining vertebrate specific $A Z$ proteins (AZPs), that is also a key CAZ protein; Piccolo ${ }^{22,23}$. In silico predictions have estimated Bassoon and Piccolo possess an $80 \mathrm{~nm}$ stretched-open, filamentous structure that is rich in prolines and glycines, which promotes their structure, although both proteins have several highly compact regions in their structure ${ }^{24}$. These compact regions form the two N-terminal zinc-finger domains, three coil-coil domains that Bassoon and Piccolo share in homology but use to interact with various secretory, transport, and synaptic proteins. In general, the $\mathrm{N}$-terminus of Bassoon contains the zinc fingers that inhibit the local ubiquitination activity of seven in absentia homolog 1 (Siah1) and promotes synaptic stability ${ }^{25}$. The central CC2 domain region promotes sorting and transport regulatory mechanism as it is flanked by a CTBP binding site (may be involved in Bassoon sorting at the TGN and balancing its expression) ${ }^{26-28}$, and a dyein-light chain binding site (mediates retrograde transport of Bassoon and Piccolo vesicles) ${ }^{29}$, while the CC2 domain itself is the oligomerization site of Bassoon and Piccolo molecules and might promote assembly of AZPs at either the Golgi or at the $A Z^{30-32}$. On the other hand, the Cterminal region of Bassoon possess binding sites for a large range of synaptic AZPs such as CAST ${ }^{26,31,33}$, Munc-13 ${ }^{31}, \mathrm{RIMs}^{34}$, RIM-binding proteins ${ }^{34}$, voltage gated calcium channels (VGCCs) ${ }^{35}$, enzymatic activity regulator D-amino acid oxidase $^{36}$, and SV protein Mover ${ }^{37-39}$. The interaction partners of Bassoon are in more detail described in Table 1.

Through these multi-domain binding sites and its extended structure Bassoon integrates into various mechanisms at play in the presynaptic terminus. These mechanisms include, organization of neurotransmitter release sites by influencing calcium channel localizations, bringing CAZ scaffold proteins and SV pools in close proximity to these sites, maintaining the structural stability, and synaptic integrity, while modulation the local synaptic and synapto-nuclear signaling pathways at play around the $A Z^{40}$.

To understand the exact cellular mechanisms that Bassoon is involved in that regulate presynaptic transmission, a range of studies have been performed using deletion mutants of Bassoon. A partial deletion mutant Bsn ${ }^{\Delta \mathrm{E} 4 / 5}$ generated by deleting most of exons four and five of Bassoon, did not influence synaptic 
transmission of intact cultured hippocampal neurons, although a larger number of silent synapses were generated in $\mathrm{Bsn}^{\Delta \mathrm{Ex} 4 / 5}$ mice $^{22}$. Similar results were observed in another study that performed a shRNA-mediated knockdown of Bassoon in autaptic hippocampal cultures ${ }^{23,41}$. Studies of the $\mathrm{Bsn}^{\Delta \mathrm{Ex} 4 / 5}$ mutant in the high throughput synapses of cerebellar mossy fibers showed that Bassoon is involved in synaptic vesicle replenishment. This mutant and a full knockout of Bassoon showed that the loss of Bassoon caused slowed vesicle recycling and produced a stronger depression in during high-frequency signal transmission ${ }^{42}$. In addition, Bassoon deficient photoreceptor and inner hair cells neurons present a dramatic loss of ribbon synapses and their associated proteins, similar to the loss of SV around T-bars of Bruchpilot deficient drosophila $\mathrm{NMJ}^{43,44}$. In inner hair cells, the loss of ribbon synapses, that float into the cytosol, of Bassoon deficient mice severely affect the synchronous compound activity of the auditory nerve; therefore Bassoon is essential for normal hearing in these mice ${ }^{44}$. These results show that Bassoon molecules may behave as tethers for SVs in different AZs and/or that they emphasize the role of vesicle tethers in regulating vesicle replenishment ${ }^{45}$.

Since its discovery in 1998, the extensive number of Bassoon studies show that there is no clear unifying role of Bassoon in vertebrate central, NMJ and sensory ribbon synapses, as Bassoon seems to have different roles in these synapses, although it is clear that irrespective of its exact role in the different $A Z s$ it promotes the presynaptic activity at specialized $A Z$ sites ${ }^{46}$. 
Table 1: Interaction partners of Bassoon. Modified from Gundelfinger, 2016. Abbreviations: Bsn, Bassoon; Pclo, Piccolo; CC1-3, coiled-coil regions; mouse: (ms) Znf: Zinc-finger domain

\begin{tabular}{|c|c|c|c|c|}
\hline $\begin{array}{c}\text { Bassoon } \\
\text { binding site }\end{array}$ & $\begin{array}{c}\text { Interaction } \\
\text { partner }\end{array}$ & $\begin{array}{l}\text { Description/ } \\
\text { Potential function }\end{array}$ & Reference & $\begin{array}{l}\text { Cellular } \\
\text { process }\end{array}$ \\
\hline $\begin{array}{c}\text { Pclo, Znf1 } \\
521-582 \\
\text { Pclo, Znf2 } \\
1010-1071 \\
\text { Bsn, Znf1 } \\
162-225 \\
\text { Bsn, Znf2 } \\
\text { 459-523 (rat) }\end{array}$ & $\begin{array}{l}\text { Siah1, seven in } \\
\text { absentia homolog } 1\end{array}$ & $\begin{array}{l}\text { E3 ubiquitin ligase, } \\
\text { ubiquitinates SV } \\
\text { proteins, component } \\
\text { of the ubiquitin- } \\
\text { proteasome system. }\end{array}$ & $\begin{array}{l}\text { Waites et al. } \\
(2013)\end{array}$ & $\begin{array}{l}\text { Protein } \\
\text { turnover/ } \\
\text { degradation }\end{array}$ \\
\hline $\begin{array}{c}\text { Bsn aa } \\
1360-1692 \\
\text { (rat) } \\
\text {, not present } \\
\text { in Pclo } \\
\end{array}$ & $\begin{array}{c}\text { Dynein light chains } \\
\text { Dlc-1, Dlc-2 }\end{array}$ & $\begin{array}{l}\text { Link to dynein } \\
\text { motors, (retrograde) } \\
\text { transport of Piccolo- } \\
\text { Bassoon transport } \\
\text { vesicles }\end{array}$ & $\begin{array}{l}\text { Fejtova et } \\
\text { al. (2009) } \\
29\end{array}$ & $\begin{array}{l}\text { Membrane } \\
\text { trafficking }\end{array}$ \\
\hline $\begin{array}{c}\text { Bsn } \\
\text { CC2 domain } \\
\text { 2088-2563 } \\
\text { (rat) }\end{array}$ & $\begin{array}{c}\text { Bsn CC2 } \\
\text { Pclo CC2 } \\
\text { (aa } 3094-3218 \\
\text { mouse) }\end{array}$ & $\begin{array}{l}\text { Homo-/hetero- } \\
\text { dimerization region, } \\
\text { presumably scaffold } \\
\text { formation, } \\
\text { Golgi-binding domain } \\
\text { of Bassoon }\end{array}$ & $\begin{array}{l}\text { Dresbach et } \\
\text { al. (2006) } \\
\text { Wang et al. } \\
\text { (2009) } \\
\text { Maas et al. } \\
(\underline{2012})^{30-32}\end{array}$ & $\begin{array}{l}\text { Scaffolding and } \\
\text { Assembly of } \\
\text { CAZ core } \\
\text { complex }\end{array}$ \\
\hline $\begin{array}{l}\text { Bsn, CC3(rat) } \\
2933-2995 \\
\text { Bsn, CC3(ms) } \\
2873-3077 \\
\text { Pclo, CC3 (rat) } \\
3601-3960 \\
\text { Pclo, CC3(ms) } \\
3657-3715\end{array}$ & $\begin{array}{l}\text { ERC2/ } \\
\text { ELKS2/ } \\
\text { CAST }\end{array}$ & $\begin{array}{l}\text { Interaction with CAZ } \\
\text { scaffolding proteins. } \\
\text { Potentially involved } \\
\text { in anchoring synaptic } \\
\text { ribbons to the active } \\
\text { zone }\end{array}$ & $\begin{array}{l}\text { Takao-Rikit- } \\
\text { su et al. } \\
\text { (2004), tom } \\
\text { Dieck et al. } \\
(\underline{2005}) \text {, } \\
\text { Wang et al. } \\
(\underline{2009})^{26,31,33}\end{array}$ & $\begin{array}{l}\text { Scaffolding and } \\
\text { Assembly of } \\
\text { CAZ core } \\
\text { complex }\end{array}$ \\
\hline $\begin{array}{l}\text { Bsn, } \\
\text { Ser2845 } \\
\text { (rat) }\end{array}$ & $\begin{array}{l}14-3-3 \eta \\
\text { (and other } \\
\text { isoforms) }\end{array}$ & $\begin{array}{l}\text { Phospho-dependent } \\
\text { regulation of anchor- } \\
\text { ing of Bassoon to } \\
\text { CAZ,Phosphorylation } \\
\text { depends on RSK } \\
\text { family }\end{array}$ & $\begin{array}{l}\text { Schröder et } \\
\text { al. (2013) } \\
47\end{array}$ & $\begin{array}{l}\text { Cellular signal- } \\
\text { ing, scaffolding } \\
\text { and potentially } \\
\text { assembly of } \\
\text { CAZ core } \\
\text { complex }\end{array}$ \\
\hline $\begin{array}{c}\text { Bsn, aa } \\
2715-3263 \\
\text { (rat) } \\
\text { Not tested } \\
\text { for direct } \\
\text { interaction } \\
\text { with Pclo }\end{array}$ & $\begin{array}{l}\text { D-Amino Acid } \\
\text { Oxidase, }\end{array}$ & $\begin{array}{l}\text { Enzyme metabolizes } \\
\text { the NMDA receptor } \\
\text { co-agonist D-serine. } \\
\text { DAO activity } \\
\text { significantly inhibited } \\
\text { by interaction with } \\
\text { Bsn }\end{array}$ & $\begin{array}{l}\text { Popiolek et } \\
\text { al. (2011) } \\
{ }_{36}\end{array}$ & $\begin{array}{l}\text { Cellular } \\
\text { signaling, } \\
\text { Regulation of } \\
\text { enzymatic } \\
\text { activity }\end{array}$ \\
\hline $\begin{array}{l}\text { Bsn, aa 3601- } \\
3820 \text { (C-term) } \\
\text { (mouse) }\end{array}$ & $\begin{array}{c}\text { Munc13 (N-term) } \\
\text { and RIM }\end{array}$ & $\begin{array}{l}\text { Interaction with } \\
\text { presynaptic } \\
\text { scaffolding }\end{array}$ & $\begin{array}{l}\text { Wang et al. } \\
(2009)\end{array}$ & $\begin{array}{l}\text { Scaffolding and } \\
\text { Assembly of } \\
\text { CAZ core } \\
\text { complex, SV } \\
\text { priming }\end{array}$ \\
\hline $\begin{array}{l}\text { Bsn aa 3263- } \\
3938 \text { (rat) } \\
\text { Absent in Pclo }\end{array}$ & Mover/TPRGL & $\begin{array}{l}\text { SV protein, negative } \\
\text { regulator of synaptic } \\
\text { release probability }\end{array}$ & $\begin{array}{l}\text { Kremer et } \\
\text { al.(2007), } \\
\text { Ahmed et } \\
\text { al.(2013), } \\
\text { Korber et al. } \\
(\underline{2015})^{37-39}\end{array}$ & $\begin{array}{l}\text { Potential role in } \\
\text { membrane } \\
\text { trafficking } \\
\text { Regulation of } \\
\text { exocytosis }\end{array}$ \\
\hline
\end{tabular}




\subsection{Members of the CAZ scaffold in central synapses}

\subsubsection{CAZ scaffold protein Piccolo}

Piccolo (also known as Aczonin) is the largest CAZ protein in the scaffold, with a molecular weight of $\sim 550 \mathrm{kDa}$, and it shares with Bassoon a high degree of sequence homology (50 - $80 \%$ common sequence identity), at two N-terminal zinc-fingers and three coil-coil domains together known as the Piccolo-Bassoon homology domains. These domains enable Bassoon-Piccolo interactions at the central CC2 and allow Bassoon and Piccolo complexes to compete for binding with other synaptic and scaffold proteins ${ }^{26}$. For example the $\mathrm{N}$-terminal zinc finger domains of Piccolo and Bassoon bind to Siah1 ${ }^{25}$, though it is not yet understood whether the proteins collaborate or compete to inhibit Siah1 activity, while the C-terminal CC3 domain of Bassoon and Piccolo have been shown to competitively interact with the CC2 domain of $\mathrm{CAST}^{48}$.

Piccolo in addition has some unique interaction domains that separates its function from Bassoon's function and contributes to roles that CAZ scaffold provide towards enabling presynaptic transmission.

The N-terminal zinc-finger domains of Piccolo additionally have $40 \%$ and $39 \%$ homology to the zinc- finger domains of rabphilin-3A and RIM respectively, and contain a PRA1 binding site that has been shown to mediate Piccolo interactions with rab3A and VAMP2 receptors, thus linking Piccolo and synaptic vesicles pools at the $A Z^{49}$. Piccolo possesses a few proline-rich sequences over its structure. A proline patch in the $\mathrm{N}$-terminus of piccolo interacts with actin binding protein 1(Abp1), which links Piccolo by interacting with actin, dynamin ${ }^{50}$, and a GTPase that mediates the fission of SV vesicles ${ }^{51}$.

The central region of Piccolo also possess another proline patch that interacts with profilin, an actin-binding protein, that influences the actin-dynamics within presynaptic terminal ${ }^{52}$. This enables Piccolo to link the CAZ scaffold of proteins to cytoskeleton of the presynaptic terminus as well as incorporate itself into the process of endocytic fission at the AZ site.

Piccolo possesses two C2 domains, namely C2A and C2B domains, within its Cterminus that are not present in Bassoon. The $\mathrm{C} 2 \mathrm{~A}$ domain has been shown to undergo a conformation switch, upon binding $\mathrm{Ca}^{2+}$, that promotes dimerization and $\mathrm{Ca}^{2+}$-dependent phospholipid binding implicating a role for Piccolo in shortterm plasticity ${ }^{53}$. Overall, by virtue of its large size and its CAZ interaction partners, Piccolo incorporates itself into the CAZ scaffold and links the CAZ scaffold the action cytoskeleton and SV recycling events at the presynaptic terminus. 


\subsubsection{Munc-13s, RIMs and CAST/ELKs}

Munc13, the vertebrate specific isoform of unc-13 found in C. elegans, is a small yet integral CAZ protein that interacts and neuronal Rab3 isoforms (RIMs) to regulate presynaptic neurotransmitter transmission ${ }^{54,55,56}$. CAST/ELKs are quintessential CAZ as a scaffold proteins that despite having different roles in different synapses are always involved in organizing the CAZ structure ${ }^{18}$. All three proteins bind to specific C-terminal subdomains of Bassoon and Piccolo and are therefore integral members of the CAZ scaffold ${ }^{31,34,26,31,48,57}$. A schematic depiction of these interactions can be referred to in Figure 2.

Three Munc13 genes exist in the brain Munc13-1, Munc13-2, and Munc13-3, are roughly $\sim 222 \mathrm{kDa}$ in weight and all three isoforms share three evolutionary conserved $\mathrm{C} 2$ domains present in the $\mathrm{N}$ - terminal, central, and $\mathrm{C}$-terminal regions of the protein ${ }^{56,58}$.

The C2 domains of Munc13-1, i.e. C2B and C2C, known as the MUN domain (spans from aa 859-1531), binds to the N-terminus of syntaxin and thereby integrates itself, while linking the CAZ scaffold, to the core of SNARE complex that forms presynaptic membrane machinery at $A Z$ sites ${ }^{55}$. This interaction suggests that Munc13-1 may mediate synaptic vesicles docking at AZs. Additionally, the MUN domain, when expressed in hippocampal neurons lacking Munc13s, rescues the SV priming deficits ${ }^{59}$. SV priming is a maturation step that occurs between the vesicle docking and SNARE-mediated SV fusion steps.

The presence of SV priming is attributed to the combination of opposing electrophysiological and ultrastructural observations noted in Munc13-1 knockout and Munc13-1 and Munc13-2 double knockout mice cultures. Glutamatergic hippocampal neurons of Munc13s, upon inspection for ultrastructural deficits, showed that docked SV vesicles numbers remained unchanged, despite the complete loss of evoked EPSCs in synapses deficient for Munc13s ${ }^{60,61}$. These results suggest Munc13s use their conserved MUN domain to play a crucial role for synaptic vesicle maturation.

The role of Munc13s in SV priming is compounded by the interaction of Munc131's C2A domain with N-terminal zinc-finger domain of RIM. Disruption of this interaction in RIM deficient synapses prevents synaptic vesicles from reaching and fusing with the presynaptic membrane, as the lack of RIM forces Munc13-1 C2A domains to homo-dimerize thereby inhibiting Munc13's SV priming and fusion competence ${ }^{55,62}$. This observation suggests that the $\mathrm{N}$-terminus of RIM is essential for activating Munc13-1 molecules to mediate SV priming.

The RIM protein family consists of neuronal Rab3 isoforms that regulate neurotransmitter release at the presynaptic terminus. RIMs were first isolated and identified in a yeast two-hybrid screen of Rab3C against a rat brain cDNA library, 
and found isolated on the synaptic vesicle fraction. The protein is 1554 amino acids long and consists of an $\mathrm{N}$-terminal zinc finger domain comprised of alpha helices, alanine and proline-rich patches, a postsynaptic density domain, and Cterminal C2A and C2B domains. The RIM protein sequence is well conserved in invertebrates, but in vertebrates at least four RIM genes exist, RIM1-RIM4, with two isoforms each for RIM1 and RIM2 ${ }^{54}$.

The $\mathrm{N}$ terminal alpha helical domain of RIM binds to synaptic vesicle component rab3, forming a tight link between RIMs and $\mathrm{SVs}^{55}$. In addition the central PDZ domain of RIM proteins binds to N- and $\mathrm{P}-/ \mathrm{Q}$-type $\mathrm{Ca}^{2+}$ channels. The interaction RIMs and $\mathrm{Ca}^{2+}$ channels is necessary for recruiting and organizing $\mathrm{Ca}^{2+}$ channels at the AZ. Deletion of the PDZ domain of RIMs not only affects the clustering of $\mathrm{Ca}^{2+}$ channel at the presynaptic membrane, but causes a decrease in $\mathrm{Ca}^{2+}$ influx, which in turn reduces the priming of SVs and thereby fails to interact $\mathrm{Ca}^{2+}$ channels to synaptic vesicles ${ }^{63}$.

CAST or CAZ-associated structural protein, and ELKS (named after its highest a.a. content glutamate $(E)$, leucine $(\mathrm{L})$, lysine $(\mathrm{K})$, and serine $(\mathrm{S})$ ) are $\mathrm{CAZ}$ proteins that are 957 a.a. and 948 a.a.-long, respectively, and share a $71 \%$ a.a. identity ${ }^{64}$. Two isoforms for ELKS: ELKS $\varepsilon$ and ELKS $\alpha$ exist. ELKS $\alpha$ is the neuron specific isoform that is involved in CAZ organization, while ELKS $\varepsilon$ are ubiquitously expressed, do not localize to the CAZ, and have been implicated in GTP-dependent Rab6 interaction that mediates its secretory traffic to membranes ${ }^{64,65}$.

CAST and ELKS $\alpha$ colocalizes with Bassoon in neuronal cultures and possess four coil-coil domains and a C-terminal IWA conserved domains ${ }^{33,64}$. The first two coil-coil domains (spanning 680 a.a. of their $\mathrm{N}$-terminus), are essential for CAST and ELKS $\alpha$ targeting to the CAZ, while the CC2 domain of CAST competitively binds to the CC3 domains of CAZ scaffold proteins; Bassoon and Piccolo ${ }^{48}$. Although knocking out CAST does not yield an effect on synaptic transmission in their central excitatory synapses, it however crucially impairs retinal ribbon synapse transmission. Knockout of the CAST protein produces smaller AZs and diminished transmission in excitatory neurons ${ }^{66}$, and enlarged resting SV pools in inhibitory neuronal terminals, suggesting that CAST influences SV priming ${ }^{67}$.

Together these five AZPs build a network of proteins localized in close proximity to the $A Z$ site, link the site to SVs and prime them, arrange $\mathrm{Ca}^{2+}$ channel to the AZ site, promote the structural stability of scaffold by inhibiting local degradation mechanisms and link the scaffold to the dynamically changing actin cytoskeleton in the presynaptic terminus (Figure 2). 

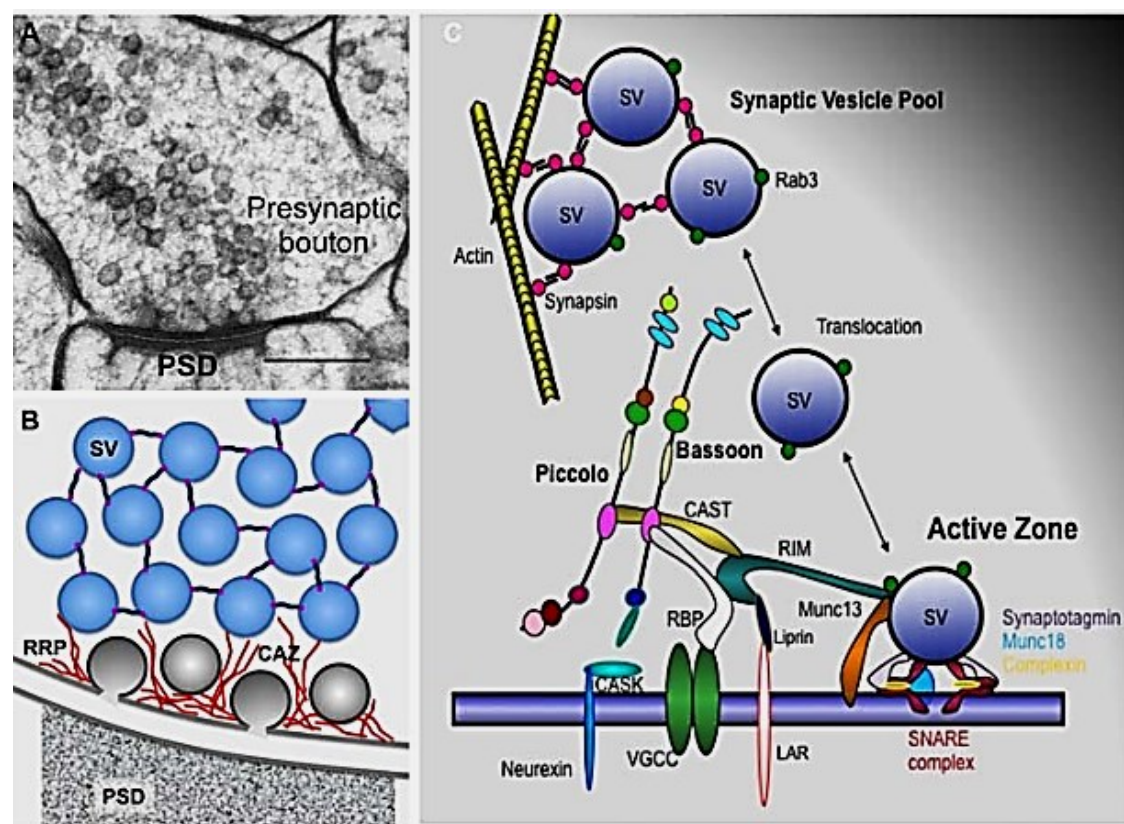

Figure 2: Ultrastructural and schematic organization of AZPs and SV pools of central synapses. Modified from Gundlefinger et al, 2016. (A) Cryo-electron micrograph rat central excitatory synapse (originally published in Rostaing et al., 2006). (B) Schematic organization of SVs pools within presynaptic boutons, the reserve SV pool tethered via synapsin and the docked SV pool are implanted in the CAZ (red tethers). (C) Schematic of CAZ proteins, interaction partners guiding the SV clustering, translocation, docking, priming and fusion at the presynaptic terminus.

Scale bar A, 200nm.

\subsection{CAZ Assembly}

Studies following the discovery of AZPs of CNS synapses initially proposed that a complete complement of AZPs present on a single, $80 \mathrm{~nm}$ in diameter, densecore vesicle (DCV), defined at the Piccolo-Bassoon transport vesicle (PTV), delivers the entire CAZ scaffold to the presynaptic terminus possibly by fusing with the plasma membrane. And evidently so, as an exhaustive set of synaptic proteins (such as VGCCs, SNAREs: syntaxin-1 and SNAP-25, and N-cadherin) and active zone core components Bassoon, Piccolo, RIM1, Munc13, CAST1, and CAZ-associated protein Munc18-1 were isolated in the same insoluble synaptosomal fraction of rat light-brain fractions ${ }^{68,69}$.

Today this view of the PTV model is considered an oversimplification, as recent studies illustrate that the model is a lot more complicated. The most recent study addressing this topic shows that, in fact, the assembly of AZPs may already begin at the trans-Golgi Network (TGN) sorting compartment of the Golgi. This study also highlighted differential sorting of AZPs, as early as their localization to the Golgi, and shows that a subset of Bassoon, Piccolo, and ELKs leave the Golgi on precursors generated at the TGN, while Munc13 is localized to cis-Golgi, and RIM1 is diffused in the cytosol, only to be recruited later during a post-Golgi step $^{32}$. This suggests that preassembly of AZPs occurs in a multistep process, the order of which is as yet unclear, although it may involve a maturation step in 
which PTVs recruit other AZPs on their way to the AZ. The preassembly of AZP subsets, for example for Piccolo-Bassoon-ELK precursors, have been suggested to first assemble at the TGN, and require not only localization and binding to specific Golgi substructures, but an association to each other, and membrane fission and translocation regulators such as CtBP1/BARS $30,32,69,70$

In addition, ultrastructural studies of young hippocampal axons have revealed Bassoon and Piccolo in transit on $\sim 220 \mathrm{~nm} \times 130 \mathrm{~nm}$ transport aggregates, consisting of 1-2 PTVs and 5-6 SVs that co-traffic PTV proteins and SV proteins (such as VAMP2, synaptotagmin, synapsin-1, and SV2), together as a preassembled complex ${ }^{71}$. This clarifies the presence of synaptic proteins on synaptic vesicles and AZPs like Bassoon and Piccolo on PTVs that get isolated in the same biochemical brain fraction, whilst being loaded on different carriers. It also suggests that a certain degree of preassembly may be promoted during transport on such transport aggregates, and that the delivery of such an aggregate may suffice generation of a functional $A Z$ site.

The transport of AZPs has been shown to require microtubule-based transport involving specific retrograde and anterograde transport motors. So far three AZPtransport complexes have been identified, namely Piccolo-Bassoon-ELKs on TGN precursors, Munc13 on cis-Golgi precursors, and RIM-Neurexin-CASKVGCCs on unclassified precursors, that gets associated in a post-Golgi step ${ }^{3,32,71}$. In combination with their distinct transport subgroups, AZPs may also be differentially trafficked based on the motor proteins and adaptor binding sites they possess. For example, Bassoon has been shown to directly interact with dyneinlight chain (Dlc1/2) that mediates its retrograde transport, while PTVs have also been reported trafficking in an anterograde fashion. Anterograde transport of PTVs is mediated via the direct interaction of the PTVs to syntabulin, a kinesin adaptor protein ${ }^{29,72}$. Munc13 and RIM invertebrate-specific isoforms on the other hand have been implicated in an unidirectional kinesin mediated transport ${ }^{21}$. Such variability in transport could be used by the neuron to implement a temporal and sequential traffic of all AZPs to the AZ. Additionally, the incorporation of two to three PTVs, each carrying a unitary load of AZP, has been shown to suffice the generation of a new $A Z$, which might also promote sequential acquisition of the different AZPs into the CAZ scaffold ${ }^{69}$.

How mammalian AZs are assembled is as yet unclear, although the classical PTV model hypothesizes delivery of a complete CAZ scaffold on top of a PTV that fuses with the plasma membrane, and leaves the CAZ proteins behind, at a defined $A Z$ site, to form a quick $C A Z$ scaffold. 
This notion is supported by the presence of visible SV recycling events at the new $A Z$ sites, within a matter of 30 minutes from $A Z$ formation, suggesting that such a quick assembly of $A Z$ sites is fashioned from the delivery of preassembled AZP sets ${ }^{73}$. In addition, it might be possible that a large and single step delivery of preassembled PTV and SV proteins might initiate the formation of a functional mammalian $A Z$, which is unique to only vertebrate synapses, as it involves the delivery of two of the three vertebrate-specific $A Z$ proteins, Bassoon and Piccolo ${ }^{74}$.

Upon delivery to nascent synapses, transsynaptic adhesion molecules Neurexins and Neuroligins signal the site of $A Z$ on the plasma membrane, according to which the CAZ complex builds up exactly opposite the post synaptic scaffold. The mechanisms orchestrating this exact spatial organization of the CAZ scaffold are also not understood ${ }^{75}$. Once at its correct localization in the presynaptic terminus CAZ scaffold proteins interact with each other and the actin cytosketon of the presynaptic bouton to form a dense, insoluble network, from which, an AZP once incorporated cannot be washed out, even with a Triton extraction (Table $1 \&$ ref $^{70}$ ).

\subsection{High-resolution imaging of $C A Z$ proteins}

\section{Ultrastructural studies of CAZ proteins of central synapses}

Since their discovery roughly two decades ago, CAZ proteins of central nervous system synapses have been extensively investigated for their function. A range of ultrastructural studies of single CAZ protein deficient synapses have reported no change in CAZ scaffold structure of central synapses ${ }^{41,42,60,61,63,66}$. Although reduction in populations of clustered SV pools around the CAZ was observed in double knockouts of Bassoon and Piccolo, RIM, and CAST deficient neurons ${ }^{41,42,63,66}$. This suggests that a single mammalian CAZ protein cannot alone cause a loss or change in CAZ scaffold structure. It is therefore essential to study the ultrastructure of all five AZPs in relation to each other, to begin understanding the mechanisms that influence assembly of the CAZ scaffold. With recent advances in new super resolution techniques, it has become easier to study the precise localization of tagged and endogenous AZPs. Two recent studies, using two different super resolution techniques, STORM and EM, have illustrated the specific localizations of $\mathrm{N}$ - and $\mathrm{C}$-termini of Bassoon and Piccolo molecules to RIM, Munc13, and calcium channel localization in the presynaptic terminus of mature synapses. The $\mathrm{N}$-termini of Piccolo and Bassoon extend roughly $80 \mathrm{~nm}$ into the presynaptic bouton, while their C-termini, the central regions of Munc-13-1 and RIM1a, and the cytoplasmic loop of the P/Q type $\mathrm{Ca}^{2+}$ channels localize $20-30 \mathrm{~nm}$ from the presynaptic plasmamembrane ${ }^{76,77}$. 
With the ultrastructural ground work of matured mammalian CAZ structure available, the next questions to address are: 1) how does CAZ assembly occur, and 2) what sort of maturation events do AZPs undergo to attain their final ultrastructural localization in adult neuron. I will therefore, in this study, use STED microscopy and FLIM, and a range of full-length and mutant Bassoon constructs to understand the detailed localization, orientation, and organization of AZPs at different subcellular locations in developing neurons.

\subsubsection{STED imaging}

Imaging subcellular structures below the diffraction-limit of light has become a widespread technique. Super resolution microscopy using stimulated emission depletion (STED) is one such technique that alters the point spread function of traditional excitation laser beam by overlaying a second, doughnut-shaped, redshifted laser beam (the STED beam), which suppresses fluorescence emission of fluorophores located underneath the donut, leaving only the center of the excitation lazer free. The fluorophore suppression is executed through the fundamental properties of stimulated emission, wherein a fluorophore in its excited-state interacts with a photon that possesses the energy difference between the ground and excited state of the fluorophore, through this interaction the excited fluorophore is forced to return to its ground state, before any spontaneous fluorescence emission can occur. The fluorescence of the molecules that fall in the overlap regions of the STED beam and excitation beam are switched off, leaving roughly a $20-50 \mathrm{~nm}$ in diameter point spread function of the center of the excitation beam free to image fluorescence and provide super resolution ${ }^{78}$.

STED microscopy applies the use of further red-shifted wavelengths, with respect to the absorption spectrum of the fluorophores imaged, during stimulated emission to avoid undesired excitation. Similarly, the two-color STED microscopy setups, used in this study, employ a single $775 \mathrm{~nm}$ STED pulsed laser beam, on spectrally distinct red and far-red dyes that are excited and detected in an interleaved fashion to diminish their spectral crosstalk ${ }^{79}$.

STED microscopes have been used to visualize fluorescence-labeled sub-cellular structures in unprecedented detail while permitting the use of simple sample preparation and labeling techniques that employ fluorescent tags and antibodies to visualize locations of biomolecules. These microscopes can resolve single fluorescent molecules at a $20-50 \mathrm{~nm}$ resolution range compared to the $200 \mathrm{~nm}$ resolution of confocal microscopes, although the use of traditional mono- and polyclonal antibodies for STED imaging have been shown to inhibit the complete labeling of molecular epitopes of a protein of interest and limit the resolution obtained from the STED microscopes, due to the large sizes of primary and secondary antibodies complexes generated ${ }^{80}$. Alternatively the use of small, camelid antibodies, comprising of only one heavy chain, known as nanobodies 
have become popular to overcome the problems encountered with application of antibodies $^{81}$. However the generation of nanobodies specific for a new protein of interest is expensive and time-consuming process. The recent success of RFP and GFP specific nanobodies have reduced costs and increased the possibility of super resolution imaging of a large cohort of fluorescent tagged proteins, traditionally used for biochemical assays and live imaging ${ }^{82-84}$.

GFP and RFP nanobodies are small, high-affinity, antibody-fragments generated from a single amino acid chain, off one of the two heavy chains, of the camelid IgG antibody molecule. These antibody fragments are folded into a $\sim 10-15 \mathrm{kDa}$ epitope-binding hypervariable domain of dimensions: $1.5 \mathrm{~nm}$ in diameter and $2.5 \mathrm{~nm}$ in height ${ }^{81}$ and are generated to identify only one specific, threedimensional epitope on top of a RFP or GFP molecule. These nanobodies can be bought pre-coupled to two molecules of secondary ATTO-TEC dyes each with a size of $2.5 \mathrm{~nm}$ making the entire antibody complex roughly $5 \mathrm{~nm}$ and allowing super resolution imaging, at $20 \mathrm{~nm}$ resolution limit, to be accurate and uninhibited. These nanobodies are routinely used to for tagged proteins pulldown assays ${ }^{84}$ and single-molecule localization microscopy techniques ${ }^{82,83,85}$.

In this study I will use full-length and mutant Bassoon constructs tagged with RFP and/or GFP to understand the detailed localization and orientation of the protein in developing neurons.

\subsubsection{FRET-FLIM imaging}

Förster resonance energy transfer (FRET) is a dipole-dipole interaction between a pair of fluorophores that are closely positioned within $5 \mathrm{~m}$ of each other and possess a large spectral overlap between the emission of a donor fluorophore and the absorbance spectrum of an acceptor fluorophore. FRET between donor and acceptor fluorophores quench the fluorescence of the donor, which is proportional to the efficiency of FRET recorded. FRET imaging has been extensively used to study interactions of couplets of proteins of interest in living cells and generate molecular tools in the form of FRET sensors to record various protein activities ${ }^{86}$.

Fluorescence lifetime imaging (FLIM) is a powerful quantitative FRET approach that measures the changes in fluorescence lifetime of the excited state lifetime of the donor fluorophore in the presence of acceptor. The average lifetime of the donor is reduced when the acceptor is in a close enough proximity that permits quenching of the donor and is therefore a direct indicative of FRET ${ }^{87}$.

This method is built around the intrinsic property of the fluorescence lifetime of fluorophores and is therefore independent of fluorophore concentration effects, microscope optical path, and moderate levels of photobleaching, which makes 
the recorded reduction of the donor lifetime an extremely robust and quantitative estimate of the FRET efficiency ${ }^{87}$.

These advantages also promote the use of tagged proteins, that are not necessarily generated as genetically encoded FRET sensors, and are ideal for the study of the same AZP constructs that are also used for STED microscopy. In addition, recent studies show that TagGFP-TagRFP pairs are superior to classical CFP/YFP FRET indicators, which have a lower spectral overlap and require narrow band-pass filters that cause a dramatic loss of emission. The TagGFP-TagRFP pair also have a 1.5-fold higher spectral overlap, compared to similarly separated band-pass filters pair of TagGFP-mCherry ${ }^{87}$. This makes FLIM imaging of readily available GFP- and RFP- tagged AZPs, an ideal choice to studying the orientation and organization of AZPs.

\subsection{Aims of this study}

Little is known about the mechanisms that regulate CAZ assembly and $A Z$ maturation of mammalian synapses. With new advances in super-resolution microscopy techniques allowing more synaptic proteins to be visualized with greater detail, and compared to classical ultrastructural studies, the information describing the detailed localizations of AZPs is slowly being stitched together. However, a comprehensive overview of how the presynaptic scaffold is initiated, assembled, and matured at $A Z$ sites is yet to be provided. There is recent evidence that suggests the delivery of AZPs, on PTVs to the presynaptic scaffold, is through an unclear multistep process ${ }^{32}$. It is therefore possible that assembly and organization of CAZ components may already be pre-programmed and influenced by cellular process, prior to the formation of a matured $A Z$ site and may shape the final organization of AZPs within the CAZ scaffold. To realize this information, a detailed step-by-step localization of AZPs from their first station in the neuronal soma to nascent synaptic sites is required.

In this study, I aim to dissect the detailed localization of AZPs at different subcellular locations in developing neurons. This line of investigation should help reveal molecular mechanisms that dictate the specific localization of CAZ proteins, and improve our understanding of basic cellular process that regulate sorting, trafficking and delivery of synaptic proteins to AZs. To perform these studies, I aim to characterize new full-length Bassoon constructs, optimize them for visualization by super-resolution microscopy techniques, and evaluate them in context of known ultrastructural studies. The application of these constructs provide a timely opportunity to resolve the orientation and organization of Bassoon molecules, and highlight changes in conformation that might link CAZ structure to the underlying mechanisms of CAZ assembly. 


\section{Chapter 2}

\section{Materials and Methods}

\subsection{Materials}

\subsubsection{Antibodies}

\section{Primary antibodies}

Table 2: A selection of primary antibodies used in this study.

\begin{tabular}{|l|l|l|l|l|}
\hline $\begin{array}{l}\text { Primary } \\
\text { antibody }\end{array}$ & $\begin{array}{l}\text { Species } \\
\text { reactivity }\end{array}$ & $\begin{array}{l}\text { Dilution } \\
\text { factor }\end{array}$ & Company & Catalog number \\
\hline Bassoon & guinea pig & 1 to 300 & $\begin{array}{l}\text { Synaptic } \\
\text { systems }\end{array}$ & 141004 \\
\hline Bassoon & mouse & 1 to 500 & $\begin{array}{l}\text { ENZO } \\
\text { lifesystems }\end{array}$ & 04011120 \\
\hline Bassoon & rabbit & 1 to 500 & $\begin{array}{l}\text { Synaptic } \\
\text { systems }\end{array}$ & 141003 \\
\hline Munc13 & rabbit & 1 to 300 & $\begin{array}{l}\text { Synaptic } \\
\text { systems }\end{array}$ & 126103 \\
\hline Piccolo & rabbit & 1 to 200 & $\begin{array}{l}\text { Synaptic } \\
\text { systems }\end{array}$ & 142003 \\
\hline Mover & rabbit & 1 to 500 & $\begin{array}{l}\text { Synaptic } \\
\text { systems }\end{array}$ & 248003 \\
\hline
\end{tabular}


Materials

\begin{tabular}{|c|c|c|c|c|}
\hline & & & & \\
\hline TGN38 & mouse & 1 to 500 & $\begin{array}{l}\text { BD- } \\
\text { Transduction } \\
\text { Laboratories }\end{array}$ & 610899 \\
\hline TGN38 & rabbit & 1 to 500 & $\begin{array}{l}\text { BD- } \\
\text { Transduction } \\
\text { Laboratories }\end{array}$ & 610898 \\
\hline GM130 & mouse & 1 to 500 & $\begin{array}{l}\text { Gift from Dr. } \\
\text { C. Dean }\end{array}$ & \\
\hline Syntaxin 6 & mouse & 1 to 300 & Abcam & 12370 \\
\hline Giantin & mouse & 1 to 1000 & Abcam & 3726 \\
\hline Giantin & rabbit & 1 to 1000 & Abcam & 24586 \\
\hline Vamp4 & rabbit & 1 to 1000 & $\begin{array}{l}\text { Synaptic } \\
\text { systems }\end{array}$ & 136002 \\
\hline GFAP & mouse & 1 to 1000 & $\begin{array}{l}\text { Synaptic } \\
\text { systems }\end{array}$ & 173011 \\
\hline Map2 & chicken & 1 to 6000 & $\begin{array}{l}\text { Synaptic } \\
\text { systems }\end{array}$ & 188006 \\
\hline Smi312 & mouse & 1 to 2000 & Covance & SMI-321R \\
\hline Calreticulin & rabbit & 1 to 1000 & Abcam & 2907 \\
\hline Ankyrin G & mouse & 1 to 400 & $\begin{array}{l}\text { Gift from } \\
\text { Dr. C. Dean }\end{array}$ & \\
\hline BDNF & chicken & 1 to 400 & Promega & G164A \\
\hline Rab3a & mouse & 1 to 300 & $\begin{array}{l}\text { Synaptic } \\
\text { systems }\end{array}$ & 107111 \\
\hline GFP & mouse & 1 to 3000 & Abcam & 1218 \\
\hline GFP & chicken & 1 to 3000 & Abcam & 13970 \\
\hline Synaptophysin & mouse & 1 to 1000 & Sigma Aldrich & $118 \mathrm{~K} 4828$ \\
\hline Synapsin $1 / 2$ & mouse & 1 to 500 & $\begin{array}{l}\text { Synaptic } \\
\text { systems }\end{array}$ & 106002 \\
\hline Homer1 & rabbit & 1 to 300 & $\begin{array}{l}\text { Synaptic } \\
\text { systems }\end{array}$ & 160003 \\
\hline SHANK2 & guniea pig & 1 to 1000 & $\begin{array}{l}\text { Synaptic } \\
\text { systems }\end{array}$ & 162202 \\
\hline PSD95 & mouse & 1 to 300 & Abcam & 99009 \\
\hline
\end{tabular}




\section{Secondary antibodies}

Table 3: A list of Alexa Flour dyes used for light microscopy and FLIM, Atto dyes and STAR dyes used for STED microscopy and RFP and GFP nanobodies used for STED microscopy and FLIM.

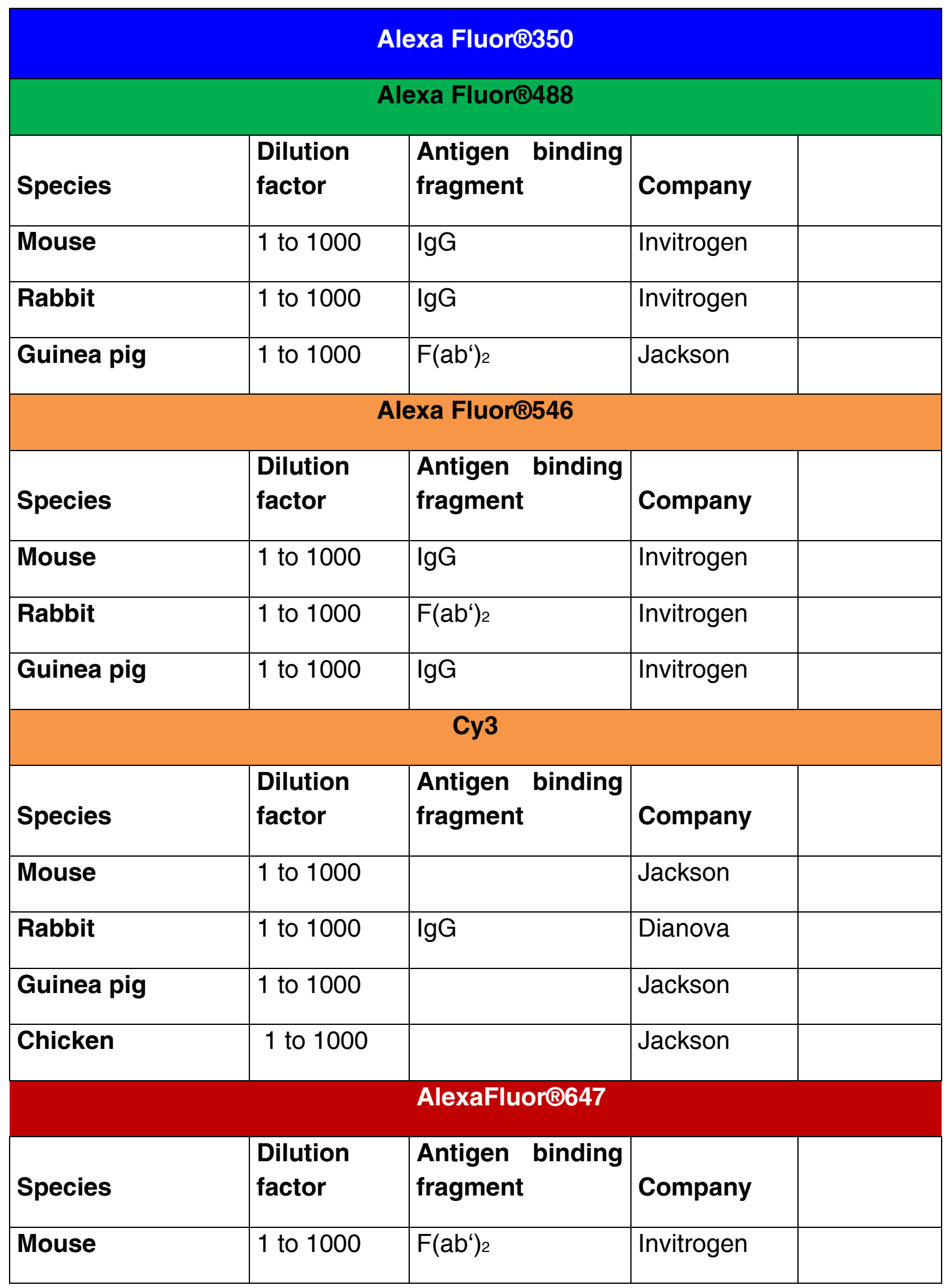




\begin{tabular}{|c|c|c|c|c|}
\hline Mouse & 1 to 1000 & & Jackson & \\
\hline Rabbit & 1 to 1000 & $\lg G$ & Mobitec & \\
\hline \multicolumn{5}{|c|}{ Cy5 } \\
\hline Species & $\begin{array}{l}\text { Dilution } \\
\text { factor }\end{array}$ & $\begin{array}{l}\text { Antigen binding } \\
\text { fragment }\end{array}$ & Company & \\
\hline Mouse & 1 to 1000 & $F\left(a b^{\prime}\right)_{2}$ & Jackson & \\
\hline Rabbit & 1 to 1000 & $\lg G$ & Invitrogen & \\
\hline Guinea pig & 1 to 1000 & $F\left(a b^{\prime}\right)_{2}$ & Jackson & \\
\hline \multicolumn{5}{|c|}{ Atto594 } \\
\hline $\begin{array}{l}\text { Göttfert } \\
\text { setup }\end{array}$ & $\begin{array}{l}\text { Dilution } \\
\text { factor }\end{array}$ & $\begin{array}{l}\text { Antigen } \quad \text { binding } \\
\text { fragment }\end{array}$ & Company & $\begin{array}{l}\text { Company } \\
\text { code }\end{array}$ \\
\hline Mouse & 1 to 100 & $F\left(a b^{\prime}\right)_{2}$ & ATTO-TEC & 130 \\
\hline Rabbit & 1 to 100 & $\lg G$ & ATTO-TEC & 184 \\
\hline Guinea pig & 1 to 100 & & ATTO-TEC & 501 \\
\hline \multicolumn{5}{|c|}{ RFP-BOOSTER } \\
\hline All STED setups & $\begin{array}{l}\text { Dilution } \\
\text { factor }\end{array}$ & $\begin{array}{l}\text { Antigen binding } \\
\text { fragment }\end{array}$ & Company & \\
\hline $\begin{array}{l}\text { RFP-Booster- } \\
\text { Atto594 }\end{array}$ & 1 to 300 & $\mathrm{~V}_{\mathrm{H}} \mathrm{H}$ & Chromotek & \\
\hline $\begin{array}{l}\text { RFP-Booster- } \\
\text { Atto647 }\end{array}$ & 1 to 300 & $\mathrm{~V}_{\mathrm{HH}}$ & Chromotek & \\
\hline \multicolumn{5}{|c|}{ Atto KK1212 } \\
\hline $\begin{array}{l}\text { Göttfert STED } \\
\text { setup }\end{array}$ & $\begin{array}{l}\text { Dilution } \\
\text { factor }\end{array}$ & $\begin{array}{l}\text { Antigen binding } \\
\text { fragment }\end{array}$ & Company & Code \\
\hline Mouse & 1 to 100 & $F\left(a b^{c}\right)_{2}$ & ATTO-TEC & 504 \\
\hline Rabbit & 1 to 100 & $\lg G$ & ATTO-TEC & 521 \\
\hline Guinea pig & 1 to 100 & & ATTO-TEC & 535 \\
\hline
\end{tabular}




\begin{tabular}{|c|c|c|c|c|}
\hline \multicolumn{5}{|c|}{ AttoK9445 } \\
\hline $\begin{array}{l}\text { Abberior STED } \\
\text { setup }\end{array}$ & $\begin{array}{l}\text { Dilution } \\
\text { factor }\end{array}$ & $\begin{array}{l}\text { Antigen binding } \\
\text { fragment }\end{array}$ & Company & Code \\
\hline Mouse & 1 to 100 & $F\left(a b^{\prime}\right)_{2}$ & ATTO-TEC & 259 \\
\hline \multicolumn{5}{|c|}{ STAR dyes } \\
\hline $\begin{array}{l}\text { Abberior STED } \\
\text { setup }\end{array}$ & $\begin{array}{l}\text { Dilution } \\
\text { factor }\end{array}$ & $\begin{array}{l}\text { Antigen binding } \\
\text { fragment }\end{array}$ & Company & Code \\
\hline Rabbit & 1 to 100 & $\lg G$ & Abbrerior & STAR 639 \\
\hline Mouse & 1 to 100 & $F\left(a b^{\prime}\right)_{2}$ & Abbrerior & STAR 638 \\
\hline Mouse & 1 to 100 & $F\left(a b^{\prime}\right)_{2}$ & Abbrerior & $\begin{array}{l}\text { STAR } \\
635 p\end{array}$ \\
\hline \multicolumn{5}{|c|}{ RFP-BOOSTER } \\
\hline All STED setups & $\begin{array}{l}\text { Dilution } \\
\text { factor }\end{array}$ & $\begin{array}{l}\text { Antigen binding } \\
\text { fragment }\end{array}$ & Company & \\
\hline $\begin{array}{l}\text { GFP-Booster- } \\
\text { Atto594 }\end{array}$ & 1 to 300 & $\mathrm{~V}_{\mathrm{H}} \mathrm{H}$ & Chromotek & \\
\hline $\begin{array}{l}\text { GFP-Booster- } \\
\text { Atto647 }\end{array}$ & 1 to 300 & $\mathrm{~V}_{\mathrm{H}} \mathrm{H}$ & Chromotek & \\
\hline
\end{tabular}

\subsubsection{List of DNA plasmids}

Table 4: A list of full length and mutant tagged DNA plasmids used in this study.

\begin{tabular}{|c|c|c|c|c|}
\hline DNA plasmid & Tag position & $\begin{array}{l}\text { Size } \\
\text { (amino } \\
\text { acids) }\end{array}$ & Resistance & $\begin{array}{l}\text { Obtained } \\
\text { from }\end{array}$ \\
\hline \multicolumn{5}{|l|}{$\begin{array}{l}\text { Bassoon } \\
\text { constructs }\end{array}$} \\
\hline $\begin{array}{l}\text { mRFP-Bassoon- } \\
\text { EGFP }\end{array}$ & $\begin{array}{ll}\text { Intramolecular } \mathrm{N}- \\
\text { Terminal } & \text { RFP } \\
\text { (97a.a) and } & \\
\text { C-Terminal } & \text { GFP } \\
\text { fusion tag } & \\
\end{array}$ & $1-3938$ & Ampicillin & $\begin{array}{l}\text { Prof.T. } \\
\text { Dresbach }\end{array}$ \\
\hline mRFP-Bassoon & $\begin{array}{l}\text { Intramolecular N } \\
\text { Terminal RFP } \\
\text { (97a.a) }\end{array}$ & $1-3938$ & Ampicillin & $\begin{array}{l}\text { Prof.T. } \\
\text { Dresbach }\end{array}$ \\
\hline
\end{tabular}


Materials

\begin{tabular}{|c|c|c|c|c|}
\hline mEGFP-Bassoon & $\begin{array}{l}\text { Intramolecular N } \\
\text { Terminal GFP } \\
\text { (97a.a) }\end{array}$ & $1-3938$ & Ampicillin & $\begin{array}{l}\text { Self } \\
\text { cloned }\end{array}$ \\
\hline Bassoon-mRFP & $\begin{array}{l}\text { C-Terminal GFP } \\
\text { fusion tag }\end{array}$ & $1-3938$ & Ampicillin & \begin{tabular}{|l|} 
Self \\
cloned
\end{tabular} \\
\hline Bassoon-mEGFP & $\begin{array}{l}\text { C-Terminal GFP } \\
\text { fusion tag }\end{array}$ & $1-3938$ & Ampicillin & $\begin{array}{l}\text { Prof.T. } \\
\text { Dresbach }\end{array}$ \\
\hline \multicolumn{5}{|l|}{ Bassoon mutants } \\
\hline $\begin{array}{l}\text { G2A mRFP- } \\
\text { Bassoon-EGFP } \\
\text { Mutant }\end{array}$ & \begin{tabular}{ll}
\multicolumn{2}{l}{ Intramolecular N- } \\
Terminal RFP \\
(97a.a) and \\
C-Terminal GFP \\
fusion tag
\end{tabular} & $1-3938$ & Ampicillin & $\begin{array}{l}\text { Prof. } \mathrm{T} . \\
\text { Dresbach }\end{array}$ \\
\hline EGFP-95-Bassoon & $\begin{array}{l}\mathrm{N} \text {-Terminal GFP } \\
\text { fusion tag }\end{array}$ & $95-3938$ & Ampicillin & $\begin{array}{l}\text { Prof. } \mathrm{T} . \\
\text { Dresbach }\end{array}$ \\
\hline $\begin{array}{l}\text { EGFP-Rbb26- } \\
\text { Bassoon }\end{array}$ & $\begin{array}{l}\mathrm{N} \text {-Terminal GFP } \\
\text { fusion tag }\end{array}$ & $2088-2563$ & Ampicillin & $\begin{array}{l}\text { Prof. } \mathrm{T} \text {. } \\
\text { Dresbach }\end{array}$ \\
\hline \multicolumn{5}{|l|}{ Other constructs } \\
\hline pEGFP-Munc13-1 & $\begin{array}{l}\mathrm{N} \text {-Terminal GFP } \\
\text { fusion tag }\end{array}$ & $1-1735$ & Kanamycin & $\begin{array}{ll}\text { Prof. } & \mathrm{N} . \\
\text { Brose }\end{array}$ \\
\hline $\begin{array}{l}\text { CFP-ß-1,4glycosyl } \\
\text { transferase } 1\end{array}$ & $\begin{array}{l}\mathrm{N} \text {-Terminal GFP } \\
\text { fusion tag }\end{array}$ & $0-81$ & Ampicillin & $\begin{array}{l}\text { Prof. } \mathrm{T} . \\
\text { Dresbach }\end{array}$ \\
\hline
\end{tabular}

\subsubsection{Bsn-/- knockout mouse}

The homozygous Bsn-/- knockout mice were generated by gene trapping introns 1 and 2 of Bassoon by VICTR 48 (gene trapping vector). The gene-trapped Bassoon vector was obtained from the Omnibank ES cell line OST486029 by Lexicon Pharmaceuticals, Inc. (The Woodlands, TX) ${ }^{42}$. Bsn-/- knockout mice were bred at the Leibniz Institute for Neurobiology in Magdeburg, Germany and were supplied as banker cultures by the Fejtova and Gundelfinger laboratories.

\subsubsection{Microscopes}

\section{Inverted Epifluoresence microscope}

Slides were visualized using an inverted Zeiss fluorescence microscope (Observer. Z1) with a Photometrics CoolSnap HQ2 camera at a magnification of 40X and 63X. The following filters from AHF were used: F46-000 for DAPI, F46002 for GFP and Alexa 488, F46-004 for Atto594 and Alexa 546 dyes and F46006 for AttoKK1212 and Alexa 647. Exposure times of 500ns for F46-002 and F46-004 filters and 1000ns for the F46-006 filter were applied. 


\section{Confocal Microscope}

Images were acquired on a Leica TCS SP2 laser-scanning microscope (Lieca Microsystems, CMS, Germany) with a $63 x / 1.4 N A$ oil-immersion objective. The $488 \mathrm{~nm}$-Argon laser and the He-Ne laser, for the $561 \mathrm{~nm}$ and $633 \mathrm{~nm}$ wavelengths, were used to excite respective Alexafluor types. All images were acquired with identical settings of 1 airy-pinhole size and a $500 \mathrm{~Hz}$ scan speed. These images were $1024^{\star} 1024$ pixels, imaged after 4 frame averages, at zoom factors of $1 \mathrm{X}$ (for the over view images) and $6 \mathrm{X}$ that possessed a pixel size of $230 \mathrm{~nm}$ and $38 \mathrm{~nm}$, respectively.

\section{STED microscopes}

\section{Bückers setup}

Figure 4, Figure 5, Figure 6, and Figure 8 were generated on a custom-built twocolor STED microscope ${ }^{88}$. Two excitation and two STED lazer beams, all supported by a single supercontinuum lazer source (SC-450-PP-HE, Fianium), were combined in the setup. Prism monochromators were used to set stringent excitation wavelength selections for Atto594 and Atto647 (at $570 \pm 2 \mathrm{~nm}$ and $650 \pm 2 \mathrm{~nm}$, respectively) and the STED wavelengths of $720 \pm 10 \mathrm{~nm}$. Dichroic mirrors were used to generate two beam pairs by combining the two excitation and two STED beams, which were coupled to a beam splitter and a 100x/1.400.7 oil objective lens (PL APO, Leica Microsystems, Wetzlar, Germany). The STED donut-shaped foci, that has a zero intensity minimum at its center, was generated by two vortex phase plates (RPC Photonics, Rochester, NY, USA.) and a superachromatic quarterwave plate $(600-2700 \mathrm{~nm}$, B. Halle $\mathrm{GmbH}$, Berlin, Germany) placed behind the 100x objective lens in the STED beam path. Two separated confocal detection units were used to detect excitation for Atto590 at $620 \pm 20 \mathrm{~nm}$ and Atto647N at $670 \pm 15 \mathrm{~nm}$ and both channels were scanned simultaneously in an interleaved fashion by applying a pulsed acquisition scheme.

\section{Göttfert setup}

STED images in Figures 15-Figure 19, Figure 22 and Figure 23, were acquired using a custom-built two-color STED microscope that is based on a confocal microscope that includes a 1.4 NA 100x objective (PL APO HCX 100x 1.4-0.7 Oil, Leica Microsystems, Wetzlar), and a 775nm STED laser (ELP-5-775-DG, IPG Photonics Corporation, Oxford, MA, USA). The dyes were excited at wavelengths of $470 \mathrm{~nm}, 595 \mathrm{~nm}$ and $640 \mathrm{~nm}$ while the fluorescence was detected with avalanche photo diodes from 500-550nm, 600-640 nm and 660-720nm respectively. Epifluorescence images were acquired using a LED illumination source, a monochrome filter and camera (DMK41 AU02, The Imaging Source). For the overview images the LED illumination source was manually installed every session for one color, wherein a 590nm LED was installed with the 700/60 
fluorescence filter and 640RDC dichroic filter or a 490nm LED was installed (upon requirement) with the 450/60 fluorescence filter, in the camera path. The 775 STED beam is circularly polarized by being passed through a $0-360^{\circ}$ vortex linear phase ramp and a $\lambda / 4$ plate to generate a focal beam "doughnut" that has zero intensity in the center. The doughnut-shaped STED beam only allows fluorescence around the zero intensity point. The images were obtained at using 300-700 mW STED power, $4 \mu \mathrm{W}$ excitation power, dwell time of 30-100 microseconds and a pixel size of $10 \mathrm{~nm}$. The resolution regularly obtained for Atto594 antibodies was $30-45 \mathrm{~nm}$ and for AttoKK1212 antibodies was 20-35nm (at $300 \mathrm{~mW}$ and $700 \mathrm{~mW}$ STED-power, respectively).

\section{Abberior setup}

The images shown in Figure 24 and Figure 25, were taken on Abbrerior instruments QuadSCAN 2 color STED microscope. The setup possesses a pulsed $775 \mathrm{~nm}$ STED lazer and two pulsed excitation lazer sources at $594 \mathrm{~nm}$ and $640 \mathrm{~nm}$ integrated into a Olympus IX83 microscope that is equipped with $100 \times 1.4$ NA objective, a 4-color LED illumination source and a monochrome wide field. The Abberior QUAD scanner applies four galvo mirrors that provide four independent degrees of freedom and angle settings to allow for faster scan speeds and the images are detected with a gated avalanche photodiode (APD). A pixel size of $20 \mathrm{~nm}$, dwell time of $3 \mathrm{~ms}$ and line accumulation of 3 were applied.

\section{FLIM microscope}

FLIM imaging was performed on an FV1000 lazer-scanning microscope with a UPlanS-Apo 60X 1.35-NA objective (Olympus) and a pulsed laser (Picoquant) running at $470 \mathrm{~nm}$, at a $40 \mathrm{MHz}$ repetition rate. Over view images were acquired at 2-5\% LED power at the wavelength range of $505-545 \mathrm{~nm}$ for GFP or Alexa Fluor $\AA 488,570-625 \mathrm{~nm}$ for RFP and Atto594 and 640-670nm for Alexa Fluor®647. Fluorescence lifetime was recorded with an avalanche photodiode (Micro Photon DevicesPDM) coupled to a Picoharp-300 (Picoquant). GFP lifetimes were recoded using a $520 \pm 15 \mathrm{~nm}$ filter in front of the FLIM detector. In order to avoid detector overload and to ensure stable and long lifetime imaging at minimum excitation intensity, images were recorded at 200Kcounts over 10 minutes. The raw PT3 images were converted into *ics format using PT32ICS ${ }^{89}$ (Geert van den Bogaart). Subsequently a mono-exponential phasor plot analysis was performed using TRI2 2.8.5.1 (Paul Barber,Gray Institute, Oxford, Uk) to obtain lifetime images. 


\subsection{Methods:}

\subsubsection{Primary culture of rat and mouse hippocampi}

\section{Rat primary culture}

Primary cultures of hippocampal neurons from E19 rats were prepared according to a modified Goslin and Banker protocol ${ }^{90}$ (1998). These neurons were trypsinized in Hanks' Balanced Salts Solution (HBSS) (Life Technologies), containing $0.25 \%$ trypsin (Invitrogen) for 20 minutes at $37^{\circ} \mathrm{C}$. These cells were then washed and dissociated in Dulbecco's modified Eagle's medium (DMEM) (Life technologies), containing 10\% fetal calf serum (FCS) (Biochrom). 50,000 cells per well in 24-well dishes were plated on Poly-D-Lysin (Sigma Aldrich) coated coverslips. The neurons were plated and incubated in the plating medium, which contained Penicillin and Streptomycin (Pen-Strep 100U/ml from Life technologies) and 2mM L-glutamine (Invitrogen). 24 hours after incubation, the plating medium was substituted with Neurobasal medium (Life technologies), containing 2\% B27 (Life technologies), 2\% antibiotics and 0.5\% L-Glutamine. The neurons were then left to incubate at $37^{\circ} \mathrm{C}$ and $5 \% \mathrm{CO}_{2}$.

\section{Mouse primary culture (for Bsn-/- knockout mice and $\mathrm{Bsn}^{+} /^{+}$ littermate controls)}

P0-P1 wild type mice cortexes were dissected to generate the feeder layer of a banker culture. Hemispheres of cortexes with their meneges removed were chopped up in $4.5 \mathrm{ml}$ of HBSS and incubated for 15 minutes at $37^{\circ} \mathrm{C}$ in $2.5 \%$ trypsin (without EDTA). These pieces were then washed in HBSS and dissociated in glia medium that consisted of $90 \%$ plating medium and $10 \%$ DNAse (Invitrogen). Both hemispheres of one brain were dissociated in $1 \mathrm{ml}$ glia medium and plated in $10 \mathrm{ml}$ of plating medium that was identical to rat primary culture plating medium. The medium was changed every $4-5$ days, and the confluent glia were trypsinated, washed in HBSS, and $5 \mathrm{ml}$ of the glia were plated on a $6 \mathrm{~cm}$ dish.

Two P1 Bsn-/- knockout mice and two wild-type $\mathrm{Bsn}^{+/+}$littermates were prepped into dissociated primary hippocampal neurons following the same protocol as was used for rat primary culture. $100 \mathrm{ml}$ of 5000 hippocampal cells were plated on coated $18 \mathrm{~mm}$ round glass cover slips. These coverslips were first incubated for 1 hour at $37^{\circ} \mathrm{C}$ and $5 \% \mathrm{CO} 2$ and then transferred, neurons facing down, on to dishes containing the feeder layer of glia and $5 \mathrm{ml}$ of culturing medium $(94 \%$ Neurobasal, 2\% Glutamax (Invitrogen), 2\% B27, 1\% NaPyr (0.1M), 1\% Pen/Strep $(0.1 \mathrm{M})$. These coverslips were left to grow at $37^{\circ} \mathrm{C}$ and $5 \% \mathrm{CO} 2$ and were treated with $2 \mu \mathrm{l}$ Ara-C (Sigma) on day in vitro 1 (DIV1) and DIV3 to prevent glia 
overgrowth and were fed once a week with an exchange of $1 \mathrm{ml}$ of fresh culturing medium to maintain optimal growth of the culture.

\subsubsection{Cloning of full length Bassoon constructs}

Full length Bassoon constructs mRFP-Bassoon-mEGFP, mRFP-Bassoon and mutant G2A-mRFPBassoon-mEGFP were provided in a ampicillin resistant $\mathrm{PCS}^{+}$vector backbone and were designed to possess an intramolecular RFP at the 97 amino acid and/or a GFP fused to the $C$ terminus of Bassoon such that the stop codon of the sequence was abolished. The constructs were designed by Prof. T Dresbach and generated by Clonetech.

\section{Molecular biology techniques}

To clone mEGFP-Bassoon, Bassoon-mEGFP and Bassoon-mRFP constructs a range of basic molecular biological protocols were employed and are described here.

\section{Digestion Protocol}

$1 \mu \mathrm{l}$ of HindlII (Fermentas fast digest enzyme, Thermo Scientific)

$1 \mu \mathrm{g}$ of DNA Plasmid

$2 \mu \mathrm{l}$ 10X Green fast digest buffer (Thermo Scientific)

$x \mu \mathrm{l}$ of $\mathrm{H}_{2} \mathrm{O}$ in a total volume of $20 \mu \mathrm{l}$ for 30 minutes at $37^{\circ} \mathrm{C}$

\section{Ligation Protocol}

25ng of linearized DNA plasmid

125ng of DNA insert

$1 \mu \mathrm{l}$ of 10X T4 DNA-ligase ligation buffer

$0.5 \mu$ of T4 DNA-ligase

$x \mu \mathrm{l}$ of nuclease free $\mathrm{H}_{2} \mathrm{O}$ in a total volume of $10 \mu \mathrm{l}$.

This mixture was incubated overnight at $16^{\circ} \mathrm{C}$ and the ligation mixture was heat inactivated at $65^{\circ} \mathrm{C}$ for 10 minutes.

\section{Transformation Protocol}

A $100 \mu \mathrm{l}$ of XL-1 Blue competent cells (thawed on ice) are placed in a pre-chilled tube. $10-50$ ng of DNA plasmid or $1-5 \mu$ of a ligation mix is added by swirling the tube gently on ice and left to incubate on ice for 30 minutes. The tube is then heat pulsed on $42^{\circ} \mathrm{C}$ block for 45 seconds and directly incubated for 2 minutes on ice. $900 \mu \mathrm{l}$ pre-warmed SOC medium is added to the cells and grown on a $37^{\circ} \mathrm{C}$ shaker for 1 hour at $225-250 \mathrm{rpm} .500 \mu \mathrm{l}$ of this transformation mixture is plated on a pre-warmed LB-ampicillin and incubated for 17 hours at $37^{\circ} \mathrm{C}$.

\section{Sequencing Protocol (GATC Biotech)}

100ng of DNA plasmid

$5 \mu \mathrm{M}(5 \mathrm{pmol} / \mu \mathrm{l})$ of primer

$x \mu l$ of nuclease free $\mathrm{H}_{2} \mathrm{O}$ in a total volume of $10 \mu \mathrm{l}$. 
This mixture was sent to GATC Biotech in a $1.5 \mathrm{ml}$ eppendorf tube with a prepaid barcode. The sequences of the sequencing primers used for the cloning of the single labeled full length Bassoon constructs are listed in Table 5.

Table 5: Sequencing primers for full length Bassoon.

\begin{tabular}{|l|l|l|l|}
\hline Primer & Primer Sequence & $\begin{array}{l}\text { Temperature } \\
\left({ }^{\circ} \mathbf{C}\right)\end{array}$ & Length \\
\hline Bsn_FW_1 & CTAATGGGAGGTCTATATAAG & 55.4 & 21 \\
\hline Bsn_FW_2 & $\begin{array}{l}\text { AGCACTAGCTGGCGGCGGAC } \\
\text { A }\end{array}$ & 67.3 & 21 \\
\hline mRFP-FW-3a & GTAATGCAGAAGAAGACCATG & 57.5 & 21 \\
\hline mRFP-Rev-3b & CATGGTCTTCTTCTGCATTAC & 57.5 & 21 \\
\hline Bsn_FW_3c & GGGCTTCAAGTGGGAGCG & 64.238 & 18 \\
\hline Bsn_FW_4 & GGGCCAGGAGGAGACAGACG & 67.3 & 21 \\
\hline Bsn_FW_5 & GCTCCAAACCGGCAGCCAAAG & 65.3 & 21 \\
\hline
\end{tabular}

\section{mEGFP-Bassoon Cloning}

The mRFP-Bassoon construct was modified to mEGFP-Bassoon by inserting a mEGFP at the intramolecular 97 amino acid position. Four micrograms of mRFPBassoon, in $80 \mu \mathrm{l}$ volume were digested using Hindlll. This blunt digestion was dephosphorylated at its $5^{\prime}$ and $3^{\prime}$ ends with $1 \mu \mathrm{l}$ of CIP (England Biolabs) for 30 more minutes. Simultaneously 3 tubes containing $1 \mu \mathrm{g}$ each of HindlllmEGFP-HindIII-PUC vector (Clonetech), were also digested in a $20 \mu \mathrm{l}$ volume each, with Hindlll. These digested products were run for 1.5 hours on a $1 \%$ agarose gel (made by boiling $1 \%$ agarose in $20 \mathrm{ml}$ of $1 \mathrm{X}$ TAE buffer and $3 \mu \mathrm{l}$ of Midori green) alongside a $1 \mathrm{~kb}$ DNA ladder (Thermo Scientific). A 16000bp band for Bassoon (without mRFP) and three separate 750bp bands of HindllI-mEGFPHindllI were cut out and eluted using the PCR and Gel Purification Kit (Promega). Subsequently, the purified products were ligated in 1 volume of linearized Bassoon plasmid : 5 volumes HindIII-mEGFP-HindIII insert.

XL-1 Blue competent cells (Stratagene) were transformed using $1-5 \mu \mathrm{l}$ of the ligation mix and grown on LB Ampicillin plates at $37^{\circ} \mathrm{C}$ for 16 hours. 20 colonies from this plate were picked and grown in $3 \mathrm{ml}$ of warm LB-ampicillin broth and grown for 17 hours. A DNA mini prep was performed from $2 \mathrm{ml}$ of the bacterial culture of all 20 colonies using the Qiagen Mini Prep DNA Isolation Kit and following the manufacturer's protocol, and $1 \mathrm{ml}$ of each colony culture was saved at $4^{\circ} \mathrm{C}$. The DNA isolated from each colony was sequenced using primers Bsn_FW_1, Bsn_FW_3c, mRFP-Rev-3b, and Bsn_FW_5 (Sequences provided in Table 5 above). The saved culture of one correctly cloned colony was used to 
inoculate $200 \mathrm{ml}$ of LB-ampicillin broth, grown for 17 hours at $37^{\circ} \mathrm{C}$ and its DNA isolated using the Qiagen endofree Maxi Prep DNA Isolation Kit according to the manufacturer's protocol. A second round of sequencing was performed with the same primers to double check construct created. The mEGFP-Bassoon was also checked for its DNA concentration and verified for its purity (using a spectrophotometer) and finally stored at $-20^{\circ} \mathrm{C}$ to be used for transfections.

\section{Bassoon-mRFP Cloning}

Bassoon-mRFP was cloned by replacing mEGFP of the Bassoon-mEGFP construct with an mRFP tag from the Mlu-mRFP-Spel-PUC vector (Clonetech). Bassoon-mEGFP $(4 \mu \mathrm{g})$ was digested in $40 \mu \mathrm{l}$ with Mlu and Spel and dephosphorylated using CIP as is described above. Three tubes each with $1 \mu \mathrm{g}$ of mRFP-PUC were also digested similarly with Mlu and Spel. The digestion products, the linearized Bassoon vector (expected16000bp band) and three wells of Mlu-RFP-Spel (700bp expected bands), were run on an agarose gel, their respective expected band sizes cut out, eluted with the gel extraction kit, and ligated with a 1:5 ratio of Mlu-Bassoon-Spel vector: Mlu-mRFP-Spel. This ligation mix is on the following day transformed into XL1 Blue competent cell, grown on LB-ampicillin plates and 20 colonies of the plate were grown, mini prepped and sequenced. Sequencing primers (Table 5) Bsn_FW_1, Bsn_FW_3c, Bsn_FW_4 and Bsn_FW_5 were used to confirm colonies that were successfully cloned. One such colony was regrown, maxi prepped, verified for concentration, purity and sequence and store at $20^{\circ} \mathrm{C}$ for future transfections.

\subsubsection{Transfection methods}

\section{Early calcium phosphate transfection (transfection: DIV 2-4 fixation: DIV 14-24)}

This protocol is the relevant choice for generating neurons tagged for a protein of desire that need to be studied post DIV15. Young DIV3 neurons are first substituted and incubated for a minimum of 10 minutes with $500 \mathrm{ml}$ of $37^{\circ} \mathrm{C}$ Optimem and their conditioned medium saved in a sterile container. To prepare a DNA-calcium-phosphate precipitate mix, $15 \mathrm{ml}$ of transfection buffer $(274 \mathrm{mM}$ $\mathrm{NaCl}, 10 \mathrm{mM} \mathrm{KCl}, 1.4 \mathrm{mM} \mathrm{Na2HPO} 4,15 \mathrm{mM}$ Glucose, 42 mM HEPES, pH 7.06) were added in a dropwise manner to a $15 \mathrm{ml} /$ well of solution containing $1.87 \mathrm{ml}$ of $2 \mathrm{M} \mathrm{CaCl}_{2}$ and $1 \mathrm{mg}$ DNA plasmid. This mixture was left to incubate for 20 minutes at room temperature, and $30 \mathrm{ml}$ DNA-calcium-phosphate precipitate was added to each coverslip and left to incubate for 60 minutes. The neurons were then washed three times with $1 \mathrm{ml}$ of $37^{\circ}$ pre-warmed Neurobasal, followed by two $750 \mathrm{ml}$ exchanges. Finally, the neurons were reinstated into their original conditioned medium and returned to the incubator. This protocol has a lower yield 
of transfected neurons compared to the late Lipofectamine protocol, but the transfected neurons have higher survival rates into late developmental stages.

\section{Early Lipofectamine transfection (transfection: DIV 2-6 fixation: within 24 hours)}

To tag proteins during Golgi association and during early transport in the neurons, DIV5/6 neurons were transfected using the Lipofectamine method. Neurons plated on $12 \mathrm{~mm}$ coverslips in 24-well plates had their conditioned medium exchanged and replaced with $500 \mathrm{ml} 37^{\circ} \mathrm{C}$ Neurobasal medium containing $2 \%$ B-27 and $1 \%$ of $2 \mathrm{mM} \mathrm{L-Glutamine;} \mathrm{they} \mathrm{were} \mathrm{then} \mathrm{incubated} \mathrm{for} \mathrm{a}$ minimum of 10 minutes. $1 \mu \mathrm{g}$ of plasmid DNA in $25 \mathrm{ml}$ Optimem (Life Technologies) was mixed with $1 \mathrm{ml}$ of Lipofectamine 2000 (Invitrogen) in $25 \mathrm{ml}$ of Optimem. This mix was incubated at room temperature for $20 \mathrm{~min}$ after which 50 $\mathrm{ml}$ of it was added to each well. After 1.25 hours of incubation at $37^{\circ} \mathrm{C}$ and $5 \%$ $\mathrm{CO} 2$, the transfected coverslips were washed three times with $37^{\circ} \mathrm{C}$ Neurobasal medium and reinstated in their conditioned medium. These neurons were checked after $24 \mathrm{hrs}$ for health and transfection efficiency by light microscopy and were fixed for 20 minutes in cold $4 \%$ paraformaldehyde solution before immunocytochemistry was performed.

\section{Early mouse sandwich-culture Lipofectamine transfection (transfection: DIV 2-4 fixation: within 24 hours)}

The $18 \mathrm{~mm}$ coverslips plated with Bsn-/- knockout mice and wild-type $\mathrm{Bsn}^{++}$mice were moved to a 12-well plate containing $600 \mathrm{ml}$ warm Optimem and left to acclimatize in the incubator at $37^{\circ} \mathrm{C}$ and $5 \% \mathrm{CO}_{2}$. In addition, a tube containing $50 \mathrm{ml}$ of Optimem/well and $1.5 \mu \mathrm{g}$ of DNA plasmid/well, and another containing $50 \mathrm{ml}$ of Optimem/well and $2.25 \mathrm{ml}$ of Lipofectamine 2000 / well, were mixed and left to incubate for 20-30 minutes at room temperature. This DNALipofectamine mix was then, without mixing, applied in $100 \mathrm{ml}$ volumes to the equally distributed over each coverslip and left to incubate for 3 to 4 hours. The coverslips were subsequently transferred back directly into their original $6 \mathrm{~cm}$ dish, which possessed their original condition medium and the feeder layer. These neurons were left to grow for 24 hours at $37^{\circ} \mathrm{C}$ and $5 \% \mathrm{CO}_{2}$ and were then fixed in a 12-well plate containing $600 \mathrm{ml}$ per well of cold $4 \%$ paraformaldehyde solution for 20 minutes, in preparation for the immunocytochemistry protocol.

\section{Late Lipofectamine transfection (transfection: DIV 10 fixation: DIV 13-14)}

DIV10 primary hippocampal neurons on $12 \mathrm{~mm}$ coverslips had their conditioned medium exchanged with $400 \mathrm{ml}$ of $37^{\circ} \mathrm{C}$ Neurobasal medium with $2 \% \mathrm{~B}-27$ and 2 $\mathrm{mM} \mathrm{L-Glutamine} \mathrm{and} \mathrm{incubated} \mathrm{for} \mathrm{a} \mathrm{minimum} \mathrm{of} 10$ minutes. Meanwhile a DNA 
transfection mix was prepared consisting of $0.75 \mu \mathrm{g}$ of plasmid DNA in $50 \mathrm{ml}$ Optimem and was mixed with $1 \mathrm{ml}$ of Lipofectamine 2000 (Invitrogen) in $50 \mathrm{ml}$ of Optimem. This DNA-Lipofectamine mix was incubated at room temperature for 20-30 minutes and $100 \mathrm{ml}$ of this DNA mix was applied onto the coverslips and left to incubate for 3 hours. These coverslips were then washed once with prewarmed Neurobasal medium and reinstated into their original conditioned medium and returned to grow in the incubator. These neurons were allowed to express the DNA plasmids for no longer than 5 days as after this point overexpression artifacts were readily observed. This protocol yields higher transfection efficiencies compared to the calcium phosphate protocol and is applicable for experiments in which the neurons do not have to survive for longer than DIV15.

\subsubsection{Immunocytochemistry}

The hippocampal neurons were fixed using cold $4 \%$ paraformaldehyde for 20 minutes and washed three times with 1X PBS. To reduce un-specificity of antibody binding, a primary block buffer (10\% FBS, $5 \%$ sucrose, $2 \%$ albumin, $0.3 \%$ Triton X-100 in $1 \times$ PBS) was applied for 20 minutes. The primary antibodies diluted in the primary block solution were left overnight at $4^{\circ} \mathrm{C}$. All secondary antibodies were diluted in secondary antibody-buffer $(0.3 \%$ Triton X-100, 5\% sucrose and $2 \%$ albumin in $1 \times$ PBS) and incubated for 1 hour at room temperature, protected from light. Coverslips were subsequently washed three times with 1X PBS and once with distilled water before being mounted in $8 \mathrm{ml}$ DABCO-mowiol (Calbiochem) and left to dry overnight.

\subsubsection{Image processing}

\section{Epifluorescence images}

The images were opened in Image $\mathrm{J}$ software $(\mathrm{NIH})$ and merged from single channel, 8-bit, black and white images into RGB merged tiffs. Depending on need, they were adjusted for brightness and contrast, calculated and stamped with a suitable sized scale bar and saved as TIFF files for further analysis with Metamorph and Imaris MeasurementPro software.

\section{STED images}

Images acquired on all STED setups used were processed using the Richardson-Lucy deconvolution function integrated into Imspector Software ${ }^{91}$ (Max Planck Innovation). The point spread function for deconvolution was generated by using a 2D Lorentz function with its full width at half maximum (FWHM) fitted to the resolution estimate of each individual image. These images 
were then analyzed using Imaris MeasurementPro. Images for figures were adjusted for brightness and contrast with Image $\mathrm{J}$ software $(\mathrm{NIH})$.

\section{FLIM images}

Lifetime images were obtained in a PT3 format and converted using the PT32ICS software (Geert van den Bogaart). These images were then subjected to a monoexponential phasor plot analysis was performed using TRI2 2.8.5.1 software (Paul Barber, Gray Institute, Oxford, UK). The pixel density function values were extracted for each analyzed lifetime image. The Alexa 488 lifetime range of 1.52.0 nanoseconds (for Figure 20), the GFP lifetime range (boosted by Alexa 488) of 2.2-3.0 nanoseconds (for Figure 26), and the GFP lifetime range of 1.5-2.5 nanoseconds (for Figure 27) were applied to all images in their respective experimental and their corresponding values were exported into Microsoft Excel. FRET images were generated by applying an inverted, 16-color look up table to the lifetime images, in their respective lifetime ranges, which were exported as TIFF files from TR12 and converted into a RGB file or HSB stack with Image J. Layer B of the HSB stack was replaced by the corresponding Alexa 488 intensity image to generated the FRET or Lifetime overlay images. All raw lifetime values were normalized to unity and fitted to a Gaussian distbribution in GraphPad Prism. These values were then converted to FRET efficiencies using the formula below and plotted in GraphPad.

$$
E=1-\frac{\tau_{D A}}{\tau_{D}}
$$

$\mathrm{E}=\mathrm{FRET}$ Efficiency; $\tau \mathrm{DA}=$ Donor Lifetimes ${ }^{*}$ Acceptor Lifetimes (ns), $\tau \mathrm{DA}=$ Donor Lifetimes (ns).

The mean and SEM for each curve was calculated and an unpaired Student's $t$ test was performed, for Figure 24 and Figure 25, to statistically test the difference in lifetimes of the data sets.

\subsubsection{Analysis software and application}

\section{MetaMorph Colocalization}

Merged multi-channel 40X light microscopy images were analyzed using MetaMorph Offline Version 7.7.0.0 (Molecular Devices, Inc.). A threshold is set for each channel followed by the generation of a mask for all channels, in three areas of size 25 pixels long (representing $2 \mu \mathrm{m}$ in the sample) and 4 pixels wide, per image. These area masks were then overlaid in the arithmetic tool and divided to generate a third mask containing only the population of signals in the 
mask that do colocalize. The amount of bassoon colocalized is a represented as a percentage of the bassoon colocalized population divided by total bassoon population.

\section{Imaris MeasurementPro 8.1(Bitplane AG.) software}

Merged TIFF light microscopy images or STED images were analyzed using Imaris for range of analyses such as, the probability of colocalization (Pearson's correlation coefficient), amount of colocalization, signal sizes and their population, and distribution of signals.

For Figure 4-Figure 5, Figure 15-Figure 19, Figure 22-Figure 24, a free-hand drawn mask was drawn to exclude all signals in the image that were in the nucleus, outside the cell soma, or in the axons. To factor out the effect of the varying number of signals counted per size of hand-drawn mask in each image, the area in $\mu \mathrm{m} 3$ of the mask used, was divided by the signals counted per image.

\section{Probability of colocalization}

Images with or without masks were all then run through the ImarisColoc module that is integrated with a Costes P-Value approximation ${ }^{92}$ plugin, to generate automated thresholds for all the channels of all the images in a set. Also this module is integrated with Image J plugin: just another colocalization plugin that can calculate the colocalization Pearson's and Manders' correlation coefficient Constants in the image.

To ascertain the amount of colocalization, the signal sizes and distribution of the spot signals in the image, the Imaris Spots module was first used to generate objects for each spot of signal in the image, for all channels. These objects are generated upon applying the automated intensity threshold value calculated by the Costes P-Value approximation, signal diameter size range of $30-160 \mathrm{~nm}$ for STED images and $>200 \mathrm{~nm}$ for light microscopy images, an automated splitting of cluster signals (defined as $>120 \mathrm{~nm}$ for STED images and $>400 \mathrm{~nm}$ for light microscopy images) based on intensity profile plots of signals within the cluster, in each channel.

\section{Signal Sizes and their populations}

Imaris Spots calculates a range of statistical data for the spot objects generated for each channel. One such automatically generated type of data is the diameter size (determined by the FWHM of the PSF) of each spot signal. These data were collected in Excel for Figure 5 for all the images in the set, and the total number of spots in the size range of $30-60 \mathrm{~nm}, 60-90 \mathrm{~nm}$ and $90-130 \mathrm{~nm}$ were calculated and plotted using GraphPad Prism.

\section{Amount of colocalization}

The amount of colocalization was calculated using the spot objects generated for each channel and a MATLAB extension in the spots module called Spots 
colocalize, which calculates the colocalized population of all channels using the a distance threshold of $0-100 \mathrm{~nm}$ (for STED images) and 0-350nm (for light microscopy images) from the spot centers.

\section{Distribution of Signals}

To analyze the distribution of AZP to a Golgi marker, the distance transformation MATLAB extension94 from the Imaris XT module was used. This extension exchanges the voxel intensities data of all signals in the Golgi marker channel into spot coordinates data and creates a new channel with this data. This channel indicates the shortest distance to the object border of the Golgi marker spot. The AZP channel is overlaid over the Golgi marker distance transformation channel to reveal the shortest distances between all the AZP signals and the border coordinates of the all the Golgi marker signals. The shortest distance value for every AZP spot to a Golgi marker spot can as easily be exported to excel, as well as the total number of AZP signals within $0-100 \mathrm{~nm}$ or $101-100 \mathrm{~nm}$ distance ranges.

\section{GraphPad Prism 5.02}

All resulting data were analyzed and graphically represented using GraphPad Prism 5.02. Comparisons between groups were statistically tested, and the data in the graphs are presented as mean \pm SEM. Differences were considered significant $\left({ }^{*} p<0.05\right)$, strongly significant $\left({ }^{* *} p \leq 0.01\right)$ and extremely significant $\left.{ }^{* * *} p \leq 0.001\right)$. For Figure 3-Figure 8, Figure 10-Figure 13, and Figure 15Figure 19, a one-way annova test was performed with a post-hoc test of Tukey's multiple comparisons test (that compares the means of all columns). For every significant difference noted between the two relevant groups, an additional twotailed, unpaired Student's $t$ test with different variances was also performed to reveal the same significant difference. 
Results

\section{Chapter 3}

\section{Results}

This chapter details my results, in which I study the localization and ultrastructure of active zone proteins (AZPs) at various subcellular structures, on their journey from the soma of young developing neurons, to the presynaptic cytomatrix at the active zone (CAZ). A deeper understanding of how AZPs are oriented, organized, and transported, at various sites in the developing neuron, is required for unraveling the mechanisms at play during mammalian active zone assembly and CAZ maturation. To tackle these topics, I will here first study the ultrastructural localization of endogenous AZPs at Golgi substructures, in the soma, and during transport in the axons of young hippocampal neurons. I will then compare the endogenous AZP localizations to the localization of well-established recombinant AZP constructs, and will finally introduce, characterize, and use new tools made off the full-length Bassoon molecule. These second generation constructs have been optimized and used to study the orientation, organization, and the detailed localization of this AZP at the Golgi, the soma, on trafficking dense-core vesicles, and at synaptic sites. 


\subsection{Localization of endogenous AZPs at the Golgi apparatus}

Initial studies in the field have shown AZPs Bassoon and Piccolo to be localized in the somas of young neurons at Golgi substructures and transported to presynaptic sites on $80 \mathrm{~nm}$ dense-core vesicles known as Piccolo-Bassoon Transport Vesicles (PTVs) ${ }^{30,68,69}$. Other AZPs, namely Munc13-1, RIM1 $\alpha$, and ELKS2, were also isolated in light brain fraction immunoprecipitates containing PTVs $^{69,33}$. Golgi-derived AZP transport carriers, which are either preassembled $\mathrm{PTVs}^{93}$ or small clusters of clear- and dense-core vesicles carrying $\mathrm{AZPs}^{71}$, are believed to sufficiently transport the entire CAZ scaffold to the presynaptic membrane for the generation of a functional synapse.

\subsubsection{Localization of AZPs to Golgi sub-compartments with and without a $19^{\circ} \mathrm{C}$ block (confocal)}

The biogenesis of PTVs and other AZ transport carriers still remains unclear, though a recent study has shown Piccolo, Bassoon, and ELKS2 localized to the trans-Golgi sub-compartment, Munc13-1 localized to the cis-Golgi subcompartment and RIM $1 \alpha$ diffusely distributed through the neuronal processes of young neurons ${ }^{32}$. These results compliment my own observations but lacked a detailed description of how AZPs are distributed, sorted and localized at these Golgi substructures.

To explore the extent of localization of a Golgi associated AZP to its Golgi subcompartment, I co-immunostained young neurons for AZP markers Bassoon, Munc13-1 and Piccolo at the cis-Golgi and the trans-Golgi network markers, GM130 and TGN38, respectively. In order to determine proper localization of AZP to Golgi substructures with confocal microscopy, stainings were performed in neurons blocked for 45 minutes at $19^{\circ} \mathrm{C}$ to arrest AZP transport out of the Golgi. This generates enough AZP signal around a Golgi substructure for confocal imaging ${ }^{30}$ and allows me to directly compare my results to related results in the field, which were similarly processed.

All three AZP were imaged from the top of the soma, where the first cis-Golgi signals appear, to the bottom of the soma, closer to the coverslip, which ends with the last deeper trans-Golgi network signals. Bassoon and Piccolo present a higher visual degree of colocalization (observed as the high intensity yellow overlapping signals) to the TGN38 and are in best focus deeper in the $5 \mu \mathrm{m}$ Golgi stack imaged. Both the proteins are also partially localized to the GM130 signals and may not be well confined to a single Golgi sub-compartment as the $19^{\circ} \mathrm{C}$ block arrests a much higher number of AZP than the unblocked situation, around all Golgi substructures. An opposite localization pattern is observed for Munc13- 
1 , which is highly colocalized within the upper layers of the imaged stack, at the highest GM130 signals and has some, albeit lower, visual colocalization to the TGN38 marker (Figure 3).

Figure 3: Bassoon, Piccolo and Munc13-1 associate with specific Golgi sub-compartments. A-F, confocal images of DIV7 hippocampal neuron cell somas were immunostained with antibodies against Bassoon (A-B), Munc13-1 (C-D) and Piccolo (E-F) at cis- and trans-Golgi markers TGN38 and GM130, respectively. All images were taken after a 45 minute $19^{\circ}$ block before the paraformaldehyde fixation to arrest enough proteins to properly visualize their location. $5 \mu \mathrm{m}$ stacks of the soma were imaged with $200 \mathrm{~nm} Z$ slices. The $Z$ value represents the position in the stack. Scale bars $(\mathbf{A}-\mathbf{F}), 5 \mu \mathrm{m}$.

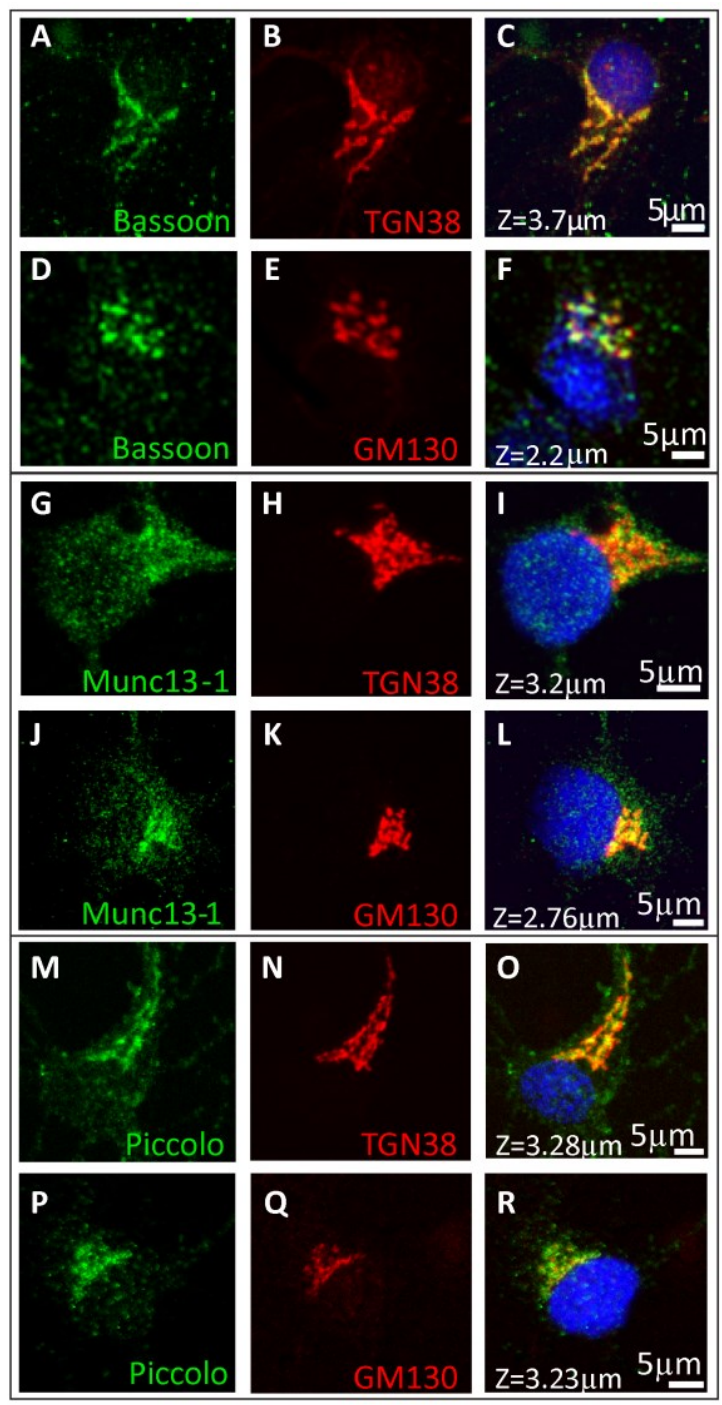

The localization of Bassoon and Piccolo to the TGN and Munc13-1 to the cisGolgi, is in congruence with previously reported associations of these proteins to Golgi substructures from our lab and others in the field ${ }^{30,32}$, and elude towards the presence of different AZP transport precursors - distinct not only in AZP composition but specific for the Golgi lamella they were derived from, which will ultimately influence their sorting in the soma, the pathway and mode of transport they take to the AZ. This study additionally reveals that a partial localization of all three AZPs occurs on both the cis-Golgi compartment and the trans-Golgi 
network compartment, which could be caused by the $19^{\circ} \mathrm{C}$ block and may result in AZP mislocalization due to overcrowding of blocked proteins or overlapping of Golgi lamellas that are unable to maintain their natural structure through the balance of fusing and budding vesicular structures.

The $19^{\circ} \mathrm{C}$ block allowed detection of endogenous AZPs through the Golgi stack with confocal microscopy, but it does not reflect normal localization conditions and indicates the need for AZP localization studies with another microscopy technique that does not require a similar temperature block.

\subsubsection{Localization of AZPs to Golgi sub-compartments with and without a $19^{\circ} \mathrm{C}$ block, at and on the Golgi lamella (STED)}

To visualize the localization of AZPs at Golgi substructures, with a different microscopy technique and in more detail, I repeated the localization experiment under both normal $\left(37^{\circ} \mathrm{C}\right)$ and $19^{\circ} \mathrm{C}$ block conditions, with super resolution microscopy. Munc13-1 was co-immunostained with the cis-Golgi marker GM130 and Bassoon and Piccolo were stained with the trans-Golgi marker TGN38.

All three proteins were visualized as punctate signals at their respective substructures (Figure 4). This is a striking observation, as light and confocal microscopy techniques of the localization of these proteins have typically shown AZP signals in the soma that are visually similar to those of the Golgi lamella. The increase in resolution is similar for both channels labelled for either the Golgi marker or the AZP and the localization of both type of proteins are visualized using a primary antibody (10-15nm in size) and a secondary antibody (10$15 \mathrm{~nm}$ in size) complex, that generates a $30 \mathrm{~nm}$ signal around each labeled molecule ${ }^{94}$, allowing for similar-sized signals being observed. These signals can be resolved from neighboring signals as the resolution limit regularly obtained by the STED setup used for this experiment was between 40 and $60 \mathrm{~nm}$ for both channels $^{88}$. As the size of one AZP or Golgi molecule signal is similar, observing AZPs as punctate signals that are visually dissimilar to the Golgi marker staining signifies the presence of a low density of AZP signals that are being specifically distributed over their corresponding Golgi compartments. To take a closer look at the distribution of the AZP to their Golgi sub-compartment, I generated masks in each image representing a control region in the nuclear region of the soma (blue), a mask representing the area occupied by the Golgi lamella (yellow), and a mask for the regions between the Golgi lamella (white). I calculated the AZP signal populations $/ \mu \mathrm{m}^{2}$ area, in these mask regions for every image, in the $19^{\circ} \mathrm{C}$ blocked and $37^{\circ} \mathrm{C}$ condition sets. A higher number of all three AZP were observed in the $19^{\circ} \mathrm{C}$ blocked images at their respective Golgi sub-compartments in all mask regions. In particular, 13.7-times-higher Bassoon signals (from 0.14 to 1.94 puncta $\left./ \mu \mathrm{m}^{2}\right)$, 1.98-times-higher Piccolo signals $\left(0.74\right.$ to 1.47 puncta/ $\left.\mu \mathrm{m}^{2}\right)$ and 2.96-times-higher Munc13-1 signals (from 0.7 to 2.1 puncta/ $\mu \mathrm{m}^{2}$ ) were 
observed in the control regions of normal and $19^{\circ} \mathrm{C}$ blocked images (Figure 4). These results reflect the presence of a large load of AZP signals, arrested on all Golgi sub-compartments during a $19^{\circ}$ block.

Using STED microscopy, AZP localization in normal $37^{\circ} \mathrm{C}$ conditions was clearly discernable without a block in all three mask regions. To further discern the natural distribution patterns of the different AZP to their Golgi sub-compartment, images from the $37^{\circ} \mathrm{C}$ condition set were compared. All three AZP have higher frequencies at and between Golgi lamella regions when compared to their control region, though there appears to be no significant distribution pattern of AZPS favoring localization between the at- lamella and between-lamella masks (Figure 4).

These results show that AZPs Bassoon, Piccolo, and Munc13-1 appear as punctate signals, which do not resemble Golgi marker signals in the soma, and are localized at and around trans-Golgi network lamellae and cis-Golgi lamellae, respectively. The similar distributions of AZPs in close association to Golgi lamellae and next to them, is presumably a population AZP signals consecutively being recruited to the Golgi lamella to be loaded onto transport precursors that begin their transit out of the soma. These distribution patterns and localizations of AZPs were only discernable, without a transport block, using super resolution microscopy. The association of AZP subsets to specific Golgi substructures provides a means by which to characterize distinct transport precursors arising from the Golgi, for example, transport vesicles that bud from the cis-Golgi membrane transport Munc13-1 and trans-Golgi network-derived vesicles carry Bassoon, Piccolo, and ELKS2, of which a fraction of vesicles must be PTVs. These results illustrate the earliest presence of AZPs, at distinct cellular substructures in young neurons, and highlight the Golgi as the first modulatory station in their journey to the synapse.

Figure 4: Distribution and localization of AZPs to their respective Golgi sub-compartments with and without a $19^{\circ} \mathrm{C}$ block using STED microscopy.

Super resolution imaging of DIV6 hippocampal somas that were immunostained for Bassoon and Piccolo with TGN38, and Munc13-1 with GM130, in neurons kept at $37^{\circ} \mathrm{C}(\mathrm{A}, \mathrm{C}, \mathrm{E})$ and $19^{\circ} \mathrm{C}(\mathrm{B}$, $\mathrm{D}, \mathrm{F})$, before paraformaldehyde fixation. Blue outline of image area represents a control region in nucleus of the cell, the yellow outlines represent the area occupied by the lamella of the Golgi markers in the image, and the white outlines represent the image areas between the lamella of the Golgi signals. The number of puncta per $1 \mu \mathrm{m} 2$ area of the AZPs were quantified at all three regions of interest at $37^{\circ} \mathrm{C}$ and $19^{\circ} \mathrm{C}(\mathrm{G}, \mathrm{H}, \mathrm{I})$. Data were statistically tested with a one-way annova and a post hoc Tukey's multiple comparison's test where ${ }^{*} p<0.05,{ }^{* *} p \leq 0.01$, and ${ }^{* * *} p \leq$ $0.001, \mathrm{~N}=20$ cells from two separate sets. 


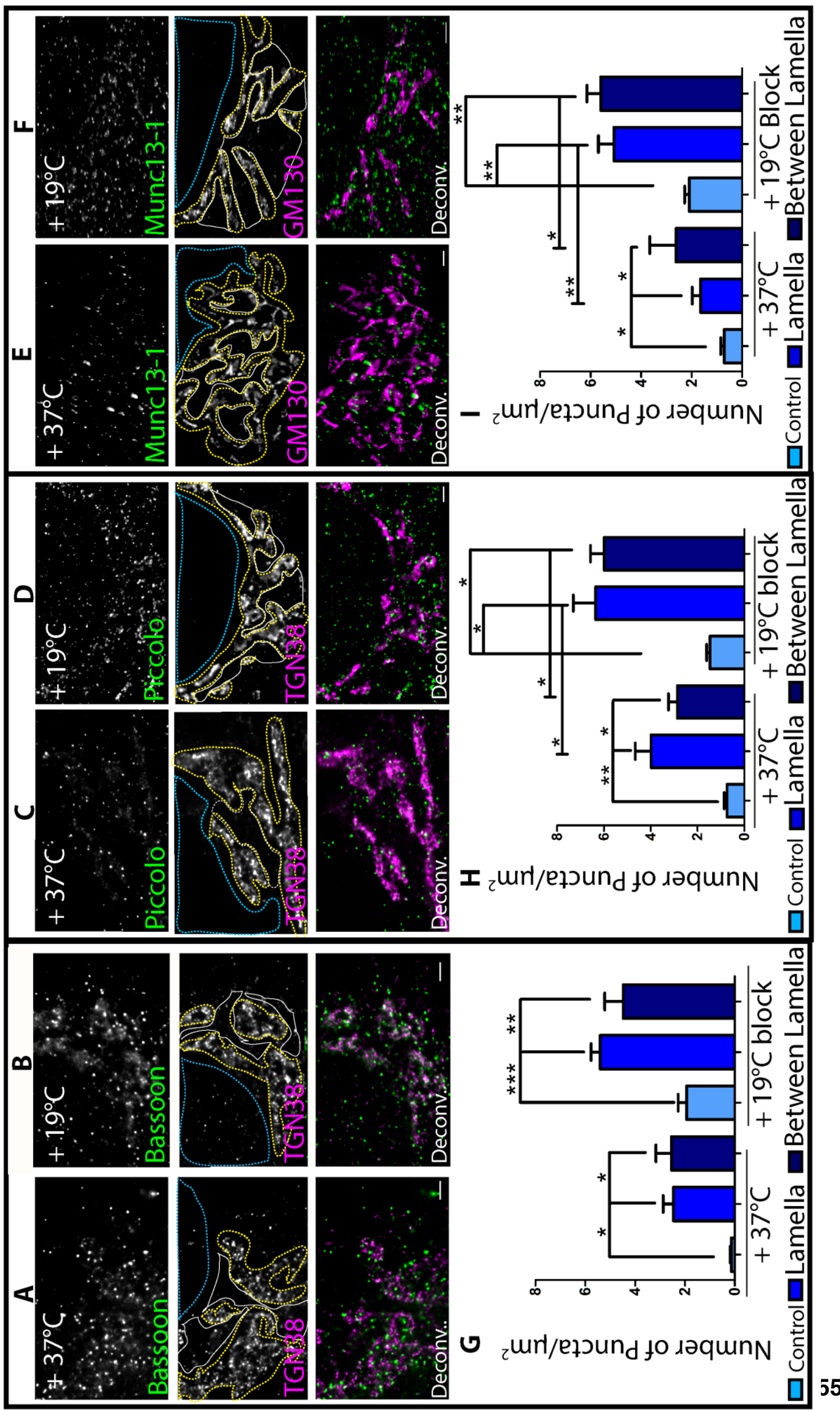




\subsection{Transport and localization of endogenous and recombinant Bassoon constructs}

The soma of a neuron is a modulatory site at which transport vesicles are generated and organized. The vesicles generated at different Golgi compartments can carry a specific subset of AZPs into the axon. The type of the vesicles that AZPs are loaded onto may affect their assembly at the synaptic sites.

Endogenous Bassoon is localized to the trans-Golgi network, with ELKS2 and Piccolo $^{32}$. In addition a large number of studies employing the use of recombinant Bassoon constructs, including the well characterized tagged 95-Bsn construct, localize to TGN marker with light and confocal microscopy ${ }^{30,70}$, while unpublished data from our lab show EM micrographs of the same tagged 95-Bsn construct predominantly loaded onto clear-core vesicles in the soma (Dresbach and Wittenmayer, unpublished). These proteins were isolated in the same biochemical fraction that contained dense-core vesicles and were defined as $\mathrm{PTVs}^{69}$. Electron microcopy and live imaging of endogenous Bassoon has shown evidence of the protein localized on transporting dense-core vesicles as well as between a package of dense and clear-core vesicles in the axon ${ }^{6,95,96}$. Despite this information, a lot still remains unclear about the biogenesis, composition, and mechanism of their transport that attribute to CAZ assembly. For example, no one knows whether clear-core and dense-core vesicles arise from different subcompartments of the Golgi carrying a different AZP subset. Subsequently, it is also unresolved whether AZPs are co-assembled before their transport down the axon or whether they get co-assembled en route.

To fill in the gaps of knowledge that these questions raise, I will characterize the following:

(1) the types of AZP transport carriers that arise from the cis-Golgi and the trans-Golgi network compartments positive for Munc13-1 and Bassoon/Piccolo, respectively;

(2) the distribution of the AZPs in different regions of a developing axon;

(3) the composition of co-trafficking AZPs in axons with super resolution microscopy;

Together, these questions will help characterize the link between AZP localizations and their transport precursors as well as cast light onto possible modulatory steps during transport that might influence $A Z$ assembly. 


\subsubsection{Transport entities generated at the soma}

\section{Signal sizes of AZP transport packets at the Golgi}

To characterize the different transport precursors generated at different Golgi compartments, I quantified the AZP signals that might reflect the size of transport vesicles they are transported on. Endogenous Bassoon, Munc13-1, and Piccolo were labeled with traditional antibodies and imaged with a two-color STED microscope at their respective Golgi compartments. A range of AZP signal sizes from 50nm-150nm were seen for Bassoon, Piccolo, and Munc13-1 at all Golgi compartments. This implies that any AZP, irrespective of the Golgi lamella that it was processed on, can be loaded on a range of different vesicles sizes. As AZP signals around the Golgi lamella could be vesicles in transit, a population of AZP signals, might also be loaded onto permutations of vesicle clusters in the soma, to promote their efficient transport to nascent synaptic sites.

To test the distribution of the potential AZP transport precursors generated, all the AZP signal sizes in an image were measured and grouped in three size categories. These categories group the AZP signals into puncta diameter size ranges of $30-65 \mathrm{~nm}$ (consistent with clear core vesicles sizes such as synaptic vesicles), 66-95nm (consistent with dense core vesicles such as PTVs) and 96-150nm (representing small clusters of vesicles that may contain dense- and clear-core vesicle packages). The populations of signals present in these size ranges were plotted as a percentage of the total number of signals per image. Signal sizes greater than $150 \mathrm{~nm}$ in diameter were rarely seen, for any AZP staining and the few that were observed were excluded on account of being size distribution outliers. Although AZP proteins are known to load on top of transport vesicles, the size of their signals may not accurately represent the size and type of the transport vesicle they are loaded on. As double-colored STED images do not provide a high resolution in the z-axis, it possible that AZP signals may appear erroneously large, due to the presence of out of focus signals below them in the z-plane. Nevertheless, the striking presence of a visible variety of signal ranges may elude towards the presence of a range of AZP transport precursor types.

A detailed look at the population of AZP signals reveals a significantly larger fraction of the total population of signals of Bassoon (51.3\%), Piccolo (46.8\%), and Munc13-1 (48.7\%) in the 96-150nm size range, which is indicative of small vesicle clusters (Figure 3). Interestingly, the distribution of AZP signals, irrespective of the type of AZP and its localization to a specific Golgi compartment, is similar for all AZP size categories. Images of all three AZPs reveal distributions of around $15-16 \%$ of the total AZP signal size population that corresponds to the clear-core transport precursor category, around $32-35 \%$ of the population that corresponds to the dense-core category, and $47-51 \%$ of AZP population that corresponds to small vesicle cluster category. This uniform 
distribution of AZP signals point towards the presence of a pool of different vesicle types, available around Golgi structures, for AZPs to be indiscriminately loaded onto. Meanwhile, since the largest fractions of AZP signals are in the 96$150 \mathrm{~nm}$ range, a trend toward the formation of small AZP transport vesicle clusters in the soma is expected. This arrangement of transport precursors could be a sorting mechanism that prerequisites early transport in young neurons (Figure 5).

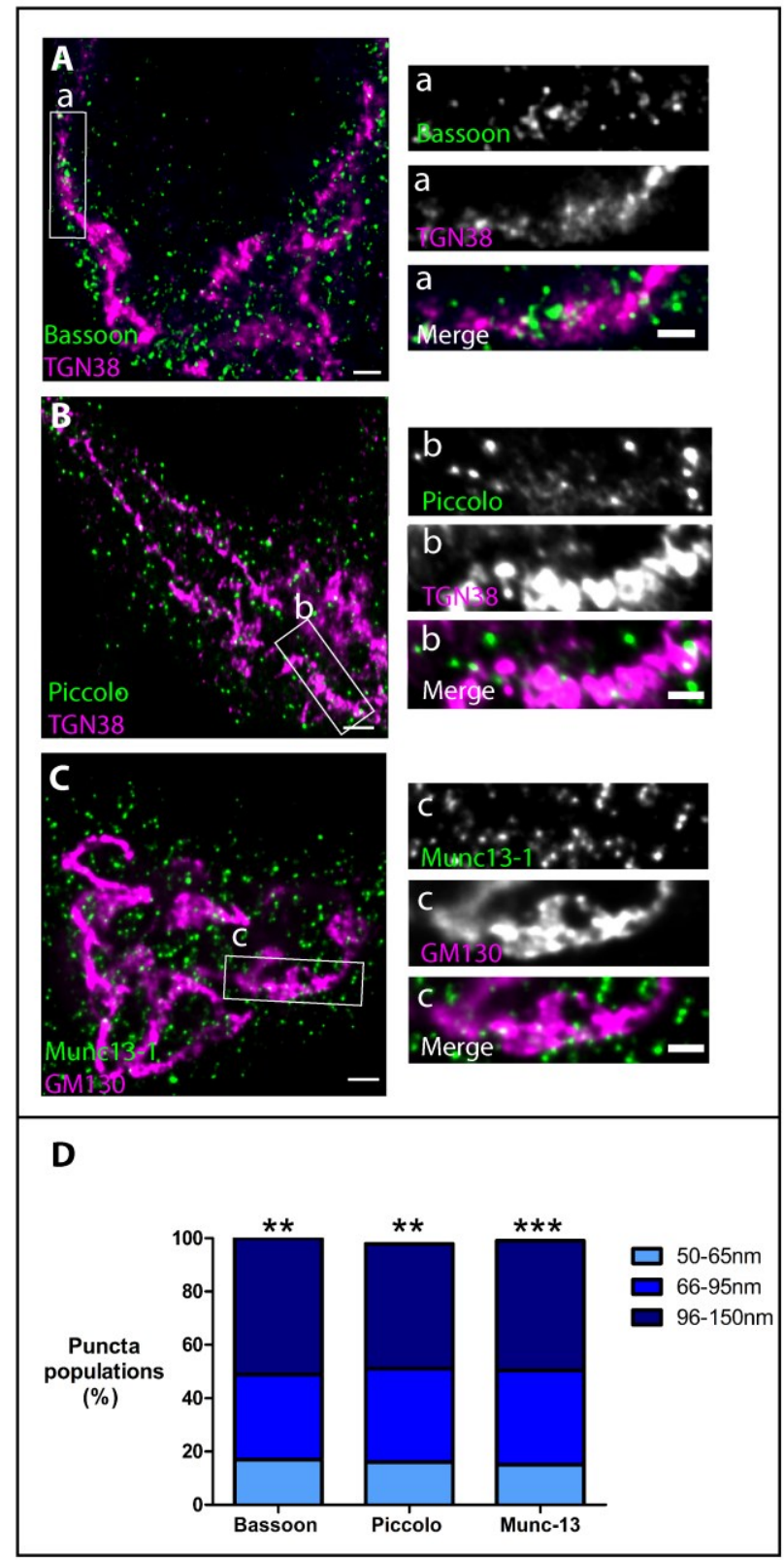

Figure 5: Size populations of endogenous Bassoon, Piccolo, and Munc13-1 signals at their Golgi sub-compartments. A-C, Two-color STED images of DIV6 hippocampal neuron cell somas immunostained with antibodies against Bassoon (A, a), Munc13-1 (B, b), Piccolo (C, C), at TGN38 or GM130. ROls $\mathbf{a}, \mathbf{b}$, and $\mathbf{c}$ represent a range of punctate signals that were measured for their size and grouped in three size categories that represent known clear-core vesicles (50$65 \mathrm{~nm})$, dense-core vesicles $(66-95 \mathrm{~nm})$ and small vesicle cluster sizes $(96-150 \mathrm{~nm})$. Data are represented as a percentage of the total population of signals per image $\mathrm{N}=9$ cells from three separate sets. Scale bars $(\mathbf{A}-\mathbf{C}), 1 \mu \mathrm{m}$. 


\section{Characterizing type of transport packets of tagged Munc13-1 and Bassoon in the soma}

Apart from their characteristic 80nm diameter size, dense-core vesicles are known to transport larger molecular weight neurotransmitters and a range of different neuropeptides such as BDNF and Chromogranin A/B (CGA / CGB) ${ }^{71,97}$, by which they can be characterized. Additionally, large dense-core vesicles are known to possess the synaptotagmin class of transmembrane proteins in their vesicle lipid bilayer. Synaptotagmin4 (Syt4) is one such vesicular transmembrane protein that has been shown to be present at the Golgi compartment, on large dense-core vesicles of differentiating PC12 cells and neurons ${ }^{97,98}$, making it a good candidate to label dense-core transport precursors in the soma.

Figure 6: Early localization of recombinant Bassoon and Munc13-1 to dense-core vesicle marker: Synaptotagmin4 (Syt4). DIV7 hippocampal neurons were transfected for Bassoon (95-3938 construct) (panels A), and Munc13-1 (panels B) were immunostained for syt4 and visualized in neuronal somas using confocal microscopy. Zoom images of $\mathbf{A}$ and $\mathbf{B}$ over view images (I) and inset of I represented in single channels and merge as images II. Arrows represent colocalizing signals. Scale bars over view images $10 \mu \mathrm{m}$ and $5 \mu \mathrm{m}$ (I and II zooms).
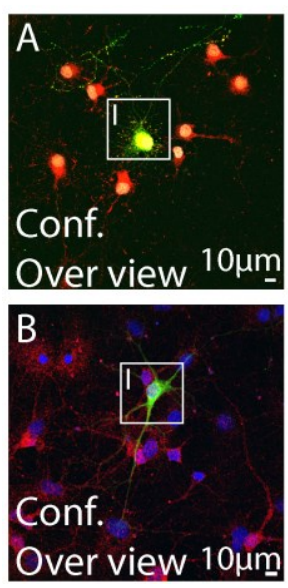
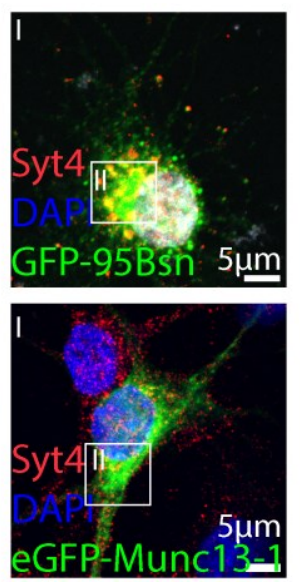
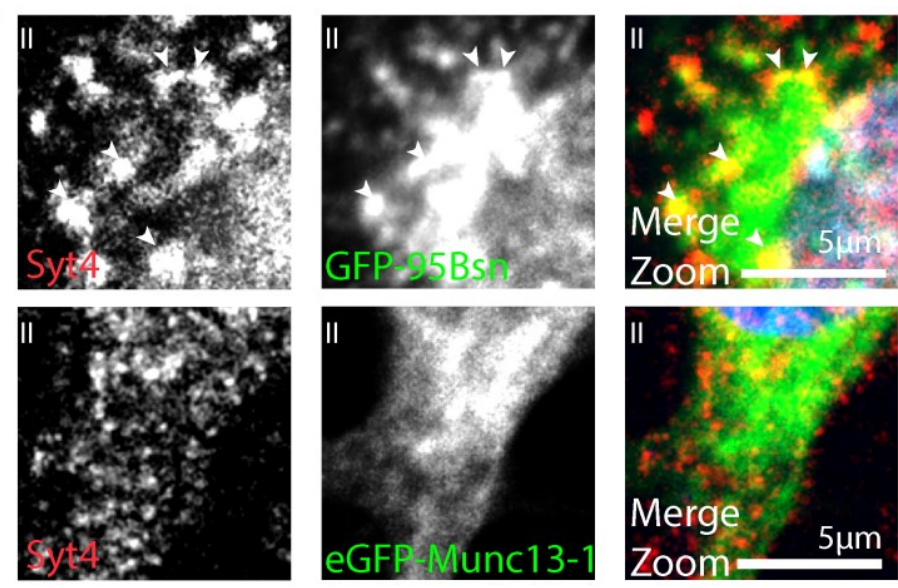

In order to ascertain the presence of dense-core vesicles arising from the Munc13-1 (cis-Golgi) and Bassoon (trans-Golgi network) positive compartments, I co-immunostained for Syt4 in order to label AZP transport precursors that possess a dense-core and are close to the Golgi. Colocalization of tagged GFP95-Bassoon and eGFP-Munc13-1 was visualized in the somas of young (DIV7) neurons by confocal microscopy. Little to no visual colocalization of Munc13-1 was seen with the Syt4 marker, suggesting that Munc13-1 is not loaded onto Syt4-positive dense-core vesicles. In comparison, a subpopulation of the clusters of Bassoon signals in the soma was visibly colocalized to Syt4-positive densecore vesicles (Figure 6).

These results together and in the light of available information in field, impress upon the presence of a large range of available transport carriers that may transport an AZP to its final destination. These transport vesicles not only vary in size of AZP signals they carry but also in the composition of their cargo, although 
the distribution and availability of this diverse vesicle population is evidently uniform for all AZPs, irrespective of the Golgi lamella from which they were loaded. Further characterization of the subtypes of AZP-specific transporter precursors is required. However, this is beyond the scope of this investigation at this point, due to the lack of functional vesicle subtype markers and the complex diversity of existing vesicle subtypes that are not fully characterized in hippocampal neurons.

\subsubsection{Distribution of endogenous Bassoon to other} AZPs in axons of developing neurons undergoing

\section{trafficking}

The exact process of how a sufficient subset of all AZPs is recruited to an assembly site of an $A Z$ is unknown, though it is believed that a preassembly of AZPs may occur before and/or during their transport down the axon. Results from section 4.4.1 show large populations of AZP signals in size ranges, reflecting those of grouped vesicles. This suggests that different AZPs may come in close proximity for preassembly at the soma, and might also do so during transport. To visualize the colocalizations of AZPs to each other during transport, I began by characterizing their distribution in the axons of developing neurons.

\section{Distribution of endogenous AZPs in different regions of the developing axon}

Epifluorescence microscopy was used to determine the endogenous distribution of Bassoon, Piccolo and Munc13-1 in three, identical, regions of interests selected in a proximal region of the axon, a central region of the axon, and a distal axonal region of young DIV6 hippocampal neurons. To visualize the varying distribution of AZPs to one another, I co-immunostained Bassoon with Munc13-1 and Bassoon with Piccolo and determined their colocalization in the regions of interests.

High colocalization frequencies of Bassoon are seen with Munc13-1 (55.2\% at proximal axon, $57.8 \%$ at central axon, and $66.6 \%$ at distal axon) and Piccolo (64.7\% at proximal axon, $49 \%$ at central axon, and $53.6 \%$ at distal axon), in all regions of interest. Although interestingly neither region of interest has a significantly higher colocalization of Bassoon with Munc13-1 or Piccolo (Figure 7). 

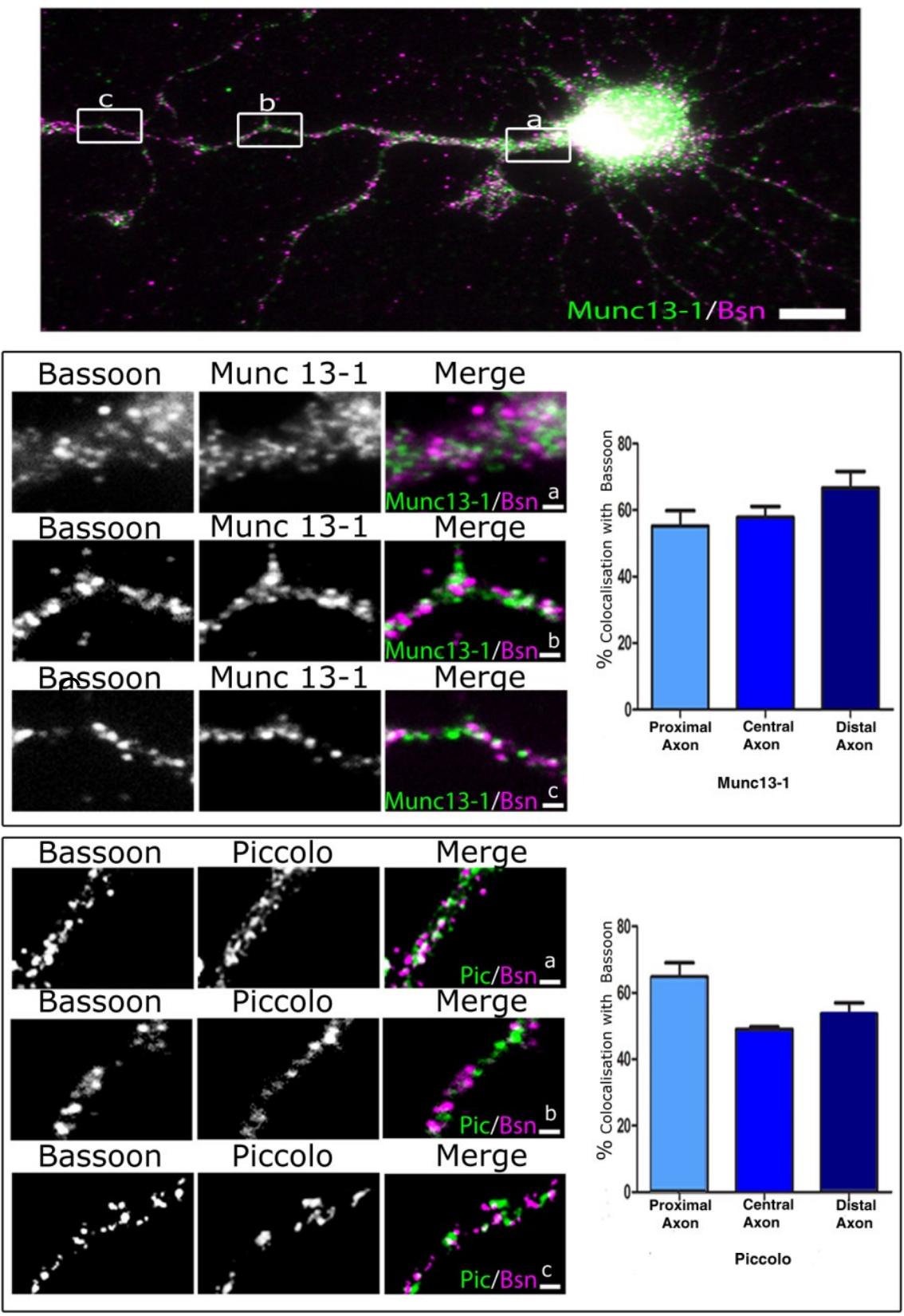

Figure 7: Distribution of Bassoon, Piccolo and Munc13-1 in developing neurons with epifluorescence microscopy. A, over view image of DIV6 hippocampal neurons immunostained with Bassoon and Munc13-1 (B) and Bassoon and Piccolo (C) in three axonal regions: the proximal axon $\mathbf{a}$, the central axonal region $\mathbf{b}$ and the distal axon $\mathbf{c}$ and quantified for the amount of colocalized Bassoon. Each axonal ROI is off size. Data represented as mean \pm SD, $N=20$ cells from two separate experiment. Scale bars $10 \mu \mathrm{m}(\mathbf{A}) 1 \mu \mathrm{m}$ (B and $\mathbf{C})$.

This signifies that AZPs are uniformly distributed throughout the developing axon and AZPs don't appear to localize at specialized sites in the axon, to preassemble during transport. 


\section{Localization of endogenous Bassoon to other AZPs in the developing axon}

To visualize in detail the spatial localization of AZPs to one another, in the axons of young neurons, endogenous Bassoon-Piccolo and Bassoon-Munc13-1 coimmunostainings were imaged with confocal microscopy and two-color STED in a central axonal region. A significant drop in the percentage of colocalized Bassoon was observed when comparing confocal images (67.8\% with Piccolo and $50.2 \%$ with Munc13-1), to the STED images (36.4\% with Piccolo and $30.9 \%$ with Munc13-1) (Figure 8). This suggests that a large fraction (approximately $70 \%$ ) of the Bassoon population, being transported down the axon of developing neurons, travels on transport entities that do not contain Piccolo or Munc13-1.

Figure 8: Distribution of Bassoon, Piccolo and Munc13-1 during high neuronal trafficking using STED microscopy. DIV6 hippocampal neurons immunostained with Bassoon and Piccolo (A and B), or Bassoon and Munc13-1 (C and D), in central axonal regions, imaged using confocal and STED microscopy on the same area. Quantification of the amount of colocalized Bassoon with Piccolo (E) and Munc13-1 (F) Data represented as mean $\pm S D, N=20$ cells from two separate experiment, ${ }^{*} p<0.05$. Scale bars $1 \mu \mathrm{m}(\mathbf{A}-\mathbf{D})$.

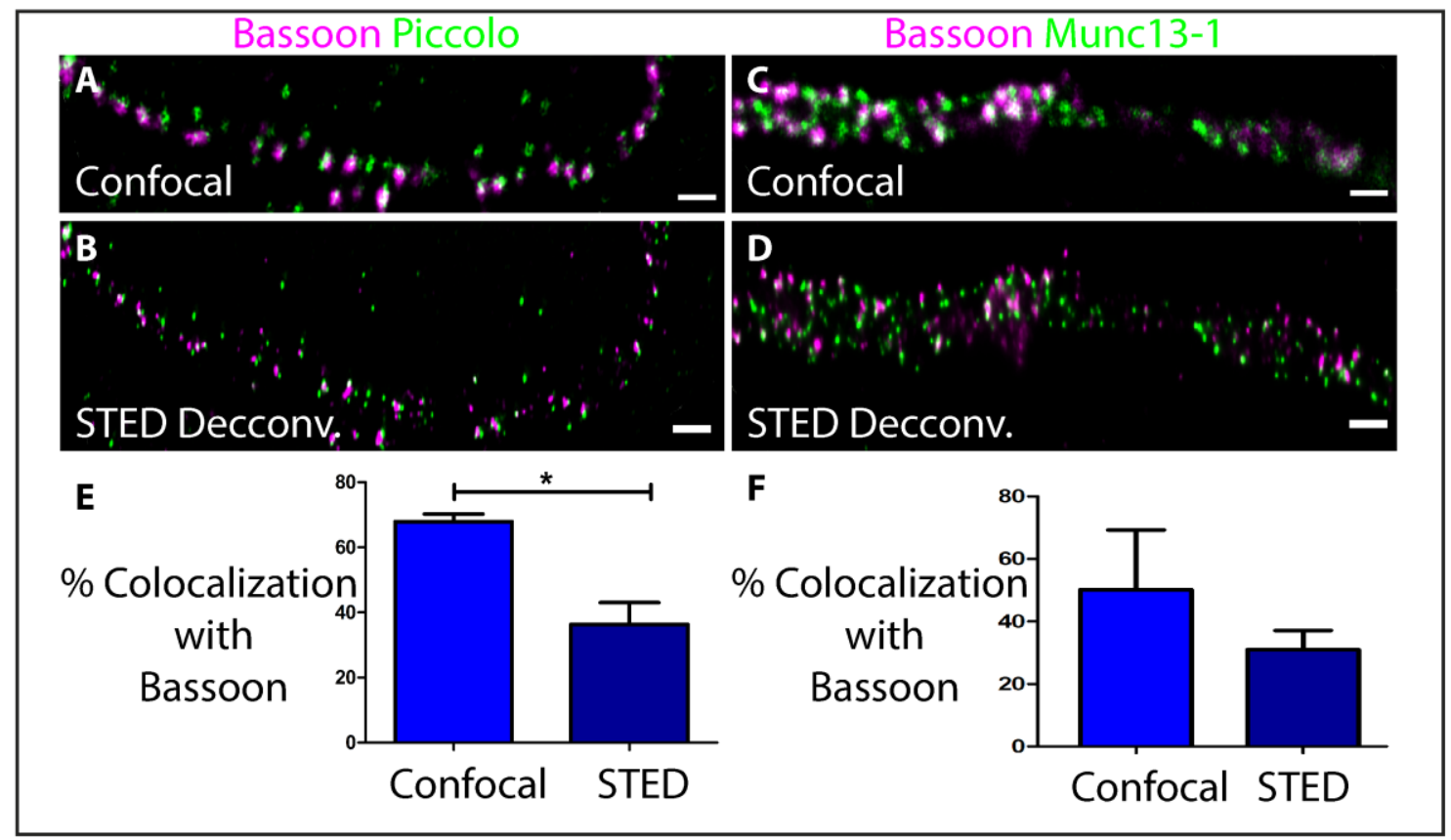


This result highlights the fact that during transport the majority of AZP protein populations in the axon preferentially traffic on their own pre-assigned transport carriers and rarely begin co-trafficking with other AZP signals in the axon.

These highlight the presence of a uniform distribution of AZPs in axons, which in turn indicates that AZP carrying transport vesicles do not accumulate at a specific region in the axon to organize an exchange of AZPs during transport. Super resolution imaging of $A Z P s$ in the soma reveals the predominant presence of AZP signals sizes on corresponding sizes of small groups of transport precursors, which may transport only one or a subset of AZPs. This suggests that combinations of AZP compositions on transport precursors may already be arranged in the soma prior to their transport out of the soma. This result is compounded by the fact that in the axon, super resolution microscopy reveals only $30 \%$ of the trafficking Bassoon population in close proximity to other AZPs. These $30 \%$ of colocalizing AZP signals could represent co-trafficking vesicles that are either prearranged in the soma or have become associated with each other during transport. These co-trafficking events might therefore bring different AZP compositions in close proximity, in an anticipatory first step towards AZP preassembly, at sites on the axon that might become immature synaptic sites.

Together the results from this section suggest that subsets of AZPs on transport precursors may be prearranged in the soma, which may additionally come in close proximity to a different subset of AZPs during co-trafficking events, where it seems unlikely they would begin to preassemble into immature AZ. Nonetheless the close proximity of co-trafficking vesicles carrying different AZP subsets, might aid in the efficient recruitment of a sufficient group of AZPs to nascent synapses 


\subsection{Characterization of new full-length Bassoon constructs}

It is becoming increasingly evident that to have a better understanding of the assembly of AZPs, the localization of AZPs at their modulatory check points need to be studied in higher detail. Underlying specific details such as the orientation of an AZP and its interaction to membranes of the Golgi lamella, transport vesicles or the presynaptic membrane, may reveal temporal modifications that allow for AZ assembly and improve knowledge about their localization.

Results in section 3.1 highlight the need to visualize AZP molecules at higher resolutions, although determining the localization of endogenous AZPs, requires traditional mono- and polyclonal antibody complexes for visualization, which in turn limits the understanding of AZP localization to $30 \mathrm{~nm}$ from its true location ${ }^{94}$. Furthermore, the use of traditional poly- and monoclonal antibodies generate large antibody complexes that are believed to create steric problems that prevent unbound antibody molecules from reaching all the epitopes and thereby perpetuates artificial staining ${ }^{80}$. Expressing tagged recombinant protein allows the use camelid antibodies, which are small enough to reduce the steric problems.

The emergence of fab fragments and nanobodies have helped reduce the problem of large antibody signals, although at this stage, specific AZP products of such nature are not available. Alternatively, the use of GFP and RFP nanobodies has been proven to improve localization of tagged proteins ${ }^{82,83,85}$. Thankfully a wide variety of tagged AZP constructs have been generously used and well characterized in the field ${ }^{30,70,99,100-102,47}$. This provides a great platform for me to employ, already available and freshly generated tagged AZP constructs and their deletion mutant variants to visualize in further detail the localization, orientation and organization of AZPs, en route to forming matured synapses.

The largest range of full-length and well-documented AZP deletion constructs available today are that of the Bassoon molecule. First-generation recombinant Bassoon constructs have been widely used for trafficking, AZ assembly and maintenance studies in the field, and provide a strong background for the application of these constructs ${ }^{30,70,99}$. Additionally, new second-generation Bassoon constructs, which will be characterized in this section, have the advantage of being the first full-length constructs optimally tagged to visualize the orientation of the protein at different subcellular localizations. Ultimately, the application of Bassoon recombinant constructs have the advantage of being easily administered via transfections in cultured neurons and having their tags readily modified for a range of interaction assays and cutting-edge imaging techniques that provide the opportunity to visualize these AZPs in nanoscopic detail and follow their transport via live imaging. 
Bassoon is also one of the two largest proteins in the $A Z$ complex $^{22,52}$; making it an ideal candidate to study AZP localization and orientation, and one of the first $\mathrm{AZPs}^{99}$ to be recruited to a young synapse, placing the molecule center stage during CAZ organization. All these factors contribute and compliment the choice of employing first- and second-generation Bassoon constructs to the study of localization, orientation, and organization of AZPS on their journey from the TGN to the synapse.

In this section, I will introduce new second-generation single- and double-tagged full-length Bassoon constructs that were recently generated in the lab as the tools to visualize the nanoscopic localization and orientation of Bassoon. These new constructs carry an intramolecular mEGFP/mRFP at the 97 amino acid position (adjacent to its $\mathrm{N}$ terminus), leaving the $\mathrm{N}$-myristolyation (MGNEASLEG) consensus site of Bassoon intact. Additionally, the constructs carry a C-terminal $\mathrm{mEGFP/mRFP}$ tag that is optimized, by the removal of Bassoon's stop codon, to increase the stability of constructs (Figure 9).

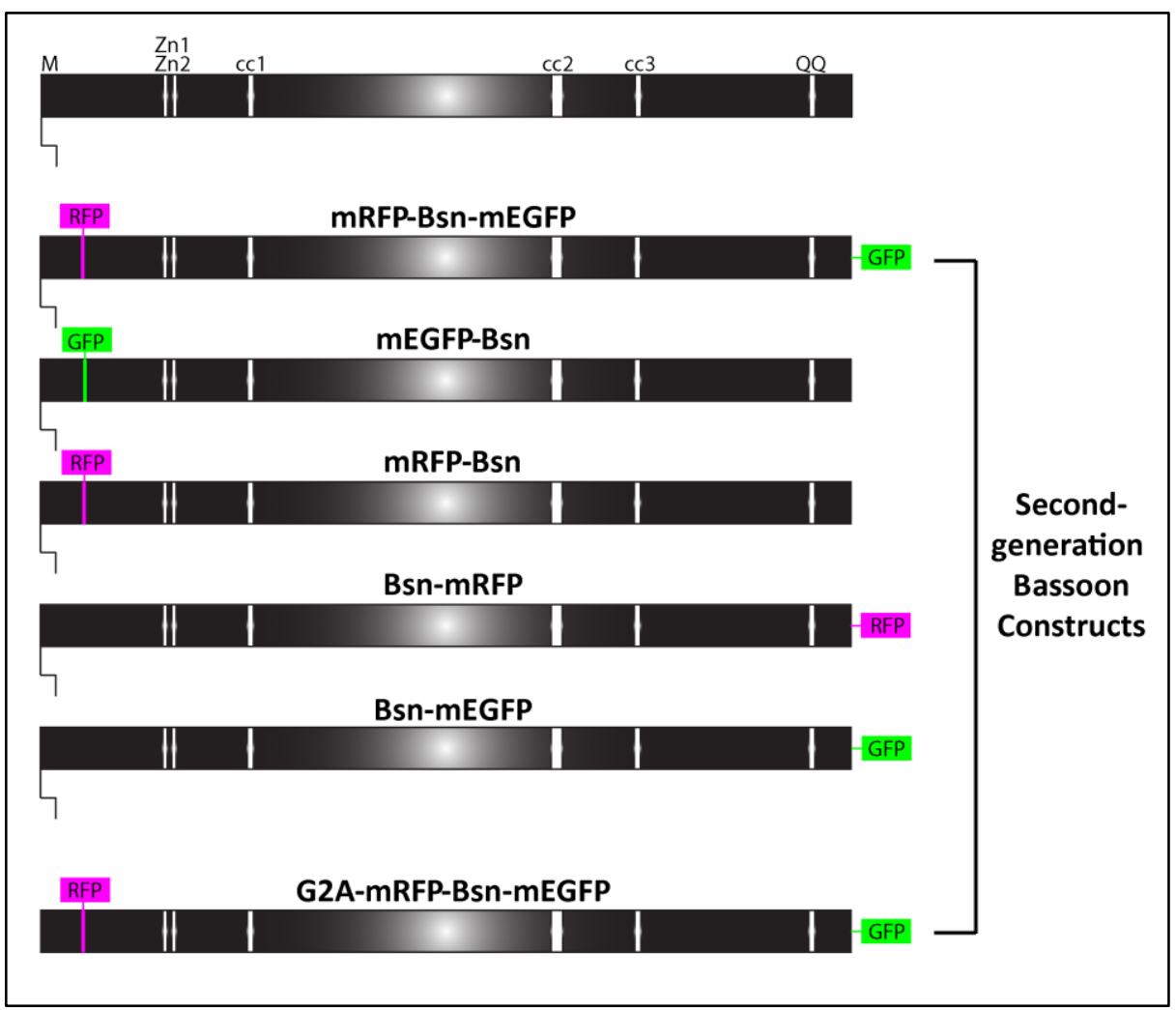

Figure 9: A schematic of the second-generation Bassoon constructs used in this study. A map of Bassoon (1-3938 amino acids) protein consisting of a myristoyl motif (M); zinc finger domains (Zn1, Zn2); and coiled-coil domains (cc1_cc3) can be used to compare the generated constructs. Five full-length double- and single-tagged constructs and a double-tagged myristoyl motif missing G2A mutant were generated and tagged with either a mEGFP or mRFP at the N-terminal, $97^{\text {th }}$ amino acid position and/or at the 3937th amino acid position at the C-terminus. 


\subsubsection{Characterization of full-length Bassoon constructs at the Golgi}

Endogenous Bassoon is localized to the trans-Golgi network sub-compartment upon observation with epifluorescence and super resolution microscopy (Figures 3 and 4). To determine whether the second-generation constructs are associated with the correct Golgi sub-compartment and hence are available to be recruited on to correct transport organelles, all constructs were transfected and fixed in young developing neurons (i.e. between DIV5-8; during high trafficking) and costained with the TGN38 marker.

The double-tagged construct was treated with cold methanol for five minutes prior to normal paraformaldehyde fixation to quench autofluorescence of the mRFP and mEGFP tags and was subsequently stained with TGN38 and GFP antibody to visualize the construct. Methanol treatment is known not only to quench autofluorescence but also to wash out soluble proteins from the neuron, though Bassoon signals have been previously shown to be preserved and well characterized in methanol fixed neurons ${ }^{22,70}$.

All five full-length constructs have high-intensity juxtanuclear signals, at the TGN38 marker signal, and are easily visualized with epifluorescence microscopy, without the requirement of a $19^{\circ}$ block (Figure 4). These signals are visibly higher than that of endogenous Bassoon signals as is expected for transfected neurons, which produce many more molecules of Bassoon. Figure 5 reveals endogenous Bassoon signal sizes range up to $150 \mathrm{~nm}$. Bearing this information in mind and allowing for a higher expression level of the fusion protein, and a lower resolution limit of the epifluorescence scope, I meticulously excluded neurons that may have signal clusters in the soma larger than $1 \mu \mathrm{m}$. Neurons that have malformed somas, transfection signals within the nucleus and/or have a high recombinant protein expression in all neuronal processes were all qualified as characteristics representative of an over-expressed neuron. Consequently, from this point on, I picked only medium to low expressing neurons for my experiments (an example of this can be seen in Appendix C), as the intention of this study was to record close to innate protein localization in neurons.

High-intensity signals of medium transfected neurons were observed not only localized close to the TGN but also observed as high intensity punctate signals in the axons and were often seen localized in growth cones, as is representative of young neurons developing towards making synaptic contacts. There appeared to be no noticeable difference in the juxtanuclear localization of the double-tagged construct from the single-tagged ones, the $\mathrm{N}$-terminally tagged constructs from the C-terminally tagged constructs and a difference as a result of the application of either mEGFP or mRFP tags between the single-tagged constructs (Figure 10). 

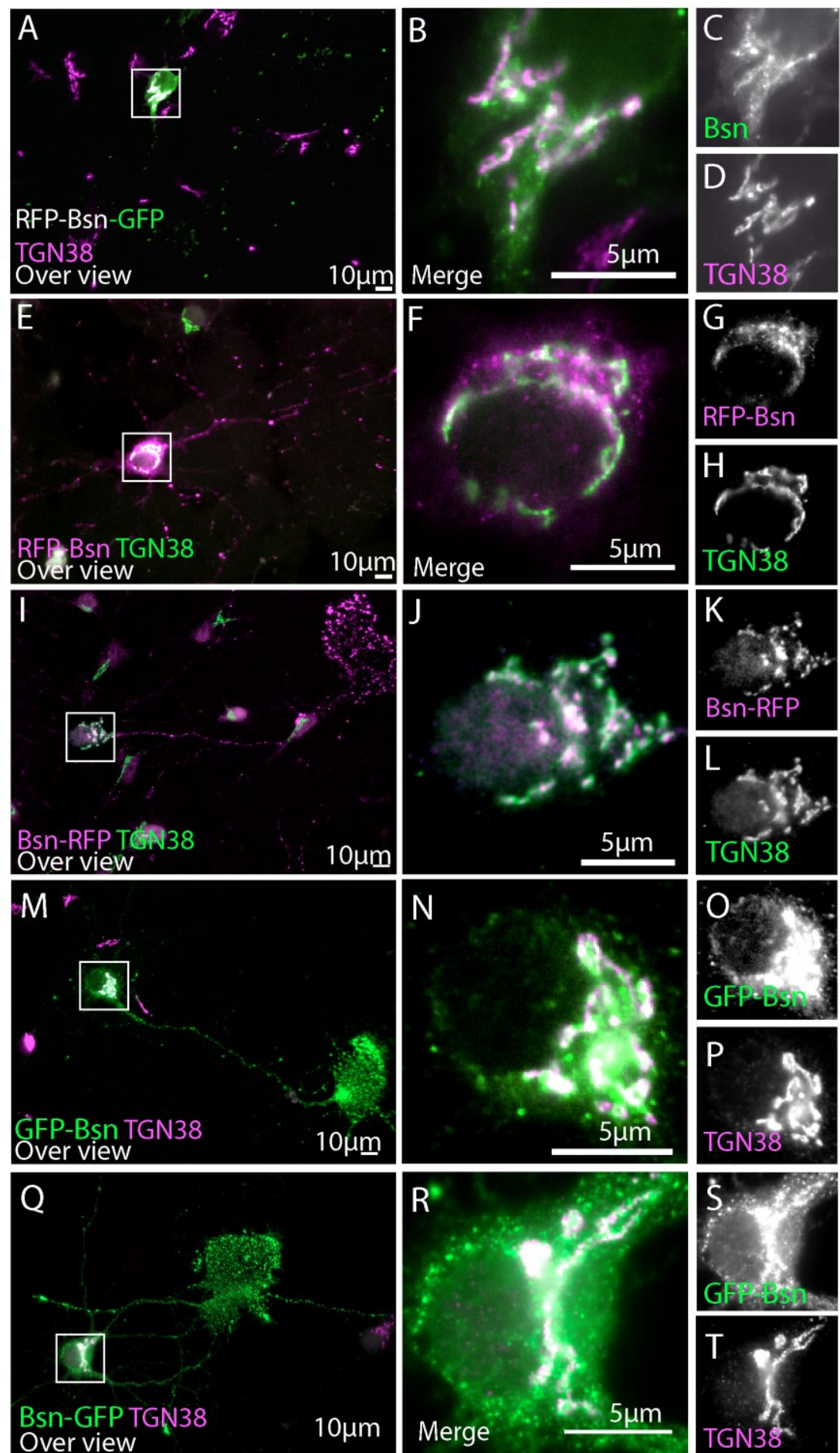

Figure 10: Recombinant Bassoon localizes closely with the TGN38 in young neurons.

A-T, Full-length double-and single-tagged Bassoon constructs were expressed in DIV7 hippocampal cultures, with a DIV6 lipofectamine transfection followed by 24 hour incubation prior to paraformaldehyde fixation and stained with the TGN38 marker. Panels $\mathbf{A}, \mathbf{E}, \mathbf{I}, \mathbf{M}$, and $\mathbf{Q}$ represent 40X over views of the transfections and $\mathbf{B}-\mathbf{D}, \mathbf{F}-\mathbf{H}, \mathbf{J}-\mathbf{L}, \mathbf{N}-\mathbf{P}$, and $\mathbf{R}-\mathbf{T}$ the zooms of their white square insets, respectively. Neurons in panels A-D were briefly fixed in cold methanol prior to normal fixation, to quench RFP and GFP autofluorescence and stained with TGN38 and a GFP antibody. Scale bars $10 \mu \mathrm{m}(\mathbf{A}, \mathbf{E}, \mathbf{I}, \mathbf{M}$, and $\mathbf{Q})$ and $5 \mu \mathrm{m}(\mathbf{B}, \mathbf{F}, \mathbf{J}, \mathbf{N}$, and R). 
These results conclusively show that an accurate localization of the secondgeneration full-length Bassoon constructs was seen in young neurons at the TGN, which situates the protein in the correct sub-compartment to be loaded on to appropriate transport organelles. Furthermore, the localization of the recombinant Bassoon signals into axons and growth cones was indicative of unimpaired early trafficking mechanisms in the neurons and a good sign for the targeting and incorporation of these proteins into synapses.

\subsubsection{Characterization of full-length Bassoon constructs at synaptic sites}

To test whether the new full-length Bassoon constructs target to synapses and get incorporated into the CAZ, calcium phosphate transfection was used to express all five full-length constructs in neurons at DIV3. The subcellular targeting of these fusion proteins were examined post DIV13, at a stage where the neurons had matured and known to possess many stable synaptic contacts ${ }^{90}$. All five constructs were checked for colocalization with a synaptic marker, synaptophysin and Piccolo (a core component of the CAZ). Transfected neurons were co-immunostained post a $0.1 \%$ Triton $\mathrm{X}-100$ extraction.

The AZPs like cytoskeletal proteins have been previously shown to be resistant to the Triton X -100 extraction, as the AZPs become insoluble when incorporated into the CAZ scaffold ${ }^{70}$.

The double-tagged Bassoon constructs, RFP-Bsn-GFP and G2A-RFP-Bsn-GFP, were additionally treated with methanol to quench the autofluorescence of both the tags, between the $0.1 \%$ Triton $X-100$ extraction and normal paraformaldehyde fixation steps. This additional step is necessary as the collective fluorescence of both the tags is detected through F46-002-F46-006 (green, orange, and far-red wavelength) filters available on our epifluorescence scope, leaving no channel free to stain for Piccolo. Fortunately an antibody in the $350 \mathrm{~nm}$ wavelength was available to label against synaptophysin and visualized GFP puncta of RFP-Bsn-GFP were colocalized with synaptophysin at $70.25 \% \pm$ $18.23 \% \mathrm{SD}$, indicating that the double-tagged Bassoon protein correctly targets to synaptic sites. The fusion protein also showed resistance to the Triton X-100 treatment and had a $85.43 \% \pm 18.42 \%$ SD colocalization with the endogenous population of Piccolo puncta present (Figure 11). 

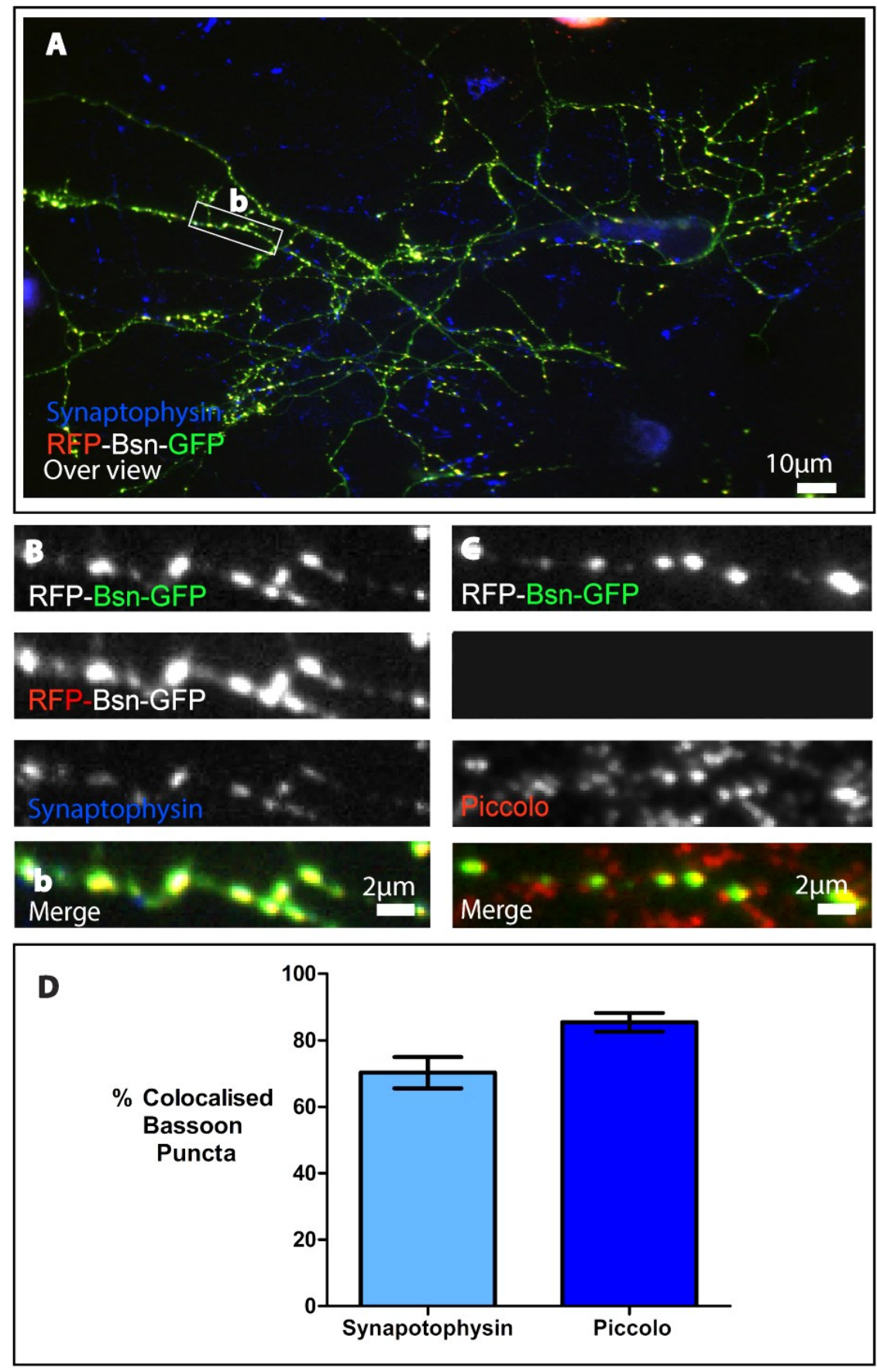

Figure 11: Double-tagged recombinant Bassoon colocalizes with synaptic markers in adult neurons.

A-B, Immunostained DIV14 hippocampal neurons transfected with full-length dual tagged Bassoon construct, post a DIV3 lipofectamine transfection, and stained with the synaptophsyin marker (blue). Zoom of ROI $\mathbf{b}$ is represented in panels $\mathbf{B}$ and panels $\mathbf{C}$ represent a similar ROI for double-tagged Bassoon immuno-labeled for GFP antibody (green) and Piccolo (red) after a 90 second treatment with to $0.1 \%$ Triton $\mathrm{X}-100$ and five minute methanol wash. Graph $\mathbf{D}$. quantification of $(B-C)$; data are represented as mean $\pm S D, N=5$ cells from two separate experiments for each quantification. Scale bars $10 \mu \mathrm{m}(\mathbf{A}) \& 2 \mu \mathrm{m}(\mathbf{B}-\mathbf{C})$. 
Figure 12: Single-tagged recombinant Bassoon also colocalizes with synaptic markers in adult neurons. A-L, Immunostained DIV14 hippocampal neurons transfected with full-length singletagged Bassoon constructs, post a DIV3 lipofectamine transfection, and co-stained with the synaptophysin (A, B D, E, G, H, J, L) or Piccolo marker. M: Quantification of (A-L), data are represented as mean $\pm S D, N=5$ cells from two separate experiments for each quantification.
Scale
bars
$10 \mu \mathrm{m}$
(A)
\&
$2 \mu \mathrm{m}$
(B-C).

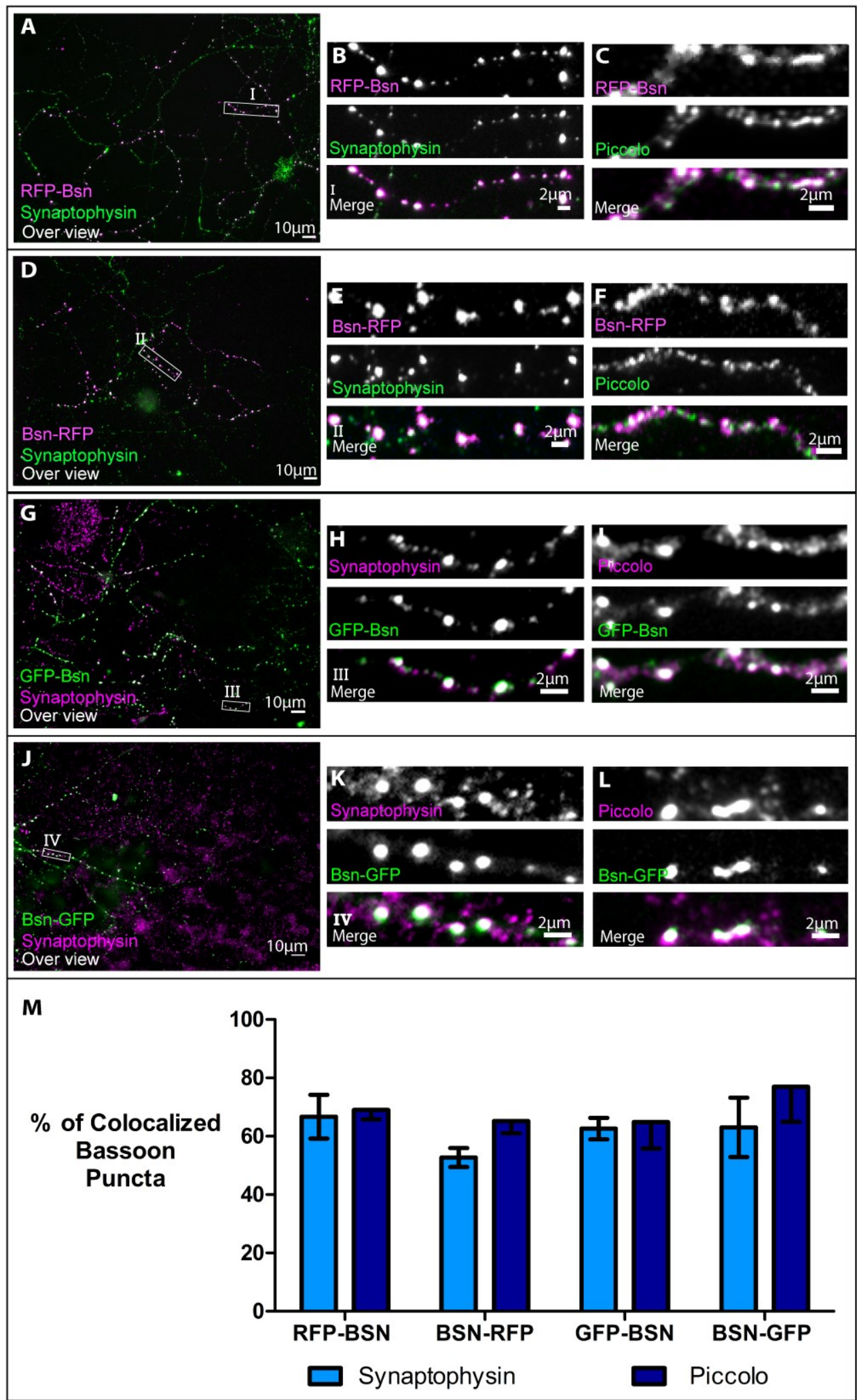


A similar trend was observed for the single-tagged Bassoon constructs where the $\mathrm{N}$-terminally tagged mRFP-Bsn and mEGFP-Bsn constructs had $66.7 \% \pm 12.9 \%$ SD and $62.64 \% \pm 6.37 \%$ SD colocalization with synaptophysin and the Cterminally tagged Bsn-mRFP and Bsn-mEGFP constructs had $52.72 \% \pm 5.57 \%$ SD and $63.02 \% \pm 20.33 \%$ SD colocalization with synaptophysin, respectively (Figure 12). This data suggest that on an average, per image, $63 \%$ of the total tagged Bassoon puncta, were being correctly trafficked and localized to synaptic sites positive for the synaptophysin marker (Figure 12).

Additionally, these recombinant constructs also colocalized to a population of PSD95 or SHANK2 puncta (postsynaptic glutamatergic markers) and strengthen the observation seen with synaptophysin by verifying the correct subcellular targeting of these constructs to active presynaptic sites (Appendix B).

Additionally, the single-tagged constructs mRFP-Bsn, mEGFP-Bsn, Bsn-mRFP and Bsn-mEGFP were also resistant to Triton X-100 and had $69.07 \% \pm 5.62 \%$ SD, $64.85 \% \pm 15.74 \%$ SD, $65.23 \% \pm 7.28 \%$ SD and $76.97 \% \pm 24.01 \%$ SD recombinant Bassoon puncta colocalizing with endogenous CAZ incorporated Piccolo, respectively (Figure 12). On an average, per image, of $72.3 \%$ of tagged Bassoon localized to Piccolo labeled AZs and suggesting that the second generation constructs behaved indistinguishably from the endogenous Piccolo upon Triton X-100 extraction and the first-generation Bsn1-3938 construct (that has a $72 \%$ of GFP puncta colocalization with Piccolo $^{70}$.

These results show that the second-generation Bassoon constructs fully define the properties of the full-length Bassoon protein, as these fusion proteins like the endogenous protein had no nuclear signal and high concentration of juxtanuclear signals in young neurons. These neurons matured with correct targeting and incorporation of Bassoon into the AZ's cytomatrix at presynaptic sites.

\subsubsection{Characterization of full-length Bassoon myristoyl mutant at the Golgi and at synaptic sites}

Myristoylation is a common co-translational lipid modification brought about by the addition of a myristrate consensus via the $\mathrm{N}$-myristoyltransferase (myristoylCoA protein) to the $\mathrm{N}$ terminus of a desired protein. This type of $\mathrm{N}$-terminal acetylation can mediate the interaction between two proteins, facilitate association and binding to membranes ${ }^{103}$. The myristoyl consensus site of Bassoon lies at its second amino acid position as a glycine, which we have point mutated into an alanine. This point mutant destroys the hydrophobic myristoylation modification that may associate Bassoon to various lipid bilayers such as Golgi membranes, transport or synaptic vesicle membranes and cytoskeletal molecules, aiding the targeting and proper orientation of the molecule on its way to the $A Z$. 
In order to test whether the loss of the myristoyl consensus alters the targeting or localization of the Bassoon molecule, DIV6 lipofectamine transfection for Golgi association and DIV3 calcium phosphate transfections, matured in DIV14 neurons to gauge synaptic targeting and $C A Z$ incorporation were performed using the G2A-RFP-Bsn-GFP mutant construct.
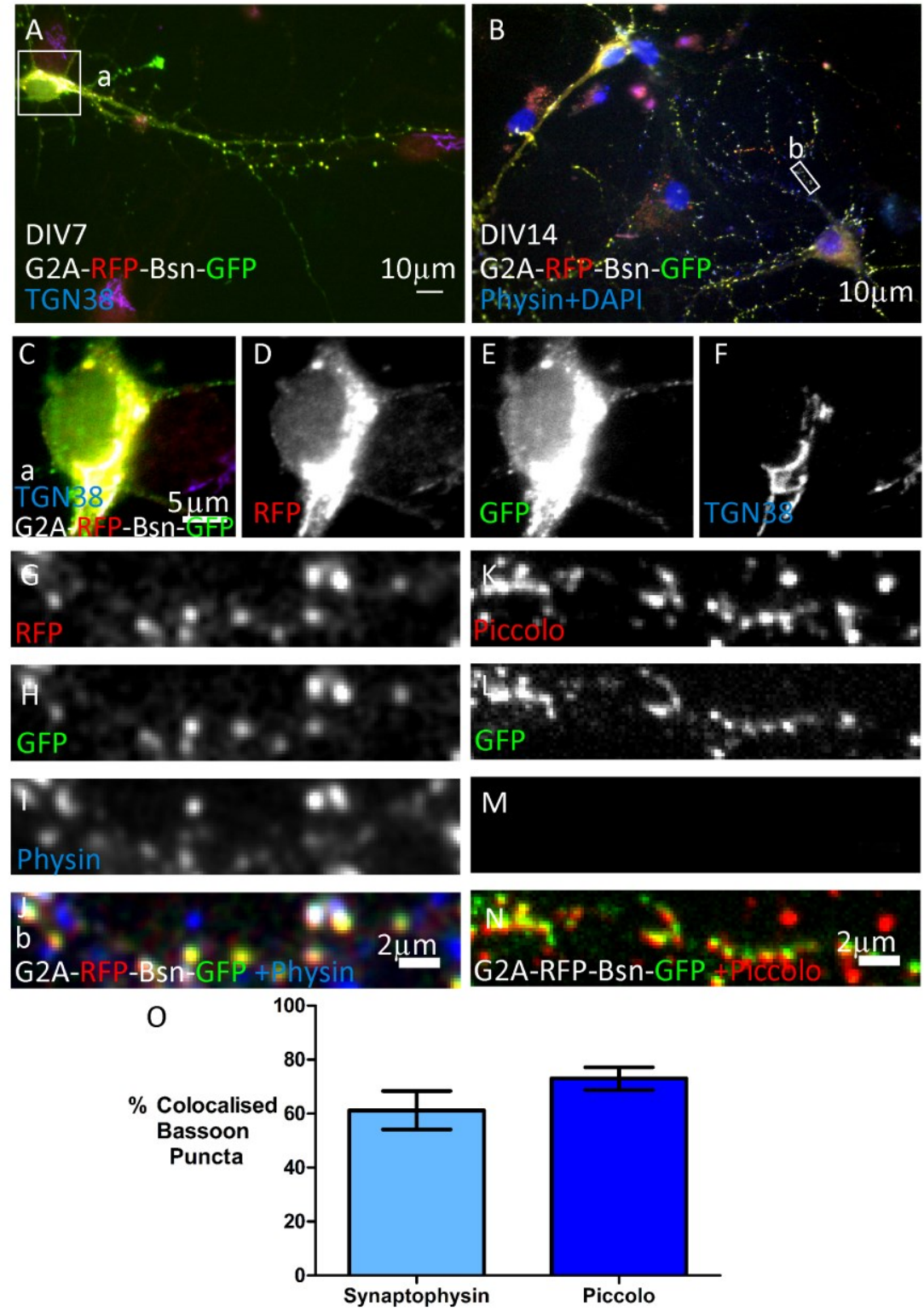

Figure 13: Mutation of the myristoyl group of Bassoon does not impede the normal Golgi and synaptic localization of the protein in young and adult neurons.

Immunostained DIV7 (A, C-F) and DIV14 (B, G-N) hippocampal neurons transfected with fulllength myristoyl mutant Bassoon constructs, post a DIV3 lipofectamine transfection, are costained with the TGN38 $(\mathbf{A}, \mathbf{C}-\mathbf{F})$, synaptophysin $(\mathbf{G}-\mathbf{J})$ and Piccolo $(\mathbf{K}-\mathbf{N})$ markers. $\mathbf{O}$ is the quantification of $(\mathbf{G}-\mathbf{N})$; data are represented as mean $\pm \mathrm{SD}, \mathrm{N}=6$ cells from two separate experiments for each quantification. Scale bars $10 \mu \mathrm{m}(\mathbf{A}-\mathbf{B}), 5 \mu \mathrm{m}(\mathbf{C}-\mathbf{F})$ and $2 \mu \mathrm{m}(\mathbf{G}-\mathbf{N})$.

The mutant fusion protein of G2A-RFP-Bsn-GFP construct localizes not unlike the full-length non-mutant constructs, in high-intensity juxtanuclear signals, 
closely localized to the TGN38 marker signal (Figure 13). This indicates that the myristoyl motif does not impair localization of Bassoon to the TGN.

The myristoyl motif also seems to permit normal trafficking and incorporation of Triton X-100 resistant recombinant Bassoon molecules into the $A Z$, as was observed by the average colocalizing Bassoon puncta population of $61.23 \% \pm$ $7.115 \%$ SD with synaptophysin and $73.03 \% \pm 4.199 \%$ SD with Piccolo (Figure 13).

This signifies that the myristoylation motif may not be involved in orchestrating the transport or the sub-cellular localization of the fusion protein into synaptic sites. The myristoyl motif may still be involved in orienting the molecule at the TGN or at the synapse. This hypothesis is explored further, later in this chapter, in section 3.5.5.

\subsection{Super resolution localization of AZPs with nanobodies}

As section 3.2 demonstrates that all second-generation full-length constructs transport and localize like endogenous Bassoon proteins, they can hence be used as tool to study the innate localization and orientation of proteins to specific nanoscopic subcellular structures in the developing neuron.

\subsubsection{Nanobody approach of the localization of tagged proteins (STED)}

In order to observe specific details of AZP localization at a higher resolution without having the labeling strategy limit the observation, I used a combination of characterized eGFP- and mRFP-tagged Bassoon constructs and GFP and RFP nanobodies.

The GFP and RFP nanobodies used are small, high-affinity, antibody-fragments created from a single amino acid chain taken from one of the two heavy chains of an alpaca IgG antibody molecule. This single amino acid chain is folded into a $\sim 10-15 \mathrm{kDa}$ epitope-binding hypervariable domain that is usually $1.5 \mathrm{~nm}$ in diameter and $2.5 \mathrm{~nm}$ in height ${ }^{81}$. These nanobodies have been routinely used to gauge interactions between tagged proteins via pulldown assays ${ }^{84}$ and for the super resolution microscopy of single-molecule localization ${ }^{82,83,85}$. The GFP and RFP nanobodies are designed such that one nanobody can identify a single, three-dimensional epitope per fluorescent protein molecule, and were bought precoupled to two molecules of bright organic Atto dyes that are suited for two-color STED microscopy. Each molecule of secondary Atto dye is $\sim 2-3 \mathrm{~nm}$ in size, thereby making the entire structure of a GFP/RFP-nanobody pre-coupled with STED dyes, maximally $9 \mathrm{~nm}$ in size in any direction. 
The eGFP or mRFP tags on Bassoon fusion products are roughly each $4 \mathrm{~nm}$ large. The fluorescent tags are boosted and visualized by the $9 \mathrm{~nm}$ nanobody complex, allowing the localization of Bassoon molecules to be within a maximal $13 \mathrm{~nm}$ distance from the Bassoon epitope. This labeling protocol allows signals to be localized roughly three times closer to the molecule of interest, than with the application of antibodies. Additionally, it also improves the labeling frequency because large poly- and monoclonal antibody clusters inhibit labeling of epitopes within $30 \mathrm{~nm}$ of any already labeled epitope ${ }^{94}$. To make the best use of these optimally labeled constructs, a two-color STED setup that regularly obtained a resolution of 30nm for Atto 594 channel and for 20nm for the Atto kk1212/647 channel was used ${ }^{79}$. As every tagged Bassoon molecule is boosted with one nanobody complex, which generates a signal size smaller than of the $20 \mathrm{~nm}$ resolution limit of the STED microscope, all signals $\geq 20 \mathrm{~nm}$ can be assumed to represent the $x y$ localization coordinates of the tagged Bassoon epitope (Figure 14).

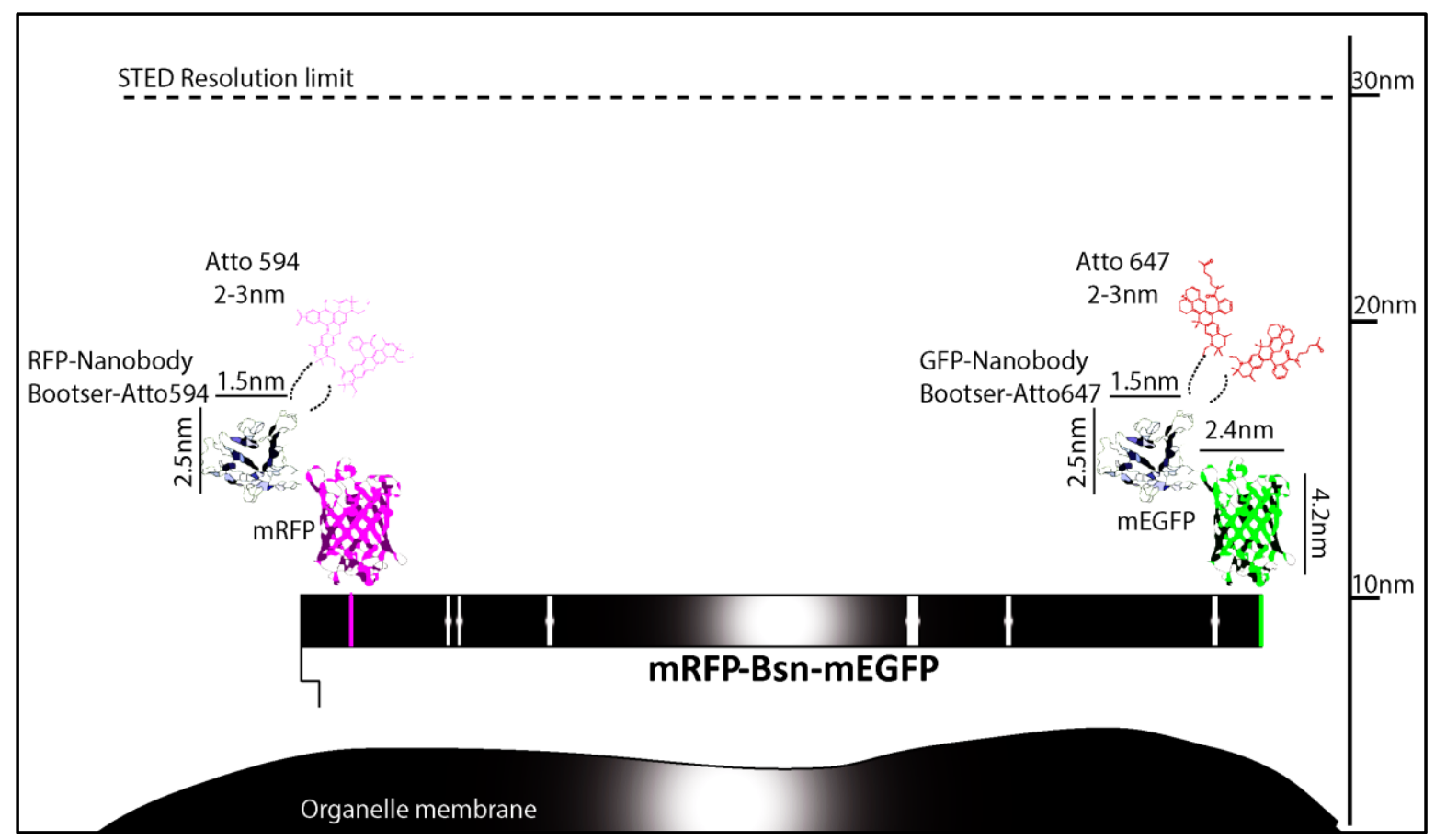

Figure 14: Using GFP and RFP specific nanobodies to visualize of single molecules of tagged Bassoon protein under $30 \mathrm{~nm}$ resolution. GFP and RFP tags localize $\sim 4 \mathrm{~nm}$ from the $\mathrm{N}$ and/or Cterminus of the Bassoon molecule and are visualized on STED scopes using specific 3-D domain binding GFP and/or RFP nanobodies (2.5 nm in diameter) that come pre-coupled to two molecules of $\sim 2-3 \mathrm{~nm}$ in size Atto dyes. The entire tag-nanobody complex is $\sim 13 \mathrm{~nm}$ in size and significantly below the resolution of two-color STED scopes used in this study. 


\subsubsection{Localization of tagged AZPs at their respective sub-compartments (STED)}

To visualize the localization of tagged AZP proteins Munc13-1 and Bassoon, I transfected their most well characterized constructs, full-length eGFP-Munc13$1^{100-102}$ and recombinant eGFP-95-Bassoon ${ }^{30,70,99,47}$. Both constructs have an Nterminally tagged GFP that was boosted with the GFP nanobody pre-coupled to Atto 647 dyes. The AZPs were visualized with two-color STED microscopy at their respective Golgi compartment labeled with traditional antibodies for TGN38 and GM130, respectively.

With the increase in resolution the amount of visual colocalization of AZPs to the Golgi markers decreases, although $42.2 \% \pm 14.9 \%$ SD of the total Munc13-1 signals colocalize to the cis-Golgi marker and $36.2 \% \pm 12.9 \%$ SD of 95 -Bassoon colocalizes with trans-Golgi marker. Additionally all the signals in the 95-Bassoon and Munc13-1 transfected images were recorded and their populations grouped in two distance categories from the Golgi signals. A 0-100nm distance range from Golgi signals represents signals at the Golgi lamella, as typically the diameter of a single Golgi marker signal was observed to be around $80 \mathrm{~nm}$ and an extra $+20 \mathrm{~nm}$ allowance was added to the range as the TGN structure is a large three-dimensional structure, which may be surrounded by out of focus signals neighboring the focal plane imaged. A $101 \mathrm{~nm}-1 \mu \mathrm{m}$ distance range from Golgi signals represents AZP signals between and around the TGN lamella. For both 95-Bassoon (fraction of total population: 0.41 at lamella and 0.59 away from lamella) and Munc13-1 (fraction of total population: 0.39 at lamella and 0.61 away from lamella) there is a significantly higher population of signals distributed away from the Golgi lamella than at the lamella. This could signify a large population of signals, which were previously assumed to be colocalized with confocal microscopy, to be AZPs loaded on to transport carriers in transit to or from the Golgi compartments (Figure 15). 


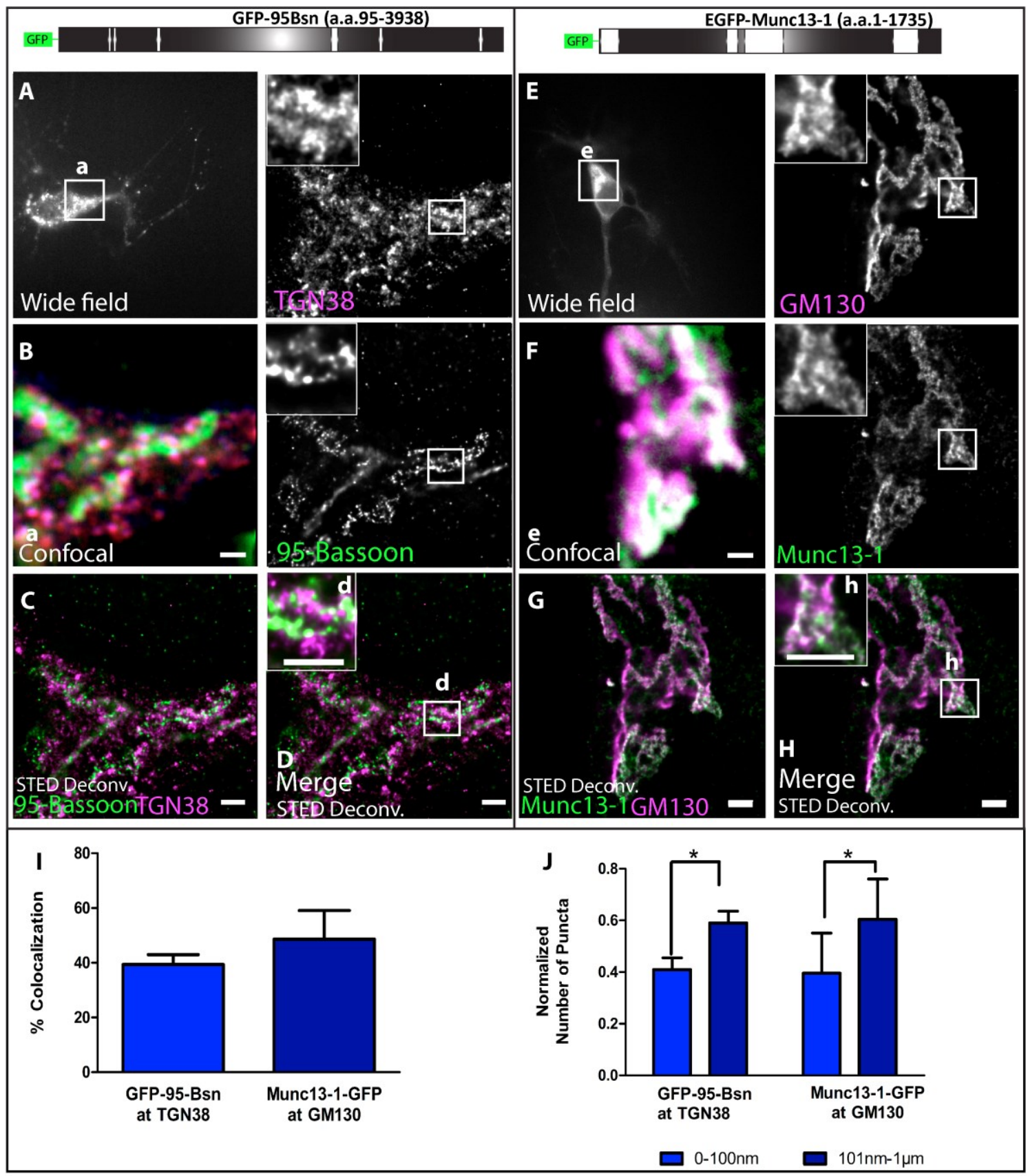

Figure 15: Super resolution localization of GFP tagged Bassoon (95-3938) and full-length Munc13-1 at their respective Golgi sub-compartments.

A and E, wide field over view of transfected DIV7 neurons immunostained for recombinant Bassoon and Munc13-1 using the GFP nanobody with TGN38 or GM130 antibodies, respectively. $\mathbf{B}$ and $\mathbf{F}$, represent blow-ups of $\mathbf{a}$ and $\mathbf{e}$ ROIs, which were imaged first with confocal and then twocolor STED microscopy, $\mathbf{C}-\mathbf{D}$ and $\mathbf{G}-\mathbf{H}$, respectively. STED zooms of Bassoon (d) and Munc13-1(i). I, colocalization quantification and $\mathbf{J}$ distribution quantification at and away from Golgi lamella i.e. $0-100 \mathrm{~nm}$ or $101 \mathrm{~nm}-1 \mu \mathrm{m}$, respectively. Data represented as mean $\pm \mathrm{SD}$, $\mathrm{N}=10$ cells from two separate experiment, ${ }^{*} p<0.05$. Scale bars $1 \mu \mathrm{m}$ (B-E). 


\subsubsection{Localization of full-length Bassoon construct at neighboring Golgi structures}

Low colocalizations of recombinant Munc13-1 and 95-Bassoon signals were seen at their respective cis-and trans-Golgi network lamella at a $20 \mathrm{~nm}$ resolution limit (Figure 15). This result suggests that visualizing these AZPs at super-resolutions might reveal clearer picture of their nanoscopic localization and their low colocalizations might actually reflect their localization to a different neighboring Golgi compartment than previous assumed. Additionally, the use of traditional antibody complexes to label the Golgi markers may boost the out of focus Golgi lamella signals, presenting the nanobody labeled AZPs at the wrong Golgi localization. To test whether with an increase in resolution it was possible to visualize AZPs at neighboring Golgi compartments and to employ nanobodies in both STED channels, I transfected a RFP tagged full-length Bassoon construct and CFP-Golgi construct, and visualized them using the RFP and GFP nanobody in the somas of young (DIV6) neurons.

The CFP-Golgi construct targets 81 amino acids of the $\mathrm{N}$-terminal cytoplasmic domain of the $\beta-1,4$-galactosyltransferase 1 transmembrane protein that localizes specifically in the lamellae of the trans-Golgi compartment ${ }^{104}$. In contrast the TGN38 protein is an integral membrane protein of the trans-Golgi network lamellae and is retained by a different mechanism into the TGN from that of the transferase enzymes of the trans-Golgi ${ }^{105}$. To visualize the difference in localization of the trans-Golgi compartment and the trans-Golgi network compartment with two-color STED, the TGN38 antibody was labeled in CFP. Golgi transfected neurons and revealed no colocalization upon visual inspection, signifying the presence of two neighboring Golgi compartments occupying different $z$-position localizations in the soma. In order to ascertain the specific localization of full-length Bassoon to the two neighboring Golgi compartments, the tagged molecule was visualized in CFP-Golgi-transfected and TGN38-labeled neurons.

Surprisingly, there is a significantly higher yellow visual colocalization signal of the mRFP-Bsn construct to the TGN38 lamella, compared to almost no colocalization seen with the CFP-Golgi construct (Figure 16). This result illustrates that Bassoon is specifically localized only to the trans-Golgi network compartment and that irrespective of the use of nanobodies or antibodies to label the Golgi sub-compartments, high colocalization of an AZP can be detected at its expected localization.

This result raises a discrepancy regarding the low colocalization of the 95Bassoon recombinant protein observed at the TGN38 marker, while full-length mRFP-Bsn recombinant protein displays a high colocalization. This difference in colocalization of the same AZP molecules, in constructs that both reach synaptic sites, signifies a role of the missing 94 amino acids of Bassoon's $\mathrm{N}$-terminus in 
its proper localization, orientation and hence consequently its transport on correct transport precursors to synapse. This topic is explored in detail in section 3.5.5.

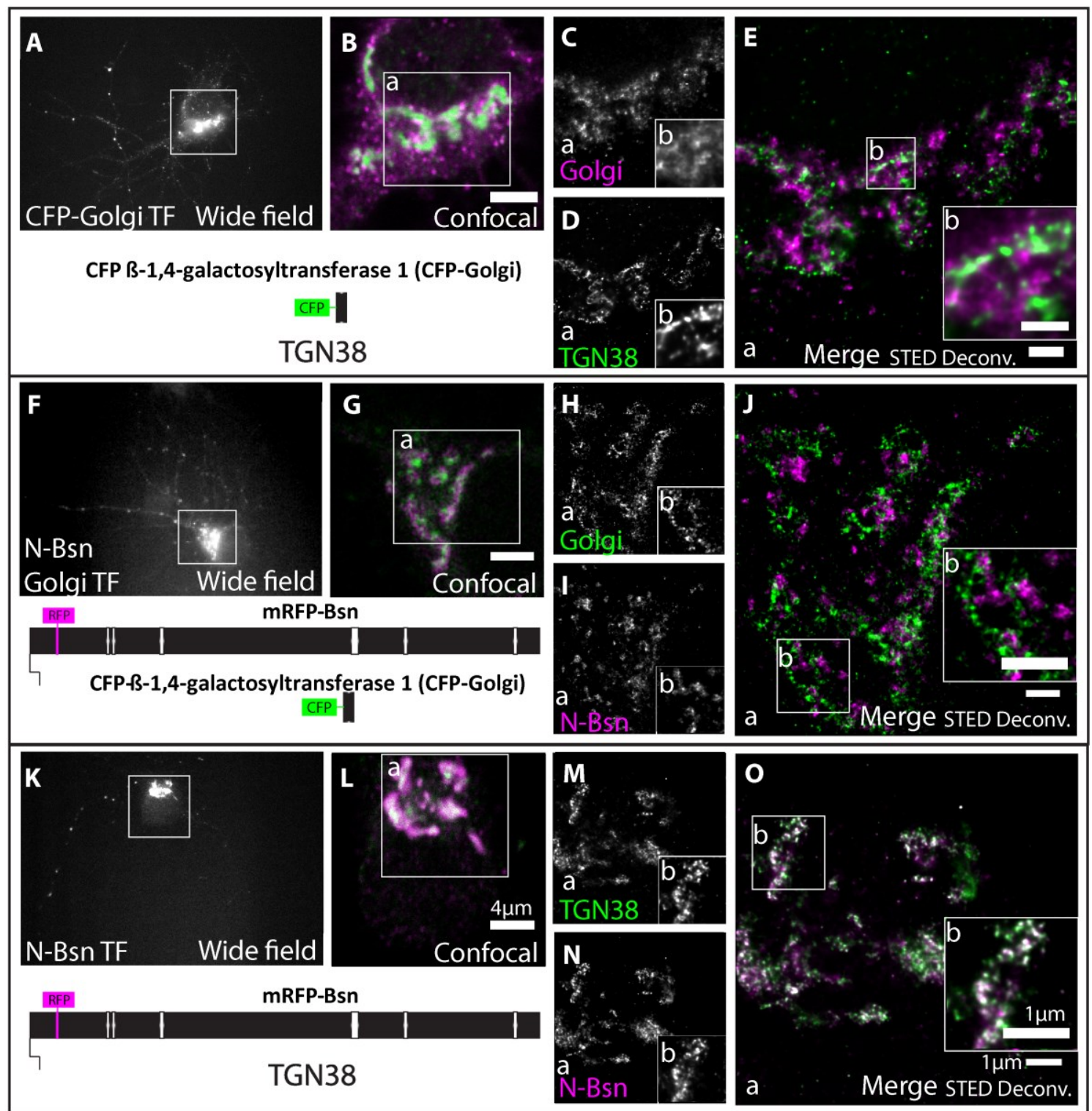

Figure 16: Full-length Bassoon localizes specifically to the trans-Golgi network compartment instead of the trans-Golgi sub-compartment. DIV7 hippocampal neurons were transfected with CFP-Golgi (trans-Golgi sub-compartment maker), full-length single-tagged mRFP-Bsn construct, and immunostained using GFP and/or RFP nanobodies against tagged constructs and from A-E with TGN38 (trans-Golgi network maker). Two-color STED images of both Golgi subcompartment markers (A-E), CFP-Golgi and RFP-Bsn constructs (F-J), and RFP-Bsn at TGN38 (K-O). A, F, K show wide field over view of transfected construct, $\mathbf{B}, \mathbf{G}, \mathbf{L}$ the confocal zoomed image of the soma, inset a reflects both the single channels and merged STED deconvolved (Deconv.) images of $\mathbf{C}-\mathbf{E}, \mathbf{H}-\mathbf{J}$, and $\mathbf{M}-\mathbf{O}$, and $\mathbf{b}$ the STED zooms. With the images in each panel, a schematic diagram of the transfected constructs used is supplied. Scale bars $4 \mu \mathrm{m}(\mathbf{B}, \mathbf{G}$ and $\mathbf{L})$ and $1 \mu \mathrm{m}(\mathbf{E}, \mathbf{J}$ and $\mathbf{O})$. 


\section{Orientation and organization of the Bassoon molecule at the trans-Golgi network, on transport vesicles and at synaptic sites}

It has already been shown that endogenous AZP Bassoon signals are best visualized at $37^{\circ} \mathrm{C}$ (Figure 3 and Figure 4), at and around the trans-Golgi network lamella, and are predominantly $96-150 \mathrm{~nm}$ in size at their juxtanuclear localization (Figure 5). These endogenous Bassoon signals are uniformly distributed along the axons of young neurons (Figure 7), and roughly $30 \%$ of their population was observed co-trafficking with other AZPs (Figure 8).

Additionally, full-length tagged Bassoon constructs, characterized to reflect endogenous Bassoon localizations (Figure 10-Figure 12).

In comparison, the well-characterized first-generation GFP-95-Basoon construct, despite also being observed colocalizing with a subpopulation of dense-core vesicles positive for the Syt4 marker in the soma (Figure 6), has a significantly lower colocalization to the TGN lamella (Figure 9). This recombinant protein was also shown to be loaded on to a large number of clear-core vesicles at juxtanuclear positions (Dresbach and Wittenmayer, unpublished).

These results raise interesting questions about the orientation and localization of the Bassoon molecule on its journey to the synapse and how deletion mutants of Bassoon alter these processes that may ultimately influence $A Z$ assembly.

To address these topics, I will in this section study the following:

1) the orientation of single- and double-tagged full-length Bassoon constructs at TGN markers with STED microscopy;

2) the orientations of a range of Bassoon mutants at the TGN with STED microscopy;

3) the orientation of single-tagged Bassoon molecules on transport vesicles

4) the organization of Bassoon molecules at the TGN with FLIM;

5) the organization of Bassoon molecules at synaptic sites with FLIM;

6) a comparison of the organization of Bassoon molecules as they move from the TGN, on transport vesicles, and at the synapse.

Together, these questions will help us visualize the proper localization, orientation and organization of an essential AZ backbone protein Bassoon and will reveal how AZP molecules are organized before and after AZ assembly. 


\subsection{Localization of full-length Bassoon constructs to the trans-Golgi network}

The orientation of Bassoon at synapses has been previously shown via stochastic optical reconstruction microscopy (STORM) and electron microscopy (EM), where the $\mathrm{C}$ - terminus of the Bassoon molecule faces the presynaptic membrane and its $\mathrm{N}$ - terminus faces the away from the presynaptic membrane and lies roughly $50 \mathrm{~nm}$ from the $\mathrm{C}$-terminus ${ }^{52,76}$. The precise orientation of Bassoon has not as yet been studied at another substructure; hence, to begin with, this subsection will delve into Bassoon's orientation at the TGN.

The trans-Golgi network (TGN) is a tubular network of lamellae that forms a separate subcellular compartment in the soma of neurons. The TGN usually follows the trans-Golgi compartment and is a major station for the recruitment, sorting, and transport of proteins to various subcellular destinations ${ }^{106}$. TGN38 is a specific type I, integral membrane protein found in the TGN lamella. TGN38 is known to form heterodimers with TGN46, which in turn promotes constitutive recycling of TGN38 positive transporters and its cargo, back and forth from the TGN to plasma membrane. TGN38 is a 268 amino acid (a.a.)-long protein that possesses a luminal domain at its $\mathrm{N}$-terminus, followed by a 21 a.a. hydrophobic transmembrane region and a 33 a.a-long C-terminal tail that contains the TGN targeting motif ${ }^{105}$. The TGN38 marker used in this section recognizes a large portion (31-244 amino acids) of the TGN protein including its transmembrane domain and has been previously used in the field for targeting and localization experiments ${ }^{30,32,70}$. Full-length single- and double-tagged Bassoon constructs that possess either a mEGFP and/or a mRFP tag at either termini, which have been characterized in Figure 10-Figure 12, will be observed at the TGN38 lamella.

\subsubsection{Orientation of full-length double-tagged construct at TGN38}

To visualize both termini of a tagged full-length Bassoon molecule, the doubletagged mRFP-Bsn-mEGFP construct was used. The termini of the construct were visualized by employing either the RFP nanobody or the GFP nanobody at the TGN38 marker and imaged using a two-color STED setup. The DIV7 transfected neurons were found and first imaged in the wide field mode to gauge the quality of transfection. A $20 \mu \mathrm{mX} 20 \mu \mathrm{m}$ area containing the soma of the transfected neurons is focused and scanned in the confocal mode, at the z-plane containing the highest TGN38 signal, and a $10 \mu \mathrm{m} \times 10 \mu \mathrm{m}$ area within the confocal image was then scanned for both channels in the STED mode. This standard imaging process is applied for the acquisition of all STED images in this section. 
STED imaging reveals a striking visual of $\mathrm{N}$-terminal Bassoon signals colocalizing to the TGN38 signals (seen as white Bassoon punctate signals), and C-terminal Bassoon signals predominantly appear to be non-colocalized signals close to the TGN38 signals (seen as green Bassoon punctate signals). The C-terminal Bassoon signals appear to be high-intensity signals that are in focus with the TGN38 signals, although their localization appears to be next to rather than on the TGN38 signals. Further inspection into the population of colocalizing Bassoon signals demonstrates the presence of a significantly higher number of colocalizing N-terminal Bassoon signals/image (67.5\% $\pm 12.5 \% \mathrm{SD})$, in comparison to the $29.8 \%$ ( $\pm 7.7 \%$ SD) of C-terminal Bassoon signals/image that appear colocalized with the TGN38 marker.

This suggests that the colocalizing N-terminal Bassoon signals and the TGN38 signals are located within the $20 \mathrm{~nm}$ resolution limit of the STED microscope and that the large fraction of non-colocalizing C-terminal Bassoon signals are not.

To gather a detailed understanding of the localization of $\mathrm{N}$ - and C-terminal Bassoon signals, an analysis of the distribution pattern of the Bassoon signals was conducted and sorted in two distance categories: a) 0-100nm (representing signals that are at or close to the 80nm TGN38 lamella signals) and b) 101nm$1 \mu \mathrm{m}$ distance category (representing all signals that are between and around TGN38 lamella). Signals further than $1 \mu \mathrm{m}$ in distance from the TGN38 lamella, in any direction, were excluded from the analyses and considered to be AZP molecules that were not being oriented at the trans-Golgi network.

The analysis reveals a significantly higher fraction of $\mathrm{N}$-terminal Bassoon signals located on average $32.5 \mathrm{~nm} \pm 8 \mathrm{~nm}$ from the TGN lamella, within the distance category of $0-100 \mathrm{~nm}(0.67 \pm 0.1 \mathrm{SD})$, in comparison to its signals in the $101 \mathrm{~nm}-1 \mu \mathrm{m}$ distance category $(0.33 \pm 0.1 \mathrm{SD})$ which are located on average at $210.6 \mathrm{~nm} \pm 13 \mathrm{~nm}$. An opposite trend is observed for the C-terminal Bassoon signals, which display significantly higher signal fractions residing within the distance category of $101 \mathrm{~nm}-1 \mu \mathrm{m}(0.63 \pm 0.1 \mathrm{SD})$ at an average distance of $229.4 \mathrm{~nm} \pm 5.3 \mathrm{~nm}$, rather than the $0-100 \mathrm{~nm}$ category $(0.37 \pm 0.1 \mathrm{SD})$ that localizes on average $60.4 \mathrm{~nm} \pm 7 \mathrm{~nm}$ from the TGN lamella (Figure 17 and Appendix $\mathrm{G}$ for average distances).

These results demonstrate that the $\mathrm{N}$-termini of Bassoon molecules colocalize with the TGN38 signals and are localized below the $20 \mathrm{~nm}$ resolution limit to be observed as such, and are distributed within $0-100 \mathrm{~nm}$ of the TGN38 signals, while the C-terminus of the Bassoon molecules are localized close to TGN38 signals and are distributed within the distance range of $101 \mathrm{~nm}-1 \mu \mathrm{m}$. 


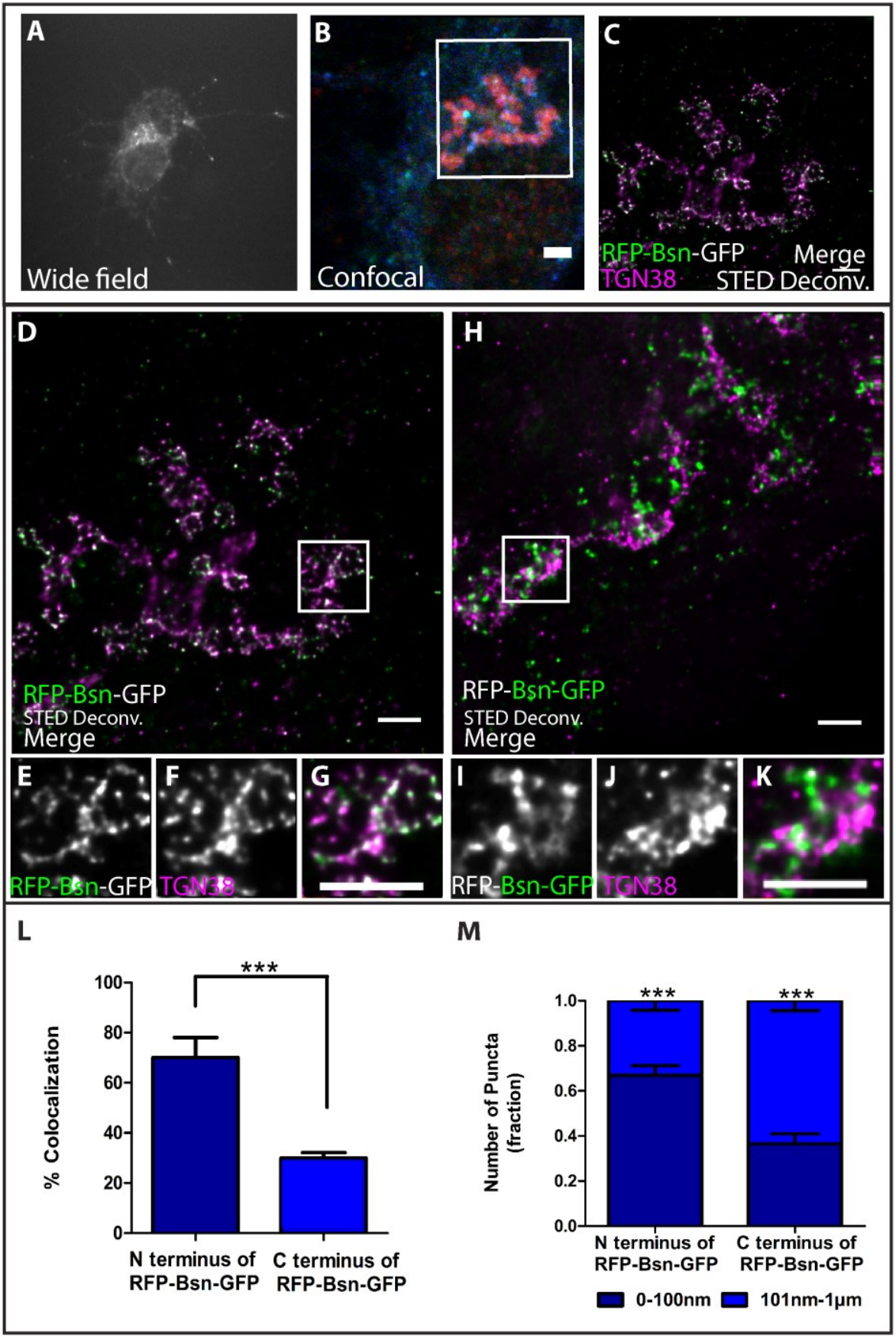

Figure 17: Orientation of mRFP-Bsn-mEGFP at the trans-Golgi network. DIV7 hippocampal neurons transfected with double-tagged full-length Bassoon, immunostained with the trans-Golgi network marker TGN38 and Bassoon's N-terminus using a RFP-nanobody-Atto594 (A-G) or its C-terminus using a GFP-nanobody-Atto594 (H-K). The experimental schematic demonstrating the acquisition of two-color STED images begins with the wide field over view of the transfected neuron $(\mathbf{A})$, its corresponding confocal over view of the soma, with GFP autofluorescence in blue, (B) and a $10 \mu \mathrm{m} \times 10 \mu \mathrm{m}$ inset scanned in STED mode to visualize the construct's $\mathrm{N}$-terminus (C, D) and C-terminus (H). Zoomed split and merged channels of $\mathbf{E}-\mathbf{G}$ and $\mathbf{I}-\mathbf{K}$, are representations of the insets in the STED images $\mathbf{D}$ and $\mathbf{H}$ respectively. $\mathbf{L}$ represents the colocalization quantification, and $\mathbf{M}$ represents the distribution quantification at and away from TGN lamella i.e. $0-100 \mathrm{~nm}$ or $101 \mathrm{~nm}-1 \mu \mathrm{m}$, respectively. Data are represented as mean \pm SD, $\mathrm{N}=10$ cells from two separate experiments, statistically tested with a one-way annova with the Tukey's multiple comparison's post-hoc test ${ }^{\star \star *} p \leq 0.001$. Scale bars $2 \mu \mathrm{m}(\mathbf{B})$ and $1 \mu \mathrm{m}(\mathbf{C}-\mathbf{K})$. 


\subsubsection{Orientation of full-length single-tagged constructs at TGN38}

To verify whether the introduction of fluorescent tags on both the termini of all transfected Bassoon molecules creates an artificially exaggerated orientation of the molecule at TGN, single-tagged Bassoon constructs were transfected and imaged, following the standard protocol used for Figures 10 and Figure 16.

In addition to rule out the possibility of varying avidities of the RFP and GFP nanobodies influencing the orientation results obtained from the mRFP-BsnmEGFP transfections (Figure 17), RFP-tagged full-length mRFP-Bsn and BsnmRFP constructs were compared.

The N-termini of Bassoon visualized by the RFP tag of mRFP-Bsn is observed as white, punctate, colocalized signals that have a significantly higher population of signals colocalizing to the TGN38 $(84.8 \% \pm 4.6 \% \mathrm{SD})$, in contrast to the large population of non-colocalizing Bsn-mRFP signals present. Although $44.4 \% \pm$ $2.4 \%$ SD population of the all Bsn-mRFP signals were seen to colocalize with TGN38 signals and were distributed within $0-100 \mathrm{~nm}$ distance category at an average distance of $50.3 \mathrm{~nm} \pm 3 \mathrm{~nm}$ from the TGN lamella. These signals represented only a 0.45 faction $\pm 0.03 \mathrm{SD}$ of the total signals/image. The majority of the Bsn-mRFP signals, were in close proximity but not at the TGN38, was distributed within the $101 \mathrm{~nm}-1 \mu \mathrm{m}$ distance category at an average distance of $192 \mathrm{~nm} \pm 11.6 \mathrm{~nm}$ from the TGN38 and represented the 0.55 fraction ( $\pm 0.03 \mathrm{SD}$ ) of total population of its signals. The inverse observation is apparent for the highly colocalized mRFP-Bsn signals that are predominantly distributed within the 0 $100 \mathrm{~nm}$ distance category at an average distance of $30.5 \mathrm{~nm} \pm 6.9 \mathrm{~nm}$ from the TGN lamella, representing the 0.84 fraction $\pm 0.04 \mathrm{SD}$ of the total signal population. While the significantly lower fraction of mRFP-Bsn signals are distributed within $101 \mathrm{~nm}-1 \mu \mathrm{m}$ distance category at an average distance of $154 \mathrm{~nm} \pm 12 \mathrm{~nm}$ from the TGN, representing the population fraction size of $0.15 \pm$ 0.04 SD (Figure 18 and Appendix G).

These results are similar to those obtained from the double-tagged Bassoon constructs and show that despite the number and type of tag used the N-termini of Bassoon molecules are largely colocalized and oriented towards the TGN38 lamella while C-termini of Bassoon are mainly localized close to the TGN38 and appear to be oriented away from the TGN38 lamella. 
Figure 18: Orientation of mRFP tagged full-length Bassoon constructs at the trans-Golgi network (TGN).Transfected DIV7 hippocampal neurons immunostained for the either termini of full-length Bassoon with RFP-nanobody-Atto594 and the TGN38 marker. Two-color deconvolved 10 $\mu \mathrm{m} \mathrm{X}$ $10 \mu \mathrm{m}$ STED images of the N-terminus of Bassoon (A) and the blow-ups of its inset (B-D) or the C-terminus of Bassoon $(\mathbf{A})$ and the blow-ups of its inset $(\mathbf{F}-\mathbf{H})$. I represents the colocalization quantification, and $\mathbf{J}$ represents the distribution quantification at and away from TGN38 lamella i.e. $0-100 \mathrm{~nm}$ or $101 \mathrm{~nm}-1 \mu \mathrm{m}$, respectively. Data are represented as mean $\pm S D, N=10$ cells from two separate experiment, statistically tested with a one-way annova with the Tukey's multiple comparison's post-hoc test ${ }^{*} p<0.05$ and ${ }^{* * *} p \leq 0.001$. Scale bars $1 \mu \mathrm{m}(\mathbf{A}-\mathbf{H})$.

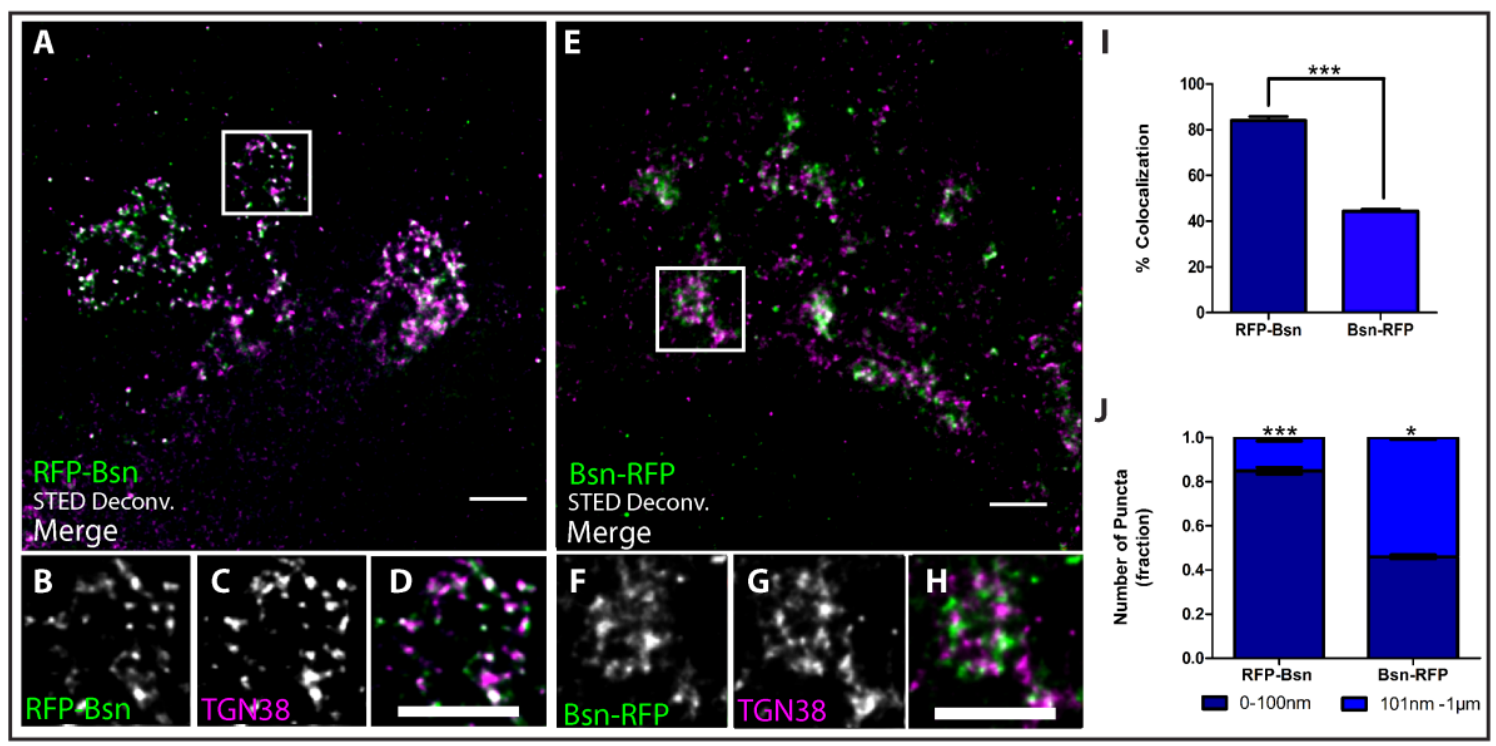

\subsubsection{Orientation of full-length single and double-tagged constructs at Syn6}

To ascertain whether the orientation of Bassoon is specifically regulated to the TGN38 domain or whether the orientation of Bassoon is uniform all over the TGN lamella, the orientation of double-tagged and single-tagged full-length Bassoon constructs were visualized at another TGN lamella protein; Syntaxin6 (Syn6),

Syn6 much like TGN38 is a protein present at the lamellae of the trans-Golgi network that is involved in sorting and trafficking of proteins from the TGN to the plasma membrane and back. Syn6 is a 255 a.a.-long protein that possesses a Nterminal soluble SNARE domain that localizes Syn6 to the TGN, and possess an additional sorting motif that promotes retrograde transport of the Syn6 positive vesicles. It also possesses a hydrophobic C-terminal transmembrane region that acts as a membrane anchor and regulates the sorting of proteins at the TGN lamella ${ }^{107,108}$. The antibody toward Syn6 targets an epitope spanning 6-136 amino acids that contains its $\mathrm{N}$-terminal TGN-targeting motif and therefore works as an additional marker to label the TGN lamella. Images of the single- and double-tagged Bassoon constructs were imaged and processed with the standard STED imaging protocol. 
Remarkably, the N-terminally tagged Bassoon signals of both the mRFP-BsnmEGFP and mRFP-Bsn construct have high colocalizations with the Syn6 signals at $71.8 \%$ and $75.8 \%$ respectively, while the C-termini of the mRFP-Bsn-mEGFP $(23.8 \%)$ and Bsn-mRFP (43.7\%) construct have significantly lower number of colocalized signals.

These results reflect the same colocalization patterns as those observed in the TGN38 images and therefore expectedly present with similar signal distribution patterns.

Wherein, significantly higher fraction of signals were distributed within $0-100 \mathrm{~nm}$ distance category for the $\mathrm{N}$-termini of single- $(0.8$ population fraction $\pm 0.03 \mathrm{SD})$ and double-tagged Bassoon molecules ( 0.71 population fraction $\pm 0.02 S D$ ), while the major fraction of C-terminal Bassoon signals from the single- (0.62 population fraction $\pm 0.03 \mathrm{SD}$ ) and double-tagged (0.65 population fraction $\pm 0.04 \mathrm{SD})$ images lay in the $101 \mathrm{~nm}-1 \mu \mathrm{m}$ category (Figure 19).

This result reflects that Bassoon molecules are orientated robustly all over the TGN lamella with their N-termini localizing within $0-100 \mathrm{~nm}$ of the TGN lamella, irrespective of the application of either TGN38 or Sny6 marker, and are present as highly colocalized signals even after being resolved by STED. The C-termini of tagged Bassoon molecules are also always seen localized close to the TGN lamella, irrespective of the marker used, and are present as clearly resolved signals that are majorly distributed within the $101 \mathrm{~nm}-1 \mu \mathrm{m}$ distance category. 


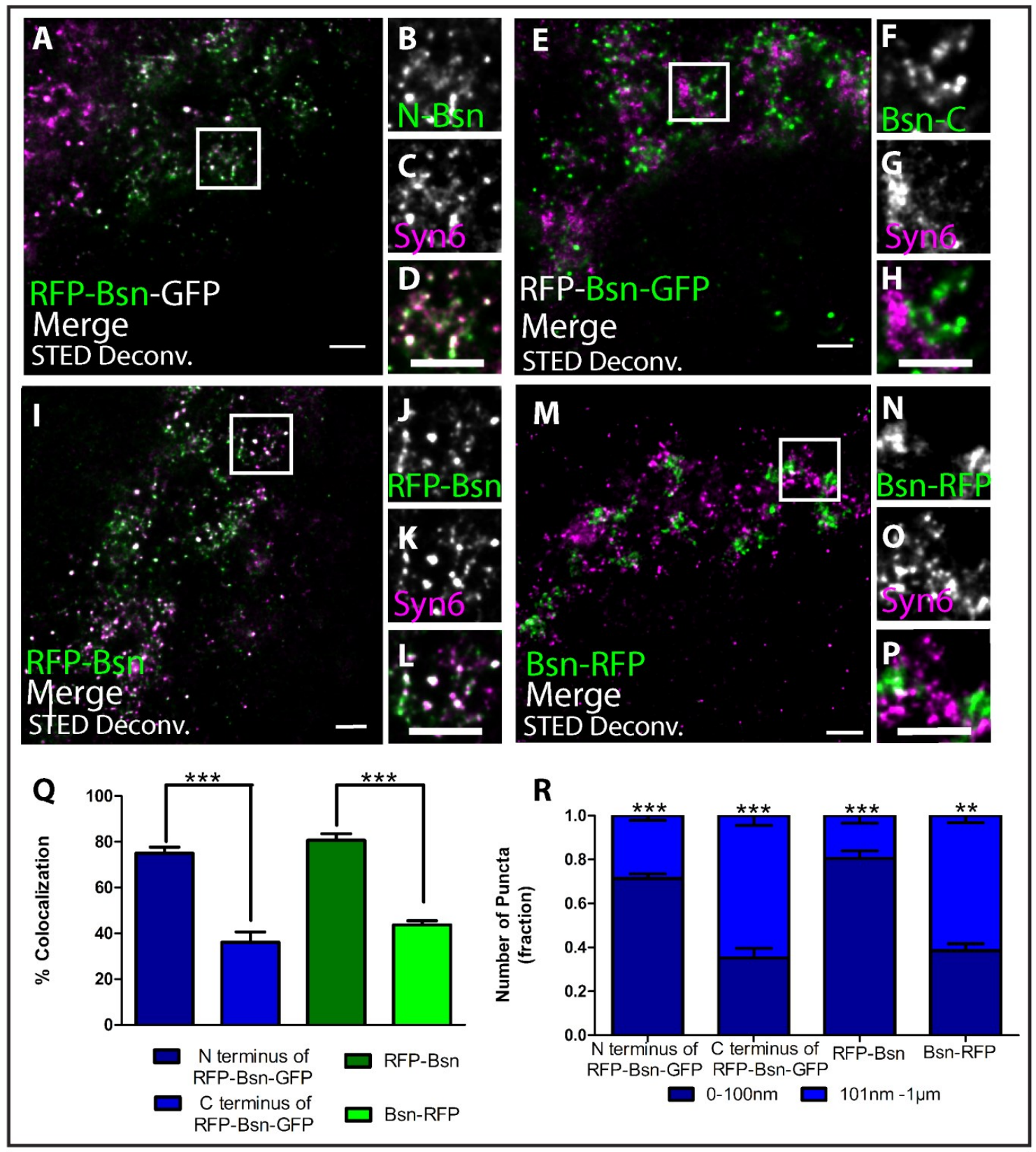

Figure 19: Orientation of single and double-tagged full-length Bassoon constructs at the transGolgi network marker: Syntaxin 6 (Syn6).

Transfected DIV7 hippocampal neurons immunostained for the either or both termini of full-length Bassoon with RFP-nanobody-Atto594 and/or GFP-nanobody-Atto647 with the Syn6 marker. Twocolor, deconvolved, $10 \mu \mathrm{m} \times 10 \mu \mathrm{m}$ STED images and their insets, representing the $\mathrm{N}$-terminus of double-tagged and single-tagged Bassoon constructs in panels $\mathbf{A}-\mathbf{D}$ and $\mathbf{L}-\mathbf{L}$, respectively. Similar STED images of the C-terminus of the double-tagged and the single-tagged Bassoon constructs, are available in panels $\mathbf{E}-\mathbf{H}$ and $\mathbf{M}-\mathbf{P}$, respectively. Graph $\mathbf{Q}$ quantifies the amount the colocalization and graph $\mathbf{R}$ quantifies the signal distributions at and away from TGN38 lamella i.e. $0-100 \mathrm{~nm}$ or $101 \mathrm{~nm}-1 \mu \mathrm{m}$, respectively. Data are represented as mean $\pm \mathrm{SD}, \mathrm{N}=10$ cells from two separate experiment, statistically tested with a one-way annova with the Tukey's multiple comparison's post-hoc test ${ }^{* *} p \leq 0.01$ and ${ }^{* * *} p \leq 0.001$. Scale bars $1 \mu \mathrm{m}(\mathbf{A}-\mathbf{P})$. 


\subsubsection{Detailed orientation of Bassoon molecule at the trans-Golgi network}

STED reveals that $\mathrm{N}$-terminal full-length Bassoon signals colocalize within the $20 \mathrm{~nm}$ resolution limit of the setup, and shows the significantly higher populations of its signals distributed within the $0-100 \mathrm{~nm}$ distance range. To repeat this novel observation with another high-resolution microscopy technique and potentially to reveal new insights about the detailed localization of the $\mathrm{N}$-terminus of Bassoon to the TGN lamella, I performed fluorescence-lifetime imaging microscopy (FLIM).

This imaging technique can be used to display interactions of tagged proteins within $0-5 \mathrm{~nm}$ of each other. Young mRFP-Bsn expressing hippocampal neurons were stained with the TGN38-Alexa488 and fluorescence lifetime of Alexa488 was measured in order to determine FRET with RFP-Bsn. FLIM recordings were imaged in the soma at a focal plane presenting the highest intensity signals of the TGN38 marker. The TGN38 labeled Alexa488 lifetime in transfected neurons were compared to the Alexa488 lifetimes of the TGN38 marker in untransfected somas of neurons, from the same culture and coverslip.

A lifetime of 1.75 ns was recorded at TGN38 positive structures, which were represented by a yellow to red color (Figure $19 \mathrm{~F}$ ), in untransfected neuronal somas. The N-terminus of mRFP-Bsn is labeled using the RFP-nanobodyAtto594 and the RFP nanobody is expected to FRET with the Alexa488 fluorophore, when both dyes are present within a $5 \mathrm{~nm}$ distance of each other. A FRET interaction is reflected by lower lifetimes in nanoseconds and is represented by a cooler look up table (LUT) color.

The lifetime of TGN38 in the presence of the N-terminally tagged RFP molecules of full-length mRFP-Bsn, is 1.7ns and insignificantly different from the TGN38 lifetimes in untransfected neurons. This is also confirmed by visualizing the yellowish-red LUT color for the transfected and its neighboring untransfected soma. This implies that the RFP tag stained with RFP-nanobody-Atto594 is not within $5 \mathrm{~nm}$ of the Alexa488 dye, and by relation, the RFP-tag at the $97^{\text {th }}$ a.a. of Bassoon and the TGN38 antibody epitope site do not directly interact (Figure 20).

Additionally this result shows that the colocalizing signals of the RFP tag at the Nterminus of Bassoon, at the TGN38 signals, seen within the $20 \mathrm{~nm}$ resolution of STED are in fact localized between 6 and 20nm from each other. More significantly it highlights that as Bassoon is oriented with its $\mathrm{N}$-terminus facing the TGN38, the first few amino acids in the $\mathrm{N}$ terminus of Bassoon must be involved in its proper localization and orientation to the TGN. An interesting candidate that might specifically influence this interaction is the hydrophobic myristoyl group present at the $2^{\text {nd }}$ amino acid of Bassoon. Myristoyl mutants as well as mutants lack either one of both termini of Bassoon may present alternative orientation of 
the Bassoon molecule at the TGN. This will be investigated in detail the next subsection (3.5.5).

Figure 20: FLIM imaging of N-terminus of Bassoon to TGN38 to visualize interaction within $5 \mathrm{~nm}$. A-F and G-L, represent DIV7 untransfected and transfected mRFP-Bsn hippocampal neurons labeled with the RFP-nanobody-Atto594 to visualize the construct and TGN38 visualized and boosted with an Alexa488 dye, respectively. A-C and $\mathbf{G}-$ I represent the immunolabeling of the samples and $\mathbf{D}-\mathbf{F}$ and $\mathbf{J}-\mathbf{L}$ represent the Alexa intensity and lifetime information. Insets of $\mathbf{C}$ and I were imaged for lifetimes in $\mathbf{D}-\mathbf{F}$ and $\mathbf{J}-\mathbf{L}$, respectively. $\mathrm{N}=4$ from two cultures. LUT lifetimes range from 1.5 to 2.0 ns.

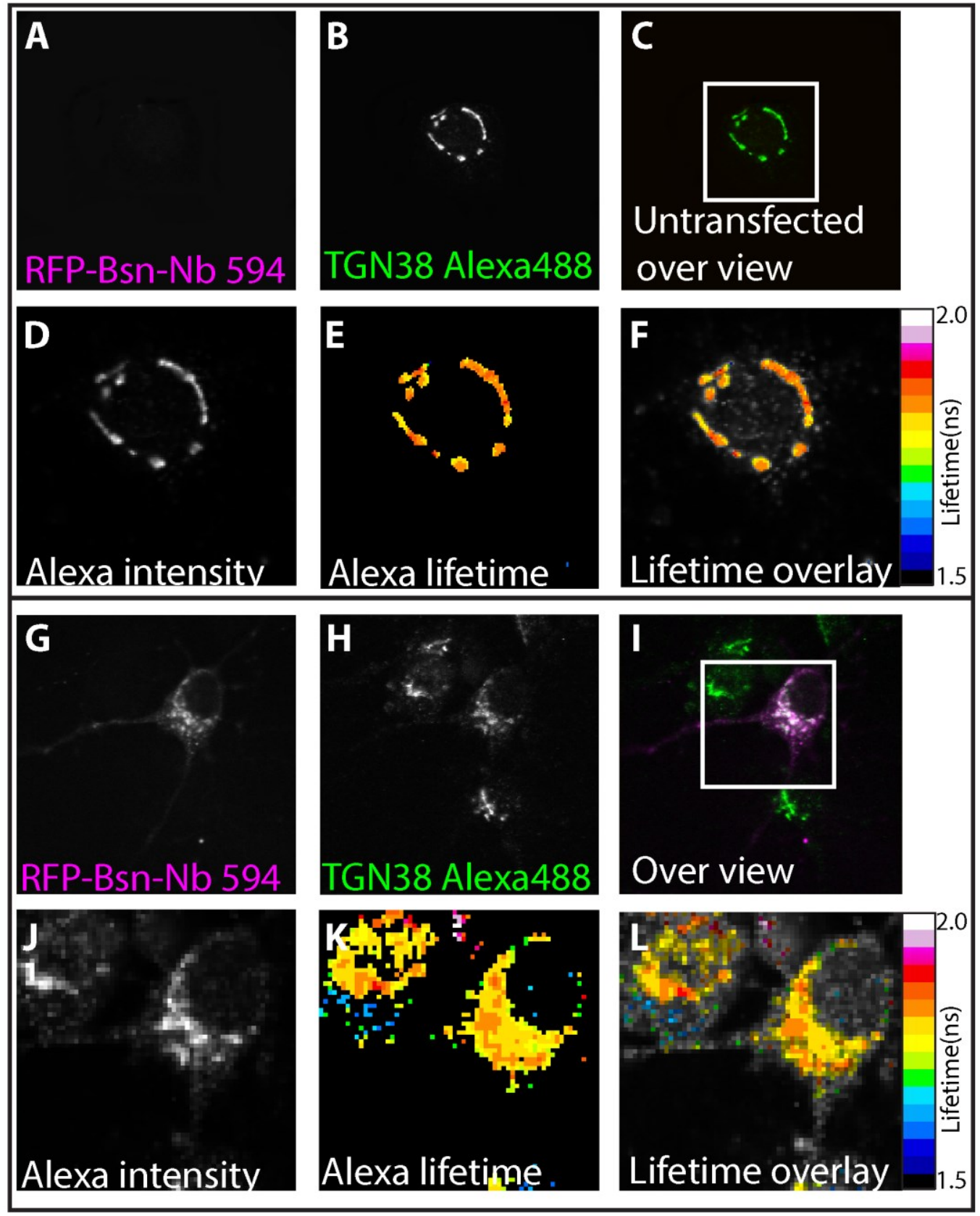




\section{The N-terminus of Bassoon orients the molecule with $6-20 \mathrm{~nm}$ of TGN}

All data so far points at full-length Bassoon molecules being oriented with its $\mathrm{N}$ terminus facing the TGN lamella, where the $97^{\text {th }}$ amino acid RFP tag appears colocalized to the TGN marker signals and is localized within 6 and 20nm from it.

Bassoon molecules appear to consistently be oriented at the TGN, thus the prevalence of random Bassoon orientations can be ruled out, as it would be reflected in the average distributions of its $\mathrm{N}$ - and $\mathrm{C}$-termini values, which would be roughly similar. Centrally symmetric orientations are evident for small synaptic proteins that form oligomers like the postsynaptic protein Homer1, which has been reported to have nearly identical positions for its $\mathrm{N}$ - and $\mathrm{C}$-termini at the post synaptic membrane ${ }^{76}$. Since Bassoon possesses a central oligomerization domain ${ }^{70}$, central symmetry influencing the molecule's orientation is a concern that might be ruled out at the TGN, as the molecule appears extended with significantly different distance distributions of its $\mathrm{N}$ - and $\mathrm{C}$-termini.

In addition, the exact averages distance of $\mathrm{N}$ - and $\mathrm{C}$-terminal Bassoon signals, in the two distance categories, (tabulated in detail in Appendix $\mathrm{G}$ ) shows that $\mathrm{N}$ - and C-termini of Bassoon localize on average in two populations, one that is close and appears localized to the TGN and one population that is further away from the TGN lamella and represents molecules that are being sorted onto transport precursors. On average the closer population of $\mathrm{N}$-terminal signals of Bassoon, irrespective of whether they belong to single- or double-tagged construct, localize $30-32 \mathrm{~nm}$ from the TGN while the C-termini of Bassoon localize on average around $50-60 \mathrm{~nm}$ and indicate that full-length Bassoon molecules extend upto $30 \mathrm{~nm}$ at the TGN (Figure 21). The second population of full-length Bassoon signals have very similar distances of $\mathrm{N}$ - and $\mathrm{C}$-termini of Bassoon molecules, that both localize within 160-220nm from the TGN lamella and reflect the population of equidistantly placed Bassoon molecules on top of transport precursors. To understand the orientation of Bassoon molecule on transport packets an additional study was carried out with full-length single tagged Bassoon constructs on dense-core vesicles, later in this chapter in section 3.6.

Together these results robustly show the orientation of Bassoon at the TGN, where it appears in an extended conformation with its $\mathrm{N}$-terminus facing the TGN lamella and its C-terminus facing away from the lamella (Figure 20). The same orientation of Bassoon was observed in images labeled with TGN38 and Syn6, TGN lamella markers, and from the transfections of single- and double-tagged Bassoon constructs (Figure 17-Figure 19).

This novel finding of an extended orientation of Bassoon at the TGN compartment bears interesting functional implications. It invites the assumption that Bassoon, much like Bassoon and piccolo at synapses, possesses a filamentlike confirmation at the $\mathrm{TGN}^{52,77}$. As Bassoon is localized to the TGN with Piccolo 
and ELKS2 ${ }^{32}$, its extended confirmation may allow the recruitment of others AZPS to form subsets of AZPs that might be loaded onto transport packages together. It might also act a tether between different types of transport vesicles, promoting the larger AZP signal sizes seen at the soma (Figure 5). Additionally the extended confirmation of Bassoon reveals its central oligomerization domain, which in turn could promote clustering and hence recruitment of Bassoon and piccolo molecules to the TGN, so as to properly orient and direct them onto transport precursors.

Comparing the orientation of various deletion constructs of Bassoon at the TGN might cast light onto the implications of Bassoon's extended orientation at both the TGN and the synapse.

Figure 21: A summary of the orientation of full-length Bassoon molecules at the trans-Golgi network.

Transfected DIV7 hippocampal neurons, visualized with two-color STED imaging, immunostained with either trans-Golgi network marker TGN38 or Syn6 and expressing any full-length Bassoon molecule (irrespective of the presence of the myristoyl motif) has high colocalization with the TGN marker at its $\mathrm{N}$-terminus (A) and low colocalization with the TGN marker at its $\mathrm{C}$-terminus (B).A and $\mathbf{B}$ : representative images from Figure 17. C, A diagram demonstrating the orientation and the minimum average distance of the $\mathrm{N}$ - and $\mathrm{C}$-terminal tags of RFP-Bsn-GFP at the TGN lamella. Scale bars $1 \mu \mathrm{m}(\mathbf{A}-\mathbf{B})$.
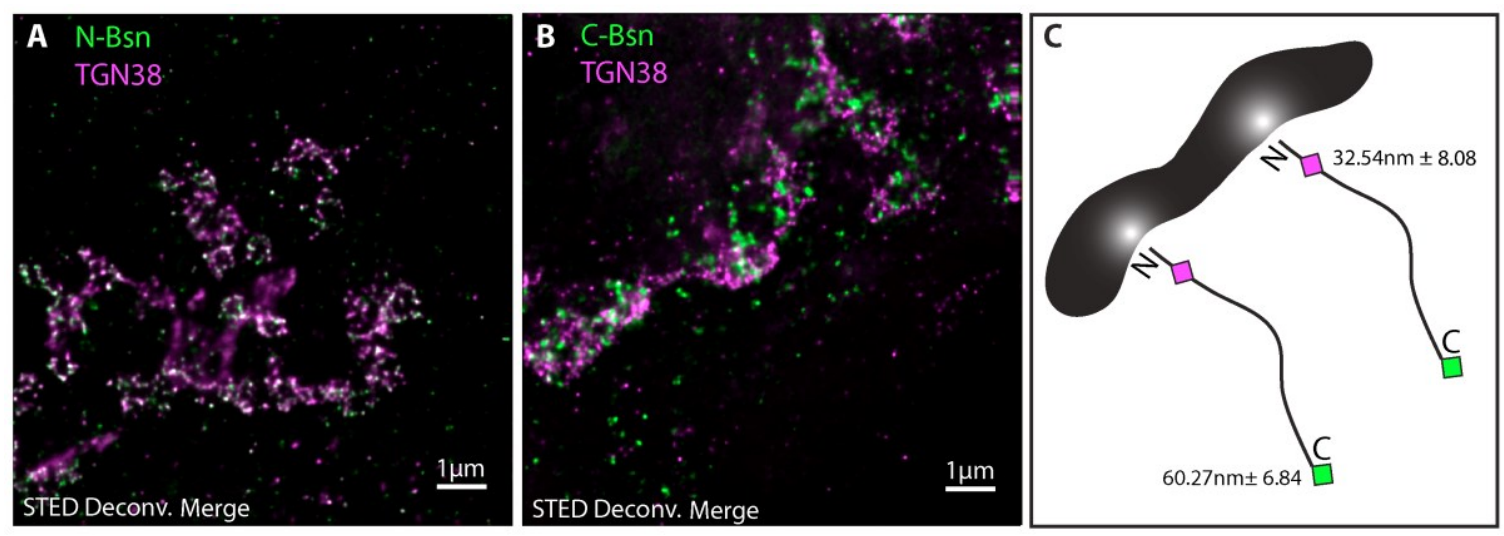


\subsubsection{Localization of Bassoon mutants at the trans-Golgi network}

To understand the influence of Bassoon's orientation at the TGN and to compare the change in orientation brought about by deleted domains and motifs from the Bassoon molecule, first- and second-generation mutant Bassoon constructs are imaged and described in this subsection.

The extensively cited and well-characterized first-generation deletion mutant constructs of Bassoon: GFP-Rbb26Bsn that lacks both the N-and C-terminus of Bassoon molecule (2088-2038a.a.) and GFP-95-Bassoon that lacks the first 94 a.a. of the molecule were visualized with STED microscopy. Using these constructs enabled me to understand the effect the loss of the two termini of Bassoon have on its orientation at the TGN.

In addition, the second-generation myristoyl mutant Bassoon construct was also imaged with STED in order to understand the role the myristoyl group plays in orienting and localizing the $\mathrm{N}$-terminus of Bassoon at the TGN lamella.

\section{Localization of Bassoon constructs lacking one or both termini at the TGN}

The GFP-Rbb26Bsn construct spans from amino acids 2088 to 2563. This sequence contains the coiled-coil 2 (CC2) region of Bassoon and is also known as the Bassoon Golgi Binding Region (BsnGBR), as it is suggested to be the hetero-/homo-oligomerization domain of Bassoon. This construct has been previously shown to accumulate onto Golgi membranes and subsequently decreases the endogenous Bassoon levels at synaptic sites in transfected neurons ${ }^{3}$. In addition, the construct was shown to form intracellular clusters with various sized Bassoon cDNA clones that also contain the CC2 domain, in a yeast two-hybrid assay, promoting its ability to form oligomers ${ }^{30,32}$.

On the other hand, unlike the GFP-Rbb26Bsn construct, the GFP-95Bassoon construct has been shown to successfully traffic and integrate into presynaptic CAZ. Therefore the C-terminus for Bassoon is important for its transport, although the missing $\mathrm{N}$-terminus in this construct may reveal the role these first 94 amino acids have to play in orienting the protein at the TGN.

Both these constructs were transfected in young hippocampal neurons, visualized using the GFP-nanobody-Atto647 at the TGN38-labeled TGN lamella and imaged with two-color STED microscopy following the standard protocol described before. 
As these constructs have been previously characterized in other studies, I have not included their characterization here, although representative confocal images of both constructs at the Golgi can be referred to in Appendix D.

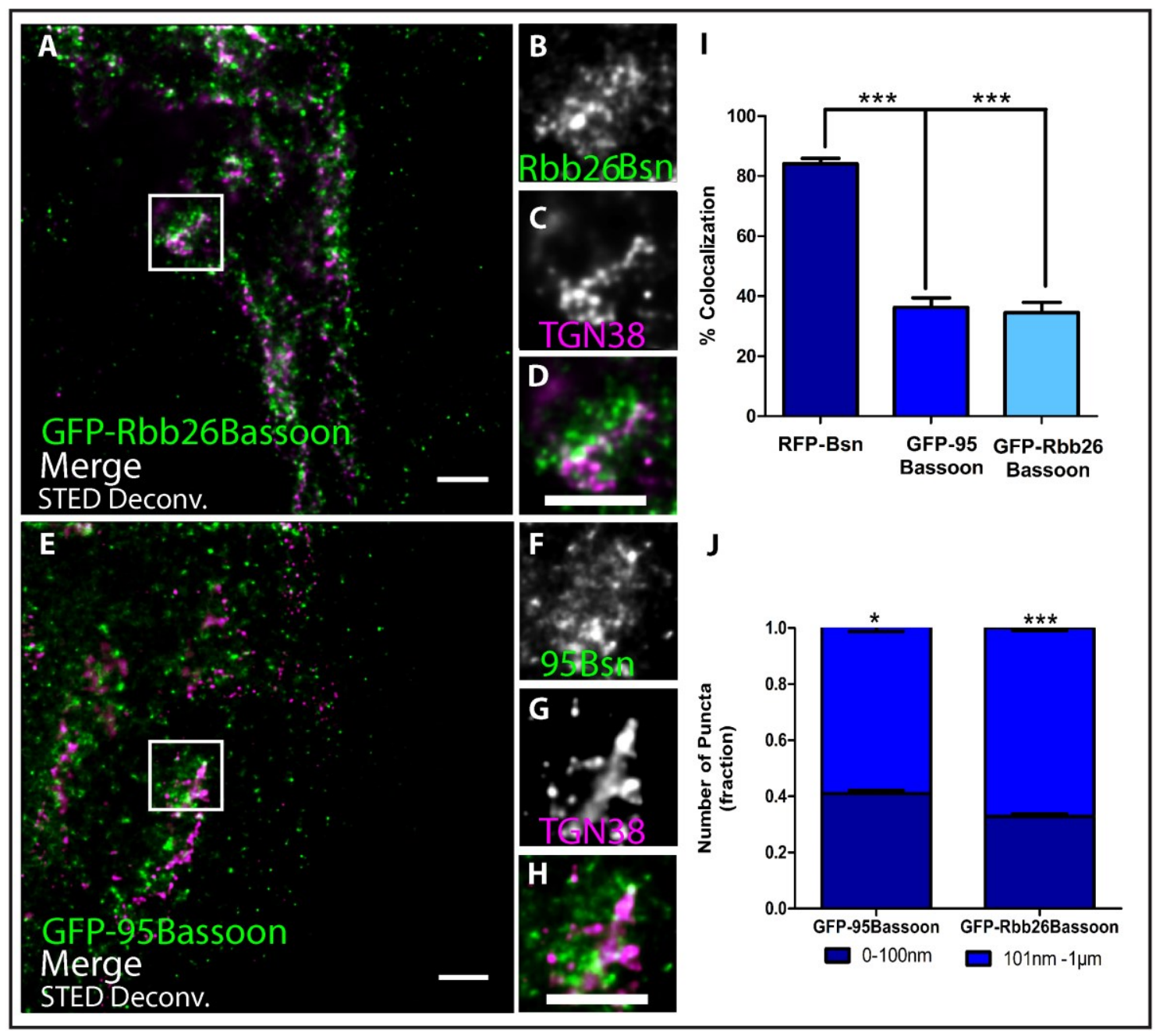

Figure 22: Orientation of first-generation mutant Bassoon constructs at the trans-Golgi network. DIV7 hippocampal neurons transfected with GFP tagged Bassoon mutants devoid of Bassoon's $\mathrm{N}$-terminus, i.e., (95-3938) 95-Bsn construct (A-D), and its $\mathrm{N}$ - and $\mathrm{C}-$ termini (2088-2038), i.e. Rbb26Bsn construct (E-H), were visualized with a GFP-nanobody-Atto647 and TGN38 marker. Insets of the two-color STED deconvolved images in $\mathbf{A}$ and $\mathbf{E}$, are represented in panels $\mathbf{B}-\mathbf{D}$ and $\mathbf{F}-\mathbf{H}$, respectively. Graph I and $\mathbf{J}$ : quantifications the colocalization and signal distributions, respectively. Data are represented as mean $\pm S D, N=10$ cells from two separate experiment, statistically tested with a one-way annova with the Tukey's multiple comparison's post-hoc test * $p$ $<0.05 \&{ }^{* *} p \leq 0.001$. Scale bars $1 \mu \mathrm{m}(\mathbf{A}-\mathbf{P})$.

Both N-terminally tagged deletion mutants 95-Bsn (36.2\%) and Rbb26-Bsn $(41.5 \%)$ constructs have a significantly lower number of colocalizing signals at the TGN38 lamella compared to the RFP-Bsn construct (84.8\%). A small population of colocalized signals also translates into significantly higher populations ( 0.60 population fraction $\pm 0.04 \mathrm{SD}$ ) of the mutant Bassoon signals of 95-Bassoon localized $232 \mathrm{~nm} \pm 41 \mathrm{~nm}$, and Rbb26-Bsn (0.67 population fraction \pm 
$0.03 \mathrm{SD}$ ) are distributed $243 \mathrm{~nm} \pm 29 \mathrm{~nm}$ away from the TGN38 lamella, in the $101 \mathrm{~nm}-1 \mu \mathrm{m}$ distance category (Figure 22 and Appendix $\mathrm{G}$ ).

Despite the low colocalization rate of Rbb26-Bsn at the TGN, it is fascinating that this relatively small piece of Bassoon, spanning 450 amino acids, is still observed in close association to, although not at, the TGN38 signals. This result is similar to the previous observations noted for the C-terminal signals of full-length Bassoon and implies that like full-length Bassoon, Rbb26-Bsn is correctly recruited to the TGN but maybe is incorrectly organized at the TGN. These observations could be a caused by the CC2 domain of Bassoon, expressed by the Rbb26-Bsn construct, oligomerizing with endogenous Bassoon molecules, which are correctly oriented at the TGN.

In comparison, the 95-Bassoon signals also were observed associated to the TGN but not colocalizing with it and presented a signal distribution pattern similar to the C-termini of full-length Bassoon. As the 95-Bassoon construct also contains a functional CC2 domain, its association to the TGN may also be a result of the molecule being correctly recruited, but incorrectly organized, at the TGN by the Rbb26 domain.

Nonetheless, both constructs lack a significant portion of Bassoon's N-terminus, which in turn distinctly reduces the colocalization of the molecules at the TGN lamella. This result reveals that the lack of the N-terminus of Bassoon deprives the molecules from being correctly localized at the TGN and shows that the first 94 amino acids of Bassoon are important for Bassoon to attain its extended orientation at the TGN.

Unpublished EM data from our lab show DAB precipitates of 95-Bassoon loaded onto a large number of clear-core vesicles and a handful of dense-core vesicles in the soma (Dresbach and Wittenmayer, unpublished). This observation deviates from the relatively small population of endogenous AZP signal sizes corresponding to clear-core vesicles observed in Figure 5 and suggests that 95Bassoon may be incorrectly loaded on the wrong population of transport carriers.

In light of the role of the $\mathrm{N}$-terminal 94 amino acids in orienting the Bassoon molecule to the TGN lamella, the EM observation from Dresbach and Wittenmayer (unpublished), provides a vital link between the orientation of the molecule and its correct sorting and loading onto transport vesicles. Though to understand how the orientation of Bassoon facilitates these processes, a detailed understanding of how the N-terminus of Bassoon and TGN lamella interact is needed. 


\section{Role of the myristoyl group of Bassoon in orienting the molecule at the TGN}

An ideal motif within Bassoon's $N$ terminus, to study this question, is its hydrophobic myristoyl motif at the very $\mathrm{N}$-terminal tip of the molecule. The second-generation constructs were designed with the purposeful intention to promote the myristoyl group's inherent interactions during orientation, transport and assembly of AZPs. To reveal these interactions a point mutated Bassoon construct was created, in which glycine was changed to an alanine, at the $2^{\text {nd }}$ amino acid position therein destroying the myristoyl motif. This construct characteristically accumulates around the TGN in young neurons and gets assimilated at synaptic sites (Figure 13). Although this construct does not appear to affect the transport of the recombinant protein, it may be the interaction site between Bassoon's $\mathrm{N}$-terminus and the TGN lamella as myristoyl groups are known to preferentially integrate into the lipid layers of membranes ${ }^{103}$.

To dissect in detail the exact means by which the $\mathrm{N}$-terminus of Bassoon promotes its extended orientation at the TGN, the myristoyl mutant construct, G2A-RFP-Bsn-GFP, is imaged in comparison to the myristoyl containing RFPBsn-GFP construct.

As with the observations of the previous subsection the G2A myristoyl mutant also possess a function $\mathrm{CC} 2$ oligomerization domain that may, via oligomerization events with endogenous Bassoon, influence its localization to the TGN. In order to visualize the true potential of the myristoyl group in orienting Bassoon at the TGN, the myristoyl mutant is visualized with STED in the normal rat cultures, as well as a in $B s n^{-/}$knockout mice cultures that provide an endogenous Bassoon free environment.

\section{Orientation of the myristoyl Bassoon mutant at the TGN}

DIV7 rat hippocampal neurons transfected with the myristoyl mutant G2A-RFPBsn-GFP were imaged with STED microscopy using the standard protocol previous described. The $\mathrm{N}$ - or $\mathrm{C}$-termini of the mutant Bassoon molecules were labeled with either the RFP/GFP nanobody at the TGN38 marker. Images of fulllength RFP-Bsn-GFP that were labeled and imaged identically were compared to G2A-RFP-Bsn-GFP images to gauge their difference in orientation. 
Figure 23: Comparing the orientation of the double-tagged myristoyl mutant Bassoon construct to the double-tagged full-length the trans-Golgi network.

DIV7 hippocampal neurons transfected with mRFP-Bsn-mEGFP $(\mathbf{A}-\mathbf{H})$ and myistoyl mutant G2A-mRFP-Bsn-mEGFP (I-P). These two-color STED images were compared by visualizing their $\mathbf{N}-(\mathbf{A}-\mathbf{D}$ and $\mathbf{I}-\mathbf{L})$ and $\mathbf{C}-(\mathbf{E}-\mathbf{H}$ and $\mathbf{M}-\mathbf{P})$ termini, respectively using RFP-nanobodyAtto594 or GFP-nanobody-Atto647 and TGN38 marker. Graph $\mathbf{Q}$ and $\mathbf{R}$ were represented as mean $\pm \mathrm{SD}, \mathrm{N}=10$ cells from two separate experiment for quantifying the colocalization and signal distributions, respectively, ${ }^{* \star *} p \leq 0.001$. Scale bars $1 \mu \mathrm{m}(\mathbf{A}-\mathbf{P})$.

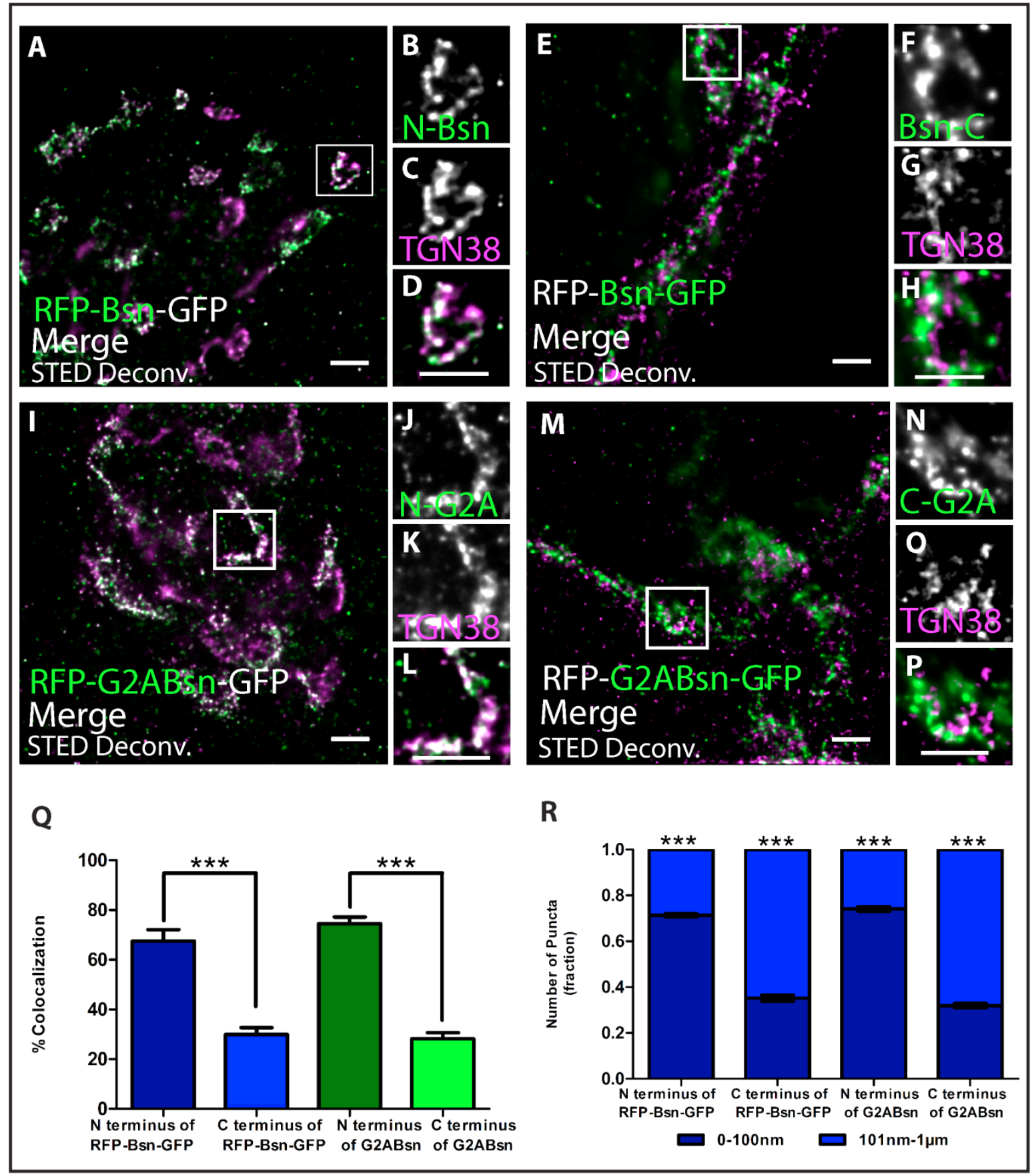


Surprisingly, the $\mathrm{N}$ - and C-termini of the G2A-Bsn mutant had similar colocalization and signal distribution as the non-mutant full-length Bassoon termini.

The N-termini of the double tagged G2A-Bsn and full-length Bassoon had high percentages of colocalizing signals, of $74.5 \%$ and $67.5 \%$ respectively, at the TGN38 signals. In comparison their respective C-termini, showed significantly lower colocalizations of $28.2 \%$ (G2A-Bsn) and 30\% (RFP-Bsn-GFP) at the TGN38 signals. Similarly, the signal distribution trends favored a significantly larger fraction of the N-terminal signals for G2A-Bsn (0.75 \pm 0.02 SD) and RFPBsn-GFP $(0.71 \pm 0.02 \mathrm{SD})$ localized within the $0-100 \mathrm{~nm}$ category, while the Cterminal signals had significantly larger fractions of their signals distributed $101 \mathrm{~nm}-1 \mu \mathrm{m}$ from the TGN38 signals for G2A-Bsn (0.68 $\pm 0.02 \mathrm{SD})$ and RFPBsn-GFP (0.65 \pm 0.04 SD) (Figure 23).

This result reveals that the myristoyl group does not significant influence the orientation of the Bassoon molecule at the TGN as the signal distributions of the $\mathrm{N}$ - and $\mathrm{C}$-termini of this mutant Bassoon molecule are similar to the non-mutated tagged Bassoon molecules. These results also suggest that the orientation of Bassoon might be heavily influenced by the presence of properly oriented endogenous Bassoon molecules that might organize recombinant Bassoon molecules at the TGN if they possess a functional CC2 domain. Additionally this experiment does not reveal if the myristoyl group is the interaction site between Bassoon's N-terminus and the TGN lamella.

\section{Orientation of the myristoyl Bassoon mutant in Bsn ${ }^{-/}$knockout and $\mathrm{Bsn}^{+/+}$ littermate wildtype mice}

To reveal the role of Bassoon's myristoyl group at the TGN the G2A-RFP-BsnGFP was transfected in $B s n^{-/}$knockout mice neurons, which were devoid of any Bassoon except the administered mutant G2A-Bsn molecules. As the orientation of Bassoon had thus far been studied only in rat hippocampal neurons the orientation of G2A-Bsn molecules were additionally recorded from G2A-RFPBsn-GFP transfected $\mathrm{Bsn}^{+/+}$wildtype littermate mice neurons and compared to $B s n^{-/}$knockout mice neurons. The labeling and imaging protocol for acquiring the two-color STED images were identical with the exception of use of a TGN38 antibody raised in rabbit instead of the traditionally used mouse antibody. 

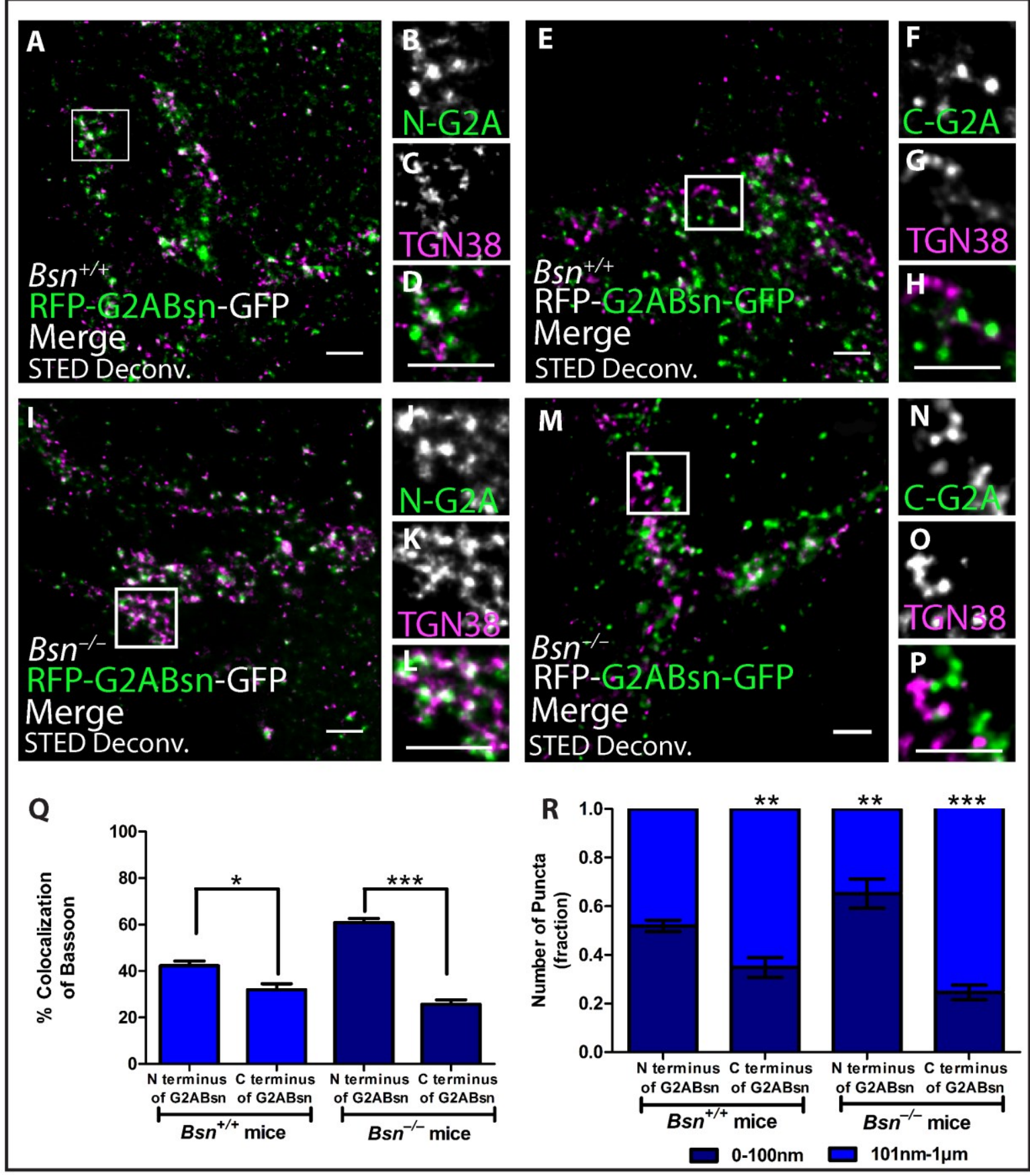

Figure 24: Orientation of G2A-mRFP-Bsn-mEGFP myristoyl mutant construct in endogenous Bassoon-free $\mathrm{Bsn}^{-/}$knockout mice and their $\mathrm{Bsn}^{+/+}$wildtype littermates.

DIV7 $\mathrm{Bsn}^{+/+}(\mathbf{A}-\mathbf{H})$ and $\mathrm{Bsn}^{-/}(\mathbf{I}-\mathbf{M})$ sandwich hippocampal cultures were transfected with G2AmRFP-Bsn-mEGFP. Two-color STED images with their respective insets are shown for the N(A-D and $\mathbf{I}-\mathbf{L})$ and $\mathbf{C}-(\mathbf{E}-\mathbf{H}$ and $\mathbf{M}-\mathbf{P})$ termini of the myristoyl mutant construct, respectively. Immunostaining was performed using RFP-nanobody-Atto594 or GFP-nanobody-Atto647 and TGN38 marker. Graph $\mathbf{Q}$ and $\mathbf{R}$ were represented as mean $\pm \mathrm{SD}, \mathrm{N}=8$ cells from two knockout and two wildtype animals and quantified for amount of colocalization and signal distributions, respectively, ${ }^{*} p \leq 0.05,{ }^{* *} p \leq 0.01$ and ${ }^{* *} p \leq 0.001$. Scale bars $1 \mu \mathrm{m}(\mathbf{A}-\mathbf{P})$. 
The $\mathrm{N}$ - and C-termini of the G2A-Bsn mutant in WT and $\mathrm{KO}$ mice had similar colocalization and signal distribution trends as were observed in rat G2A-Bsn neurons.

The N-termini of the G2A-Bsn in mice neurons had higher percentages of colocalizing signals at $42.3 \%$ for WT neurons and $60.9 \%$ for $\mathrm{KO}$ neurons in comparison to their respective C-termini, which were only colocalized by $31.9 \%$ (WT) and $25.7 \%(\mathrm{KO})$ at the TGN38 signals. Subsequently, the signal distributions also trended towards the larger fraction of the $\mathrm{N}$-terminal signals in G2A-Bsn transfected WT $(0.52 \pm 0.04 \mathrm{SD})$ and KO $(0.65 \pm 0.10 \mathrm{SD})$ neurons localized within the $0-100 \mathrm{~nm}$ distance category, and the larger fraction of $\mathrm{C}$ terminal G2A-Bsn signals in WT $(0.65 \pm 0.07$ SD) and KO (0.75 \pm 0.05 SD) neurons were distributed in the $101 \mathrm{~nm}-1 \mu \mathrm{m}$ category (Figure 24).

This observation reveals that full-length Bassoon molecules are not localized close to the TGN by simply oligomerizing with endogenous Bassoon molecules. This is evident because Bassoon's extended orientation is visible not only in rat neurons, but also in WT and endogenous Bassoon-free KO mice neurons. It also re-emphasizes the importance of Bassoon's N-terminus in orienting and promoting colocalization of Bassoon molecules at the TGN lamella. It becomes evident now that the myristoyl motif in Bassoon's N-terminus is not the site responsible for the orientation or the interaction of the $\mathrm{N}$-terminus of Bassoon molecules at the TGN. The persistence of high colocalizations of Bassoon signals, at the TGN, in the absence of both myristoyl motif and endogenous Bassoon suggests that there might be other interaction sites and sequences within the first 94 amino acids that orient the $\mathrm{N}$-termini of Bassoon molecules within 6 and $20 \mathrm{~nm}$ of the TGN38 signals.

\subsection{Orientation of Bassoon during PTV transport}

To understand whether the AZP orientation on transport precursors plays a role in priming the assembly of the protein at the CAZ, full-length N-terminally tagged mRFP-Bsn or C-terminally tagged Bsn-mRFP were visualized on GFPChromograninA (CGA) carrying dense-core vesicles. As Bassoon has been shown with super resolution microscopy to be loaded onto CGA positive densecore vesicles in the axons of developing neurons ${ }^{71}$ and is the only AZP protein for which $\mathrm{N}$ - and C-terminally constructs are currently available, it was naturally the best choice to visualize its orientation on trafficking dense-core vesicles. Twocolor STED imaging was performed for both Bassoon termini, labeled with the RFP nanobody, on CGA-positive dense-core vesicles, labeled with the GFP nanobody. 


\subsubsection{Orientation of the $\mathrm{N}$ - and $\mathrm{C}$ - termini of Bassoon around chromogranin A positive transport packets}

Both $\mathrm{N}$ - and $\mathrm{C}$ - terminally tagged Bassoon and CGA positive puncta are seen in high intensities at juxtanuclear positions in young DIV6 transfected neurons. There appears to be low colocalizations of both AZP and tagged vesicle cargo at these juxtanuclear locations. This signifies that the AZP are either not yet loaded on to CGA positive vesicles, and/or are localized to different Golgi planes, or are associated at least $20 \mathrm{~nm}$ from the core of the vesicles. Close inspection of the punctate signals of $\mathrm{N}$-terminally and $\mathrm{C}$-terminally tagged Bassoon molecules in the axon reveals with great clarity that CGA positive dense-core vesicles carry Bassoon on them, as is indicated by the Bassoon signal surrounding the CGA signal. The axonal confocal punctate signals after being resolved with STED reveal from a couple to a few CGA positive vesicles trafficking Bassoon within them.

In many instances these resolved puncta contain Bassoon signals that look like they are being dragged along with the CGA vesicles. Interestingly though, neither the $\mathrm{N}$ - nor the C-terminus of Bassoon is arranged differently around CGA signals and neither termini are visually colocalized to the CGA positive vesicle signals (Figure 25).

This result suggests that Bassoon does not possess a stretched open or linear confirmation during its transport on CGA positive dense-core vesicles. This observation reflects that large AZP proteins like Bassoon may be favorably folded during their transport to synaptic sites, thereby reducing the likelihood that they are oriented to promote preassembly AZPs during transport.

Figure 25: Orientation of full-length single-tagged Bassoon constructs to chromogranin A (CGA) positive PTVs in the soma and down the axon.

DIV7 hippocampal neurons double transfected with mRFP-Bsn or Bsn-mRFP and GFP-CGA and immunostained with the RFP-nanobody-Atto 594 and GFP-nanobody-Atto 647. A-C and H-J, represent confocal over views and two-color STED images of $\mathrm{N}$ - and C-terminally tagged Bassoon around CGA packets in the soma. $\mathbf{D}$ and $\mathbf{K}$ represent confocal axonal over views of the same transfected neurons shown in $\mathbf{A}-\mathbf{C}$ and $\mathbf{H}-\mathbf{J}$, respectively. Insets $\mathbf{E}, \mathbf{F}, \mathbf{M}$, and $\mathbf{N}$ represent two-color deconvolved STED images of central axonal regions. Zooms of trafficking packets labeled for the $\mathrm{N}$-terminus $(\mathbf{G}-\mathbf{H})$ and the $\mathrm{C}$-terminus $(\mathbf{M}-\mathbf{N})$ of Bassoon. $\mathrm{N}=8$ for two images per area per neuron from one set. Scale bars confocal over views $5 \mu \mathrm{m}(\mathbf{A}, \mathbf{H}), 10 \mu \mathrm{m}(\mathbf{C}, \mathbf{J})$, STED images $1 \mu \mathrm{m}(\mathbf{B}-\mathbf{F}, \mathbf{J}-\mathbf{L})$ and $0.5 \mu \mathrm{m}(\mathbf{E}, \mathbf{F}, \mathbf{M}$, and $\mathbf{N})$. 


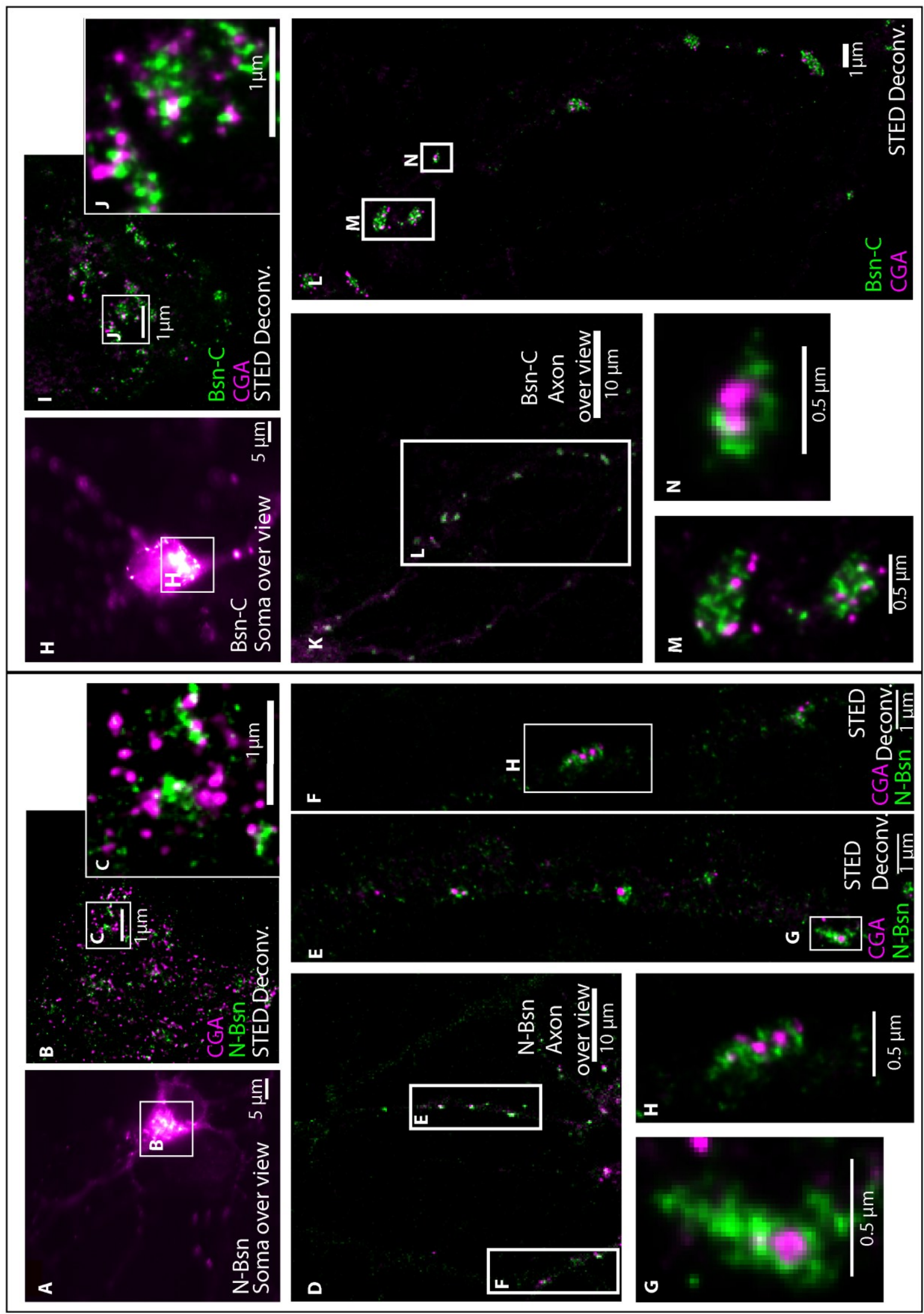




\subsection{Organization of Bassoon molecules at the Golgi and synaptic compartments}

The extended orientation of the Bassoon molecules at the TGN in immature neurons resembles observations of Bassoon's orientation in mature presynaptic boutons. Bassoon's orientation at synapses has been implicated in providing a filament-like backbone for other CAZ proteins to bind to and guide synaptic vesicles from the their pool to the fusion site ${ }^{76,77}$. Similarly, Bassoon's extended orientation at the TGN may also promote interactions and local mechanisms. To understand these, a detailed examination of the characteristics conferred by the extended orientation needs to be studied.

Inspiration was drawn from the observation where small fragments of Rbb26-Bsn molecules were organized at the TGN, despite their lack of an $\mathrm{N}$ - or C-terminus (Figure 22). This observation highlighted the fact, that like orientation, the organization of a molecule at a subcellular compartment might influence its correct processing at specific substructures.

To visualize the organization of Bassoon molecules at the TGN, single-tagged full-length Bassoon constructs, characterized in Figures 4 and 5, were cotransfected in pairs of either $\mathrm{N}$ - or C-terminally tagged constructs. FLIM imaging of the transfected neurons was performed to assess the proximity the $\mathrm{N}$ - or $\mathrm{C}$ termini of neighboring molecules had to each other.

In addition, a similar experiment to visualize Bassoon's organization in synaptic sites, which hasn't yet been studied, was also performed to estimate whether the extended orientation of Bassoon, at the TGN and at synapses, encouraged similar organizations of the Bassoon molecules. 


\subsubsection{Organization of Bassoon molecules in the soma}

DIV10 hippocampal neurons were co-transfected with either the N-terminally tagged mRFP-Bsn and mEGFP-Bsn or the C-terminally tagged Bsn-mRFP and Bsn-mEGFP constructs, fixed at DIV14 and imaged for their difference in fluorescence lifetimes compared to the mEGFP-Bsn control. The GFP and RFP tags on Bassoon molecules were boosted with the application of a polyclonalGFP polyclonal antibody labeled with Alexa488 and the RFP-nanobody-Atto594. This would generated a $\sim 30 \mathrm{~nm}$ cloud around each GFP tag and $\sim 13 \mathrm{~nm}$ cloud around the RFP tag, in an effort to bring these signals closer for quantifiable FRET to occur. FLIM imaging was performed in a $5 \mathrm{x}$ zoom of the over view image, clearly representing the neuronal soma, which was centered at a $z$ position that presented with the highest intensity of Bassoon signals at juxtanuclear positions. The GFP tags of Bassoon boosted with Alexa488 were recorded for their lifetimes and the FRET efficiencies for the control and the cotransfected $\mathrm{N}$ - and C-terminal Bassoon images were calculated, normalized, fitted to a Gaussian distribution and plotted.

FRET efficiencies were centered on $0 \%$ and were represented by a blue to green LUT color range for the control mEGFP-Bsn molecules in the soma, since these molecules have no acceptor molecules to FRET with. No FRET was measured between the C-termini of GFP and RFP Bassoon constructs as seen in Figure 24 $\mathrm{H}-\mathrm{K}$, represented by the cooler bluish-green LUT color, as their $0.085 \%$ FRET efficiency was insignificantly different from the control. The N-termini of Bassoon on the other hand showed significant higher FRET efficiencies of $3.73 \%$ represented by a warmer yellowish LUT color representation, indicative of positive FRET (Figure 26).

These results are indicative of significantly closer proximities between the $\mathrm{N}$ termini of neighboring Bassoon molecules oriented at the TGN, while the boosted C-termini signals appear to be localized at least $5 \mathrm{~nm}$ away from each other.

The close organization of the $\mathrm{N}$-termini of full-length Bassoon molecules emphasizes the molecule's orientation at the TGN lamella. This result provides a link between the orientation and organization of AZPs at the TGN and also highlights the importance of Bassoon's N-terminus, in not only orienting but organizing the open conformation Bassoon molecules such that they may be open to interactions with other AZPs for co-transport or adapter protein on transport carriers. 

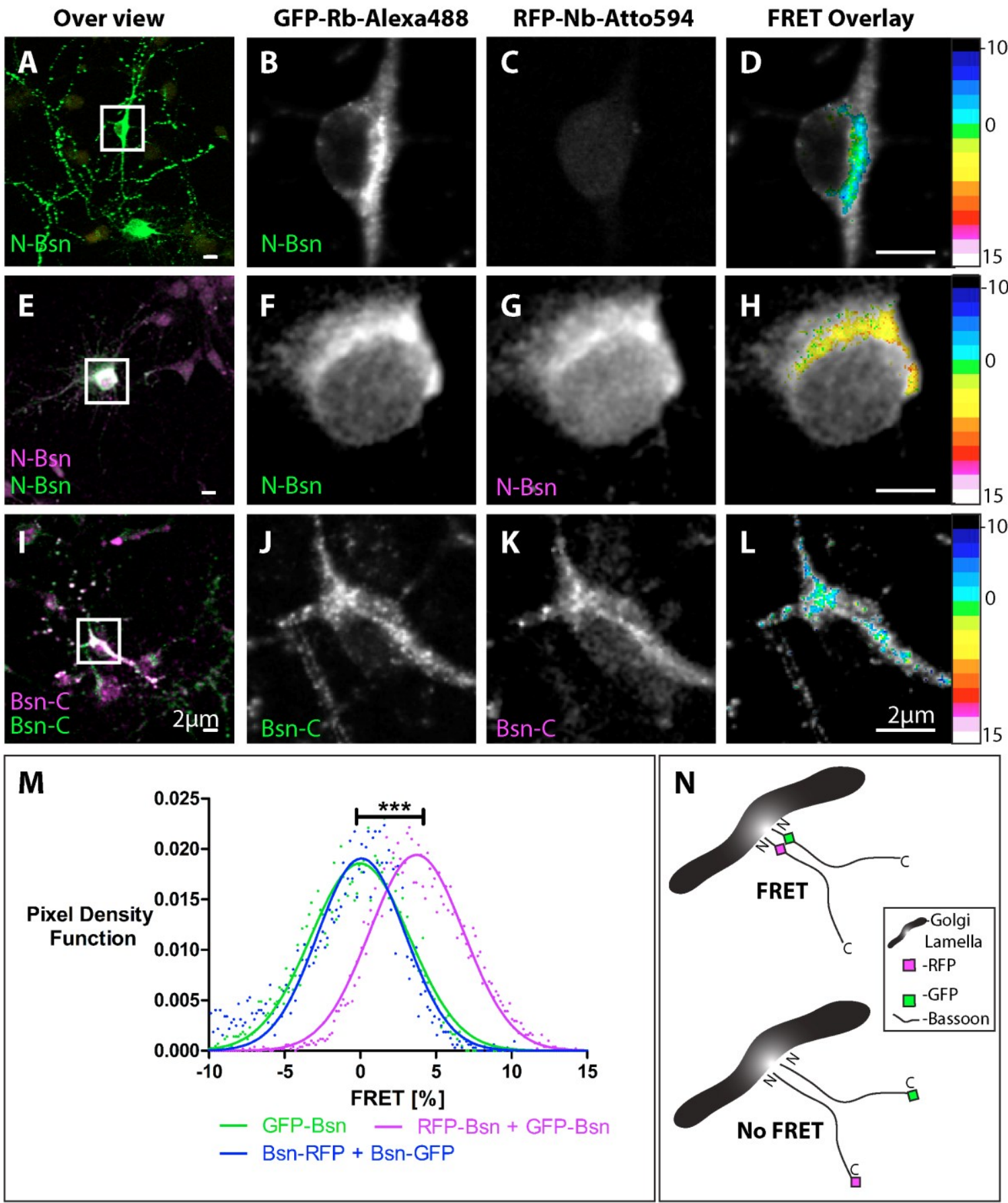

Figure 26: Organization of neighboring Bassoon molecules at the soma. Single-tagged full-length Bassoon constructs, double transfected in pairs of mRFP-Bsn and mEGFP-Bsn (E-H) and BsnmRFP and Bsn-mEGFP (I-L) and compared to mEGFP-Bsn control A-D were recorded for their GFP-Alexa 488 lifetimes in DIV7 hippocampal neurons, labeled with the GFP-Alexa 488 and RFP-nanobody-Atto594. A, E and I are immunofluorescence over view images of the transfection and their insets are reflected in panels $\mathbf{B}-\mathbf{D}, \mathbf{F}-\mathbf{H}$, and $\mathbf{J}-\mathbf{L}$. Graph $\mathbf{M}$ represents the normalized and Gaussian fitted lifetime pixel density over a 10-minute recording and plotted for their FRET efficiency. $\mathrm{N}=6$, Unpaired Student's $t$ test, ${ }^{* * *} p \leq 0.001$. $\mathrm{N}$ : a diagram to represent the organization of the termini of the Bassoon molecules at the TGN. Scale bars $2 \mu \mathrm{m}(\mathbf{A}-\mathbf{K})$.

16-color LUT reflects FRET efficiencies from $-10 \%-+15 \%$ FRET. 


\subsubsection{Organization of Bassoon molecules at the synapse}

It has been shown that Bassoon is oriented with its C-terminus facing the presynaptic membrane and localized $30 \mathrm{~nm}$ from it, while its $\mathrm{N}$-terminus faces into the presynaptic bouton and is localized $80 \mathrm{~nm}$ from the plasma membrane ${ }^{76,77}$.

As Bassoon molecules are extended both at the TGN and at the synapse they might share similar characteristics that are conferred by their orientation. To determine whether the extended orientation of Bassoon promotes the organization of the molecules or not, a similar FLIM experiment was performed in synaptic sites of DIV14 neurons.

Co-expressed C-terminally or N-terminally tagged EGFP and RFP fusion constructs of Bassoon, as well as the mEGFP-Bsn control neurons were imaged without boosting the GFP and RFP tags. Since a synaptic site is roughly five times smaller ${ }^{109}$ than a neuronal soma and contains fewer Bassoon molecules, the distance between neighboring molecules should be small enough to visualize direct FRET between GFP and RFP molecules. Over view images of potential synaptic sites from transfected DIV14 neurons were located by tracking a neuronal process far away from the soma of the neuron, to sites that have transfected Bassoon puncta in close proximity to projections or somas of untransfected neurons. Such sites are favorable for creating synapses and an average of $63 \%$ of second-generation full-length Bassoon signals have been shown to accumulate at synapses (Figure 12). Zooms of these over view images were then focused to have high intensities for all synaptic spots in the image and the GFP fluorescence lifetimes of these images were recorded. FRET efficiencies were calculated from normalized lifetime distributions of the raw images, which were fitted to a Gaussian distribution and plotted.

Quite remarkably, a similar organization pattern, as was observed at the TGN, for the $\mathrm{N}$-terminal, C-terminal and the control Bassoon molecules at the synaptic sites as. The FRET efficiencies of the control mEGFP-Bsn (0\%) and the cotransfected C-terminal constructs: Bsn-mEGFP and Bsn-mRFP (1.62\%) were marginally different, represented by bluish to green LUT range at synaptic sites, while the N-termini of Bassoon showed FRET efficiency of $7.26 \%$, which were represented by a yellowish-red LUT color, indicative of positive FRET (Figure 27).

This result shows that the organization of Bassoon molecules at the synapse promotes localization of the $\mathrm{N}$-termini of Bassoon within $5 \mathrm{nms}$ and implies that they bundle together in the CAZ scaffold, which might be responsible for the triangular dense structures of $A Z s$ often reported at the presynaptic membrane in classical EM images. It also shows that the C-termini are farther than $5 \mathrm{~nm}$ apart and reflects that the CAZ structure has a larger base and might be responsible for separating two vesicle-docking stations at the presynaptic plasma membrane. 
Figure 27: Organization of Bassoon molecules at synaptic sites.

Single-tagged full-length Bassoon constructs, double transfected in pairs of mRFP-Bsn and mEGFP-Bsn (E-H) and Bsn-mRFP and Bsn-mEGFP (I-L) and compared to mEGFP-Bsn control $\mathbf{A}-\mathbf{D}$ were recorded for their GFP lifetimes in DIV7 hippocampal neurons. A, E and I are immunofluorescence over view images of the transfection and their insets are reflected in panels B-D, F-H, and J-L. Graph N represents the normalized and Gaussian fitted lifetime pixel density over a 10-minute recording and plotted for their FRET efficiency. $\mathrm{N}=6$, Unpaired Student's $t$ test, ${ }^{* *} p \leq 0.001$. N: a diagram to represent the organization of the termini of the Bassoon molecules at the synaptic sites. Scale bars $2 \mu \mathrm{m}(\mathbf{A}-\mathbf{K})$. 16-color LUT reflects FRET efficiencies from $-10 \%-+15 \%$ FRET.
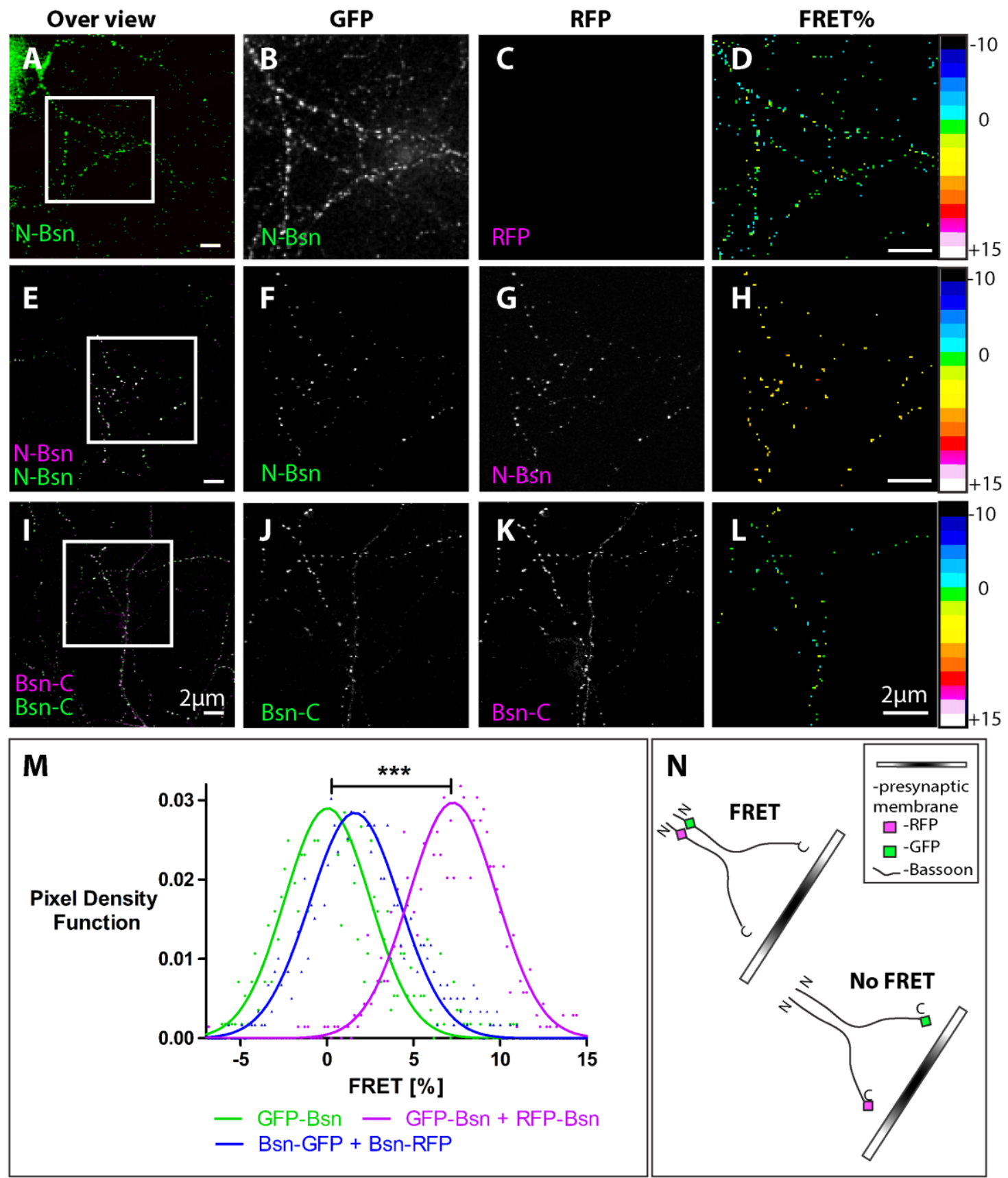


\section{Chapter 4}

\section{Discussion}

The CAZ is presynaptic scaffold of proteins that regulates synapse assembly and function ${ }^{110}$. The sequence of events that cause these proteins to assemble, exactly opposite the post-synaptic scaffold, in mammalian synapses is still poorly understood. Despite two decades of research addressing the function and roles of each individual AZP in the CAZ, the mechanisms that mediate the assembly of the CAZ structure are yet to be understood ${ }^{9,110}$. Nanoscopic observations of these proteins have started to bring in new evidence that could help unravel assembly mechanisms ${ }^{76,77}$ by linking the structure and localization of these proteins to their function in the CAZ complex.

In this study, I have used super-resolution microscopy to reveal that different subsets of AZPs are distributed to specific Golgi subcompartments. This is their first site of localization in neurons and the sorting site for AZPs to be loaded onto a range of different transport precursors. AZP signals were predominantly observed on sizes that corresponded to the presence of small clusters of clearand dense-core transport vesicles that may bring different AZPs in close proximity to each other. This trend of AZP distribution suggests the presence of early preassembly and sorting mechanisms for AZPs in the soma, and highlights the Golgi as the first modulatory station in their journey to the presynaptic membrane.

Trafficking AZPs Bassoon, Munc13-1 and Piccolo, were uniformly distributed in the neuronal axon and STED imaging revealed a much smaller population, than 
previously reported, of Bassoon colocalizing, and hence co-trafficking, with Munc13-1 and Piccolo in axons. This indicates that the sorting mechanism in the soma may have a larger effect on the composition of AZPs transported to nascent synapses.

In addition, Bassoon, one of the two largest AZP molecules, was discovered to possess an extended orientation at its Golgi compartment. The N-terminus of fulllength Bassoon was seen colocalized within 6-20nm from a TGN marker, while the C-terminus did not colocalize and faced away from the TGN. The N-terminus of Bassoon is therefore implicated in localizing and orienting the molecule at the TGN. The myristoyl motif within Bassoon's N-terminus does not influence its orientation at the TGN, although deletion mutant proteins lacking either both termini of Bassoon (Rbb26-Bsn) or the N-terminus of Bassoon (95-Bsn) lose their colocalization to the TGN lamella. This makes the first 94 amino acids of Bassoon's N-terminus essential for its proper localization and orientation at the TGN and highlights the ability of the central CC2 domain of Bassoon (expressed by the Rbb26-Bsn construct) to sufficiently recruit Bassoon molecules to the Golgi.

Bassoon molecules possess an extended conformation at the TGN (Figure 17Figure 19) and at mature synapses ${ }^{76,77}$; this conformation uniquely presents itself with the characteristic property of neighboring Bassoon molecules organized with their $\mathrm{N}$-termini in close proximity to each other and their C-termini localized at least $6 \mathrm{~nm}$ apart at both subcellular localizations. Interestingly, the $\mathrm{N}$ - and $\mathrm{C}$ termini of Bassoon molecules on CGA positive trafficking DCVs were positioned on top of the DCVs, equidistant from its core, suggesting that Bassoon no longer possess an extended conformation on transporting vesicles, in order to facilitate its transport as a large, albeit, more compact cargo. This change in conformation of Bassoon from extended at the TGN, to compact on trafficking vesicles and back to being extended at synaptic sites is the first hint towards a mechanism that influences the sequential assembly of the CAZ complex. The orientation and organization of the Bassoon molecule, at different subcellular destinations, bears interesting functional implications on local mechanisms at these structures and helps to allude towards additional underlying mechanisms that govern the proper assembly of the CAZ structure.

\subsection{Localization at the Golgi}

\subsubsection{Active zone proteins selectively localize to different Golgi subcompartments}

AZPs are first observed in young neurons localized to the Golgi and selectively get recruited to specific Golgi subcompartments. Bassoon, Piccolo and ELKS2 have been shown to specifically localize to the trans-Golgi Network, Munc13-1 is 
observed at the cis-Golgi lamella, and RIM1 $\alpha$ is diffused in the soma and in young neurites (Figure 3, Figure 4 and ref. $^{32}$ ). While Rim $1 \alpha$ 's localization is only observed later in development, on RIM-Neurexin-CASK-voltage-gated $\mathrm{Ca}^{2}$ channels (VGCCs) transport units in the axon ${ }^{3}$, the other CAZ proteins undergo sorting and assembly onto transport carriers at their respective Golgi compartments. Using STED microscopy, I observed endogenous AZPs at their designated Golgi compartment as punctate signals decorating regions at and around the Golgi lamella, and, unlike previous observations with confocal microscopy, these AZP localizations were not uniformly spread all over the Golgi signals. This localization of AZPs suggests the presence of specialized sites at their Golgi subcompartment where the proteins are sorted and loaded onto unique transport carriers. The properties of the transport carriers, conferred by the Golgi compartment they are generated from, will ultimately define the subsets of AZPs that co-traffic out together from the soma and the route of transport they take down the axon based on the microtubule adaptor proteins they possess (Figure 28).

Similar localizations of invertebrate AZ proteins Liprin and Syd2 ${ }^{111}$ and presynaptic proteins such as synaptic vesicle marker 2 (SV2) ${ }^{112}$, synaptophysin ${ }^{113}$, SNAP25, and VAMP2 ${ }^{114}$ have also been reported at the TGN network with light and confocal microscopy. In addition, TGN-derived vesicles have been shown to supply developing inhibitory synapses by shuttling $G_{A B A}$ receptors, their associated cytoskeletal linker protein Gephyrin, and ATPase Nethylmaliemide-sensitive factor (NSF) (that activates SNARE proteins) to the synaptic sites ${ }^{115}$.

These and many more studies add to the evidence that most AZ proteins and synaptic proteins originate from the TGN ${ }^{96,113,115116-118}$, although no known link between cis-Golgi traffic and supply to the presynaptic terminus has been documented. Nevertheless, proteins undergoing a first round of sorting at the cisGolgi are known to be either internally trafficked, through all the Golgi lamellae, to the TGN for a second round of sorting or a subset of certain proteins are also known to be transported back to the ER after only the first round of sorting ${ }^{119}$.

Interestingly, glutamate receptor NMDAR subtypes and their associated postsynaptic adaptor proteins CASK and SAP97 have been shown to be transported from the cis-Golgi and localized at the ER. From the ER these proteins take an unconventional secretory pathway to the plasma membrane where the proteins first get trafficked to axonal and dendritic Golgi outposts, which eventually traffic these proteins to presynaptic and post-synaptic sites ${ }^{120}$.

As Munc13-1 is the first documented AZP to be localized at the cis-Golgi and does not colocalize to the TGN, it might take a similarly unconventional route, like the NMDAR receptors, out of the soma to the presynapse (Figure 28). This choice of unconventional localization may hint at a mechanism that brings a 
greater diversity of proteins to the presynapse, temporally recruits subsets of AZPs for each step of the CAZ assembly, and fosters heterogeneity within preassembled AZP subsets co-trafficking on small vesicle clusters.

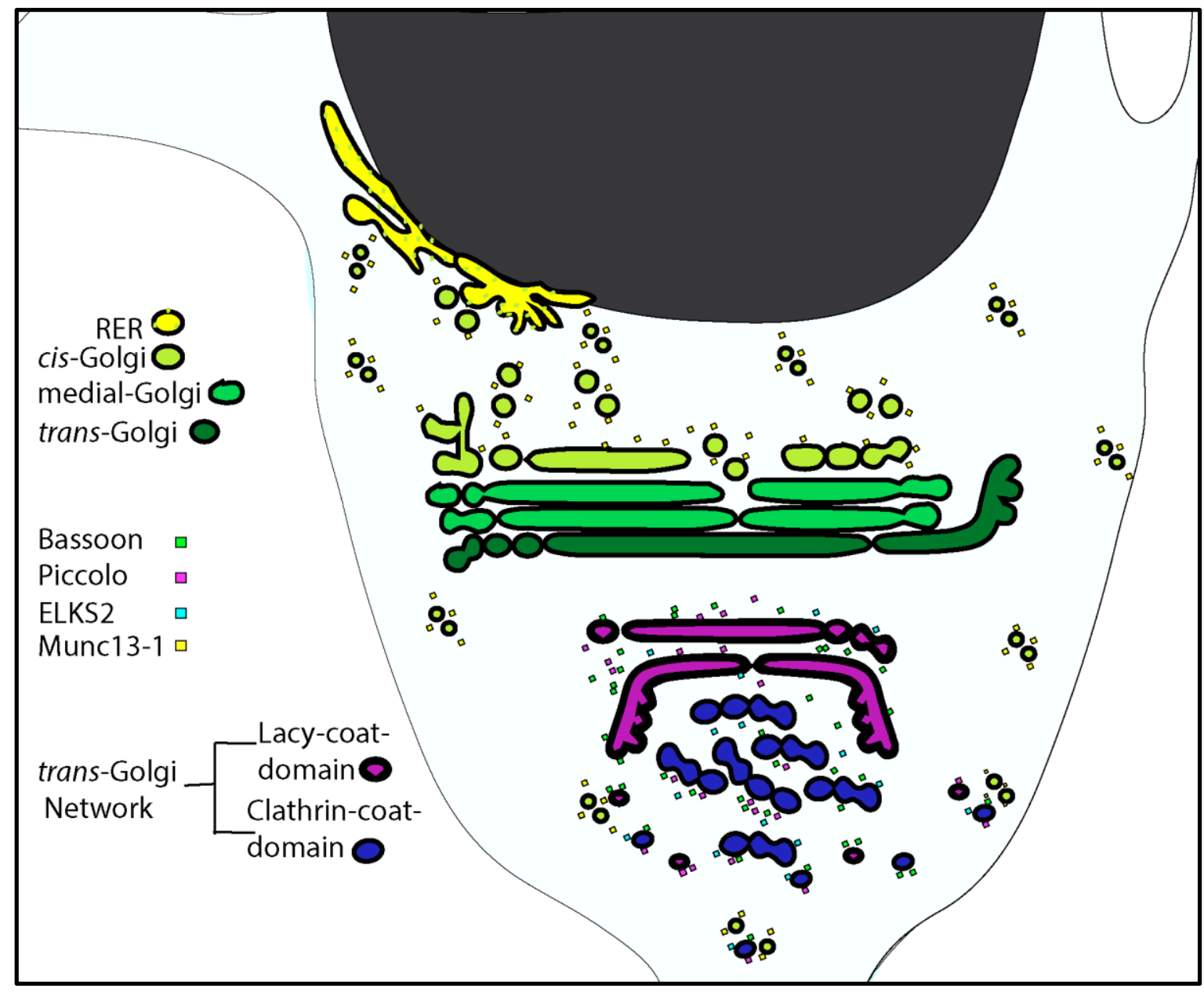

Figure 28: A diagrammatic representation of AZPs localizations to different Golgi subcompartments. This diagram depicts a schematic of transport precursors being generated at the cis-Golgi vesicles carry Munc13-1 and TGN vesicles carrying subsets of ELKS2, Piccolo and Bassoon which may come in close proximity in the soma for co-transport down the axon.

\subsubsection{The N-terminus of Bassoon localizes it to the TGN}

The localization of Bassoon, Piccolo and ELKS to the TGN may be conferred by a direct interaction site on the protein or via an adaptor protein that is localized at the TGN lamella. N-terminally tagged RFP-Bsn specifically localizes to the TGN marker TGN38 but not to the trans-Golgi lamella marker $\beta$-1,4galctosyltransferase 1 , positioning full-length Bassoon to the sorting TGN compartment (Figure 16). The C-terminus of full-length Bassoon does not localize close to the TGN lamella but also does not localize at the trans-Golgi lamella (Appendix F). Therefore Bassoon molecules are not localized between the transGolgi lamella and the TGN but are rather localized on a more distal Golgi lamella.

The TGN network constitutes the last three cisternae of the Golgi and has subdomains that are characteristic for different resident proteins. TGN cisternae 5 
and 6, of the whole Golgi stack, are asialoglycoprotein positive and are decorated with $60 \mathrm{~nm}$ clear-core vesicles with lace-like-coats that are regulated by protein kinase $\mathrm{D}$ activity. These vesicles are believed to transport cargoes en route to the plasma membrane. On the other hand, mannose-6-phosphate protein positive cisternae 7 is known as the clathrin-coat subdomain of the TGN, and produces $\sim 100 \mathrm{~nm}$ clathrin-coat buds that are regulated by ADP-ribosylation factor family of GTPases (ARFs) and GGA (Golgi-localized, gamma-ear containing, ADPribosylation factor binding) proteins ${ }^{121,122}$.

Since neither full-length Bassoon termini are localized close to the trans-Golgi lamella (Figure 16, Figure 17-Figure 19, and Appendix F) and endogenous Bassoon majorly presents itself in signal sizes of $95-150 \mathrm{~nm}$ (Figure 5), it seems likely that Bassoon molecules may be localized to the furthest TGN subdomain of cisternae 7. In addition, Bassoon transported on a range of different DCVs subtypes such as CGA-positive vesicles, PTVs and Syt4-positive vesicles (Figures 6, 25 and ref ${ }^{69}$ ), which may also possess clathrin coats, since there is strong evidence from electron micrographs of neuroendocrine cells demonstrating budding DCVs possessing clathrin coats ${ }^{123,124}$. It therefore would be interesting to see if Bassoon carrying PTV transporters are clathrin-coated and whether their fission from the TGN lamella is ARF- or GGA-protein mediated.

Full-length Bassoon is highly colocalized to TGN markers through its $\mathrm{N}$-terminus and not though its C-terminus (Figure 16-Figure 19, and Appendix F), making the $\mathrm{N}$-terminus of Bassoon its localization and possibly interaction domain for sorting at the TGN. The myristoyl motif, at the second amino-acid position of the $\mathrm{N}$-terminus, was found to not influence its localization at the TGN though another site within the $\mathrm{N}$-terminal domain might (Figure 23). This would require a systematic characterization of an array of $\mathrm{N}$-terminal deletion constructs to pinpoint the exact site that influences Bassoon's localization, although it might very well be possible that the $\mathrm{N}$-terminus of Bassoon never integrates into the TGN membrane but rather is attached to the TGN via an adaptor protein or is directly loaded onto constitutively formed nascent buds from the TGN lamella.

\subsection{Recruitment and sorting of AZPs at the TGN}

\subsubsection{The role of the $\mathrm{CC} 2$ domain in recruiting Bassoon to the Golgi}

The Rbb26-Bsn construct spans a 475-a.a sequence (2088-2563 a.a.) and contains the centrally localized coil-coil 2 (CC2) domain of Bassoon. This domain has been reported as the hetero-/homo-oligomerization domain of Bassoon and the protein has been seen accumulated to the Golgi. Recombinant constructs containing the $\mathrm{CC} 2$ domain of Bassoon are subsequently referred to possessing "the Golgi Binding region of Bassoon"30,32,70. However, in the studies here, I have 
resolved Rbb26-Bsn signals with STED and see them primarily localized at an average distance of $171.9 \mathrm{~nm} \pm 34.8 \mathrm{~nm}$ SD from the TGN membrane markers (Figure 22), while full-length RFP-Bsn signals are localized closer, at an average distance of $50.5 \mathrm{~nm} \pm 8.5 \mathrm{~nm} \mathrm{SD}$, to the TGN membrane markers (Figure 18). This result reveals that the CC2 domain of the Bassoon molecule is in fact not the site within the Bassoon molecule that binds Bassoon to its Golgi compartment.

As the signals of the $\mathrm{N}$-terminal deletion construct 95-Bsn have a similar distance distributions like Rbb26-Bsn and an average distance of $157.1 \mathrm{~nm} \pm 40.5 \mathrm{~nm}$ SD from the TGN (Figure 22), it might be possible that both Rbb26-Bsn and 95-Bsn deletion mutant proteins are mislocalized to another Golgi compartment, as a result of the loss of the $\mathrm{N}$-terminal region. This observation in combination with the high colocalization of N-terminally tagged Bassoon molecules to the TGN (Figures 16-19), show that $\mathrm{N}$-terminus of Bassoon rather than the central CC2 domain may possess the TGN binding domain.

Nonetheless, it is quite remarkable that this small piece of Bassoon, lacking both $\mathrm{N}$ - and C-termini, localizes the mutant protein to the Golgi, even though it might be mislocalized to the wrong subcompartment. This indicates that the CC2 domain of Bassoon recruits Bassoon and possibly Piccolo molecules (which also have a similar CC2 domain), from the somatic cytoplasm to the Golgi.

\subsubsection{Potential sorting mechanisms of AZPs}

Sorting of membrane proteins typically occurs at the TGN. Sorting of proteins at the TGN is accomplished by the combination of a passive process and active process at the TGN. DCV proteins undergoing sorting at the TGN tend to first passively accumulate and form aggregates around the TGN lamella, as a result of the high $\mathrm{Ca}^{2+}$ concentration promoted around the TGN lamellae. These aggregated proteins next get packed in or onto TGN transport precursors often with the help of a sorting signal on the protein that enter through the TGN lamella and bind to sorting receptors within the TGN lumen in an active process ${ }^{125}$. Alternatively, membrane-associated DCV proteins are known to directly interact at lipid raft sites in the TGN membrane, which are the cholesterol-sphingolipidrich microdomains, where budding of TGN precursors occurs ${ }^{126}$. As a mutation of, the only identified lipid integrating, myristoyl motif in Bassoon's N-terminus does not alter its localization to the TGN (Figure 23), Bassoon may not be sorted via the active process of directly binding to a lipid raft or a sorting receptor site on the TGN and may become accumulated around the TGN lamella for sorting via a passive process.

The passive influence of high $\mathrm{Ca}^{2+}$ levels is known to regulate the activity of the neuronal calcium sensor-1 protein - Calneuron, which has been reported to inhibit PI4K $\beta$ (phosphoinositide 4-kinase III $\beta$ ) activity at the TGN. PI4K $\beta$ and elevated $\mathrm{Ca}^{2+}$ levels have been show to mediates the synthesis of phospholipids 
required for the budding of PTVs and synaptophysin-positive vesicles (SPVs) and influence their exit from the TGN ${ }^{127}$.

This suggests that similar sorting conditions that apply to DCV proteins may also apply to Bassoon, Piccolo, and ELKs, the cargo for the PTV subtype of DCVs, in which Bassoon may accumulate at the TGN due to passive sorting conditions such as the high $\mathrm{Ca}^{2+}$ concentrations around the TGN, form AZP clusters with neighboring Bassoon and Piccolo molecules via its $\mathrm{CC} 2$ oligomerization domain and be loaded onto PTVs and SPVs via PI4K $\beta$ activity.

Much less is known about the mechanisms by which AZPs at the cis-Golgi are sorted. Although, COPI-coated cis-Golgi vesicles transported back to the ER are also sorted in a $\mathrm{Ca}^{2+}$-dependent manner ${ }^{127}$. Munc13-1 vesicles that may take the unconventional NMDARs pathway out of the soma may also undergo a similar $\mathrm{Ca}^{2+}$ mediated passive sorting step at the cis-Golgi, which may promote accumulation of Munc13-1 to be loaded onto a range of transport carriers.

These sorting mechanisms support the observations of large populations of AZP signal sizes corresponding to small vesicle cluster sizes, seen in the neuronal soma with STED imaging (Figure 5), and promote the idea that subsets of AZPs get loaded on transport carriers and form a partially preassembled set of proteins, before their transport down the axon.

As AZP signals were observed in a range of signal sizes in the soma ( Figure 5), these proteins may be loaded onto a range of different transport vesicle types, which may in turn require different sorting and vesicle fission mechanisms.

This notion may very well be feasible, as Bassoon's localization to the TGN is also proposed to be mediated by the carboxyl-terminal binding protein (CtBP) family. The N-terminus of CtBP-1 is known to bind to Bassoon at a site directly upstream of its $\mathrm{CC} 2$ domain at the Golgi and has also been shown to inhibit Bassoon and Piccolo transport into axons in CtBP1 loss of function experiments ${ }^{26,32}$. CtBP-3, an ortholog of CtBP1, is involved in mediating vesicle fission at the Golgi ${ }^{128}$ and may be involved in the sorting and fission of a certain subgroup of Bassoon and Piccolo carrying transport precursors at the TGN.

Additionally, invertebrate-specific AZP protein Liprin- $\alpha$ is known to interact with GTPase-activating proteins (GITs) subtype of ARFs that regulate its fission and traffic out of the TGN on CAST/ELKS positive vesicles ${ }^{111,129,130}$. As ARF mediated vesicle formation is favored at cisternae $7^{121}$, the last TGN lamella, it is likely that AZP proteins are preferentially sorted at cisternae 7 and loaded onto varying types of transport vesicles that may employ a specific sorting mechanism whether it is mediated via ARFs, CtBPs or PI4K $\beta$. 


\subsection{Transport of AZPs from the soma to nascent presynaptic sites}

Pioneering studies in the field have shown AZPs Bassoon and Piccolo to be localized in the somas of young neurons at Golgi substructures and transported to presynaptic sites on $80 \mathrm{~nm}$ dense-core vesicles known as Piccolo-Bassoon Transport Vesicles (PTVs) ${ }^{30,68,69}$. Other AZPs, namely Munc13-1, RIM1 $\alpha$, and ELKS2, and synaptic proteins syntaxin-1, SNAP25, N-cadherin, Rim1, and Chromgranins were also isolated in light brain fraction immunoprecipitates containing PTVs ${ }^{69,33,68}$. Golgi-derived AZP transport carriers, which are either preassembled $\mathrm{PTVs}{ }^{93}$ or small clusters of clear- and dense-core vesicles carrying $\mathrm{AZPs}^{71}$, are believed to sufficiently transport all AZPs to the presynaptic membrane, fuse with the membrane and leave behind an entire complement of proteins that generate the CAZ scaffold and integrate with the presynaptic machinery to form a functional synapse $e^{9,68,69}$.

The current state of studies shows the existence of at least three types of specialized transport precursors that carry complexes of Piccolo-BassoonELKS/CAST ${ }^{3,32,71}$, RIM-Neurexin-CASK-VGCCs ${ }^{3}$, and Munc13-1 ${ }^{32}$ to mammalian synaptic sites. These subsets of AZPs trafficking to nascent synaptic sites use the microtubule-based transport system of the neuron by interacting with the anterograde and the retrograde motors moving along the microtubules in the axon $^{29,32,131}$. Bassoon and Piccolo containing PTVs are transported anterogradely via the PTV specific adaptor syntabulin that binds to the KIF5b motor protein ${ }^{72,95}$, and also traffic in the retrograde fashion facilitated by Bassoon's direct binding to dynein light chains (Dlc1/2), a retrograde motor ${ }^{29}$. Conversely, the invertebrate specific variants of RIM and Munc13 proteins, loaded on to synaptic vesicles (SVs), are only transported in an anterograde fashion with the help of kinesin motors $^{132}$.

Co-trafficking of PTVs and small clear core vesicles, reminiscent of SVs, have been reported in ultra-structural studies of hippocampal axons. Electron microscopy (EM) images from this study show 220nmX130nm transport aggregates consisting of one to two CGA-positive PTVs and up to five SVs that express a range of SV proteins such as VAMP2, synaptotagmin, synapsin-1 and SV2, co-trafficking together ${ }^{71}$.

These immunogold labeled EM images of co-trafficking AZPs and SV proteins in vesicle aggregates showed Bassoon and Piccolo signals typically on top of DCVs with their signals clustered in the center of the aggregates.

These results corroborate my own data, in which $\mathrm{N}$ - or C-terminally tagged fulllength Bassoon were observed localized on top of a CGA positive vesicle. In addition, the $\mathrm{N}$ - and $\mathrm{C}$-termini of full-length Bassoon appear equidistant from the 
CGA positive vesicle core with almost no colocalization between the Bassoon and CGA signals (Figure 25). These results reflect that a group of Bassoon molecules co-traffic with CGA positive vesicles, most probably as a part of a vesicle cluster and Bassoon molecules do not retain their extended orientation and organization on top these transport carriers as their $\mathrm{N}$ - or C-termini are equidistantly positioned in the transport cluster. These transport entities are therefore arranged to efficiently co-traffic a subset of AZPs and SV proteins in a compact form to nascent synaptic sites.

Such co-trafficking entities possessing different vesicle types may arise from different Golgi substructure and may serve the purpose of bringing different subsets of AZPs in close proximity of each other, for optimal delivery of all AZPs to nascent synaptic sites.

In this study, I show with STED imaging that AZPs have a much lower colocalization with each other than previously assumed, in comparison to cotrafficking events of AZPs recorded with confocal microscopy (Figure 8). In addition, the distribution of AZP colocalizations at proximal, central, and distal axonal regions in DIV6 neurons is uniform (Figure 7).

This small population of co-trafficking vesicles in axons points not only to the lower possibility of co-trafficking events of AZPs being coordinated during transport but also suggests that sorting mechanisms in the soma may play a larger role in preassembling subsets of AZPs and their vesicle clusters for direct delivery to nascent synaptic sites.

RIM1 $\alpha$ and PSD95 are synaptic proteins that are transported both via active and passive mechanisms to synaptic sites and are present in the soluble pools during early development ${ }^{32,133}$. Both these proteins can be recruited from the soluble pools and appear associated later during development on their respective transport carriers ${ }^{3,133,134}$. A similar mechanism has been proposed for the preassembly of a complete AZP set on to PTVs, that don't start out carrying all AZPs when they leave the Golgi but acquire them en route, and is also suggested as a mechanism for maturing Golgi derived transport precursors into matured PTVs that supply a sufficient subset of all AZPs to the presynaptic membrane ${ }^{32}$.

However, since the distribution of the AZPs and their colocalizations with one another remain uniform in the developing axon (Figure 7), it seems more likely that transport vesicles arrive at the presynaptic terminus consecutively and supply the full complement of AZPs required to form the CAZ in a temporal fashion. This notion supports observations that show PTVs carry unitary amounts of AZPs and predicts that on average the cargo of 2-3 PTVs may be required to assemble a complete $A Z^{69}$. 
Through their interaction with anterograde and retrograde motor complexes, AZPs on DCVs have been proposed to constantly circulate along axons and supply nascent synaptic sites and new en passant boutons of invertebrate neurons ${ }^{135}$. This constant and dynamic access of transport packages to the CAZ could be a mechanism by which neurons temporally and spatially control the assembly and maturation of the CAZ structure ${ }^{74}$.

Interestingly, hierarchical assembly mechanisms have already been observed in C. elegans and Drosophila neurons, in which transmembrane proteins SYG-1/2 initiate synaptogenesis by first recruiting the key scaffolding proteins SYD-2/liprin$\alpha$ and SYD-1, which in turn assemble the other presynaptic components ${ }^{19,136}$.

It therefore seems ideal, that at mammalian presynapses, the structural scaffolding proteins Bassoon and Piccolo are the first recruits that form the CAZ backbone. These proteins are delivered off of PTVs co-trafficking in clusters with SVs previously observed ${ }^{71}$, that also bring along ELKS2 and a small subgroup of SV proteins and may together suffice the initiation of a nascent CAZ at presynaptic sites ${ }^{71,131}$. This nascent structure containing the oriented molecules of Bassoon and Piccolo may in turn then recruit their C-terminal binding partners Rim1 $\alpha$ and Munc13-1 from their trafficking vesicle subtype and/or from the soluble cytoplasmic pool.

\subsection{Orientation of the Bassoon molecule}

\subsubsection{Current concept: Bassoon possesses an extended conformation at the presynaptic terminus}

Bassoon $(420 \mathrm{kDa})$ and Piccolo $(550 \mathrm{kDa})$ are the two largest proteins in the CAZ, and both molecules have been shown to possess an extended orientation at matured presynaptic sites in mammalian neurons ${ }^{76,77}$. In-silico modeling predicts that both molecules contain a large number of proline and glycine residues, which prevent folding of these proteins as a whole, but also contain interspersed compact regions that hold the $\mathrm{Zn}$-finger, coil-coil, and Piccolo-Bassoon homology domains, as a result of which their entire molecule elongates to about $80 \mathrm{~nm}$ (Figure 29,B and $\mathrm{ref}^{24}$ ). 


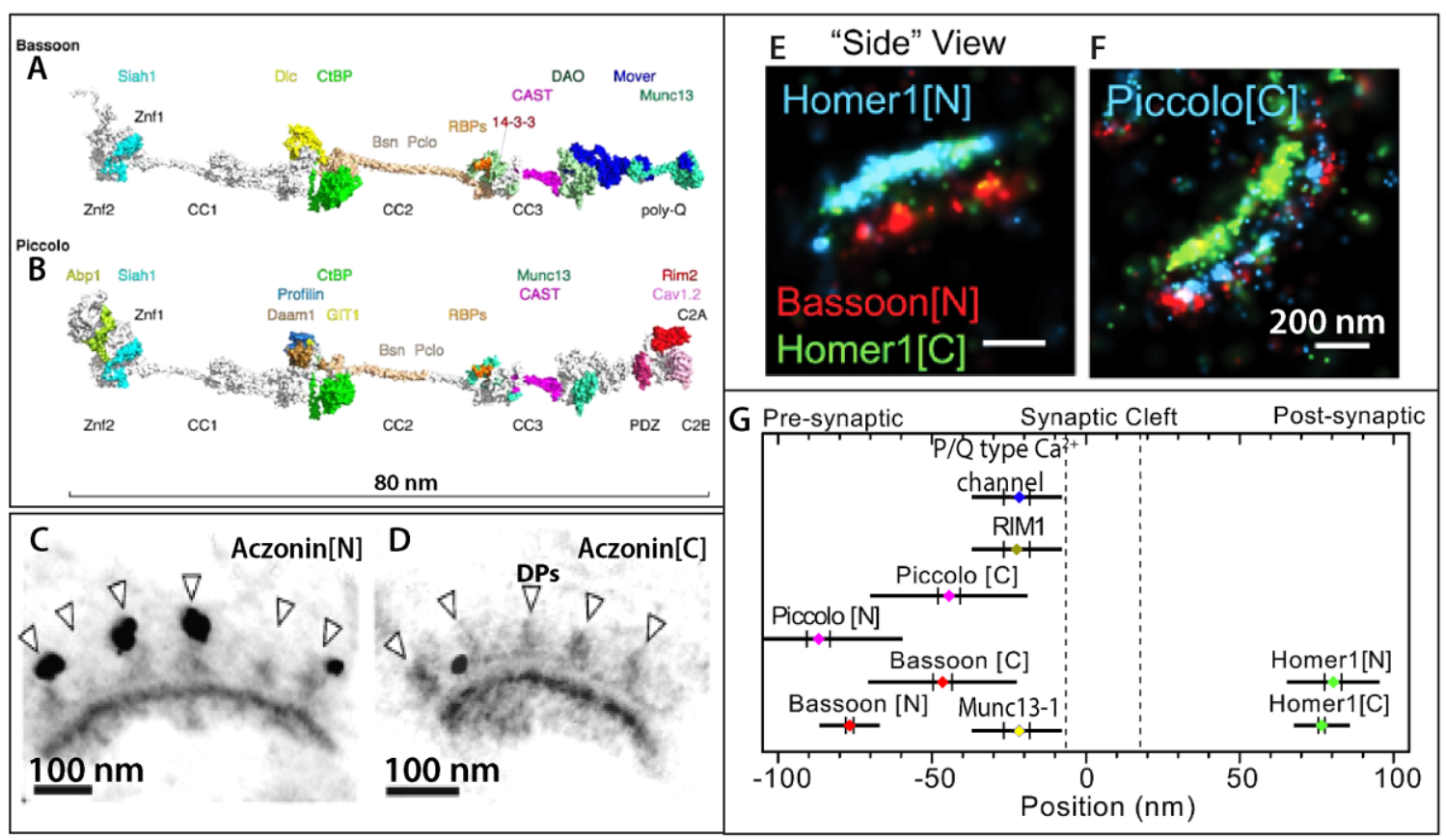

Figure 29: Structure and orientation of Bassoon and Piccolo proteins at the presynaptic terminus. $\mathbf{A}$ and $\mathbf{B}$, In-silicio model of Bassoon and Piccolo proteins with interaction sites and binding partners (Modified from Gundelfinger et al, 2015). C and D, Gold labeled EM images of N- and Ctermini of Piccolo protein, on the apical and base locations of the dense projections (DPs) in mammalian presynaptic sites (Modified from Limbach et al, 2011). E and F, represent triple colour-3D STORM images of Bassoon and Piccolo at side view synapses (Modified from Dani et al, 2010). $G$ is a representation of CAZ and pre- and postsynaptic protein distances from the synaptic cleft. (Modified from Dani et al, 2010 and enhanced with information on Munc13-1, RIM1 and $\mathrm{Ca}^{2+}$ channel distances from Limbach et al, 2011).

In a recent study, triple-color 3D STORM images of cortical synapses labeled using a Bassoon monoclonal antibody spanning the region between 7651001a.a. (closest antibody to the N-terminus of Bassoon), a C-terminal polyclonal Bassoon antibody spanning the last 338 a.a. of the molecule, and a Homer1 antibody (to define postsynaptic membrane and the synaptic cleft size) were used to determine Bassoon's orientation at the synapse (Figure 29E,F). These images showed that the N-terminus of Bassoon is localized at $\sim 78 \mathrm{~nm}$, and $\sim 30 \mathrm{~nm}$ further away from the synaptic cleft than the C-terminus of Bassoon signals, which is localized $\sim 43 \mathrm{~nm}$ from the synaptic cleft. Bassoon and Piccolo signals were shown to spatially occupy similar locations in the CAZ as Piccolo's N- and Cterminal regions were located roughly $86 \mathrm{~nm}$ and $44.4 \mathrm{~nm}$, respectively, from the synaptic cleft ${ }^{76}$.

The orientations of Bassoon and Piccolo molecules recorded by STORM imaging also fit with results from another study that studied the localization of many immunogold electron micrographs of cerebellar rat neurons labeled for a range of antibodies generated against short sequences within Aczonin/Piccolo molecule and for Bassoon's C-terminus. $70 \%$ of the total N-terminal sequences of Piccolo were observed localized within $73-79 \mathrm{~nm}$ from the plasma membrane (PM), 
while the $70 \%$ of the C-terminal Piccolo sequences lay within 33-39nm, and Bassoon's C-terminal antibody signals were observed positioned within 35$37 \mathrm{~nm}$ from the PM (Figure 29C,D and $\mathrm{ref}^{77}$ ).

Both these studies, using two different super-resolution imaging techniques, have shown that the N-termini of Bassoon and Piccolo molecules within an AZ complex are at least $80 \mathrm{~nm}$ from the PM, their C-termini face the PM, and are roughly $30-40 \mathrm{~nm}$ from the presynaptic membrane.

Ultrastructural imaging of the same synapses also revealed AZPs that bind to Ctermini of Bassoon and Piccolo, in particular the N-termini of Munc13-1 and RIM1 $\alpha$, localized $19 \mathrm{~nm}$ from the PM and the cytoplasmic loop of the P/Q type $\mathrm{Ca}^{2+}$ channels were localized 20nm from the PM, positioning the entire $\mathrm{AZ}$ complex within a minimum distance range of $20-80 \mathrm{~nm}$ from the PM (Figure 28G and $\operatorname{ref}^{77}$ ). These studies revealed a range of additional characteristics that define the CAZ ultrastructure such as the following: the presence of $60 \mathrm{~nm}$ high and $30 \mathrm{~nm}$ wide dense projections (DPs) at the presynapses of chemically fixed EM images that reflect the dimensions of a collapsed CAZ filament structure (Figure $28 \mathrm{C} / \mathrm{D}$ and $\mathrm{ref}^{77}$ ), the distances of the C-termini of Piccolo and Bassoon noted are similar to the diameter of synaptic vesicles (SVs) (i.e. $\sim 35-40 \mathrm{~nm})^{3,77}$, and that none of the AZPs in the CAZ have transmembrane regions that warrant their presence at the $\mathrm{PM}^{40,70}$. This shows that the CAZ structure localizes within the presynaptic bouton but floats at a minimum $20-30 \mathrm{~nm}$ distance, reflective of the size of a SVs docked, on top of the PM.

This extended orientation of Bassoon and Piccolo molecules seem to inherently influence the function of the CAZ, and are also seen in invertebrate synapses. Bruchpilot, a Drosophila CAZ core component that is evolutionarily unrelated to Bassoon and Piccolo, has also been shown to possess an extended orientation at the neuromuscular junction. Although the orientation of Bruchpilot differs from Bassoon and Piccolo's, as its N-terminus is closer to the presynaptic membrane, it vertically extends $40-65 \mathrm{~nm}$ into the presynaptic bouton and matches Bassoon and Piccolo heights in mammalian presynaptic membrane ${ }^{77,137}$. This shows that CAZ structure needs to be accessible to resting and recycling SV pools in the presynaptic terminus, and Bassoon and Piccolo, like Bruchpilot at invertebrate synapses, provide the structural backbone of the CAZ scaffold with their extended conformations.

\subsubsection{Bassoon also possesses an extended conformation at the TGN but it is inversely oriented}

In this study, Bassoon was visualized at its first station the TGN and was revealed to possess an extended conformation at this structure. This is a novel observation as it ties the orientation of Bassoon at it its initial and final station in its journey, shows that Bassoon possess a similar conformation at both sites and 
that the orientation of the molecule is inversed during or after its transport to synaptic sites.

I show that $67 \%$ of N-terminal tag of the full-length RFP-Bsn-GFP construct is localized on average $32.5 \mathrm{~nm} \pm 8 \mathrm{~nm}$ SD from the closest TGN38 marker, while $33 \%$ of $\mathrm{N}$-terminal tag of the same construct was localized on average at $229.4 \mathrm{~nm} \pm 5.3 \mathrm{~nm}$ SD from the closest TGN38 marker. Meanwhile, $63 \%$ of the tagged C-termini signals of the same full-length construct were localized on average around $210.6 \mathrm{~nm} \pm 13 \mathrm{~nm} \mathrm{SD}$, and the remaining $37 \%$ of C-terminal tag signals were localized around $60.4 \mathrm{~nm} \pm 6.8 \mathrm{~nm}$ SD from the TGN38 marker. This predicts that at and around the TGN, there is a mixed population of Bassoon molecules that are being oriented at the TGN and those that are already loaded onto transport carriers. The closest signals of full-length Bassoon's N-terminal tag were seen localized $\sim 30-40 \mathrm{~nm}$ from the TGN and the closest C-terminal signals were seen around $60-67 \mathrm{~nm}$ from the TGN membrane. These represent the molecules that are being oriented at the TGN and show that the $\mathrm{N}$ - and C-termini of Bassoon are positioned within 30-40nm of each other (Figure 17). These results remarkably concur with the $30-40 \mathrm{~nm}$ extension of the Bassoon molecule seen in mammalian presynaptic boutons, from their EM and STORM orientation studies $^{76,77}$.

The second fraction of Bassoon molecules were seen oriented within 210$229 \mathrm{~nm}$ from the TGN membrane and may reflect the populations of Bassoon molecules that are already loaded onto transport carriers. It is interesting that both the $\mathrm{N}$ - and $\mathrm{C}$-termini of Bassoon molecules in the second fraction are roughly equidistant from the TGN lamella and confirm the observation of Bassoon's equidistant localization on top of CGA-positive DCVs, seen further down the axon (Figure 25 and Figure 17).

These localizations of full-length Bassoon are extremely reproducible and robust since similar distances for single-tagged RFP-Bsn and Bsn-RFP constructs were also observed, along with a similar distribution of all full-length Bassoon constructs to a different TGN marker: Syn6 (Figures 18 and 19). The subset of single-tagged Bassoon signals being orientated at the TGN were localized at $30.5 \mathrm{~nm} \pm 6.9 \mathrm{~nm} \mathrm{SD}$ (for $85 \%$ of the RFP-Bsn) and at $50.3 \mathrm{~nm} \pm 3 \mathrm{~nm}$ SD (for $45 \%$ of the Bsn-RFP) from the closest TGN marker, while the fraction of signals on transport precursors were localized at $154.1 \mathrm{~nm} \pm 12.06 \mathrm{~nm}$ SD (for $15 \%$ of RFPBsn) and $192 \mathrm{~nm} \pm 11.6 \mathrm{~nm}$ SD (for $55 \%$ of the Bsn-RFP) (Figure 18).

Overall these results show that Bassoon orients itself with its $\mathrm{N}$-terminus at the TGN membrane while its C-terminus is at least $30-40 \mathrm{~nm}$ form the TGN lamella. Although the extension of the Bassoon molecule is remarkably similar to its reported extension within the CAZ, the orientation of the molecule is inversed between the two stations. As the Bassoon molecule does not possess transmembrane regions that may integrate the molecule at either the TGN lipid 
bilayer or the PM, and its $\mathrm{N}$-terminal myristoyl motif is not involved with its localization to the TGN membrane, one may assume that Bassoon's orientation is not influenced by a docking site within either membrane at the two stations. The inverse orientation is therefore a reflection of the roles and the local subcellular mechanisms the Bassoon molecule is involved in, at both stations.

At the TGN, the extended orientation of Bassoon may promote sorting mechanisms and aid in exposing Bassoon's central CC2 domain. The CC2 domain has been shown to sufficiently recruit Bassoon molecules to the Golgi (Figure 22), and, while at the TGN, promotes oligomerization of BassoonBassoon and Bassoon-Piccolo clusters that get loaded onto a range of transport carriers thereby promoting subsets of preassembled AZP to be co-trafficked. Additionally upstream from the CC2 domain lies the CtBP1 interaction site, which should become available for binding as Bassoon's conformation becomes extended at the TGN membrane thereby promoting CtBP1 mediated sorting. It therefore would be interesting to visualize the average localization that CtBP1 occupies at the TGN using full-length Bassoon as a ruler.

\subsubsection{The limitations of different super-resolution microscopy techniques in understanding Bassoon's orientation}

Until recently, all ultrastructural imaging in the brain was done using transmission electron microscopy (TEM). The constant challenge of acquiring sufficient contrast though ultrathin sectioning, followed by the demands of serial sectioning and section alignment, have decreased the throughput of TEM sample preparation. In addition, the technique necessitates harsh sample preparations that often subject the samples to form artifacts that could obscure the true structure and localization of proteins at an ultrastructural level. The last decade has seen the rise of light microscopes created to break the resolution limit of light and resolve a much higher level of detail. STED and STORM microscopy are classical examples of such tools that have created a platform to revisit and add to existing EM data by allowing the visualization of tagged proteins at superresolution.

In this study, I have made use of a range of full-length Bassoon constructs that have allowed me to visualize the orientation of the Bassoon molecule with great detail and accuracy. Visualizing these constructs with two-color STED microscopy (at a resolution limit of 20nm) benefits from the tags on the $\mathrm{N}$ - and $\mathrm{C}$ termini of Bassoon being placed at the extreme poles of the protein, without hampering its localization, transport and incorporation into synapses, and allows for the use of specific nanobodies targeting the fluorescent tags, that localize the whole tag-complex within $13 \mathrm{~nm}$ of the protein. The use of the double-tagged fulllength Bassoon construct, boosted with nanobodies, and imaged with STED to visualize the localization of the $\mathrm{N}$ - and $\mathrm{C}$-termini of Bassoon, overcomes a range 
of disadvantages faced by the other super-resolution imaging studies performed. One of the key advantages is eliminating the use of traditional antibodies of Bassoon for STED microscopy, which would create a $30 \mathrm{~nm}$ cloud of signal around the protein epitope and distances the correct subcellular localization of the termini of Bassoon. STED imaging of transfected neurons also benefits from the ability to avoid the use of the harsh processing steps that are essential for processing EM images that cause the CAZ structure to collapse into DPs. When comparing my imaging paradigm with the drawbacks of STORM imaging results of Bassoon's orientation at the presynaptic termini, it becomes clear that the latter's results suffer from the application of two traditional antibodies targeting a region of Bassoon around its $1000^{\text {th }}$ a.a. and the extreme C-terminal region and can therefore only predict, at a $30 \mathrm{~nm}$ distance from the true localization, the distance between the $1000^{\text {th }}$ and $4000^{\text {th }}$ amino acids of the Bassoon molecule. It therefore would be interesting to visualize the orientation of Bassoon at mature synapses using nanobodies and the full-length Bassoon constructs and ascertain whether the first 1000 a.a. of Bassoon add to further extend the molecule into the presynaptic bouton or whether it folds to create a cap for the filament like structure that Bassoon possesses within the CAZ.

The STED imaging paradigm I use, on the other hand, is limited by the confocal resolution $(\sim 200 \mathrm{~nm})$ in its $z$-plane, in comparison 3D STORM imaging allows for a $\sim 14 \mathrm{~nm}$ resolution limit in the $z$-plane. This limitation has so far made it difficult to find in culture, pre- and postsynaptic sites in the side view conformation, which is a clear separation of pre- and postsynaptic compartments with the synaptic cleft (Figure 28E, F). This conformation of viewing synapses is important as it provides a clear marker from which to define the distance of the $\mathrm{N}$ - and $\mathrm{C}$-termini of a molecule and can be observed with greater ease with 3D super resolution imaging. In addition, to understand the real orientation and organization of CAZ proteins in the CAZ scaffold live neurons need to imaged, for which the required dual-color RFP and GFP live STED microscopes are still being developed.

\subsection{Organization of Bassoon molecules at the TGN and presynaptic sites}

In addition to showing that Bassoon possesses the same extended conformation at the TGN and at the synapses, this study also reveals how Bassoon molecules are organized at both these locations. These novel results allow us to understand how the orientation of one Bassoon molecule interacts with neighboring Bassoon molecules to ultimately contribute to Bassoon's structural functionality at both subcellular locations.

In the soma, the signals of the N-termini of co-transfected RFP-Bsn and GFPBsn, boosted with a GFP polyclonal antibody and an RFP nanobody, were seen packed within $5 \mathrm{~nm}$ and produced a $+3.7 \%$ FRET. On the other hand, C-termini of 
Bsn-RFP and Bsn-GFP, boosted similarly, produced no FRET. This result shows that the $\mathrm{N}$-termini of neighboring Bassoon molecules are closely organized while the C-termini of neighboring Bassoon molecules are at least 6nm apart (Figure 26). This observation most importantly links the role of the $\mathrm{N}$-termini of Bassoon in promoting an extended conformation at the TGN and the organization of neighboring molecules, such that Bassoon molecules may align in close proximity to one another, thereby enabling efficient sorting at the TGN. The organization of the $\mathrm{N}$-termini of Bassoon may also help accentuate the open conformation and orientation of the Bassoon molecule at the TGN, and the cuulative effect of both features should in turn promote oligomerization between neighboring Bassoon molecules and ultimately provide a greater variety of AZP subsets and higher cotrafficking opportunities of AZPs exiting the soma.

As the organization of Bassoon molecules is conceptualized in an environment flooded with large numbers of tagged Bassoon molecules, thereby increasing the possible Bassoon-Bassoon interactions, it would be important to also ascertain whether neighboring $\mathrm{N}$-termini of Bassoon and Piccolo molecules are similarly organized around the TGN and whether this configuration promotes the generation of preassembled PTVs.

Just as the extended orientation of Bassoon is prevalent at both TGN and presynaptic sites, the intermolecular organization of these proteins, around both the structures, is also similar. Punctate sites on matured neurites that represent synaptic sites, containing co-transfected full-length RFP-Bsn and GFP-Bsn constructs produced a $+7.3 \%$ FRET or containing Bsn-RFP and Bsn-GFP constructs produced no FRET, were readily observed without having to boost the RFP and GFP tags. The robust positive FRET seen between the N-termini of neighboring Bassoon suggests that, at these sites, the organization of Bassoon molecules localize the $\mathrm{N}$-termini of neighboring molecules within $5 \mathrm{~nm}$ of each other (Figure 27).

This result mimics the structure of triangular DPs, often seen in chemically fixed EM images, representing the collapsed CAZ structure atop the presynaptic membrane. The $\mathrm{N}$-termini of Bassoon molecules within a single $\mathrm{AZ}$ form the closely localized apex of the DP and the C-termini the spread out to form the base of the structure. The simplest purpose behind this molecular anatomy of Bassoon molecules at presynaptic sites could be to create a structural backbone for the CAZ scaffold to stabilize upon. The larger distances between the C-termini of neighboring Bassoon molecules could therefore physically separate and generate intermittent SV docking sites on the presynaptic membrane.

The $\mathrm{N}$-termini of Bassoon on the other hand seem to have a deeper role in CAZ ultrastructure and their super close proximities $(<5 \mathrm{~nm})$ warrant the presence of an underlying mechanism that promotes its anatomical and functional roles at the CAZ. It has been previously shown that the $\mathrm{N}$-termini of Bassoon and Piccolo 
molecules possess a Siah1 binding site within their N-terminal zinc finger domains. Siah1 is an E3 ubiquitin ligase that promotes local degradation of proteins within microdomains of the presynaptic bouton by poly-ubiquitination. The ubiquitin-proteasome system is thought to regulate neurotransmitter release, SV recycling and/or the clearing of misfolded or aged proteins within presynaptic boutons ${ }^{13,138}$. Bassoon and Piccolo have been shown to locally regulate this system by binding to and downregulating Siah 1 activity and promoting localized structural stability of the CAZ scaffold ${ }^{25}$. The close localization of neighboring Bassoon N-termini might be a result of two N-terminal zinc fingers binding Siah1. Siah1 is known to exist in dimers ${ }^{139}$ and could recruit N-termini of neighboring Bassoon and Piccolo molecules within extremely close proximities at the CAZ to necessitate the complete activity inhibition of Siah1 dimers.

Overall the extended orientation of Bassoon and the close organization of neighboring N-termini of Bassoon molecules at both the Golgi and presynaptic stations show that Bassoon and possibly Piccolo, through their homology domains, prefer an open conformation when they are not in transit on transport vesicles. These observations also imply that the $\mathrm{N}$-terminus of the Bassoon molecule integrates itself into local functional mechanism, while the $\mathrm{C}$-terminus of the molecule promotes the overall anatomical structure that Bassoon is a part of at the different subcellular sites it is present at. 


\subsection{Perspectives and open questions}

Very little is known about nanostructural events involved in the assembly of the mammalian presynaptic CAZ scaffold; a structure that restricts neurotransmitter release to specific sites on the presynaptic membrane known as the active zone (AZ) and regulates its properties. This study investigates the molecular localizations and the ultrastructural configuration of AZPs, in particular Bassoon, at different subcellular locations in its journey to the presynapses. By doing so this study highlights the anatomical functionality of AZPs at different subcellular localizations and the molecular mechanisms they are involved in, that ultimately influence their assembly in the CAZ scaffold.

I show here that AZPs are first localized to Golgi substructures in young neurons, they are distinctively distributed to specialized Golgi compartments and not all AZPs use the same TGN mediated secretory pathway. This compartmentalization of AZPs at the Golgi is the first mechanism that AZPs undergo that ultimately influences their assembly at presynaptic sites. This early segregation of AZPs into subsets suggests the presence of different transport carriers, trafficking mechanisms and a temporal delivery of AZPs to nascent synaptic sites. I also show that during trafficking the distributions of AZPs are uniform all throughout the axon, which reduces the chances of exchanging or acquiring a higher AZP composition closer to synaptic sites. In addition, upon resolving the confocal colocalizations of AZPs in the axon, it became evident though this study that a remarkably small population of AZPs co-traffic together in the axon, therefore indicating that the assembly of AZPs onto transport precursors in the soma may have a larger influence on the composition of cotrafficked AZPs.

AZPs Bassoon and Piccolo have been identified on transport aggregates containing PTVs and SVs, with a range of SV and DCV proteins, present primarily in young (DIV3-6) axons, that are believed to sufficiently form new functional $\mathrm{AZs}^{71}$. Multiple tags of $\mathrm{N}$ - and $\mathrm{C}$ - terminally tagged full-length Bassoon localized on top of similar vesicles clusters containing CGA positive vesicles trafficking in young axons, were seen in this study with STED imaging, carrying Bassoon molecules in a compact configuration.

All these observations highlight that AZP assembly mechanisms involve a sequential delivery of transport packages, with Bassoon and Piccolo carrying preassembled multi-vesicle transport aggregates arriving first at the nascent presynaptic bouton, followed by the acquisition of RIM and Munc13-1 proteins that take a different transport pathway to presynapse.

In addition, this study identifies a novel configuration change of the large Bassoon molecule, from an open and extended orientation with its $\mathrm{N}$-terminus 
facing the TGN, to a more compact configuration on transport vesicles, and to finally possessing a similar extended orientation at matured presynaptic sites (Figure 30). This change in configuration is an additional mechanism that could necessitate proper transport, assembly and maturation of the CAZ scaffold.

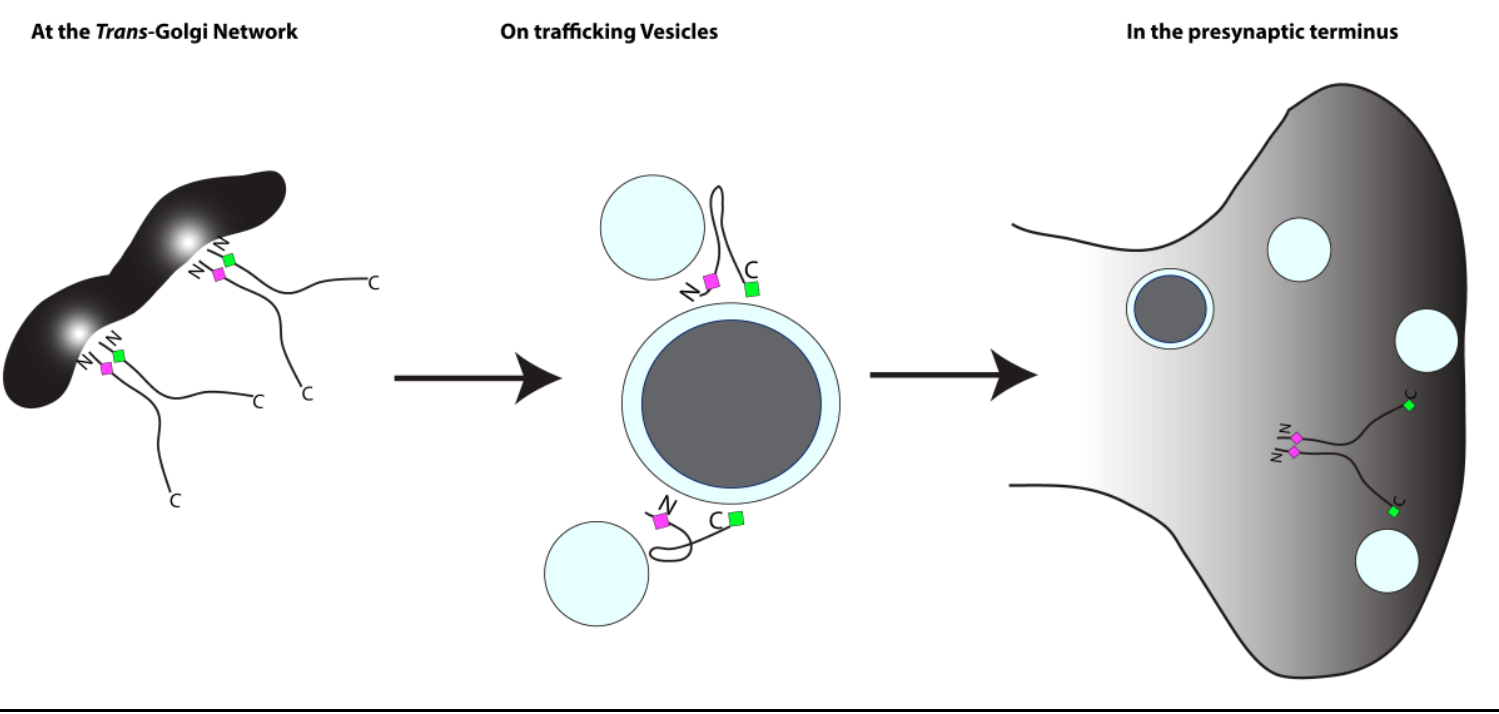

Figure 30: A diagrammatic representation of the orientation and organization of tagged Bassoon molecules. Full-length double- and single-tagged Bassoon molecules are illustrated as they are organized at the TGN lamella, on PTVs, and at their final localization in the presynaptic terminus.

Finally, the orientation of Bassoon molecule also revealed the importance of Bassoon's N-terminus in localizing it to the TGN and accentuating its extended orientation by organizing the $\mathrm{N}$-termini of neighboring Bassoon molecules in close proximity. The $\mathrm{N}$-termini of Bassoon molecules at synaptic sites were also organized within $5 \mathrm{~nm}$ of each other and highlight the role Bassoon's $\mathrm{N}$-termini in inhibiting Siah1-dimer mediated local degradation and confers structural stability in microdomains around the CAZ. The C-termini of Bassoon molecules on the other hand have a less clear role in local mechanisms at the TGN, where they promote the extended conformation of Bassoon by being localized further away from the TGN lamella, and may thereby promote access to the oligomerization domain of Bassoon, enabling Bassoon-Bassoon and/or Bassoon-Piccolo preassembly in the soma for co-traffic on multi-vesicle aggregates. Nonetheless, the C-termini at presynaptic sites avoid close interactions and have been recorded $20-30 \mathrm{~nm}$ from the PM suggesting that the C-termini may help separate two synaptic vesicle docking sites on the PM and form the base, of the CAZ scaffold, to which other AZPs such as Munc13-1 and RIM1 $\alpha$ can bind.

These studies bring forward the first few novel mechanisms that influence mammalian CAZ assembly and provides a platform to understand an integrated model of the molecular localizations, ultrastructural configuration and the anatomical functionality of AZPs from the TGN to the CAZ scaffold. 
As these are the first insights in mammalian CAZ assembly a number of questions are yet to be answered, for example the following:

- What are the sorting and transport mechanism required for cis-Golgi mediated transport precursors of Munc13-1 and when is RIM1 $\alpha$ first associated to the other AZP complement?

- Are preassembled multi-vesicle aggregates assembled at the TGN, in the soma before axonal transport or during transport in developing neurons?

- None of the mammalian AZP proteins have transmembrane regions, yet they use the secretory pathway and are transported to AZ sites where they float on top of the PM. What anchors the CAZ and what factors determine its localization opposite postsynaptic scaffolds?

- Do PTVs and SVs fuse with the PM to deliver AZPs to the presynaptic scaffold?

- How are the other AZPs organized within the CAZ ultrastructure?

- Does the orientation of Bassoon molecule change with activity and is the CAZ scaffold a dynamic structure?

Addressing these questions will not only help understand how the mammalian AZ is assembled and how its structure maintains local synaptic mechanisms but will also improve our fundamental understanding of how basic cellular mechanisms of sorting, transport, and organizations of proteins, that travel long distances to form synapses, are mediated in neurons. 


\section{References}

1. Foster, M., Sherrington, C. S. \& University College, L. L. S. A textbook of physiology. With C.S. Sherrington. Part 3. The central nervous system [electronic resource]. (London: Macmillan, 1897).

2. Hortsch, M. in The Sticky Synapse (eds. Umemori, H. \& Hortsch, M.) 1-9 (Springer New York, 2009).

3. Fairless, R. et al. Polarized targeting of neurexins to synapses is regulated by their C-terminal sequences. J. Neurosci. Off. J. Soc. Neurosci. 28, 1296912981 (2008).

4. Waldeyer-Hartz, W. von. Ueber einige neuere Forschungen im Gebiete der Anatomie des Centralnervensystems. (G. Tieme, 1891).

5. Berlucchi, G. Some aspects of the history of the law of dynamic polarization of the neuron. From William James to Sherrington, from Cajal and van Gehuchten to Golgi. J. Hist. Neurosci. 8, 191-201 (1999).

6. Robertson, J. D. Ultrastructure of two invertebrate synapses. Proc. Soc. Exp. Biol. Med. Soc. Exp. Biol. Med. N. Y. N 82, 219-223 (1953).

7. Katz, B. Quantal mechanism of neural transmitter release. Science 173, 123126 (1971).

8. Couteaux, R. \& Pécot-Dechavassine, M. [Synaptic vesicles and pouches at the level of 'active zones' of the neuromuscular junction]. Comptes Rendus Hebd. Séances Académie Sci. Sér. Sci. Nat. 271, 2346-2349 (1970). 
9. Dresbach, T., Qualmann, B., Kessels, M. M., Garner, C. C. \& Gundelfinger*, E. D. The presynaptic cytomatrix of brain synapses. Cell. Mol. Life Sci. CMLS 58, 94-116 (2001).

10. Zhai, R. G. \& Bellen, H. J. The architecture of the active zone in the presynaptic nerve terminal. Physiol. Bethesda Md 19, 262-270 (2004).

11. Gundelfinger, E. D. \& Fejtova, A. Molecular organization and plasticity of the cytomatrix at the active zone. Curr. Opin. Neurobiol. 22, 423-430 (2012).

12. Haucke, V., Neher, E. \& Sigrist, S. J. Protein scaffolds in the coupling of synaptic exocytosis and endocytosis. Nat. Rev. Neurosci. 12, 127-138 (2011).

13. Ackermann, F., Waites, C. L. \& Garner, C. C. Presynaptic active zones in invertebrates and vertebrates. EMBO Rep. 16, 923-938 (2015).

14. Jin, Y. \& Garner, C. C. Molecular mechanisms of presynaptic differentiation. Annu. Rev. Cell Dev. Biol. 24, 237-262 (2008).

15. Bruckner, J. J. et al. Fife, a Drosophila Piccolo-RIM Homolog, Promotes Active Zone Organization and Neurotransmitter Release. J. Neurosci. 32, 17048-17058 (2012).

16. Aravamudan, B., Fergestad, T., Davis, W. S., Rodesch, C. K. \& Broadie, K. Drosophila UNC-13 is essential for synaptic transmission. Nat. Neurosci. 2, 965-971 (1999).

17. Wang, Y. \& Südhof, T. C. Genomic definition of RIM proteins: evolutionary amplification of a family of synaptic regulatory proteins. Genomics $\mathbf{8 1}, 126-$ 137 (2003). 
18. Wagh, D. A. et al. Bruchpilot, a protein with homology to ELKS/CAST, is required for structural integrity and function of synaptic active zones in Drosophila. Neuron 49, 833-844 (2006).

19. Dai, Y. et al. SYD-2 Liprin-alpha organizes presynaptic active zone formation through ELKS. Nat. Neurosci. 9, 1479-1487 (2006).

20. Stigloher, C., Zhan, H., Zhen, M., Richmond, J. \& Bessereau, J.-L. The presynaptic dense projection of the Caenorhabditis elegans cholinergic neuromuscular junction localizes synaptic vesicles at the active zone through SYD-2/liprin and UNC-10/RIM-dependent interactions. J. Neurosci. Off. J. Soc. Neurosci. 31, 4388-4396 (2011).

21. Weimer, R. M. et al. UNC-13 and UNC-10/rim localize synaptic vesicles to specific membrane domains. J. Neurosci. Off. J. Soc. Neurosci. 26, 80408047 (2006).

22. Dieck, S. et al. Bassoon, a Novel Zinc-finger CAG/Glutamine-repeat Protein Selectively Localized at the Active Zone of Presynaptic Nerve Terminals. J. Cell Biol. 142, 499-509 (1998).

23. Altrock, W. D. et al. Functional inactivation of a fraction of excitatory synapses in mice deficient for the active zone protein bassoon. Neuron $37,787-800$ (2003).

24. Quiroz, F. G. \& Chilkoti, A. Sequence heuristics to encode phase behaviour in intrinsically disordered protein polymers. Nat. Mater. 14, 1164-1171 (2015).

25. Waites, C. L. et al. Bassoon and Piccolo maintain synapse integrity by regulating protein ubiquitination and degradation. EMBO J. 32, 954-969 (2013). 
26. tom Dieck, S. Molecular dissection of the photoreceptor ribbon synapse: physical interaction of Bassoon and RIBEYE is essential for the assembly of the ribbon complex. J. Cell Biol. 168, 825-836 (2005).

27. Hübler, D. et al. Differential spatial expression and subcellular localization of CtBP family members in rodent brain. PloS One 7, e39710 (2012).

28. Ivanova, D. et al. Synaptic activity controls localization and function of CtBP1 via binding to Bassoon and Piccolo. EMBO J. 34, 1056-1077 (2015).

29. Fejtova, A. et al. Dynein light chain regulates axonal trafficking and synaptic levels of Bassoon. J. Cell Biol. 185, 341-355 (2009).

30. Dresbach, T. Assembly of Active Zone Precursor Vesicles: OBLIGATORY TRAFFICKING OF PRESYNAPTIC CYTOMATRIX PROTEINS BASSOON AND PICCOLO VIA A TRANS-GOLGI COMPARTMENT. J. Biol. Chem. 281, 6038-6047 (2005).

31. Wang, X. et al. A protein interaction node at the neurotransmitter release site: domains of Aczonin/Piccolo, Bassoon, CAST, and rim converge on the $\mathrm{N}$ terminal domain of Munc13-1. J. Neurosci. Off. J. Soc. Neurosci. 29, 1258412596 (2009).

32. Maas, C. et al. Formation of Golgi-derived active zone precursor vesicles. J. Neurosci. Off. J. Soc. Neurosci. 32, 11095-11108 (2012).

33. Ohtsuka, T. et al. Cast: a novel protein of the cytomatrix at the active zone of synapses that forms a ternary complex with RIM1 and munc13-1. J. Cell Biol. 158, 577-590 (2002).

34. Davydova, D. et al. Bassoon Specifically Controls Presynaptic P/Q-type Ca2+ Channels via RIM-Binding Protein. Neuron 82, 181-194 (2014). 
35. Chen, J., Billings, S. E. \& Nishimune, H. Calcium channels link the musclederived synapse organizer laminin $\beta 2$ to Bassoon and CAST/Erc2 to organize presynaptic active zones. J. Neurosci. Off. J. Soc. Neurosci. 31, 512-525 (2011).

36. Popiolek, M. et al. D-amino acid oxidase activity is inhibited by an interaction with bassoon protein at the presynaptic active zone. J. Biol. Chem. 286, 28867-28875 (2011).

37. Kremer, T. et al. Mover is a novel vertebrate-specific presynaptic protein with differential distribution at subsets of CNS synapses. FEBS Lett. 581, 4727$4733(2007)$.

38. Ahmed, S. et al. Mover is a homomeric phospho-protein present on synaptic vesicles. PloS One 8, e63474 (2013).

39. Körber, C. et al. Modulation of Presynaptic Release Probability by the Vertebrate-Specific Protein Mover. Neuron 87, 521-533 (2015).

40. Gundelfinger, E. D., Reissner, C. \& Garner, C. C. Role of Bassoon and Piccolo in Assembly and Molecular Organization of the Active Zone. Front. Synaptic Neurosci. 7, (2016).

41. Mukherjee, K. et al. Piccolo and bassoon maintain synaptic vesicle clustering without directly participating in vesicle exocytosis. Proc. Natl. Acad. Sci. U. S. A. 107, 6504-6509 (2010).

42. Hallermann, S. et al. Bassoon Speeds Vesicle Reloading at a Central Excitatory Synapse. Neuron 68, 710-723 (2010).

43. Dick, O. et al. The presynaptic active zone protein bassoon is essential for photoreceptor ribbon synapse formation in the retina. Neuron $\mathbf{3 7}, \mathbf{7 7 5 - 7 8 6}$ (2003). 
44. Khimich, D. et al. Hair cell synaptic ribbons are essential for synchronous auditory signalling. Nature 434, 889-894 (2005).

45. Hallermann, S. \& Silver, R. A. Sustaining rapid vesicular release at active zones: potential roles for vesicle tethering. Trends Neurosci. 36, 185-194 (2013).

46. Mendoza Schulz, A. The role of the presynaptic scaffold protein Bassoon in synaptic transmission at the mouse endbulb of Held synapse. (2013).

47. Schröder, M. S. et al. Regulation of Presynaptic Anchoring of the Scaffold Protein Bassoon by Phosphorylation-Dependent Interaction with 14-3-3 Adaptor Proteins. PLoS ONE 8, (2013).

48. Takao-Rikitsu, E. et al. Physical and functional interaction of the active zone proteins, CAST, RIM1, and Bassoon, in neurotransmitter release. J. Cell Biol. 164, 301-311 (2004).

49. Fenster, S. D. et al. Piccolo, a presynaptic zinc finger protein structurally related to bassoon. Neuron 25, 203-214 (2000).

50. Fenster, S. D. et al. Interactions between Piccolo and the actin/dynaminbinding protein Abp1 link vesicle endocytosis to presynaptic active zones. J. Biol. Chem. 278, 20268-20277 (2003).

51. Kessels, M. M., Engqvist-Goldstein, A. E., Drubin, D. G. \& Qualmann, B. Mammalian Abp1, a signal-responsive F-actin-binding protein, links the actin cytoskeleton to endocytosis via the GTPase dynamin. J. Cell Biol. 153, 351366 (2001).

52. Wang, X. et al. Aczonin, a 550-Kd Putative Scaffolding Protein of Presynaptic Active Zones, Shares Homology Regions with Rim and Bassoon and Binds Profilin. J. Cell Biol. 147, 151-162 (1999). 
53. Gerber, S. H., Garcia, J., Rizo, J. \& Südhof, T. C. An unusual C(2)-domain in the active-zone protein piccolo: implications for $\mathrm{Ca}(2+)$ regulation of neurotransmitter release. EMBO J. 20, 1605-1619 (2001).

54. Wang, Y., Okamoto, M., Schmitz, F., Hofmann, K. \& Südhof, T. C. Rim is a putative Rab3 effector in regulating synaptic-vesicle fusion. Nature $\mathbf{3 8 8 , 5 9 3 -}$ 598 (1997).

55. Betz, A. et al. Functional interaction of the active zone proteins Munc13-1 and RIM1 in synaptic vesicle priming. Neuron 30, 183-196 (2001).

56. Maruyama, I. N. \& Brenner, S. A phorbol ester/diacylglycerol-binding protein encoded by the unc-13 gene of Caenorhabditis elegans. Proc. Natl. Acad. Sci. U. S. A. 88, 5729-5733 (1991).

57. Fujimoto, K. et al. Piccolo, a Ca2+ sensor in pancreatic beta-cells. Involvement of cAMP-GEFII.Rim2. Piccolo complex in cAMP-dependent exocytosis. J. Biol. Chem. 277, 50497-50502 (2002).

58. Brose, N., Hofmann, K., Hata, Y. \& Südhof, T. C. Mammalian homologues of Caenorhabditis elegans unc-13 gene define novel family of C2-domain proteins. J. Biol. Chem. 270, 25273-25280 (1995).

59. Basu, J. et al. A minimal domain responsible for Munc13 activity. Nat. Struct. Mol. Biol. 12, 1017-1018 (2005).

60. Varoqueaux, F. et al. Total arrest of spontaneous and evoked synaptic transmission but normal synaptogenesis in the absence of Munc13-mediated vesicle priming. Proc. Natl. Acad. Sci. U. S. A. 99, 9037-9042 (2002).

61. Augustin, I., Rosenmund, C., Südhof, T. C. \& Brose, N. Munc13-1 is essential for fusion competence of glutamatergic synaptic vesicles. Nature $400,457-$ 461 (1999). 
62. Lu, H.-C. et al. Role of efficient neurotransmitter release in barrel map development. J. Neurosci. Off. J. Soc. Neurosci. 26, 2692-2703 (2006).

63. Kaeser, P. S. Pushing synaptic vesicles over the RIM. Cell. Logist. 1, 106110 (2011).

64. Wang, Y., Liu, X., Biederer, T. \& Südhof, T. C. A family of RIM-binding proteins regulated by alternative splicing: Implications for the genesis of synaptic active zones. Proc. Natl. Acad. Sci. 99, 14464-14469 (2002).

65. Monier, S., Jollivet, F., Janoueix-Lerosey, I., Johannes, L. \& Goud, B. Characterization of novel Rab6-interacting proteins involved in endosome-toTGN transport. Traffic Cph. Den. 3, 289-297 (2002).

66. tom Dieck, S. et al. Deletion of the presynaptic scaffold CAST reduces active zone size in rod photoreceptors and impairs visual processing. J. Neurosci. Off. J. Soc. Neurosci. 32, 12192-12203 (2012).

67. Kaeser, P. S. et al. ELKS2a/CAST Deletion Selectively Increases Neurotransmitter Release at Inhibitory Synapses. Neuron 64, 227-239 (2009).

68. Zhai, R. G. et al. Assembling the presynaptic active zone: a characterization of an active one precursor vesicle. Neuron 29, 131-143 (2001).

69. Shapira, M. et al. Unitary assembly of presynaptic active zones from PiccoloBassoon transport vesicles. Neuron 38, 237-252 (2003).

70. Dresbach, T. et al. Functional regions of the presynaptic cytomatrix protein bassoon: significance for synaptic targeting and cytomatrix anchoring. Mol. Cell. Neurosci. 23, 279-291 (2003). 
71. Tao-Cheng, J.-H. Ultrastructural localization of active zone and synaptic vesicle proteins in a preassembled multi-vesicle transport aggregate. Neuroscience 150, 575-584 (2007).

72. Cai, Q., Pan, P.-Y. \& Sheng, Z.-H. Syntabulin-kinesin-1 family member 5Bmediated axonal transport contributes to activity-dependent presynaptic assembly. J. Neurosci. Off. J. Soc. Neurosci. 27, 7284-7296 (2007).

73. Friedman, H. V., Bresler, T., Garner, C. C. \& Ziv, N. E. Assembly of new individual excitatory synapses: time course and temporal order of synaptic molecule recruitment. Neuron 27, 57-69 (2000).

74. Mochida, S. Presynaptic Terminals. (Springer, 2015).

75. Siddiqui, T. J. \& Craig, A. M. Synaptic organizing complexes. Curr. Opin. Neurobiol. 21, 132-143 (2011).

76. Dani, A., Huang, B., Bergan, J., Dulac, C. \& Zhuang, X. Super-resolution Imaging of Chemical Synapses in the Brain. Neuron 68, 843-856 (2010).

77. Limbach, C. et al. Molecular in situ topology of Aczonin/Piccolo and associated proteins at the mammalian neurotransmitter release site. Proc. Natl. Acad. Sci. 108, E392-E401 (2011).

78. Harke, B. et al. Resolution scaling in STED microscopy. Opt. Express 16, 4154-4162 (2008).

79. Göttfert, F. et al. Coaligned Dual-Channel STED Nanoscopy and Molecular Diffusion Analysis at $20 \mathrm{~nm}$ Resolution. Biophys. J. 105, L01-L03 (2013).

80. Opazo, F. et al. Aptamers as potential tools for super-resolution microscopy. Nat. Methods 9, 938-939 (2012). 
81. Naturally occurring antibodies devoid of light chains. Available at: http://www.nature.com/nature/journal/v363/n6428/abs/363446a0.html. (Accessed: 11th February 2016)

82. Ries, J., Kaplan, C., Platonova, E., Eghlidi, H. \& Ewers, H. A simple, versatile method for GFP-based super-resolution microscopy via nanobodies. Nat. Methods 9, 582-584 (2012).

83. Platonova, E. et al. Single-molecule microscopy of molecules tagged with GFP or RFP derivatives in mammalian cells using nanobody binders. Methods 88, 89-97 (2015).

84. Trinkle-Mulcahy, L. et al. Identifying specific protein interaction partners using quantitative mass spectrometry and bead proteomes. J. Cell Biol. 183, 223239 (2008).

85. Rust, M. J., Bates, M. \& Zhuang, X. Sub-diffraction-limit imaging by stochastic optical reconstruction microscopy (STORM). Nat. Methods 3, 793-796 (2006).

86. Mielczarek, E. V., Knox, R. S. \& Greenbaum, E. Biological Physics. (Springer Science \& Business Media, 1993).

87. Shcherbo, D. et al. Practical and reliable FRET/FLIM pair of fluorescent proteins. BMC Biotechnol. 9, 24 (2009).

88. Bückers, J., Wildanger, D., Vicidomini, G., Kastrup, L. \& Hell, S. W. Simultaneous multi-lifetime multi-color STED imaging for colocalization analyses. Opt. Express 19, 3130-3143 (2011).

89. van den Bogaart, G. et al. One SNARE complex is sufficient for membrane fusion. Nat. Struct. 38 Mol. Biol. 17, 358-364 (2010).

90. Banker, G. \& Goslin, K. Culturing Nerve Cells. (1998). 
91. Schonle, A. Imspector Image Acquisition \& Analysis Software, v0.10. (2006).

92. Costes, S. V. et al. Automatic and Quantitative Measurement of ProteinProtein Colocalization in Live Cells. Biophys. J. 86, 3993-4003 (2004).

93. Ziv, N. E. \& Garner, C. C. Cellular and molecular mechanisms of presynaptic assembly. Nat. Rev. Neurosci. 5, 385-399 (2004).

94. Wildanger, D., Medda, R., Kastrup, L. \& Hell, S. W. A compact STED microscope providing 3D nanoscale resolution. J. Microsc. 236, 35-43 (2009).

95. Ma, H., Cai, Q., Lu, W., Sheng, Z.-H. \& Mochida, S. KIF5B Motor Adaptor Syntabulin Maintains Synaptic Transmission in Sympathetic Neurons. J. Neurosci. 29, 13019-13029 (2009).

96. Ahmari, S. E., Buchanan, J. \& Smith, S. J. Assembly of presynaptic active zones from cytoplasmic transport packets. Nat. Neurosci. 3, 445-451 (2000).

97. Dean, C. et al. Distinct subsets of Syt-IV/BDNF vesicles are sorted to axons versus dendrites and recruited to synapses by activity. J. Neurosci. Off. J. Soc. Neurosci. 32, 5398-5413 (2012).

98. Zhang, Z. et al. Release mode of large and small dense-core vesicles specified by different synaptotagmin isoforms in PC12 cells. Mol. Biol. Cell 22, 2324-2336 (2011).

99. Tsuriel, S. et al. Exchange and Redistribution Dynamics of the Cytoskeleton of the Active Zone Molecule Bassoon. J. Neurosci. 29, 351-358 (2009).

100. Betz, A. et al. Munc13-1 Is a Presynaptic Phorbol Ester Receptor that Enhances Neurotransmitter Release. Neuron 21, 123-136 (1998).

101. Stevens, D. R. et al. Identification of the Minimal Protein Domain Required for Priming Activity of Munc13-1. Curr. Biol. 15, 2243-2248 (2005). 
102. Spiwoks-Becker, I., Lamberti, R., tom Dieck, S. \& Spessert, R. Evidence for synergistic and complementary roles of Bassoon and darkness in organizing the ribbon synapse. Neuroscience 236, 149-159 (2013).

103. Spilker, C., Dresbach, T. \& Braunewell, K.-H. Reversible Translocation and Activity-Dependent Localization of the Calcium-Myristoyl Switch Protein VILIP-1 to Different Membrane Compartments in Living Hippocampal Neurons. J. Neurosci. 22, 7331-7339 (2002).

104. Qasba, P. K., Ramakrishnan, B. \& Boeggeman, E. Structure and Function

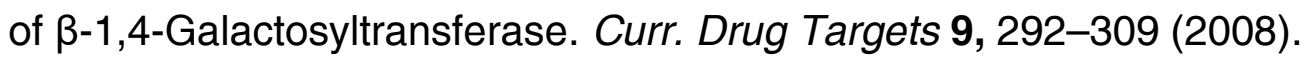

105. Bos, K., Wraight, C. \& Stanley, K. K. TGN38 is maintained in the transGolgi network by a tyrosine-containing motif in the cytoplasmic domain. EMBO J. 12, 2219-2228 (1993).

106. Gu, F., Crump, C. M. \& Thomas, G. Trans-Golgi network sorting. Cell. Mol. Life Sci. CMLS 58, 1067-1084 (2001).

107. Bock, J. B., Klumperman, J., Davanger, S. \& Scheller, R. H. Syntaxin 6 functions in trans-Golgi network vesicle trafficking. Mol. Biol. Cell 8, 12611271 (1997).

108. Jung, J.-J., Inamdar, S. M., Tiwari, A. \& Choudhury, A. Regulation of intracellular membrane trafficking and cell dynamics by syntaxin-6. Biosci. Rep. 32, 383-391 (2012).

109. Ribrault, C., Sekimoto, K. \& Triller, A. From the stochasticity of molecular processes to the variability of synaptic transmission. Nat. Rev. Neurosci. 12, 375-387 (2011).

110. Garner, C. C., Kindler, S. \& Gundelfinger, E. D. Molecular determinants of presynaptic active zones. Curr. Opin. Neurobiol. 10, 321-327 (2000). 
111. Ko, J., Na, M., Kim, S., Lee, J.-R. \& Kim, E. Interaction of the ERC Family of RIM-binding Proteins with the Liprin- $\alpha$ Family of Multidomain Proteins. J. Biol. Chem. 278, 42377-42385 (2003).

112. Régnier-Vigouroux, A., Tooze, S. A. \& Huttner, W. B. Newly synthesized synaptophysin is transported to synaptic-like microvesicles via constitutive secretory vesicles and the plasma membrane. EMBO J. 10, 3589-3601 (1991).

113. Matteoli, M., Coco, S., Schenk, U. \& Verderio, C. Vesicle turnover in developing neurons: how to build a presynaptic terminal. Trends Cell Biol. 14, 133-140 (2004).

114. Park, J. J., Gondré-Lewis, M. C., Eiden, L. E. \& Loh, Y. P. A distinct transGolgi network subcompartment for sorting of synaptic and granule proteins in neurons and neuroendocrine cells. J. Cell Sci. 124, 735-744 (2011).

115. Kneussel, M. Dynamic regulation of $\operatorname{GABA}(\mathrm{A})$ receptors at synaptic sites. Brain Res. Brain Res. Rev. 39, 74-83 (2002).

116. Sytnyk, V., Leshchyns'ka, I., Dityatev, A. \& Schachner, M. Trans-Golgi network delivery of synaptic proteins in synaptogenesis. J. Cell Sci. 117, 381388 (2004).

117. Sytnyk, V. et al. Neural cell adhesion molecule promotes accumulation of TGN organelles at sites of neuron-to-neuron contacts. J. Cell Biol. 159, 649661 (2002).

118. Nakata, T., Terada, S. \& Hirokawa, N. Visualization of the dynamics of synaptic vesicle and plasma membrane proteins in living axons. J. Cell Biol. 140, 659-674 (1998). 
119. Alberts, B., Johnson, A. \& Lewis, J. in Molecular Biology of the Cell (Garland Science, 2002).

120. Jeyifous, O. et al. SAP97 and CASK mediate sorting of NMDA receptors through a previously unknown secretory pathway. Nat. Neurosci. 12, 10111019 (2009).

121. Pfeffer, S. Membrane Domains in the Secretory and Endocytic Pathways. Cell 112, 507-517 (2003).

122. Ladinsky, M. S., Kremer, J. R., Furcinitti, P. S., McIntosh, J. R. \& Howell, K. E. HVEM tomography of the trans-Golgi network: structural insights and identification of a lace-like vesicle coat. J. Cell Biol. 127, 29-38 (1994).

123. Tooze, J. \& Tooze, S. A. Clathrin-coated vesicular transport of secretory proteins during the formation of $\mathrm{ACTH}$-containing secretory granules in AtT20 cells. J. Cell Biol. 103, 839-850 (1986).

124. Orci, L. et al. Direct identification of prohormone conversion site in insulinsecreting cells. Cell 42, 671-681 (1985).

125. Dikeakos, J. D. \& Reudelhuber, T. L. Sending proteins to dense core secretory granules: still a lot to sort out. J. Cell Biol. 177, 191-196 (2007).

126. Dhanvantari, S. \& Loh, Y. P. Lipid raft association of carboxypeptidase E is necessary for its function as a regulated secretory pathway sorting receptor. J. Biol. Chem. 275, 29887-29893 (2000).

127. Mikhaylova, M. et al. Calneurons provide a calcium threshold for transGolgi network to plasma membrane trafficking. Proc. Natl. Acad. Sci. 106, 9093-9098 (2009).

128. Bonazzi, M. et al. CtBP3/BARS drives membrane fission in dynaminindependent transport pathways. Nat. Cell Biol. 7, 570-580 (2005). 
129. Chavrier, P. \& Goud, B. The role of ARF and Rab GTPases in membrane transport. Curr. Opin. Cell Biol. 11, 466-475 (1999).

130. Ko, J. et al. Interaction between Liprin- $\alpha$ and GIT1 Is Required for AMPA Receptor Targeting. J. Neurosci. 23, 1667-1677 (2003).

131. Bury, L. A. \& Sabo, S. L. Coordinated trafficking of synaptic vesicle and active zone proteins prior to synapse formation. Neural Develop. 6, 24 (2011).

132. Siebert, M. et al. A high affinity RIM-binding protein/Aplip1 interaction prevents the formation of ectopic axonal active zones. eLife 4, e06935 (2015).

133. Bresler, T. et al. The dynamics of SAP90/PSD-95 recruitment to new synaptic junctions. Mol. Cell. Neurosci. 18, 149-167 (2001).

134. Prange, O. \& Murphy, T. H. Modular transport of postsynaptic density-95 clusters and association with stable spine precursors during early development of cortical neurons. J. Neurosci. Off. J. Soc. Neurosci. 21, 9325$9333(2001)$.

135. Wong, M. Y. et al. Neuropeptide Delivery to Synapses by Long-Range Vesicle Circulation and Sporadic Capture. Cell 148, 1029-1038 (2012).

136. Patel, M. R. et al. Hierarchical assembly of presynaptic components in defined C. elegans synapses. Nat. Neurosci. 9, 1488-1498 (2006).

137. Fouquet, W. et al. Maturation of active zone assembly by Drosophila Bruchpilot. J. Cell Biol. 186, 129-145 (2009).

138. Waites, C. L. \& Garner, C. C. Presynaptic function in health and disease. Trends Neurosci. 34, 326-337 (2011).

139. Rimsa, V., Eadsforth, T. C. \& Hunter, W. N. Two high-resolution structures of the human E3 ubiquitin ligase Siah1. Acta Crystallogr. Sect. F 69, 13391343 (2013). 


\section{Appendix}

Appendix A: The sequence of double-tagged full-length Bassoon construct:RFP-tag GFP-tag

GCTCCTGGCCGAGAGAGCCCCCGAGAGACAAGGGCCCAAGGCCTGTCAGGCCAGGAGGCCGAAGGTCCCCGCAGGACACTGCAGGTAGACAGCAGGACACAGAGATCAGGACGGTCCCCCTCCGTGTCACCAGACAGAGGCAGCACTCCCA
CATCACCCTATTCCGTCCCCCAGATTGCCCCTCTTCCCAGCAGCACCCTGTGTCCAATATGCAAGACATCGGACCTCACATCAACCTCCAGCCAGCCAAACTTCAACACCTGCACCCAGTGTCACAACAAGGTCTGCAACCAATGTGGGTTCAAC CCCAACCTCACCTCACCAGGTGAAGGAGTGGCTCTGTTTGAACTGTCAGATGCAGAGGGCCCTGGGAATGGACATGACCACTGCACCTCGGTCCAAGAGCCAGCAGCAGCTACACTCCCCGGCCCTGTCTCCTGCCCACTCCCCAGCCAAA CCCCTCTCTCTAACAAGCCTTCAACAGCTGAGCCCAGGCCACCTGCAGGAGAGGCCCAGGGCAAAAGGGCCACCACAGTGCCCTCCGGGCTTGGTGCTGCTGAACAGACCCAGGAGGGGCTCACCGGGAAGCTCTTTGGCCTTGGAGCATCA GCCAACCCCTGGAGCCAAAACGGAGCCTGGGCCTAGAACAGGTCCTGGATCAGGGCCTGGGGCCCTGGCAAAAACTGGAGGAACCCCCAGTCCAAAGCATGGCAGAGCAGACCATCAGGCAGCATCCAAAGCTGCTGCCAAGCCAAAGACCA

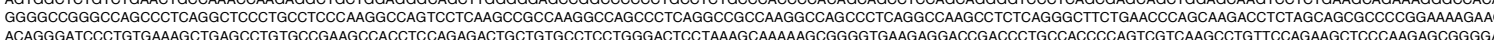
GGCTGAGGAACCTGTCCCCAAGCCTTACTCTCAGGATCTGTCTCGAAGCCCACAAAGCCTCAGTGATACAGGCTACTCCTCCGATGGCGTCTCCAGCTCCCAGAGTGAGATCACAGGTGTTGTACAGCAAGAAGTGGAGCAACTTGACAGTGCG

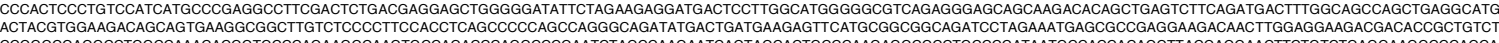
GAGCCCACTGACGGAGCCCTTAGGGAGGTCTCCGACGCTTTAAAACTATTGAGCTCAACAGCACAGGTAGCTATGGTCACGAGCTAGACCTGGGCCAAGGCTCTGATCCCAACCTAGACCGGGGCCTGAGCTGGAGATGGAGAGCCTTACA GGCTCGCCTGAAGACCGCTCTCGTGGGGAGCACTCCTCTACACTGCCGGCTTCCACGCCTAGCTATACATCTGGCACCTCACCCACCTCCCTGTCTTCCCTGGAGGAGGACAGTGACAGCAGCCCCAGCCGCAGGCAGCGGCTAGAAGAAGC CACAGCCCGCAAGACCCGGGGTGACAAGGAAGAACTTCGGGCTCAGCGGCGGGGTGAGCGCTCCAAGACACCGCCTAGTAACCTGTCACCCATCGAAGATGCGTCCCCTACAGAGGAGCTGAGGCAGGCAGCGGAGATGGAGGAGCTACAC GTTCCACCACGCCCAGTTCTGGACGGCCCCTTAAGAGTGCGGAGGAGGCCTACGAGGACATGATGAGAAAAGCTGAGCTGCTCCAGAGACAGCAGGGCCAGGCGGCAGGGGCCCGGGGACCCCATGGCGGCCCCTCTCAGCCCACAGGCCC
CCGCAGCCAGGGCTCCTTTAATACCAAGACACCCAAGACCATGACTATGGCGGCAGGGCTTCTCAGCCTGCGGCAGATGGCACGCCCGCAGGCCTCGGAGCAACAGTGTATGAGGAGATCCTTCAGACATCCCAGAGCATAGCCCGGATGC GGCAGGCCTCCTCGCGGGATCTGGCCTTCACTGAGGACAAAAAGAAGGAGAAGCAGTTTCTGAATGCGGAGAGTGCATACATGGACCCAATGAAGCAAAATGGTGGCCCACTTACCCCTGGGACTAGCCCCACCCAACTTGCTGCTCCTGTGTC TCTCCCAGGGTCCTGGAACTCCAGCCACCACAGCCATGGCGCCTTGTCCAGCCAGCCTTCCACGGGGGTATATGACTCCAGCCGGCCCTGAGCGCAGCCCATCAACATCTTCCACAATACACAGCTATGGACAGCCCCCAACCACTGCTAACTA TGGGTCTCAGACGGAGGAGCTGCCTCACGCTCCCAGTGGCCCTGGTGGAAGTGGGCGGGCCTCCAGAGAGAAGCCTCTGAGTGGGGGTGATGGTGAGGTTGGTCCCCCCCAGCCTTCTCGGGGATATTCTTATTTTACGGGCTCTAGCCCAC AGGCACGCAGACGCCACACCGACCTAGCACTCCTCGCTTGGTGTGGCAGCAGTCTTCCCAGGAGGCTCCTGTTATGGTCATCACGCTAGCTTCAGATGCTTCTAGCCAGACCCGAATGGTACACGCCAGTGCCTCTACTTCCCCTCTGTGCTCG CTCCCTGCTGAAAACATCTCCTTATGCCGGATCTCCTCTGTCCCTGGAACATCTAGAGTTGAGCCGGGCCCCAGGCCCCCGGGCACCGCAGTGGTCGACCTTCGCACGGCTGTCAAGCCCACACCCATTATCCTTACCGACCAGGGCATGGATC CATGGCCTCATCTGTGCTCATGGCGCAGCAAAAGCAACCAGTGGTATATGGAGATCCTTTCAGAGCCGCCTCGACTTTGGCCAGGGTTCAGGTAGCCCTGTGTGCCTGGCCCAGGTCAAGCAAGTAGAGCAGGCTGTCCAGACAGCCCCATAC GGTGCTGAACCCCACCGGGCTACTCCTGCAGAGCTCCGGGCACATGCTCTACCAGGTACCAGGAAGCCACACACAGTGGTGGTGCAGATGGGAGAGGGCGCAGCCGGCACGGTGACTACCCTGCTCCCAGAAGAGCCAGCAGGAGCCCTGG CCCCTTCTACAGTCCCAGAGACCCTGAGCCTCCTGAGCCCCTCACCTTCCGGGCACAAGGGGTCGTAGGACCTGGGCCCCATGAAGAACAGAGGCCCTACCCACAGGGCTTGCCCGGTAGGCTCTACTCCTCCATGTCTGACACCAATTTGGC TGAAGCTGGTCTCAACTACCACGCCCAGAGGATTGGGCAGCTCTTCCAGGGCCCTGGGAGGGACTCAGCTGTGGATCTCAGCTCGCTGAAGCATTCTTACAGCTTAGGCTTCGCTGATGGACGCTACTTGGGGCAGGGCTTACAGTATGGCTCG TTCACGGACCTGCGCCACCCCACAGACCTTTTGTCTCACCCACTGCCCATGAGGCGCTACAGCTCTGTGTCAAACATCTATTCGGATCACCGGTACGGCCCACGGGGAGATGCCGTCGGCTTTCAGGAGGCCAGCCTGGCCCAGTACAGTGCC

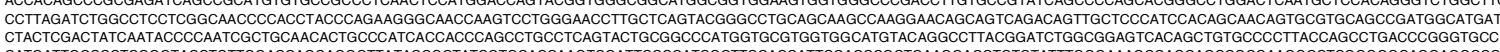
GCCAAGGCCTGAGCTACCAGCAGGGGGTGCTCGAGAAGAACCTCTCTCCACAACTGCCCCTCCTGCTGTCATCAAGGAAGCCCCGGTGGCCCAGGCCCCGGCCCCACCACCAGGCCAAAAGCCAGCAGGAGATGCTGCTGCTGGGAGTGGC AGTGGAGTCCTCGGCCGGCCTGTGATGGAGAAGGAGGAGGCATCTCAGGAGGACCGGCAGCGAAAGCAACAGGAGCAGCTGCTGCAGCTGGAGCGGGAACGGGTAGAGTTGGAAAAGCTCCGGCAGTTGCGGCTACAGGAGGAGCTGGAG

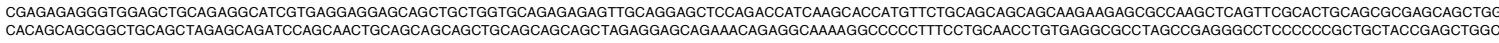

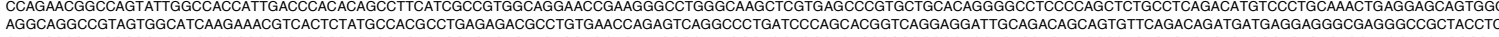
CTGACCCGTAGGCGCAGGACACGGCGCAGTGCCGACTGCAGTGTGCAGACAGATGACGAGGACAATGCCGAATGGGAGCAGCCTGTCCGCCGCCGCAGGTCTCGTCTTTCCCGTCACTCAGACTCAGGCTCTGACAGCAAGCACGAGGCCTC AGATCGTCAGATACATATCAGCACCAGAAAAGACTGGGGGAGGGGAGAGCCTGGCCTGCCAGACAGAACCCGATGGGCAGGCTCAGGGTGTGGCTGGGCCACAGCTCATAGGACCAACTGCCATCAGCCCCTACCTACCTGGCATCCAGATAG GTCCCCACACCGGCTCCTGGACACCTCGTTTGCTTCCAGTGAGAGGCTGAACAAGGCTCATGTGAGTCCCCAGAAGCAGTTCATAGCTGACAGCACTCTTCGCCAGCAGACTCTGCCGCGCCCCATGAAGACCCTGCAGCGGTCCCTGTCCGA CCCTAAGCCCCTGAGCCCCACCGCCGAGGAGTCTGCCAAAGAGAGATTCTCCCTCTACCAGCACCAGGGGGGACTAGGTAGCCAGGTGTCGGCGCTGCCACCCAACGGCCTGGTCCGCAAGGTGAAGCGGACACTGCCCAGCCCCCCTCCA ATGAATCCACCAAGCTGCGCAAGAAGCAGGCGGAGTTGGACGAGGAGGAGAAGGAGATTGATGCCAAGCTCAAGTACCTGGAGCTGGGCATCACCCAGCGCAAAGAGTCTTTGGCCAAAGACCGGGTTGGCCGTGACTACCCACCTTTACGT GCCAAGCAACCGGCTTTCACCCCACAGGCCATTACGCAGCCCCAACGCCCATGCCAACCACGCAGAGCGCCCCTTTTCCAGTCCAAGCTGACAGTCACGCTGCCCACCAGAAGCCGCGCCAGACCTCACTGGCTGATTTGGAGCAGAAGG

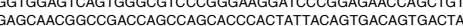
CCGGAAGCAAGGATGGAGCAAAAGATCTCCAAGTTCTCACCTATCGAGGAGGCCAGGACGTGGAATCAGACCTGGCCTCCTATCCCCCACCCACGGTCAGCAGCAGCCTGACCTCTCGGAGCAGAAAGTTCCAAGACGAAATCACCTATGG GCTCAAGAAGAATGTGTATGAACAGCAGAGG TACTACGGGGTGTCCAGCCGGGACACAGCGGAGGAGGATGACGGCATGTATGGTGGTAGCAGCCGGTCCCGAGTGGCATGGGCATACAGTGGGGAGAAGCTGTCCAGCCATGATTTCAGCA GCCGAAGCAAAGGGTATGAACGGGAACGGGAGACTGCAGAGCGACTTCAAAAGGCGGGGCCCAAGCCCTCATCCCTGAGCATGGCTCATGGACGGGCACGGCCCCCCATGAGGAGCCAGGCCTCTGAAGAGGAGAGCCCTGTCAGCCCCT ACTCCCACTGCGTGGTTTGGACAGTGAAGCGTATCACCTGGGCCAGGAGGAGACAGACT GGTTTGATAAGCCCCGAGATGCCCGCTCCGACCGGTTCAGGCACCATGGGGGGCATACAGTCTCCTCCTCCCAGAAGCGAGGCCCTGCCAGG
CACAGCTACCATGACTACGATGAGCCCCCTGAGGAGGGCCTGTGGCCTCATGATGAGGGTGGTCCAGGCCGGCATACCTCAGCCAAGGAGCACCGGCACCACGGTGACCACGGGAGGCACTCAGGCCGCCATGCTGGTGAGGAGCCAGGGC GCCGTGCTGCCAGACCACATGCTCGGGACATGGGTCGCCACGAGACCCGGCCTCACCCTCAGGCCAGCCCTGCCCCTGCCATGCAAAAGAAGGGTCAGCCTGGGTACCCCAGCTCTGCTGATTACTCACAGCCATCCCGGGCTCCGTCAGCA TACCACCATGCTCATGACAGCAAAAAGGGCTCCCGGCAGGCCACACTCTGGGCCTACTGTACTGCAGCCAAAGCCAGAAGCCCAGGCACAGCCACAGATGCAGGGTCGGCAGGCGGTTCCGGGGACCGCAGCAATCACAGCCACCATCGGCCAG

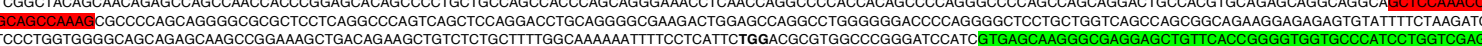
CTGGACGGCGACGTAAACGGCCACAAGTTAAGCGTGTCCGGCGAGGGCGAGGGCGATGCCACCTACGGCAAGCTGACCCTGAAGTTCATCTGCACCACCGGCAAGCTGCCCGTGCCCTGGCCCCACCCTCGTGACCACCCTGACCTACGGCG TCGAGGACGGCAGCGTGCAGCTCGCCGACCACTACCAGCAGAACACCCCCATCGGCGACGGCCCCGTGCTGCTGCCCGACAACCACTACCTGAGCACCCAGTCCAAGCTGAGCAAAGACCCCAACGAGAAGCGCGATCACATGGTCCTGCT

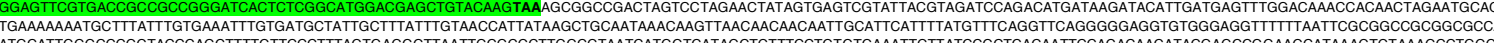
GTGCCTAATGAGTGAGCTAACTCACATTAATTGCGTTGCGCTCACTGCCCGCTTTCCAGTCGGGAAACCTGTCGTGCCAGCTGCATTAATGAATCGGCCAACGCGCGGGGAGAGGCGGTTGCGTATTGGGCGCTCTTCCGCTTCCTCGCTCAC GCTGGCGTTTTCCATAGGCTCCGCCCCCCTGACGAGCATCACAAAAATCGACGCTCAAGTCAGAGGTGGCGAAACCCGACAGGACTATAAAGATACCAGGCGTTTCCCCCTGGAAGCTCCCTCGTGCGCTCTCCTG TTCCGACCCTGCCGCT ACTATCGTCTTGAGTCCAACCCGGTAAGACACAACTATCGCCACTGGCAGCAGCCACTGGTAACAGGATTAGCAGAGCGAGGTATGTAGGCGGTGCTACAGAGTTCTTGAAGTGGTGGCCTAACTACGGCTACACTAGAAGGACAGTATTTGGT
ATCTGCGCTCTGCTGAAGCCAGTTACCTTCGGAAAAGAGTTGGTAGCTCTTGATCCGGCAAACAAACACCGCTGGTAGCGGTGGTTTTTTGTTTGCAAGCAGCAGATTACGCGCAGAAAAAAGGATTCAAGAAGATCCTTGGATCTTTCTA TGGGGTCTGACGCTCAGTGGACGAAACTCACGTTAAGGGATTTTGGTCATGAGATTATCAAAAAGGATCTTCACCTAGATCCTTTTAAATTAAAAATGAAGTTTTAAATCAATCTAAAGTATATATGAGTAAACTTGGTCTGACAGTTACCAATGC TTATCAGCAATAAACCAGCCAGCCGGAAGGGCCGAGCGCAGAAGTGGTCCTGCAACTTTATCCGCCTCCATCCAGTCTATTAATTGTTGCCGGGAAGCTAGAGTAAGTAGTTCGCCAGTTAATAGTTTGCGCAAGGTTGTTGCCATTGCTACAGG ACTCATGGTTATGGCAGCACTGCATAATTCTCTTACTGTCATGCCATCCGTAAGATGCTTTTCTGTGACTGGTGAGTACTCAACCAAGTCATTCTGAGAATAGTGTATGCGGCGACGAGTTGCTCTTGCCCGGCGTCAATACGGGATAATACCGC
GCCACATAGCAGAACTTTAAAAGTGCTCATCATTGGAAACGTTCTTCGGGGCGAAAACTCTCAAGATCTTACCGCTGTTGAGATCCAGTTCGATGTAACCCACTGGTGCACCCAACTGATCTTCAGCATCTTTIACTTCACCAGCGTTCTGGG TGAGCAAAAACAGGAAGGCAAAATGCCGCAAAAAAGGGATAAGGGCGACACGGAATGTTGAATACTCATACTCTTCCTTTTTCAATATTATTGAAGCATTATCAGGGTATTGTCTCATGAGCGGATACATATTTGAATGTATTTAGAAAAATAA GAGATAGGGTTGAGTGTTGTTCCAGTTTGGAACAAGAGTCCACTATTAAAGAACGTGGACTCCAACGTCAAAGGGCGAAAAACCGTCTATCAGGGCGATGGCCCACTACGTGAACCATCACCCTAATCAAGTTTTTGGGGTCGAGGTGCCGTAA AGCACTAAATCGGAACCCTAAAGGGAGCCCCGATTTAGAGCTTGACGGGGAAGCCGGCGAAGGTGGGAGAAGGAAGGAAGAAAGGAAAGGAGGGGGGGCTAGGGCGCTGGCAAGTGTAGCGGTCACGCTGCGCGTAACCACCACA TATGGTGGATCTGGACCTGTGCCAATTCAATATGGCGTATATGGACTCGTGCCAATTCAATATGGTGGATCTGGACCCCAGCCAATTCAATATGGCGGACTTGGCACCATGCCAATTCAATATGGCGGACTTGGCACTGTGCCAACTGGGGAGGG CTATTGGCCAGGTTCAATACTATGTATTGGCCCTATGCCATATAGTATTCCATATATGGGTTTTCCTATTGACGTAGATAGCCCCTCCCAATGGGCGGTCCCATATACCATATATGGGGCTTCCTAATACCGCCCATAGCCACTCCCCCATTGACGT GATGGCTCATTGCCCATTCATATCCGTTCTCACGCCCCCTATTGACGTCAATGACGGTAAATGGCCCACTTGGCAGTACATCAATATCTATTAATAGTAACTTGGCAAGTACATTACTATTGGAAGGACGCCAGGGTACATTGGCAGTACTCCCATT GACGT 


\section{Appendix B}

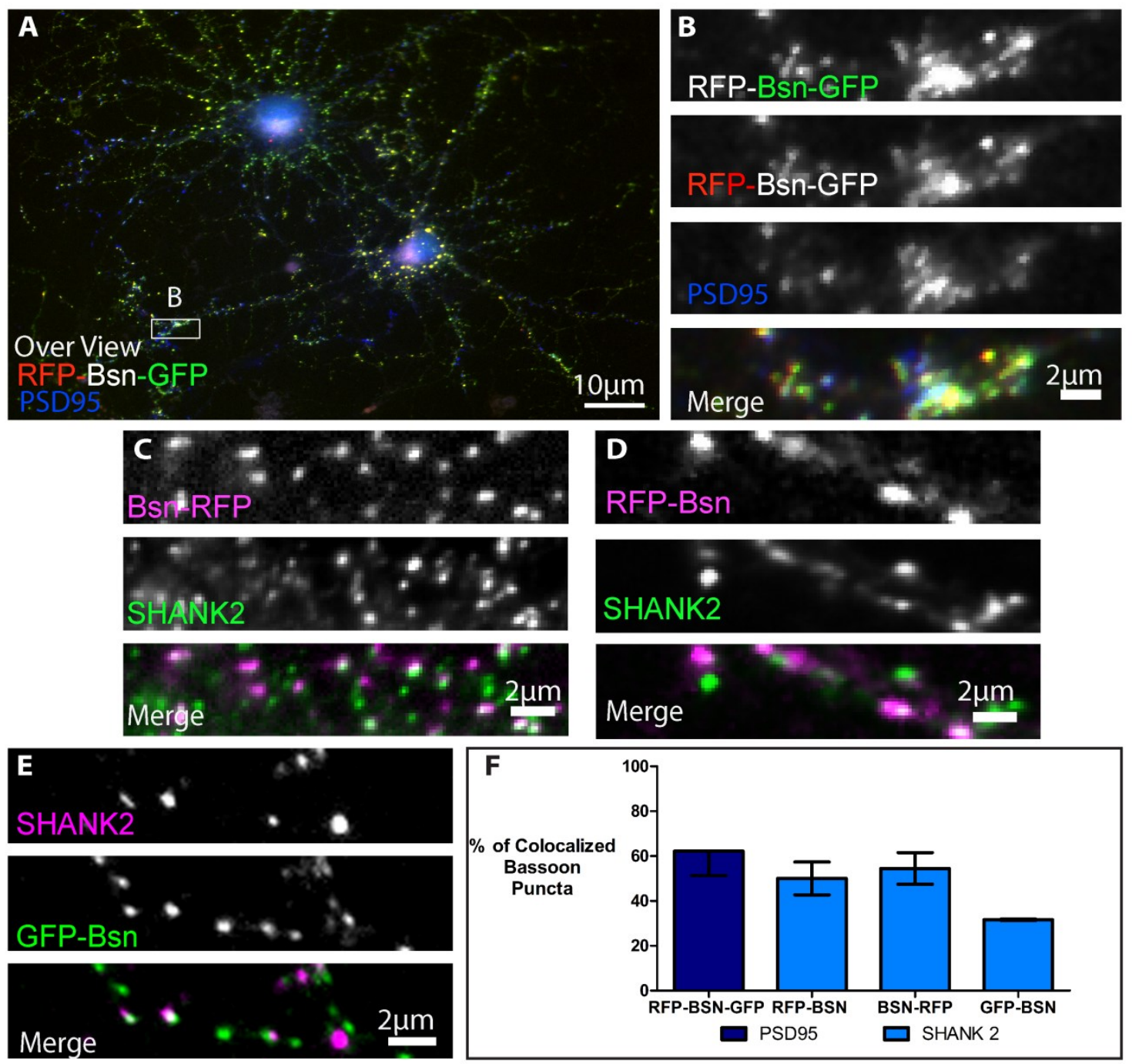

Appendix B. Colocalization of Full-length Bassoon constructs to post-synaptic densities. A, B double-tagged Bassoon construct (over view and zoom images) and C, D and $\mathbf{E}$ zoom images of single-tagged Bassoon constructs in the processes of transfected DIV14 hippocampal neurons, co-immunostained with SHANK2 (post-synaptic marker). Graph F quantification of the colocalized Bassoon puncta populations. $\mathrm{N}=4$ cells from 2 sets. Scale bars: $\mathbf{A}-10 \mu \mathrm{m}$ and $\mathrm{B}-\mathrm{E}-2 \mu \mathrm{m}$. 


\section{Appendix C}

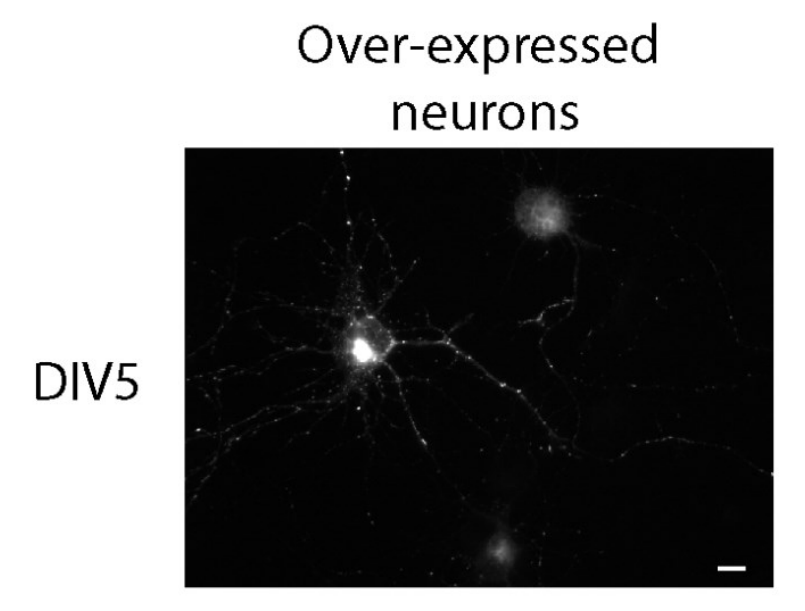

\section{Medium-expressed neurons}
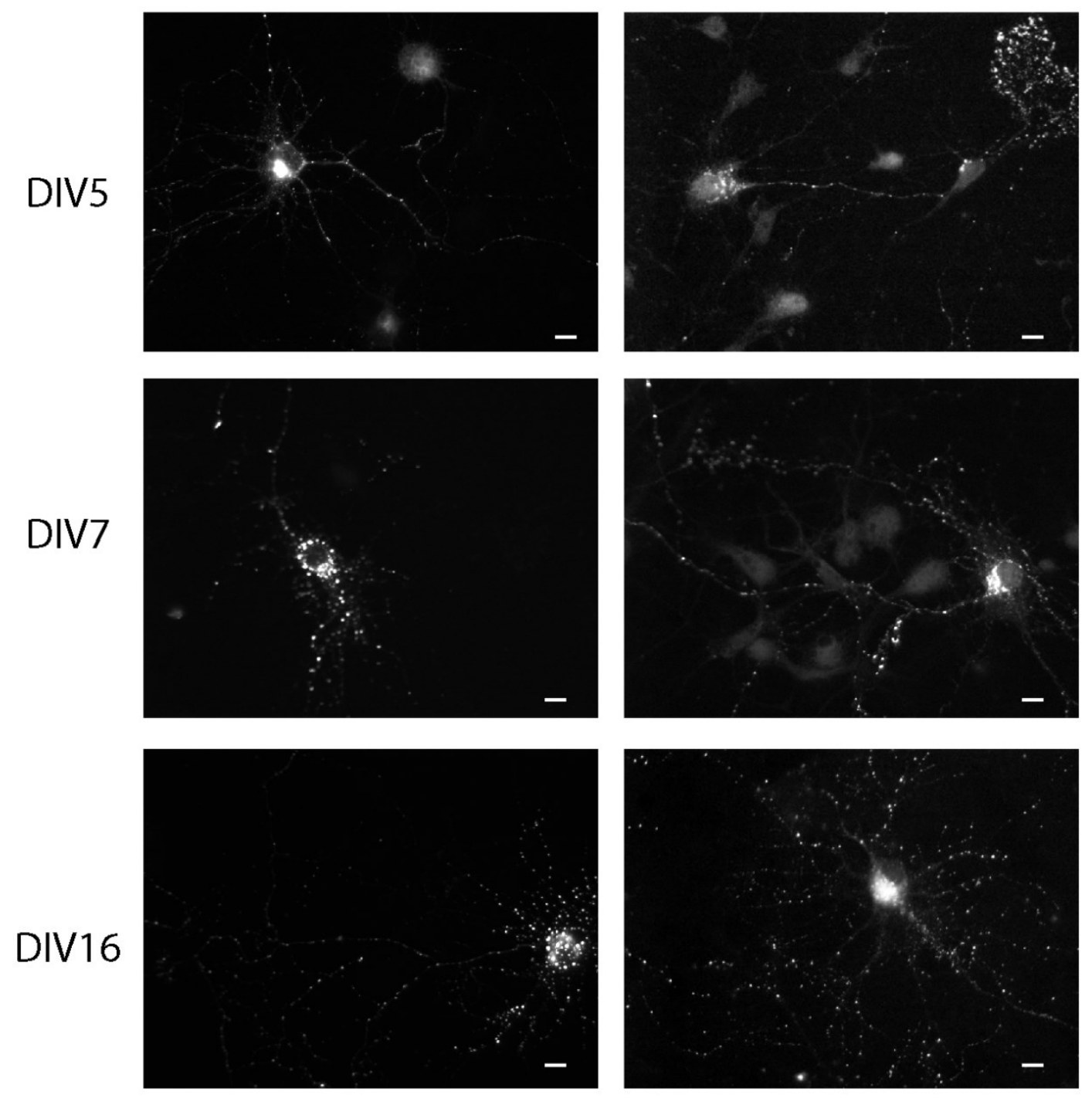

Appendix C. Over-expressed and medium expressed full-length single-tagged Bassoon constructs in young and matured hippocampal neurons. Epifluorescence images of Bassoon transfection in young DIV5-7 neurons and DIV14 matured neurons. Over-expressed neurons have high intensity signals which are bassoon over-expression artifacts, roughly $1 \mu \mathrm{m}$ large clusters, present in the soma and in the neuronal processes. While medium-transfected neurons have Bassoon signals at juxta-nuclear signals, which are usually seen as Golgi-lamella-likestructures with epifluorescence images. These signals travel out as punctate signals into axons and even decorate large growth cones in young neurons. Matured medium transfected neurons also have, although less intense, Golgi-lamella-like-signals in the soma and their punctate bassoon signals are seen in neighboring neuronal processes. Scale bar: 10 $\mu \mathrm{m}$. 


\section{Appendix D}
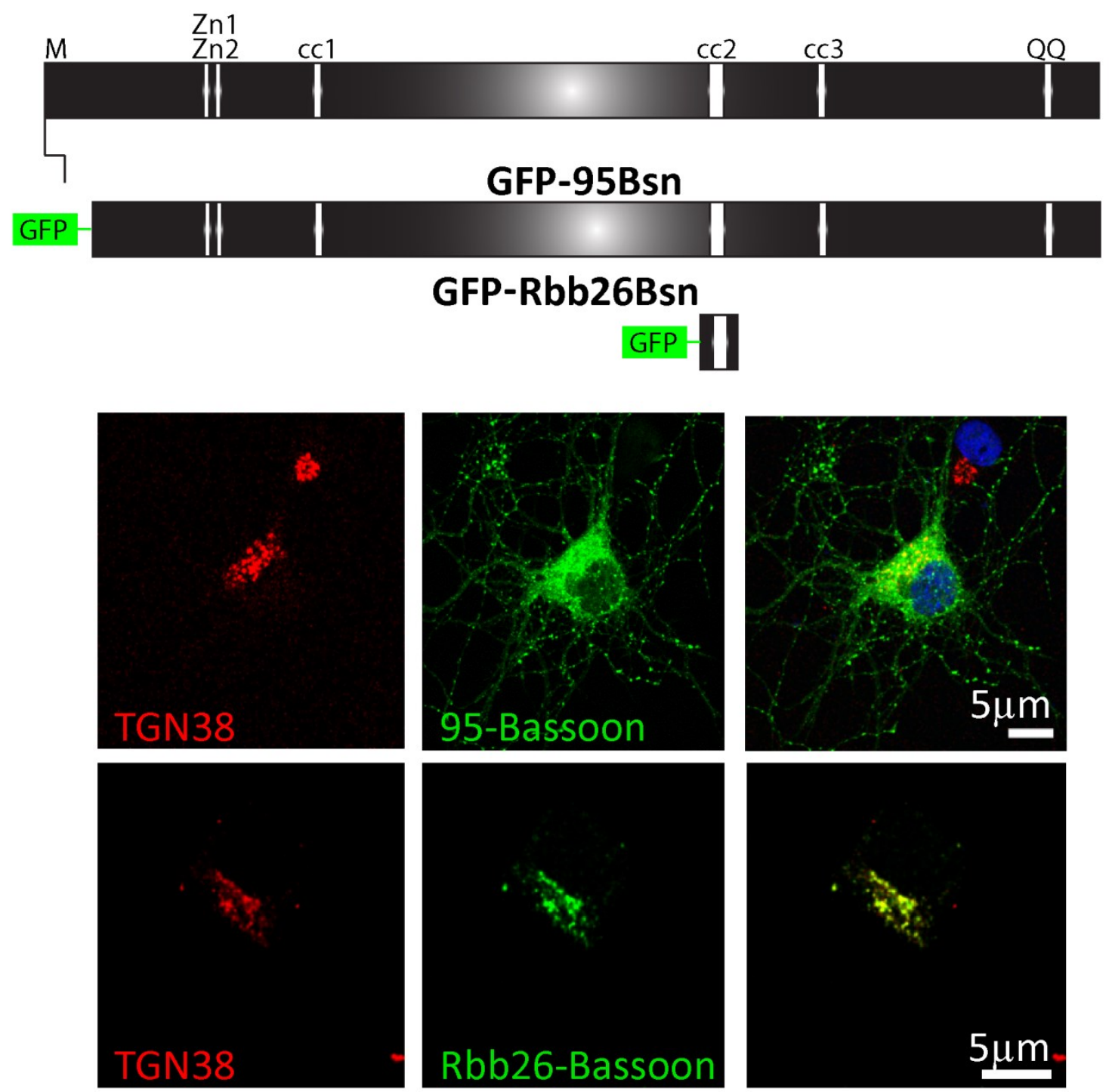

Appendix D. Localization of Rbb26-Bsn and 95-Bsn construct at the TGN38 lamella. A construct sequence map on top shows the sized of the Bassoon molecules transfected. DIV6 Lipofectamine transfected hippocampal neurons fixed with PFA at DIV7. Confocal were acquired of the neuronal soma and TGN38 antibody staining and GFP autofluorescence is visualized. Scale bar: $5 \mu \mathrm{m}$. 


\section{Appendix E}
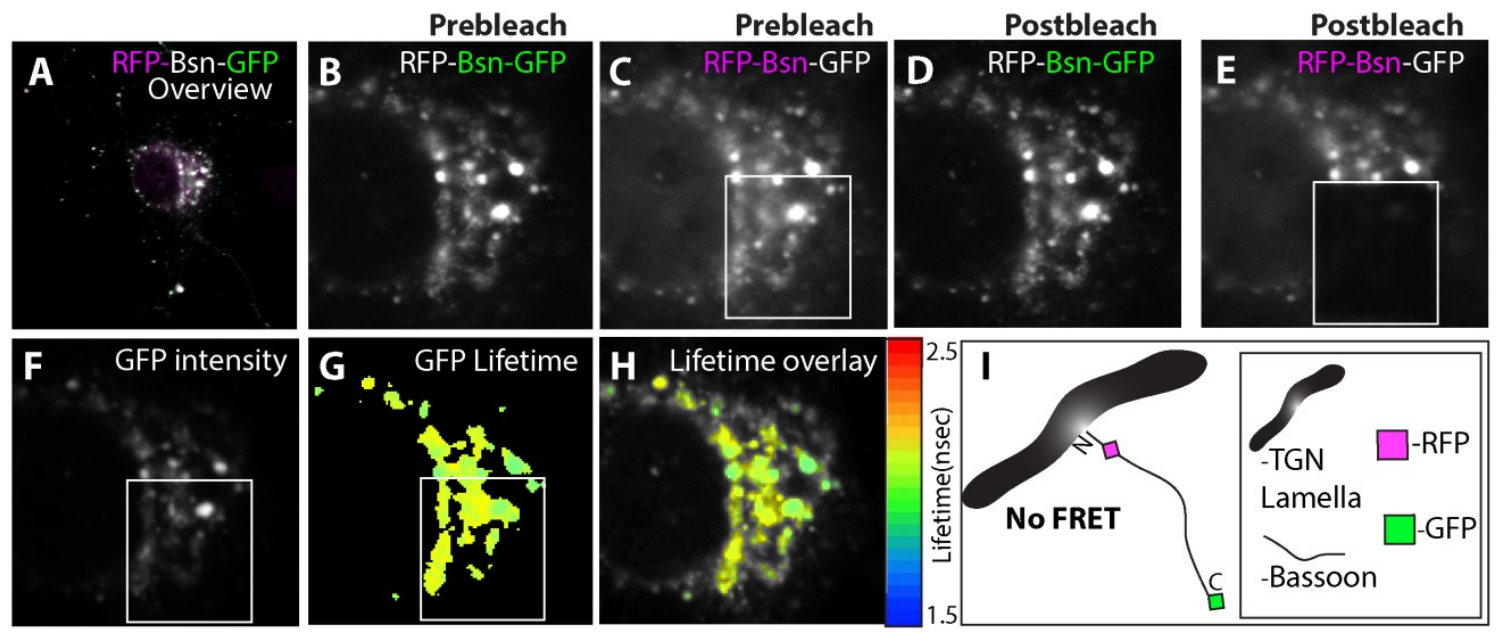

Appendix E: Double-tagged full-length Bassoon never orients in anti-parallel confirmation and a molecule's $\mathrm{N}$ - and C-termini are not localized within $5 \mathrm{~nm}$ of each other in the soma. A, Transfected DIV7 hippocampal neuronal soma, B-E represents fluorescence images of A, preand post- acceptor bleaching in the outlined ROI. F-H represented the lifetime information obtained and overlaid. No warmer LUT ranges in the bleached ROI was observed, therefore none of the signals in the rest of the image is indicated to possess lower FRET lifetimes. I, represents a schematic of Bassoon's conformation at the TGN. 


\section{Appendix F}

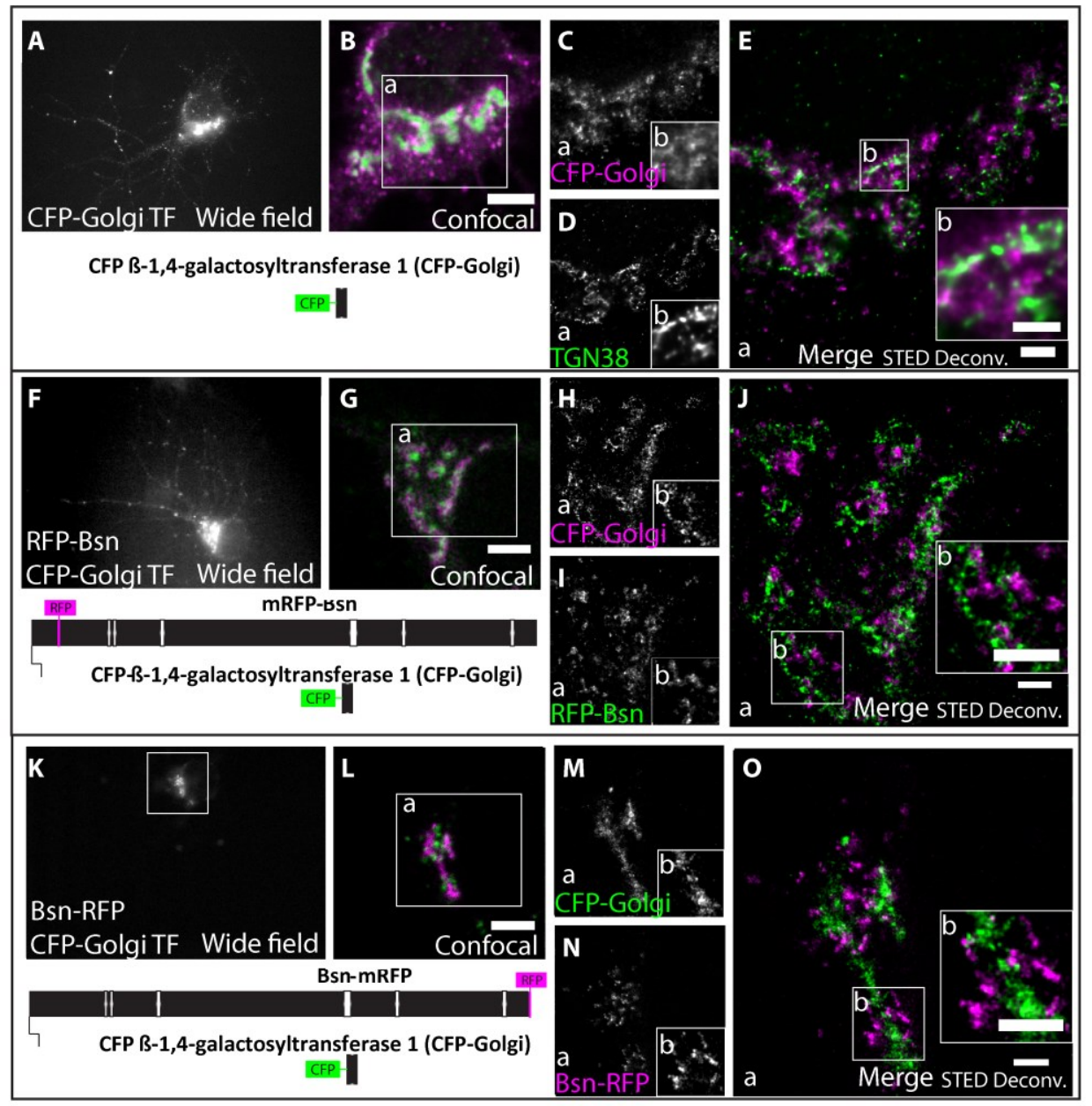

Appendix $\mathrm{F}$ : $\mathrm{N}$ and $\mathrm{C}$ terminus of full-length Bassoon localizes at the trans-Golgi subcompartment. DIV7 hippocampal neurons were transfected with CFP-Golgi (trans-Golgi subcompartment maker), full-length single-tagged mRFP-Bsn or Bsn-mRFP constructs, and immunostained using GFP and/or RFP nanobodies against tagged constructs and from A-E with TGN38 (trans-Golgi network maker). Two-color STED images of both Golgi sub-compartment markers (A-E), CFP-Golgi and RFP-Bsn constructs (F-J), and CFP-Golgi and RFP-Bsn (KO). A, F, K show wide field over view of transfected construct, $\mathbf{B}, \mathbf{G}, \mathbf{L}$ the confocal zoomed image of the soma, inset a reflects both the single channels and merged STED deconvolved (Deconv.) images of $\mathbf{C}-\mathbf{E}, \mathbf{H}-\mathbf{J}$, and $\mathbf{M}-\mathbf{O}$, and $\mathbf{b}$ the STED zooms. With the images in each panel, a schematic diagram of the transfected constructs used is supplied. Scale bars $4 \mu \mathrm{m}$ (B, G and $\mathbf{L}$ ) and $1 \mu \mathrm{m}(\mathbf{E}, \mathbf{J}$ and $\mathbf{O})$. 


\section{Appendix G}

\begin{tabular}{|c|c|c|}
\hline & $0-100 \mathrm{~nm}$ & $101-1000 \mathrm{~nm}$ \\
\hline [N] RFP-Bsn-GFP & $\begin{array}{c}32.5 \mathrm{~nm} \pm 8.0 \mathrm{~nm} \\
(67 \% \text { of the population) }\end{array}$ & $\begin{array}{c}210.6 \mathrm{~nm} \pm 13.0 \mathrm{~nm} \\
\text { (33\% of the population) }\end{array}$ \\
\hline RFP-Bsn-GFP[C] & $\begin{array}{c}60.3 \mathrm{~nm} \pm 6.8 \mathrm{~nm} \\
\text { (37\% of the population) }\end{array}$ & $\begin{array}{c}229.4 \mathrm{~nm} \pm 68.4 \mathrm{~nm} \\
\text { (63\% of the population) }\end{array}$ \\
\hline RFP-Bsn & $\begin{array}{c}30.5 \mathrm{~nm} \pm 6.9 \mathrm{~nm} \\
\text { (84\% of the population) }\end{array}$ & $\begin{array}{c}154.1 \mathrm{~nm} \pm 12.01 \mathrm{~nm} \\
\text { (16\% of the population) }\end{array}$ \\
\hline Bsn-RFP & $\begin{array}{c}50.3 \mathrm{~nm} \pm 3.03 \mathrm{~nm} \\
\text { (46\% of the population) }\end{array}$ & $\begin{array}{c}192.1 \mathrm{~nm} \pm 11.58 \mathrm{~nm} \\
\text { (54\% of the population) }\end{array}$ \\
\hline GFP-95Bsn & $\begin{array}{c}51.3 \mathrm{~nm} \pm 12.4 \mathrm{~nm} \\
\text { (40\% of the population) }\end{array}$ & $\begin{array}{c}231.9 \mathrm{~nm} \pm 41.0 \mathrm{~nm} \\
\text { (60\% of the population) }\end{array}$ \\
\hline GFP-Rbb26Bsn & $\begin{array}{c}52.2 \mathrm{~nm} \pm 12.2 \mathrm{~nm} \\
\text { (33\% of the population) }\end{array}$ & $\begin{array}{c}243.0 \mathrm{~nm} \pm 29.06 \mathrm{~nm} \\
\text { (67\% of the population) }\end{array}$ \\
\hline
\end{tabular}

Appendix G: Table 6. Average distances of full-length and deletion mutants of Bassoon to the closest TGN38 signal. These are the accumulated values in the two population distributions of $0-100 \mathrm{~nm}$; representing signals close to the TGN and $101-1000 \mathrm{~nm}$; representing signals further away from the TGN. The green highlighted panels represent the dominant distance localization of all the Bassoon constructs. 


\section{Acknowledgements}

I would first like to thank Dr. Nina Wittenmayer for picking me out of several applicants for this position and providing me with continuous supervision and mentoring during the entire course of my PhD project. Thank you for showing me how to run and organize my projects and collaborations and for constantly supporting my work style and independence. I would like to thank Prof. Thomas Dresbach for entrusting me to characterize and use the new full-length Bassoon constructs and take my project to exciting avenues. I am extremely grateful for your constant support and supervision, the many long hours of discussion and wisdom that I have so freely received time and again, and for constantly making me feel like a valued member of your team.

A big part of my success has unequivocally been a result of the experiences that I have gained from Göttingen's science community, the wealth of mentors, collaborators, and fellows from the GGNB-CMPB PhD school and the Dorothea Schlözer Mentoring program.

I would like to thank Prof. Micheal Hörner for always believing in my potential, for providing me with support and guidance on every topic concerning my PhD process. I am especially grateful to you for making me a part of a PhD school that provided me with so many opportunities to network, to travel to further educate myself, to present my work at local and international conferences and armed me with a formidable network of friends.

I would like to thank my thesis committee members Prof. Blanche Schwappach and Prof. Fred Wouters. I have always been extremely grateful for your unbiased and critical comments, which have helped me steer my project, and the constant support in the form of personal advice, taking out time from your busy schedules to spontaneously look at my new data and always providing me any type of technical support you could provide from your labs. Your input has been incalculably valuable in shaping not only the content of my work, but they way I question and think about science. I would also like to thank Dr. Camin Dean for not only agreeing to be on my thesis committee panel, but also for providing me your scientific opinion on raw data, so many reagents, and the technical expertise of your lab over the years.

I would like to thank Prof. Mary Osbourne for taking out the time so many times to inspire and illuminate me about a science career and my potential, and Prof. Stefanie Pöggler for mentoring me through my $\mathrm{PhD}$ career.

I have had the unique opportunity during my $\mathrm{PhD}$ life to work as much in my own lab as in my collaborator's labs. I would like to therefore take this opportunity to thank my supervisors for providing the opportunity and all the people in Prof. Stefan Hell's, Prof. Fred Wouter's, and Prof. Anna Fejtova's labs for making me 
feel so welcome to work long, and often inconvenient, hours in your lab. In specific I would like to thank Fabian Göttfert, Rene Ebrecht, Dirk Kamin, and Carolina Montenegro for taking so much of their own time out to provide me with technical support, productive discussions and so much of their patience, as I was trying to learn and understand what they know best.

Absolutely nothing has made my stay and $\mathrm{PhD}$ journey more fun and balanced than the support of my friends, lab mates, and family members.

I'd like to thank my lab mates Asha, Frieda, Julio, Irmgard, Andonia, Jan, Donatus, Shi, Ronja, Moritz, Rebbeca, and Hermes for always taking time out to give me their scientific opinions, helping me out with an experiment or two when I was overworked, and creating a pleasant work environment.

l'd like to thank the students who contributed to my work, some that is in this thesis and some that didn't make it in here, but nevertheless has immensely helped me get my academic career to where it is now. Thank you Donatus in specific for panel A-E in Figure 10, Ronja and Shi for the many many hours of metamorph and imaris anaylsis you spent on characterizing full-length bassoon constructs and sifting through bassoon distance populations.

l'd like to thank all my friends that have made this period of life so much fun. In particular I'd like to thank Astrid, Carla, Oliwia, Daniela, Andrea, Chris, Ahmed, David, Fadi, Josef, Giovanni, Alex(andra), Anne, Loppo, Thomas, Verena, Clemens, Nora, and Cosi.

For always believing in me, supporting me through my $\mathrm{PhD}$, the many pep talks, back rubs, and editing sessions, l'd like to thank Luke. I couldn't have done this without you!

Lastly and most importantly l'd like to thank all my old-time friends spread all over the world, my parents Harshad and Chetna, and my brothers Chirag and Dhruv, who have at times knowingly, other times unknowingly pushed me to never stop excelling and have been hearing me say that I am going to get a $\mathrm{PhD}$ for the past 15 years. 


\section{Curriculum Vitae}

Tina Ghelani

Date of birth: 02.01.1986

Place of birth: Kolkata, India

\section{EDUCATION}

\begin{tabular}{|c|c|}
\hline $2012-2016$ & $\begin{array}{c}\text { Doctoral studies at the University of Göttingen, Germany } \\
\text { within the GGNB-CMPB PhD school, } \\
\text { Advisors: Prof. Dr. Thomas Dresbach and Dr. Nina } \\
\text { Wittenmayer, Department of Anatomy and Embrology, UMG } \\
\text { Göttingen } \\
\text { Thesis title: }\end{array}$ \\
"Orientation and organization of the presynaptic \\
active zone protein Bassoon: \\
from the Golgi to the synapse"
\end{tabular}


Notes

Notes 
Notes 